LUÍS FERNANDO NOGUEIRA DE SÁ

Topology Optimization Method Applied to Ventricular Assist Device Impeller and Volute Design 
LUÍS FERNANDO NOGUEIRA DE SÁ

\title{
Topology Optimization Method Applied to Ventricular Assist Device Impeller and Volute Design
}

\author{
Revised Version
}

Ph. D. Thesis submitted to the Escola Politécnica, University of São Paulo, in partial fulfilment of the requirements for the degree of Doctor of Science.

Concentration area:

Control and Mechanical Automation

Engineering (3152)

Advisor:

Prof. Dr. Emílio Carlos Nelli Silva 
Autorizo a reprodução e divulgação total ou parcial deste trabalho, por qualquer meio convencional ou eletrônico, para fins de estudo e pesquisa, desde que citada a fonte.

Este exemplar foi revisado e corrigido em relação à versão original, sob responsabilidade única do autor e com a anuência de seu orientador.

São Paulo, de de

Assinatura do autor:

Assinatura do orientador:

\section{Catalogação-na-publicação}

Sá, Luís Fernando Nogueira de

Topology Optimization Method Applied to Ventricular Assist Device Impeller and Volute Design / L. F. N. Sá -- versão corr. -- São Paulo, 2019. $152 \mathrm{p}$.

Tese (Doutorado) - Escola Politécnica da Universidade de São Paulo. Departamento de Engenharia Mecatrônica e de Sistemas Mecânicos.

1.Métodos Topológicos (Otimização) 2.Dinâmica dos Fluidos (Simulação) 3.Bombas Centrífugas 4.Bioengenharia I 5. Hemodinâmica I.Universidade de São Paulo. Escola Politécnica. Departamento de Engenharia Mecatrônica e de Sistemas Mecânicos II.t. 
Sá, Luís Fernando Nogueira. Topology Optimization Method Applied to Ventricular Assist Device Impeller and Volute Design. 2019. 153 p. Ph. D. Thesis (Doctor of Science) - Escola Politécnica, University of São Paulo, São Paulo, 2019.

Approved in:

Examination Committee

Prof. Dr.

Institution:

Judgement:

Prof. Dr.

Institution:

Judgement:

Prof. Dr.

Institution:

Judgement:

Prof. Dr.

Institution:

Judgement:

Prof. Dr.

Institution:

Judgement: 
In memory of my grandfather Hélio, who will always be present in my mind at every stage of my life. 


\section{ACKNOWLEDGEMENTS}

I want to thank my supervisor, Professor Dr. Emílio Carlos Nelli Silva, for the attention, discussions, and suggestions given during the execution of this thesis. His guidance and support were precious for the development of this work.

I am grateful to my parents, Claudio N. de Sá and Roseli B. N. de Sá, and my brother, André N. de Sá, not only for the education and formation proportioned but also for the moments of happiness. I thank you all for the unconditional support to all my decisions. Also to Shivinha and Kirinha, for loyalty and caring. Also, in special, to my grandfather, Hélio N. de Sá, for the life lessons and guidance on being a better person.

Exceptional gratitude goes to my girlfriend Jacqueline Kian, for the love, understanding, and patience during the execution of this work.

I am also grateful to my closest friends Igor Meira, Jone Chen, Caio Gutierrez, Natália Salgado, and Amanda Nolasco, for the support and friendship.

With a special mention to my laboratory colleagues Ricardo Amigo, Paulo Yamabe, Ruben Sallas, Diego Prado, Bruno Caldas, Francisco Oliveira, Carlos Okubo, Luís Garcia and André Ferreira for the academic discussions, companionship, and brilliant coffee moments.

I also would like to thank FAPESP (São Paulo Research Foundation) for the financial support to this research under grant 2016/19261-7. 


\section{RESUMO}

Sá, Luís Fernando Nogueira. Topology Optimization Method Applied to Ventricular Assist Device Impeller and Volute Design. 2019. 153 p. Ph. D. Thesis (Doctor of Science) - Escola Politécnica, University of São Paulo, São Paulo, 2019.

Dispositivos de auxílio ventricular (DAV) são essencialmente bombas de pequena escala que auxiliam o bombeamento de sangue. O projeto de DAVs tem como principal requisito baixa tensão de cisalhamento a fim de evitar a destruição de hemácias. As topologias do rotor e voluta dessas bombas influenciam diretamente a condição do escoamento e consequentemente a tensão de cisalhamento. Este trabalho visa o otimizar o rotor e a voluta de um DAV radial, baseando-se no método de otimização topológica. A solução das equações constitutivas é feita utilizando-se o método de elementos finitos para a modelagem do escoamento de sangue, em um referencial rotativo no caso do rotor, e fixo no caso da voluta. O escoamento foi modelado com base no escoamento fluido em meio poroso utilizando a equação de Brinkman; além disso é considerado escoamento turbulento utilizando-se o modelo de Spalart-Allmaras. A função objetivo considerada é a minimização da energia de dissipação viscosa, visando-se, indiretamente, a redução da taxa de hemólise. A implementação dos algoritmos é realizada utilizando-se o ambiente FEniCS em conjunto com as bibliotecas do dolfin-adjoint e pyIpopt. As topologias otimizadas foram construídas utilizando-se impressão 3D. Os protótipos foram testados com água e os resultados utilizados para calibrar um modelo computacional 3D de um DAV completo. Por fim, o modelo foi simulado com sangue operando a $5 \mathrm{l} / \mathrm{min}$ e $100 \mathrm{~mm} \mathrm{Hg}$, e comparado com um rotor de pás retas, observando-se uma redução de $20 \%$ no valor de dissipação viscosa.

Palavras-chave: DAV, Projeto de Rotor, Projeto de Voluta, Otimização Topológica 


\section{ABSTRACT}

Sá, Luís Fernando Nogueira. Topology Optimization Method Applied to Ventricular Assist Device Impeller and Volute Design. 2019. 153 p. Ph. D. Thesis (Doctor of Science) - Escola Politécnica, University of São Paulo, São Paulo, 2019.

Ventricular assist devices (VADs) are mainly small-scale pumps that assist the blood to flow. The design of VAD has as the primary requirement low shear stress in order to avoid the death of red blood cells. The topologies of the rotor and volute of the VAD can directly influence the flow condition. This work aims to optimize the design of the rotor and volute of a radial VAD, based on the topological optimization method. The constitutive equations are solved by using the finite element method for the Carreau-Yasuda blood flow model, in a rotational reference in the case of the rotor, and in a fixed reference frame in the case of the volute. The fluid flow is modeled in porous media using the Brinkman equation; also, the turbulence model of Spalart-Allmaras is used. The objective function is the minimization of viscous energy dissipation, aiming to minimize the hemolysis rate indirectly. The implementation of the algorithms is performed using the FEniCS environment coupled with the dolfin-adjoint and pyIpopt libraries. The optimization problem is solved using the PyIpopt algorithm. The optimized topologies are built using $3 \mathrm{D}$ printing. The prototypes are tested with water, and the results are used to calibrate a 3D complete VAD computational model. Finally, the model is simulated with blood at $5 \mathrm{l} / \mathrm{min}$ and $100 \mathrm{~mm} \mathrm{Hg}$ and compared with a straight blade impeller. A reduction of $20 \%$ in the viscous dissipation is observed.

Key words: VAD, Impeller design, Volute design, Topology Optimization 


\section{LIST OF FIGURES}

Figure 1.1 - Blade splitters . . . . . . . . . . . . . . . . . . . . . . . 21

Figure 1.2 - Three optimization approaches applied to design of a flow channel by considering minimization of pressure loss $\ldots \ldots \ldots \ldots . \ldots 24$

Figure 1.3 - Topology optimization method procedure for impeller design . . . . . 25

Figure 2.1 - Impeller Model approximation by using a Rotational Straight Channel Flow . . . . . . . . . . . . . . . . . . . . . . . . . . 29

Figure 2.2 - Shear rate dependant viscosity for Carreu-Yasuda model (logarithmic scale $\ldots \ldots \ldots \ldots \ldots \ldots \ldots \ldots \ldots \ldots$

Figure 3.1 - Degree of modelling versus Computational Cost $\ldots \ldots$. . . . . . 32

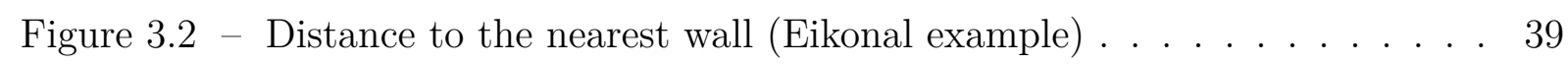

Figure 4.1 - Element Interpolation for Navier-Stokes Eq . . . . . . . . . . . . . . . 41

Figure 4.2 - Element Interpolation for $\nu_{t}$ from the Spalart-Allmaras Eq $\ldots . .442$

Figure 4.3 - Element Interpolation for $\phi$ from the Eikonal Eq . . . . . . . . . . 42

Figure $5.1-\kappa$ as function of parameters $\alpha$ and $q \ldots \ldots \ldots \ldots$

Figure 5.2 - L-shape Example . . . . . . . . . . . . . . . . . . . 47

Figure 5.3 - Horizontal Region Analyze . . . . . . . . . . . . . . . . . . 48

Figure 7.1 - Methods for obtaining the adjoint code . . . . . . . . . . . 58

Figure 7.2 - Topology optimization implementation flow chart . . . . . . . . 60

Figure 7.3 - Iteration Step to solve the forward problem in the turbulent case. . . 61

Figure 8.1 - Impeller domain interpretation $\ldots \ldots \ldots$. . . . . . . . . 62

Figure 8.2 - Impeller mesh . . . . . . . . . . . . . . . . . . . . 62

Figure 8.3 - Boundary conditions for the turbulent rotor optimization with arbitrary dimensions . . . . . . . . . . . . . . . . . 63

Figure 8.4 - Initial guess: Curved Blade . . . . . . . . . . . . . . . . . 64

Figure 8.5 - Optimized topologies for Low Reynolds Case . . . . . . . . . . . . 64

Figure 8.6 - Post-processed topologies for Low Reynolds Case calculated with NS

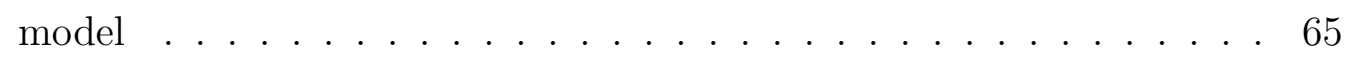

Figure 8.7 - Optimized topologies for High Reynolds Case $\ldots \ldots$. . . . . . 67

Figure 8.8 - Post-processed topologies for High Reynolds Case $\ldots \ldots \ldots$. . . . 68

Figure 8.9 - Boundary conditions for the rotor optimization $\ldots \ldots \ldots$. . . 70

Figure 8.10 - Straight blade and Optimized topologies for $\beta_{g e o}=120^{\circ} \ldots \ldots$. . . 71

Figure $8.11-$ Convergence curves for $\beta_{\text {geo }}=120^{\circ} \ldots \ldots \ldots \ldots \ldots 72$

Figure 8.12 - Velocity field for Straight blade and Optimized topologies $\left(\beta_{\text {geo }}=120^{\circ}\right) 72$

Figure 8.13 - Pressure field for Straight blade and Optimized topologies $\left(\beta_{g e o}=120^{\circ}\right) 73$

Figure 8.14 - Viscosity field for Straight blade and Optimized topologies $\left(\beta_{g e o}=120^{\circ}\right) 73$ 
Figure 9.3 - Picture of the $3 \mathrm{D}$ printed parts $\ldots \ldots \ldots \ldots$

Figure 9.4 - Experimental site scheme . . . . . . . . . . . . . . . . . . . 100

Figure 9.5 - Experimental site photo . . . . . . . . . . . . . . . . . 101

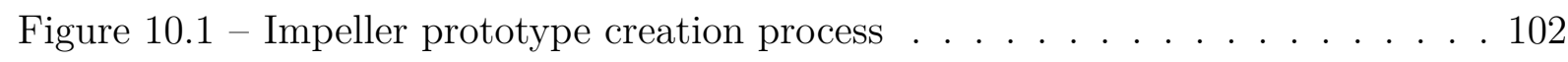

Figure 10.2 - Experimental curve for straight and optimized blade prototypes . . . 103

Figure 10.3 - Simulation Analysis Steps … . . . . . . . . . . . . . . . . 104

Figure 10.4-Boundary Conditions for the ANSYS model (complete pump) . . . . 105

Figure 10.5 - Experimental and Computational Data Comparison . . . . . . . . . 106

Figure 10.6 - Energy Dissipation and Vorticity Comparison between Straight and Optimized Blade Impellers . . . . . . . . . . . . . . . . . . 107

Figure 10.7 - Variable Fields for the optimized impeller ANSYS simulation at 3 $[L / m i n]$ and $3000[\mathrm{rpm}] \ldots \ldots \ldots \ldots \ldots \ldots$

Figure 10.8 - Variable Fields for the straight bladed impeller (SB) ANSYS simulation at $3[\mathrm{~L} / \mathrm{min}]$ and $3000[\mathrm{rpm}] \ldots \ldots$. . . . . . . . . . . . 109

Figure 10.9 - Variable Fields for the optimized impeller ANSYS simulation with blood at $3[L / m i n]$ and $3000[\mathrm{rpm}]] \ldots \ldots \ldots \ldots$. . . . . . . 111

Figure 10.10-Variable Fields for the straight-bladed impeller ANSYS simulation with blood at $3[L / m i n]$ and $3000[\mathrm{rpm}]] \ldots \ldots \ldots . \ldots 112$

Figure 10.11-Variable Fields for the optimized impeller ANSYS simulation with blood at $5[L / m i n]$ and $3400[\mathrm{rpm}] \ldots \ldots \ldots \ldots \ldots . \ldots \ldots$

Figure 10.12-Variable Fields for the straight-bladed impeller ANSYS simulation with blood at $5[L / m i n]$ and $3200[\mathrm{rpm}] \ldots \ldots \ldots . \ldots 115$

Figure B.1 - Boundary conditions for the verification of the implementation. . . . 136

Figure B. 2 - Comparison FEniCS minus ANSYS (Newtonian; $\omega=0[\mathrm{rpm}]$ )] . . . . 137

Figure B.3 - Comparison FEniCS minus ANSYS (Newtonian; $\omega=100[\mathrm{rpm}])]$

Figure B.4 - Comparison FEniCS minus ANSYS (Non-Newtonian; $\omega=0[\mathrm{rpm}])$ ] . 139

Figure B.5 - Comparison FEniCS minus ANSYS (Non-Newtonian; $\omega=100[\mathrm{rpm}])] .140$

Figure B.6 - Comparison FEniCS minus ANSYS (Turbulent Newtonian; $\omega=0[\mathrm{rpm}]$ ) 141

Figure B.7 - Comparison FEniCS minus ANSYS (Turbulent Newtonian; $\omega=$

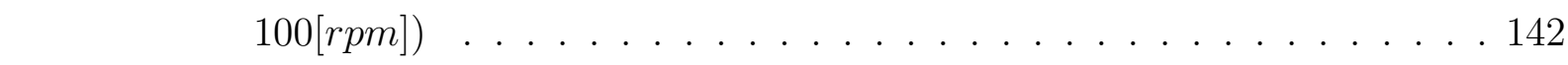

Figure B.8 - Comparison FEniCS minus OpenFOAM (Turbulent Newtonian; $\omega=$

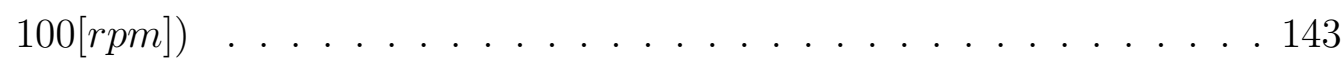

Figure C.1 - Trial points for sensitivity verification . . . . . . . . . . . . . 145

Figure D.1 $-Y+$ distribution for the Straight Blade Case . . . . . . . . . . . . 148

Figure D. $2-Y+$ distribution for the Optimized Blade Case . . . . . . . . . . . . 149

Figure D.3 - Mesh Convergence Curves for Water . . . . . . . . . . . . . . . 150

Figure D.4 - Mesh Convergence Curves for Blood. . . . . . . . . . . . . . . . 152 


\section{LIST OF TABLES}

Table 8.1 - Inputs for Low Reynolds Turbulent Optimization . . . . . . . . . . . . 64

Table 8.2 - Objective function values for Low Reynolds Case calculated with NS model . . . . . . . . . . . . . . . . . . 6 65

Table 8.3 - Objective function values for Post-processed Low Reynolds Case . . 66

Table 8.4 - Inputs for High Reynolds Turbulent Optimization . . . . . . . . . . 66

Table 8.5 - Objective function values for High Reynolds Case calculated with

RANS-SA eqs. . . . . . . . . . . . . . . . . 67

Table 8.6 - Objective function values for Post-processed High Reynolds Case calculated with RANS-SA model . . . . . . . . . . . . . . . . 68

Table 8.7 - Cross-check: Objective function values for Post-processed topologies . 69

Table 8.8 - Inputs for non-Newtonian Impeller Optimization . . . . . . . . . . . 70

Table $8.9-$ Optimization inputs for $\beta_{\text {in }}=120^{\circ} \ldots \ldots \ldots$. . . . . . . . 71

Table 8.10 - Functional values of Straight and Optimized blades with $\beta_{\text {in }}=120^{\circ}$. . 75

Table 8.11 - Functional values of Isolated Straight and Optimized blades with $\beta_{\text {in }}=$

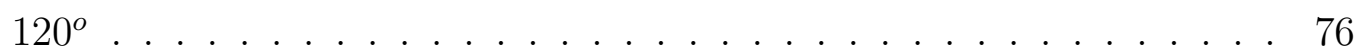

Table 8.12 - Functional values of Straight and Optimized blades with $\beta_{i n}=180^{\circ}$. . 78

Table 8.13 - Functional values of Isolated Straight and Optimized blades with $\beta_{i n}=$

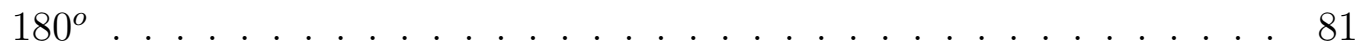

Table 8.14 - Inputs for Turbulent non-Newtonian Impeller forward Problem . . . . 84

Table 8.15 - Optimization Parameters for non-Newt. turbulent impeller optmization 84

Table 8.16 - Functional values for non-Newtonian turbulent impeller optimization . 89

Table 8.17 - Functional values of Complete and Isolated Optimized non-Newtonian Volute . . . . . . . . . . . . . . . . . . . 99 93

Table 8.18 - Functional values of Complete and Isolated Optimized non-Newtonian turbulent volute . . . . . . . . . . . . . . . . . . . . . . . . 97

Table 10.1 - Functional values Straight-bladed and Optimized impellers operating with blood at $3[\mathrm{~L} / \mathrm{min}]$ and $3000[\mathrm{rpm}]$. . . . . . . . . . . . . . . 113

Table 10.2 - Functional values Straight-bladed and Optimized impellers operating with blood at $5[\mathrm{~L} / \mathrm{min}]$ and $100[\mathrm{mmHg}] \ldots$. . . . . . . . . 117

Table B.1 $-L_{2}$ norm of difference for Newtonian Flow . . . . . . . . . . . . . . . 137

Table B.2 $-L_{2}$ norm of difference for Newtonian Flow . . . . . . . . . . . . . . . . 139

Table B.3 $-L_{2}$ norm of difference for Turbulent Flow (FEniCS - ANSYS) . . . . . 142

Table B.4 $-L_{2}$ norm of difference for Turbulent Flow (FEniCS - OpenFOAM). . . 143

Table B.5 - Problem Implementation Comparison . . . . . . . . . . . . . . . . . . 144

Table C.1 - Sensitivity Verification for Newtonian Flow $\left(h=1 \cdot 10^{-6}\right)$. . . . . . . 146

Table C.2 - Sensitivity Verification for Non-Newtonian Flow $\left(h=1 \cdot 10^{-6}\right)$. . . . . 147 
Table C.3 - Sensitivity Verification for Turbulent Flow $\left(h=1 \cdot 10^{-6}\right)$. . . . . . . . 147 Table D.1 - Mesh Convergence Data for the Straight Blade Case with Water . . . 151 Table D.2 - Mesh Convergence Data for the Optimized Blade Case with Water . . 151 Table D.3 - Mesh Convergence Data for the Straight Blade Case with Blood. . . . 153 Table D.4 - Mesh Convergence Data for the Optimized Blade Case with Blood . . 153 


\section{ACRONYMS}

\begin{tabular}{|c|c|}
\hline CAD & Computer Aided Design \\
\hline CFD & Computational Fluid Dynamics \\
\hline CVD & Cardiovascular diseases \\
\hline CY & Carreau-Yasuda \\
\hline FC & Form Compiler \\
\hline FDM & Finite Difference Method \\
\hline FE & Finite Element \\
\hline FEM & Finite Element Method \\
\hline FFC & FEniCS Form Compiler \\
\hline FVM & Finite Volume Method \\
\hline IPOpt & Internal Point Optimizer \\
\hline MUMPS & MUltifrontal Massively Parallel Sparse \\
\hline NS & Navier-Stokes \\
\hline PETSc & Portable, Extensible Toolkit for Scientific Computation \\
\hline PDE & Partial Differential Equation \\
\hline RANS & Reynolds Averaged Navier-Stokes \\
\hline RANS-SA & RANS with Spalart-Allmaras turbulence model \\
\hline $\mathrm{RC}$ & Rotation/Curvature Correction \\
\hline S-A & Spalart-Allmaras \\
\hline SB & Straight Blade \\
\hline SB60 & Straight Blade rotated by 60 degrees \\
\hline SSS & Scalar Shear Stress \\
\hline TAH & Total artificial heart \\
\hline TO & Topology Optimization \\
\hline TOM & Topology Optimization \\
\hline UFC & Unified Form-Assembly Code \\
\hline UFL & Unified Form Language \\
\hline VAD & Ventricular assist device \\
\hline WHO & World Health Organization \\
\hline WSS & Wall Shear Stress \\
\hline
\end{tabular}




\section{NOTATION}

\begin{tabular}{ll}
$\mathbf{u}$ & Velocity field \\
$\mathbf{u}_{a}$ & Absolute velocity \\
$\mathbf{u}_{r}$ & Relative velocity \\
$\boldsymbol{\omega}$ & Angular velocity \\
$\mathbf{r}$ & Radius \\
$p$ & Pressure field \\
$\mathbf{n}$ & Outward-pointing normal \\
$\mu$ & Fluid molecular viscosity \\
$\mu(\dot{\gamma})$ & non-Newtonian viscosity \\
$\mu_{T}$ & Turbulent viscosity \\
$\nu_{t}$ & Spalart-Allmaras eddy viscosity \\
$\phi$ & Wall distance \\
$\alpha$ & Design variable \\
$\epsilon(\mathbf{u})$ & Velocity shear rate \\
$\dot{\gamma}$ & Shear rate \\
$E$ & Energy dissipation (Newtonian laminar) \\
$E_{v i s c}$ & Viscous dissipation \\
$E_{f r i c}$ & Friction dissipation \\
$E_{n n}$ & Energy dissipation (non-Newtonian laminar) \\
$E_{T}$ & Energy dissipation (Newtonian turbulent) \\
$E_{n n_{T}}$ & Energy dissipation (non-Newtonian turbulent) \\
$J_{s s}$ & Scalar Shear Stress \\
$J(\mathbf{u})$ & Vorticity \\
$R_{m}$ & NS Momentum Eq. weak form residual \\
$R_{c}$ & NS Continuity Eq. weak form residual \\
$R_{R A N S m}$ & RANS Momentum Eq. weak form residual (Newtonian fluid) \\
$R_{R A N S c}$ & RANS Continuity Eq. weak form residual \\
$R_{S A}$ & Spalart-Allmaras Eq. weak form residual \\
$R_{e i k}$ & Eikonal Eq. weak form residual \\
$R_{n n_{R A N S m}}$ & RANS Momentum Eq. weak form residual (non-Newtonian fluid) \\
$\mathbf{u}_{\text {wall }}$ & Reynolds number \\
& Inlet velocity \\
\hline${ }_{n}$ & Wall velocity \\
\hline & \\
\hline
\end{tabular}




\section{CONTENTS}

$1 \quad$ INTRODUCTION $\ldots \ldots \ldots \ldots \ldots \ldots \ldots \ldots$

$1.1 \quad$ Ventricular Assist Devices . . . . . . . . . . . . . . . . . . . 18

$1.1 .1 \quad$ Flow Machines . . . . . . . . . . . . . . . . . . . . . . . . . . . 19

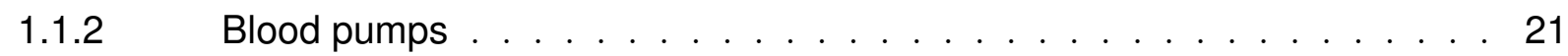

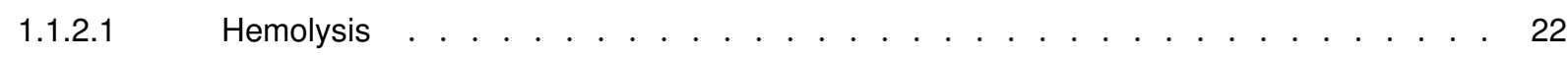

1.1 .2 .2 Thrombosis . . . . . . . . . . . . . . . . . . . . 22

$1.2 \quad$ Optimization Methods $\ldots \ldots \ldots \ldots \ldots$

$1.3 \quad$ Topology Optimization Design Procedure . . . . . . . . . . . 24

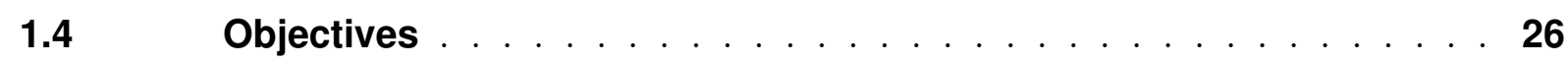

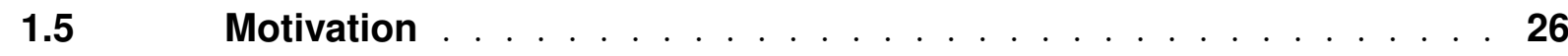

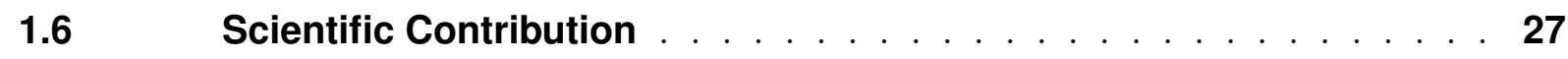

1.7 Document Outline $\ldots \ldots \ldots \ldots$

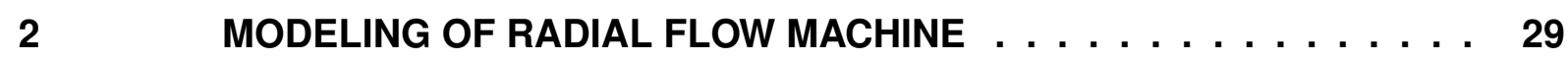

$2.1 \quad$ Equilibrium Equations $\ldots \ldots \ldots \ldots$

$2.2 \quad$ Non-Newtonian Effects $\ldots \ldots \ldots \ldots$

3 TURBULENCE MODEL $\ldots \ldots \ldots \ldots \ldots \ldots \ldots \ldots$

$3.1 \quad$ Reynolds Averaged Navier-Stokes equations (RANS) $\ldots \ldots \ldots$

$3.2 \quad$ Spalart-Allmaras turbulence model . . . . . . . . . . . . . . 35

$3.2 .1 \quad$ Baseline Model . . . . . . . . . . . . . . . . . . . . . . . 35

3.2.2 Preventing Negative Values of Modified Vorticity $\hat{S} \ldots \ldots \ldots$. . . . . 37

$3.2 .3 \quad$ Rotation/Curvature Correction $(\mathrm{RC}) \ldots \ldots \ldots . \ldots . \ldots 37$

$3.3 \quad$ Eikonal Equation $\ldots \ldots \ldots \ldots \ldots$

$4 \quad$ FINITE ELEMENT METHOD APPLIED TO FLOW MACHINES $\ldots \ldots 40$

$4.1 \quad$ Rotational Navier-Stokes Weak Formulation . . . . . . . . . . . . 40

4.2 Rotational RANS Weak Formulation . . . . . . . . . . . . . 41

$4.3 \quad$ Spalart-Allmaras Weak Formulation $\ldots \ldots \ldots . \ldots 42$

$4.4 \quad$ Eikonal Weak Formulation . . . . . . . . . . . . . . . . . . 42

5 TOPOLOGY OPTIMIZATION METHOD . . . . . . . . . . 43

$5.1 \quad$ Topology Optimization Method applied to Fluid Flow Problems . . . . 43

$5.2 \quad$ Topology Optimization Model . . . . . . . . . . . . . . . . 44

$5.2 .1 \quad$ Material Model . . . . . . . . . . . . . . . . . . . . . . . 44

$5.2 .2 \quad$ Non-Newtonian Equation with Material Model $\ldots \ldots \ldots$. . . . . . 46 
$5.2 .3 \quad$ Spalart-Allmaras Equation with Material Model . . . . . . . . . . . . . 46

$5.2 .4 \quad$ Eikonal Equation with Material Model $\ldots \ldots \ldots$. . . . . . . . . . . 46

$5.3 \quad$ Topology Optimization Problem . . . . . . . . . . . . . . 48

$5.3 .1 \quad$ Energy Dissipation . . . . . . . . . . . . . . . . . . . . . 49

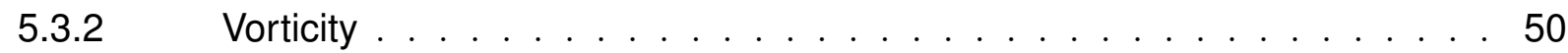

$5.3 .3 \quad$ Non-Newtonian TO Problem Formulation . . . . . . . . . . . . . . . . 51

$5.3 .4 \quad$ Turbulent TO Problem Formulation . . . . . . . . . . . . . 52

$5.3 .5 \quad$ Non-Newtonian Turbulent TO Problem Formulation . . . . . . . . . . . 52

$6 \quad$ SENSITIVITY OF THE TOPOLOGY OPTIMIZATION PROBLEM $\ldots .53$

$6.1 \quad$ Adjoint Method . . . . . . . . . . . . . . . . . 53

$6.1 .1 \quad$ Continuous Adjoint Approach $\ldots \ldots \ldots \ldots$

7 NUMERICAL IMPLEMENTATION $\ldots \ldots \ldots \ldots \ldots \ldots$

$7.1 \quad$ Optimization Algorithm $\ldots \ldots \ldots \ldots \ldots$

7.2 Software Environment . . . . . . . . . . . . . . . . . . 56

$7.2 .1 \quad$ Solving Differential Equations - The FEniCS system . . . . . . . . . 57

$7.2 .2 \quad$ Solvers and Preconditioners . . . . . . . . . . . . . . 57

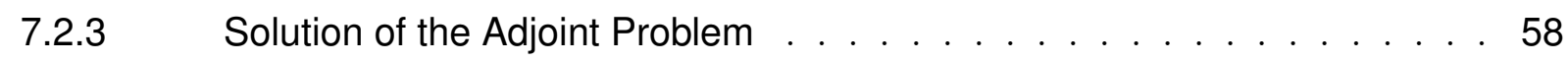

$7.2 .4 \quad$ Solution of the Optimization Problem . . . . . . . . . . . . . . . . 59

$7.2 .5 \quad$ Implementation of Flow Machine Optimization $\ldots \ldots \ldots$. . . . . 60

8 NUMERICAL RESULTS $\ldots \ldots \ldots \ldots \ldots \ldots$. . . . . . . 62

$8.1 \quad$ Impeller Design (Turbulent) $\ldots \ldots \ldots 6$

$8.1 .1 \quad$ Domain and Boundary Conditions . . . . . . . . . . . . . 63

$8.1 .2 \quad$ Low Reynolds Optimization . . . . . . . . . . . . . . . 63

$8.1 .3 \quad$ High Reynolds Optimization . . . . . . . . . . . . . . . . . 66

8.1 .4 Cross Check of Results . . . . . . . . . . . . . . . . . . 68

$8.2 \quad$ Impeller Design (Non-Newtonian Model) $\ldots \ldots \ldots$

$8.2 .1 \quad$ Design Domain of 120 degrees $\ldots \ldots \ldots$. . . . . . . . 71

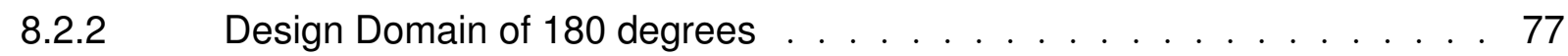

$8.3 \quad$ Impeller Design (Non-Newtonian Turbulent Model) $\ldots \ldots$. . . . . . 83

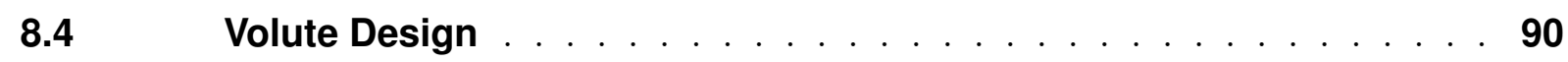

$8.4 .1 \quad$ Volute Design (Non-Newtonian Model) $\ldots \ldots$. . . . . . . . . . 91

$8.4 .2 \quad$ Volute Design (Non-Newtonian Turbulent Model) $\ldots \ldots$. . . . . . . . . 93

9 EXPERIMENTAL METHODOLOGY $\ldots \ldots \ldots \ldots \ldots \ldots$

$9.1 \quad$ Pump Prototype . . . . . . . . . . . . . . . . . . . 98

$9.1 .1 \quad$ 3D Printed Parts . . . . . . . . . . . . . . . . . . . . . . . . 99

$9.2 \quad$ Experimental Setup . . . . . . . . . . . . . . . . . 100

$9.3 \quad$ Experiment Description $\ldots \ldots \ldots \ldots 1$ 
10 EXPERIMENTAL RESULTS $\ldots \ldots \ldots \ldots \ldots$. . . . . . . 102

10.1 Optimized non-Newtonian Turbulent Impeller . . . . . . . . . . . 102

$10.1 .1 \quad$ Experimental Data . . . . . . . . . . . . . . . . . . . . . . 103

$10.1 .2 \quad$ Computational Comparison (Model Calibration) . . . . . . . . . . . 104

$10.1 .3 \quad$ Simulation with Blood $(3[\mathrm{~L} / \mathrm{min}]$ and $3000[\mathrm{rpm}])] \ldots \ldots$

10.1.4 Simulation with Blood $(5[L / m i n]$ and $100[\mathrm{mmHg}])] \ldots \ldots 113$

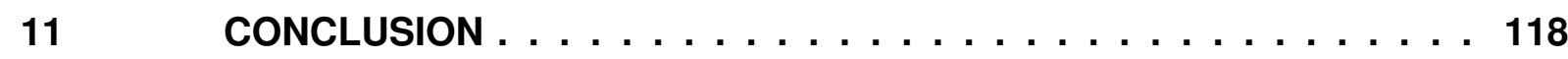

11.1 Future Work $\ldots \ldots \ldots \ldots$

REFERENCES . . . . . . . . . . . . . . . . . 121

APPENDIX A-CONTINUOUS ADJOINT DERIVATION $\ldots \ldots \ldots 133$

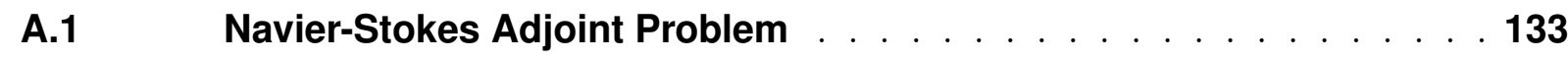

APPENDIX B - VERIFICATION OF THE IMPLEMENTED CODE. . . . 136

B.1 Newtonian Flow . . . . . . . . . . . . . . . . . . . . . . . 137

B.2 Non-Newtonian Flow $\ldots \ldots \ldots$. . . . . . . . . . . . . 138

B.3 Turbulent Flow (S-A) $\ldots \ldots \ldots \ldots \ldots$

APPENDIX C - SENSITIVITY VERIFICATION BY FINITE DIFFERENCE METHOD . . . . . . . . . . . . 145

C.1 Finite Differences $\ldots \ldots \ldots$. . . . . . . . . . . . . . . . . . . . 146

C.2 Newtonian Flow . . . . . . . . . . . . . . . . . . . . . 146

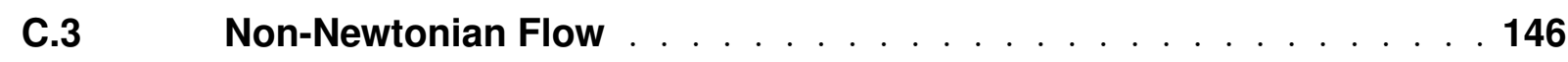

C.4 Turbulent Flow . . . . . . . . . . . . . . . . . . . . . 147

APPENDIX D - MESH CONVERGENCE ANALYSIS . . . . . . . 148

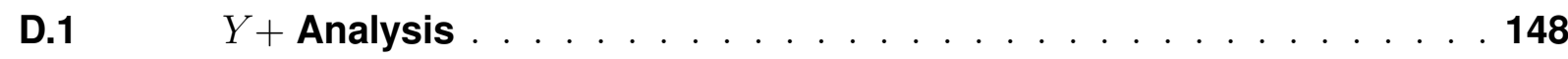

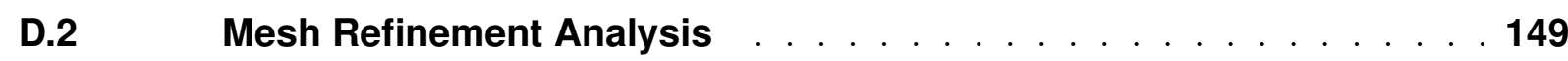




\section{INTRODUCTION}

The World Health Organization (WHO) estimates that 17.7 million people died from cardiovascular diseases (CVD) in 2015, representing 31\% of all global deaths (W.H.O., 2017). Among the CVDs consequences, there is the cardiac insufficiency, which consists in the inability of the heart in maintaining the blood flow to attend metabolic demands (MANN et al., 2015). The cardiac insufficiency thus demands heart transplantation. However, heart transplantation is only attainable by a small group of patients COLVIN-ADAMS et al., 2015). Besides that the time until the patient receives a compatible organ can be very long and thus demands temporary hemodynamic support. The necessity of temporary assistance for patients with heart diseases motivated the creation of different circulatory assist devices, such as the ones analyzed in Kozik and Plunkett (2011).

The circulatory assist devices are, essentially, pumps that assist the blood to flow at adequate rates. They can be used as a temporary solution or as a long term treatment (MCMURRAY et al., 2012). The firsts ventricular assist device (VAD) consisted of a pneumatically driven, tubular displacement pump, and was first reported in 1963 (STEWART; GIVERTZ, 2012). Later, an air-powered balloon pump that inflates the heart chambers providing the circulation by displacing blood was first used clinically in 1968 . Around this time, the idea of a total artificial heart (TAH) implantation emerged and was performed clinically in 1969 (HAN; TRUMBLE, 2019). However, the high fatality rate due to sudden device failures changed the focus to simpler single-chambered mechanical blood pumps, the ventricular assist devices (VADs).

\subsection{Ventricular Assist Devices}

The ventricular assist devices (VADs) can be classified by generation, as described by Gianluca, Francesco and Giuseppe (2013). Pulsatile devices are the first generation of pumps introduced clinically, they have large sizes, multiple moving parts, and can be implanted intra-corporeally or para-corporeally. The second generation are the miniaturized continuous flow pumps that have a single moving part (the impeller). They permit intra-corporeal placement due to their small size. The third generation of blood pumps have non-contact magnetic bearings for the impeller and they have been evaluated in clinical studies more recently.

The VADs are temporary solutions usually being used in cases where the patient is awaiting heart transplantation, working as a bridge between the diagnosis and the surgery (SLAUGHTER et al., 2013). The study of commercial pumps performed by Aaronson et al. 
(2012) shows that these pumps are becoming more and more efficient, and the device can be implanted in patients with advanced levels of heart failure with high 1-year survival (rate), being comparable to heart transplantation.

Kirklin et al. (2010) shows an evaluation of the main aspects involving patients that received ventricular assist devices, indicating that $51.6 \%$ of them use a continuous flow machine and around $87.3 \%$ are patients waiting for transplants or who are going to apply for transplantation. Their study shows the leading death causes in a group of 1092 patients porting the devices and around $4.7 \%$ of the deaths are due to equipment failure, and these failures occur at a rate of 0.1035 failures/patient*year.

The ventricular assist devices are mainly small scale flow machines operating with blood. Thus, a brief introduction to the overall design and optimization of these machines is shown below.

\subsubsection{Flow Machines}

The performance and robustness of these machines are fundamental questions for the industry, wherein small performance improvements can result not only in financial gains for big-scale applications but also in an increase of life expectancy in the case of medical devices. These improvements can be made on all parts of the flow machine, such as the impeller, internal valves, bearings, nozzle, and others. However, the impeller stands out for presenting a large influence on the overall performance. In an experimental work, Yu et al. (2000) estimated losses in the impeller to be about 35\% of total losses. Thus, the impeller design and evaluation of its functioning are important phases of the machine conception process.

The design and performance prediction of the impeller before its construction and implantation are difficult tasks, due to the vast number of variables involved, such as the geometry, position, and number of blades. Therefore, numerical tools are used to obtain an approximation of the characteristics and performance for each impeller design (JAFARZADEH et al., 2011). Thus, the performance enhancement can be obtained by a trial-and-error approach, involving several sequential simulations in which the parameters of the rotor are manually changed at each step. However, this methodology is highly time-consuming and does not imply, necessarily, in performance optimization.

Flow machine optimization comprises everything, from material selection to the better form and position of the blades. In particular, the blade shape optimization has been widely studied. In this process, an initial shape is given, and an algorithm performs local shape changes to improve some characteristics based on the flow around the blade (LEE et al., 2011). This blade shape optimization has, for some specific cases, analytic optimum solutions (HANSEN, 2007). However, for real problems, the boundary conditions that the 
blades are subjected to changes during its life cycle, so the analytic model does not work correctly. Thus, a numerical optimization approach is necessary, and the problem becomes a multi-objective optimization problem without analytic solution (CASAS; PENA; DURO, $2006)$.

A number of works in the literature uses optimization techniques applied to these machines with different approaches, obtaining significant efficiency gains. Among them, the work of Baloni, Pathak and Channiwala (2015) performed a volute optimization based on the Taguchi Method aiming to minimize the losses inside the volute and maximize the stagnation pressure at the volute outlet. They developed a 3-D numerical simulation model using the FLUENT software to simulate a centrifugal air blower. Their results indicate that for an optimum geometry, considering the desired characteristics, the volute width should be 1.5 times the impeller width. With this geometry, higher efficiency is observed, showing an improvement of $7.4 \%$.

Considering the optimization based on the blades, the work of Wen-Guang (2011) developed a 2D blade optimization by using an inverse singularity approach applied to a curved blade design. The results are evaluated by using the CFD code Fluent and showed a $5 \%$ improvement in hydraulic efficiency. Another work involving the blade optimization was performed by Derakhshan et al. (2013), in which a shape optimization based on the Bee Colony algorithm was applied to a centrifugal pump. They started with a curved blade geometry with arbitrary dimensions and then validated the results by using data from the Berkeh 32-160 commercial pump. Their results showed that the optimized shape is very similar to the original shape with small curvature changes and efficiency improvement of $3.59 \%$ was achieved.

There are other design parameters, such as the number of blades, the blade outlet angle, and the impeller outlet diameter, that affect pump performance. In the case of the number of blades, the intuition suggests that a higher number of blades increases the fluid interaction and so it would promote a higher energy transmission. The pump pressure head rises as the number of blades increases. However, the presence of too many blades may cause an increase in the blockage and skin friction in the impeller passage, decreasing efficiency. However, controversially, a method to increase efficiency is the addiction of splitters between the blades. The work of Gölcü, Pancar and Sekmen (2006) shows a study of these splitters. They performed an analysis of impellers with 5, 6 and 7 blades, containing splitters with variable length (from $35 \%$ to $80 \%$ of the blade length) as shown in Figure 1.1. As a result, they achieved an increase in an energy saving of $6.6 \%$ by adding splitters to an impeller with 5 blades and splitters with $80 \%$ of original blade length. 
Figure 1.1 - Blade splitters.

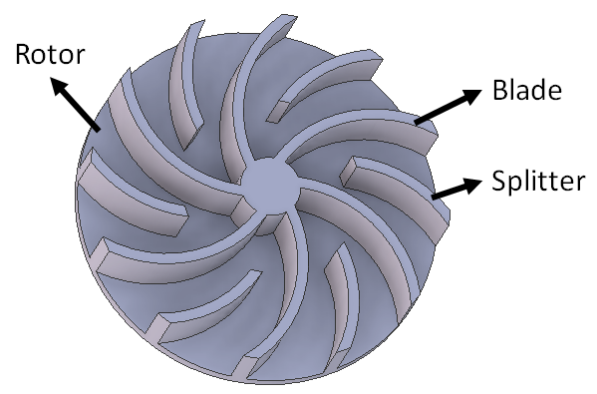

Thus, the optimization of flow machines has been widely studied over the past decades by using different approaches and obtaining meaningful results.

\subsubsection{Blood pumps}

Considering the flow machines operating as blood pumps the main characteristics are no longer only performance related, but the most important aspects are related to the hydraulic efficiency and hematological conditions, which represent the blood cell damage and local coagulation. Thus, the numerical simulations must be capable of offering detailed information about the blood flow inside these machines.

The work of Behbahani et al. (2009) shows a revision of strategies for designing blood pumps based on computational fluid simulations (CFD). A hemocompatibility analysis of a pump is reviewed in the context of hemolysis estimations based on different blood damage models. Also, a rheological model is used to describe the complex blood flow.

The performance improvement for non-newtonian flows (such as blood flows) can be achieved by using optimization methods. The optimization strategy for designing blood pumps has been introduced in Antaki et al. (1995). However, some obstacles made the implementation too difficult. The major challenges are associated, firstly, to the lack of accurate models to describe blood flows, secondly, to a better description of the relations between macroscopic and microscopic characteristics of the blood, and thirdly, to the lack of automatic algorithms to implement the shape changes in functions of the simulation results (ANTAKI et al., 1995). Other works involving the optimization of viscous pumps are Silva, Kobayashi and Coimbra (2007a) and Silva, Kobayashi and Coimbra (2007b).

The criteria for the flow characteristics when designing blood pumps components are (1) to minimize damage to the blood (hemolysis) and (2) to prevent blood clotting (thrombosis). It is well known that damage to the blood is directly related to high shear stress. Furthermore, the existence of stagnation and recirculation regions has shown to have a strong correlation with the onset of coagulation and deposition of blood components within a prosthetic device (ROMERO; N. Silva, 2017). A briefly introduction to hemolysis is shown as follows. 


\subsubsection{Hemolysis}

Hemolysis is the rupture of the red blood cells (erythrocytes) and the release of the hemoglobin in the blood plasma. Hemolysis is an event that occurs not only outside the body, but also inside the body due to some abnormal medical conditions such as bacteria infection, autoimmune disorders or blood with too low solute concentration (BERIS; PICARD, 2015).

Outside the body, when the blood is collected for analysis, hemolysis can occur due to external factors, such as prolonged storage under incorrect conditions and wrongly managing the tubes (MCCAUGHEY et al., 2017). Also, for situations where the blood flows through external devices, such as the VAD case, the hemolysis also occurs due to high rotations, non-biocompatible materials, increased temperature, and increased shear stress (RAVICHANDRAN et al., 2014; LI et al., 2017) among others. Hemolysis is also related to the duration of exposure to a high shear stress (LEVERETT et al., 1972).

Other works have suggested that shear stress has a direct relation with hemolysis rate (ANTAKI et al., 1995, GHATTAS; HE; ANTAKI, 1995). The same publications also indicate that minimizing the overall vorticity is a valid way to optimize the hematological conditions of a blood pump, and is a reasonable choice to reduce recirculation and hence thrombosis. Besides that, the work of Romero and N. Silva (2017) shows that the decrease in vorticity also contributes to the reduction of shear stress functions, thus, diminishing the hemolysis.

\subsubsection{Thrombosis}

Thrombosis is the formation of a blood clot by agglutinating platelets and fibrin inside a blood vessel, obstructing the flow of blood through the circulatory system. This mechanism is a body response to stop blood loss in case of injuries. However, it may occur in other conditions, for example, when the blood enters in contact with external bodies (non biocompatible), which is the case in VADs. The exact mechanism is of thrombo formation is still unclear and some researches indicate that it is a multifactorial phenomenon (URIEL et al., 2014). One of the explanations comes from the theory of Flow-Induced Device Thrombogenicity, in which the flow patterns through the pump resulting in variable turbulence of blood component trajectories are associated with the shear stress and impact thrombus formation (BIRATI; RAME, 2015). Thus, the geometric characteristics of the device may promote thrombus formation (CHIU; SLEPIAN; BLUESTEIN, 2014). In addition, elevated shear stress as well as the generation of heat from the device may contribute to clot formation in critical areas of the pump (SELGRADE; TRUSKEY, 2012).

The shear stress has an important role in the both hemolysis occurrence and thrombus formation and can be predicted by computational simulations (SELGRADE; TRUSKEY, 
2012). Hence, the combination of advanced computational techniques, such as computational fluid dynamics (CFD), and optimization methods can be a powerful approach to design equipment that operates with blood, such as the VADs. The works cited previously show that it is possible to obtain a blood pump with better performance and hematological conditions through optimization methods.

Notice that the focus of this thesis is the methodology to design the impeller and volute of VADs, and more in-depth aspects of the circulatory system can be found in physiological books such as "Ganong's Review of Medical Physiology" (BARRETT et al., 2010) or "Guyton and Hall Textbook of Medical Physiology" (GUYTON; HALL, 2006).

\subsection{Optimization Methods}

The optimization methods are generally divided into three categories: parametric optimization, shape optimization, and topology optimization. Each one of these is better suited for a problem niche, with different constraints and objectives.

Parametric optimization is conceived as a problem where the model is parametrized in a few variables, such as dimensions or rate of dimensions, geometric or material properties. This optimization allows the optimization of both system structure and process parameters. Thus, these variables become the design variables, whose values change as the optimization proceeds until an optimized solution is achieved, considering the problem constraints. The work of Montevecchi et al. (1995) shows an example of the optimization of a blood pump employing the parametric approach.

The shape optimization proceeds with the change of the initial domain shape. The process involves the shape boundary modification to fulfill the imposed constraints adequately. In this method, a shape that serves as a base for the algorithm is initially defined, and then the algorithm modifies the domain contour during the optimization. The resulting geometry has a strong dependence on the initial shape, so the pool of possible solutions is restricted. This dependency can be seen in the work of Derakhshan et al. (2013), where the obtained solution is very similar to the original format. Therefore, this method is better suited to refine previously optimized solutions obtained by other methods.

The third category of optimization, topology optimization, uses a different approach regarding the previous techniques, in the sense that this method involves the material distribution over a specified design domain, which permits the insertion or removal of material at any point of the system, allowing more sophisticated and non-intuitive topologies to be obtained. Hence, the resulting solution does not have a high dependency on the initial configuration, thus, promoting greater freedom for the final topology. Therefore, structures with better performance and more diverse topologies can be reached. 
The three approaches of optimization cited above have already been applied to fluid domains, for example, to obtain flow channels with less pressure loss, as can be seen in Figure 1.2. The first case, illustrating the parametric optimization, shows two parameters defined as design variables, the inlet and outlet diameters. The optimization is performed by selecting the outlet diameter value that minimizes the pressure loss. The second case shows the shape optimization, in which the straight contour is changed to a curved line by changing the control parameters of a spline curve. The third case shows the topology optimization approach to design the optimized geometry, starting with the inlet and outlet boundary conditions and a extended domain and finishing with the material distribution that minimizes the pressure loss.

Figure 1.2 - Three optimization approaches applied to design of a flow channel by considering minimization of pressure loss.

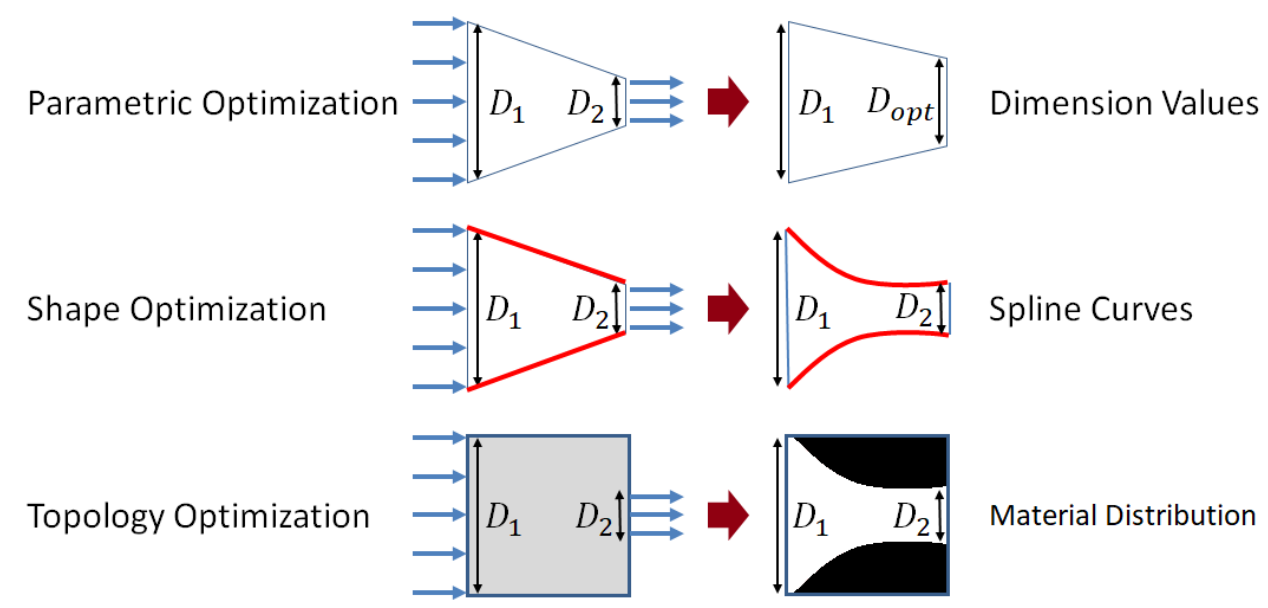

\subsection{Topology Optimization Design Procedure}

The traditional approach to topology optimization consists of a combination of an analysis method, such as the Finite Element Method (FEM), and a numerical optimization algorithm. In the topology optimization method, a design variable is defined over a domain $(\Omega)$, which is modified as the algorithm proceeds.

Initially, a design domain is defined, then a discretization technique is used to produce a mesh, dividing the domain into elements. In principle, any element and mesh geometry can be used to fulfill the project requirements. When the elements are chosen, the nodes and degrees of freedom are defined.

The next step is the definition of the boundary conditions. These are conditions that specify values to the problem variables (Dirichlet Conditions), such as walls with zero velocity, or derivatives of problem variables (Neumann Conditions).

The optimization procedure initiates by defining the objective function based on the desired characteristics. Then, the finite element problem is solved, considering the specified 
mesh and boundary conditions. This gives the velocity and pressure distribution over the domain. Then, the objective function is evaluated, and the gradient of the objective function and constraints with respect to the design variables are calculated. Other constraints, such as the volume constraint, are also verified. The optimization algorithm modifies the design variable following the gradient information, iteratively, to reach an optimized material distribution. The iterative process goes on until the convergence criteria are met.

After the process converges the optimized topology is post-processed, to smooth the contour, and it is re-evaluated to verify whether the constraints are respected. Finally, the design can be built.

Figure 1.3 illustrates this optimization procedure applied to impeller design. The initial domain, the velocity boundary condition at the inlet, and the pressure at the outlet are defined. Then, the domain is discretized, producing the mesh with the desired elements. At the discretization step, the degrees of freedom and variables of the problem are defined (velocity and pressure variables at mesh nodes, for example). The optimized topology is reached after changing the domain topology accordingly to the gradient of the objective function. The final geometry has to be post-processed to smooth its contour. The verification step is necessary to evaluate that the desired objective is reached even with the post-processed modifications. Finally, the optimized geometry can be built.

Figure 1.3 - Topology optimization method procedure for impeller design.

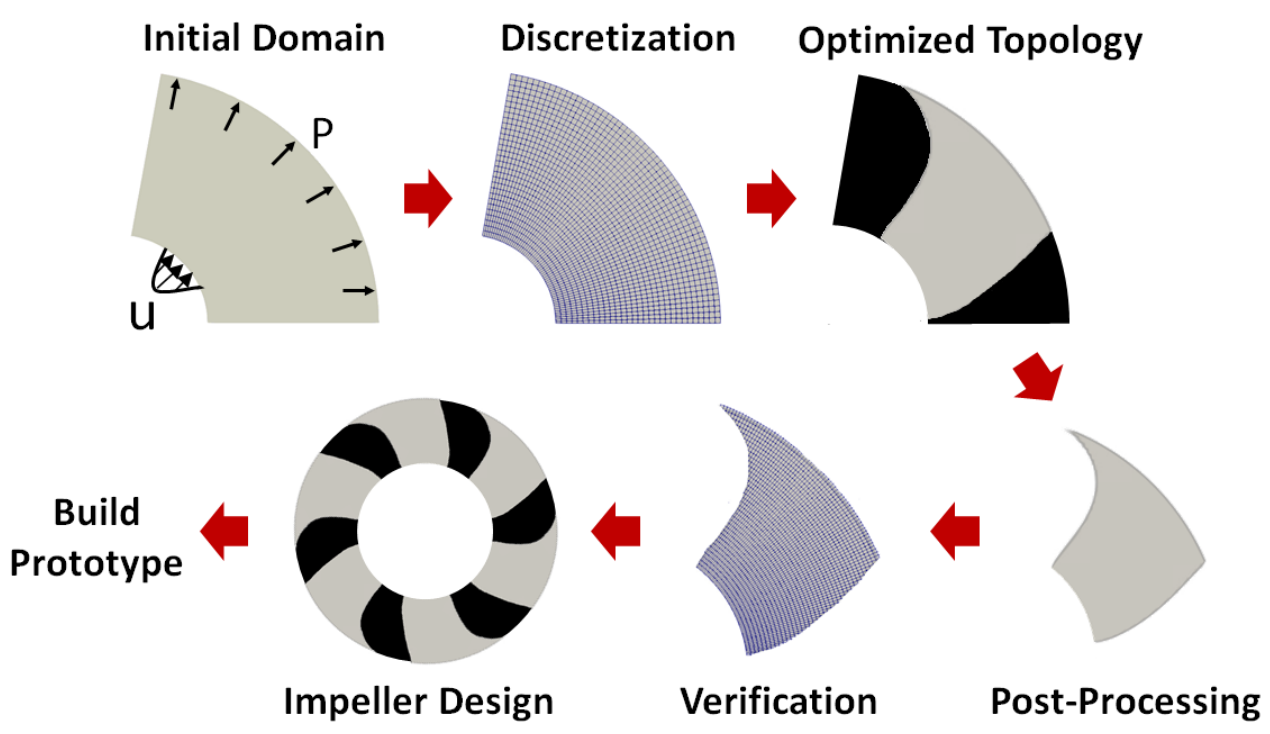




\subsection{Objectives}

This work develops a methodology based on the topology optimization method to the design of the impeller and volute of a radial VAD, which consists of an optimization problem of a radial flow machine considering non-Newtonian fluid and turbulent flow, aiming to optimize not only the pump performance but also the hematological conditions. The model consists of the Reynolds Averaged Navier-Stokes equations combined with the Spalart-Allmaras turbulence model and the fluid viscosity given by the Carreu-Yasuda non-Newtonian model. All state equations are modified to consider the a density based material model. The objective function considered is the energy dissipation, which has shown to be proportional to the shear stress, and to have a high correlation with vorticity.

The state equations are solved by using the finite element method and by implementing the code with the FEniCS libraries. The sensitivities of the optimization problem are calculated via automatic derivation by using the dolfin-adjoint libraries, which uses the adjoint method. The optimizer used is the IPOpt, which offers an implementation of an interior point method.

The scope of this work also considers the complete VAD development, which involves all steps of the design: listing the design requisites, developing simulation models, performing the topology optimization, post-processing, building a prototype, and testing it.

\subsection{Motivation}

The design of a VAD is very sophisticated, given that its requirements are different from generic flow machines. For example, it needs low shear stress to minimize the damage to the blood cells. Also, the design should consider a non-Newtonian fluid model, given that they operate with blood. The operational conditions (pressure and mass flow) and size of the device imply that it operates under high Reynolds numbers, which indicates a turbulent regime. Thus, it is challenging to create a framework capable of systematically designing the VAD.

The use of the topology optimization method (TOM) is a feasible manner of creating custom designs systematically, given that it supports multi-objective functions. It has been successfully used to design non-Newtonian devices (PINGEN; MAUTE, 2010; ROMERO; N. Silva, 2017), to design turbulent flow devices (PAPOUTSIS-KIACHAGIAS; GIANNAKOGLOU, 2016; YOON, 2016), and it can create optimized topologies with relatively few information of traditional designs.

The TOM can also significantly reduce the development time, enabling the development of machines with different operational conditions. Thus, with a TOM framework, it is possible to create custom designs for each patient condition. Besides, it promotes higher 
flexibility and can achieve complex and non-intuitive geometries, which can now be built by using additive manufacturing techniques, such as the 3D printer.

\subsection{Scientific Contribution}

The scientific contribution consists mainly in the introduction of turbulent rotational flows to topology optimization, creation of a topology optimization model that considers non-Newtonian turbulent flows, and the development of a methodology based on TOM to design both the impeller and the volute of a VAD. Thus, the contributions are:

- Development of a topology optimization formulation using a turbulence model in a rotational frame;

- Development of a topology optimization formulation using a non-Newtonian turbulent formulation in a rotational frame;

- Definition of a Design Methodology for VAD Impeller and Volute based on TOM;

Four journal papers have been published:

- Design optimization of laminar flow machine rotors based on the topological derivative concept SÁ et al. 2017);

- Topology optimization method applied to laminar flow machine rotor design (SÁ et al., 2018);

- Topology optimization applied to the design of 2D swirl flow devices (ALONSO et al. 2018);

- Topology optimization based on a two-dimensional swirl flow model of Tesla-type pump devices (ALONSO et al. 2019)

\subsection{Document Outline}

This text presents the methodology to design impellers and volutes of VADs. The optimization results integrate the complete formulation of a turbulent non-Newtonian rotational flow. Initially, the mathematical and numerical models are shown, and the experimental setup is presented. Then, numerical results are analyzed. The optimized designs for the impeller and volute are built, and the prototypes are evaluated.

This document is organized as follows. In Chapter 2, the formulation used for the radial flow machine is described, as well as the non-newtonian effect formulation of the 
Carreu-Yasuda model. In Chapter 3, an introduction to turbulent flows is given, and the Reynolds Averaged Navier-Stokes equations with the Spalart-Allmaras model are presented with the necessary modifications that adequate the models to rotational frames. In this chapter, the Eikonal equation, which calculates the distance to the boundary, is introduced as well. In Chapter 4, the finite element model for solving the equations by using the weak-formulation approach is introduced. In Chapter 5, an introduction to topology optimization applied to fluid domains is given, and the material model, with the necessary changes in all state equations to account for its effect, are shown. There, the objective function is defined. In Chapter 7 , the numerical implementation is presented, the software framework is introduced, and the libraries in which the developed algorithm relies on are described. In Chapter 9 the experimental methodology and the equipment used are shown. The numerical results for the optimization of both the non-Newtonian and turbulent impellers are shown, as well as the optimized volutes. In Chapter 10, the experimental curves of pressure by mass flow are shown. Also, to further analyze the flow behavior, simulations of 3D models are shown and compared with the experimental data. Finally, in Chapter 11, a conclusion on the results is reported, and the suggested next steps of this topic are defined. 


\section{MODELING OF RADIAL FLOW MACHINE}

The model used in this work is based on the implementation performed by (ROMERO; SILVA, 2014), where the flow inside radial flow machines is modeled as a flow in a straight channel that rotates around a fixed-frame axis (inertial frame), as shown in Figure 2.1. Thus, the Navier-Stokes equations are considered with the addition of body forces representing the rotation terms imposed by the rotor.

Figure 2.1 - Impeller Model approximation by using a Rotational Straight Channel Flow.

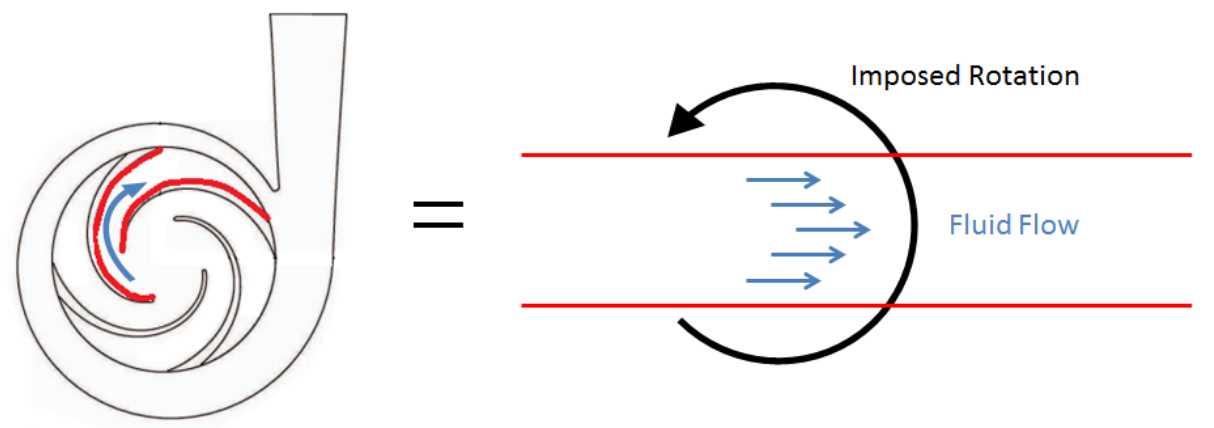

Adapted from Sá (2016)

The topology optimization method is used by considering only the flow field between blades, without considering the volute influence. Even though the fluid in a real flow machine is three-dimensional, for the case of radial centrifugal impellers, the axial velocity component can be neglected in comparison to the radial and tangential components. Hence the flow path can be approximated as a two-dimensional problem (ROMERO; SILVA, 2014).

\subsection{Equilibrium Equations}

Considering Newtonian flows, low Reynolds numbers and only steady state analysis, the incompressible Navier-Stokes equations are given by:

$$
\rho \nabla \mathbf{u} \cdot \mathbf{u}=-\nabla p+\mu \nabla \cdot\left(\nabla \mathbf{u}_{a}+\nabla \mathbf{u}_{a}^{T}\right)+\rho \mathbf{f}
$$

where $\mathbf{u}_{a}$ is the absolute velocity field given by $\mathbf{u}_{a}=\mathbf{u}+\boldsymbol{\omega} \times \mathbf{r}, p$ is the pressure, $\mu$ is the viscosity, and $\rho$ is the mass density. 
The imposed incompressibility simplifies the mass conservation equation to:

$$
\nabla \cdot \mathbf{u}_{a}=\mathbf{0} \text { in } \Omega
$$

The rotational reference system causes the introduction of terms related to the rotary motion of the fluid, such as the relative velocity $(\mathbf{u})$, and the Coriolis and Centrifugal body forces. Also, by introducing the term $\kappa(\alpha)$ representing domain porosity, the Brinkman model is obtained by adding a convection term (ROMERO; SILVA, 2014), given by:

$$
\rho \nabla \mathbf{u} \cdot \mathbf{u}=\nabla \cdot \mathbf{T}+\rho \mathbf{f}-2 \rho \boldsymbol{\omega} \times \mathbf{u}-\rho \boldsymbol{\omega} \times \boldsymbol{\omega} \times \mathbf{r}-\kappa(\alpha) \mathbf{u} \quad \text { in } \Omega
$$

where $\mathbf{T}=-p \mathbf{I}+\tau$ is the stress tensor, $\tau$ is the viscous stress tensor, given by: $\tau=$ $\mu\left(\nabla \mathbf{u}+\nabla \mathbf{u}^{T}\right)$. The term $\mathbf{u}$ is the relative velocity.

\subsection{Non-Newtonian Effects}

Blood is composed by cellular elements suspended in an aqueous solution called plasma. The cellular elements are red blood cells or erythrocytes, white blood cells or leukocytes and platelets or thrombocytes (GUYTON; HALL, 2006). Due to its microscopic structure the fluid properties changes with the flow conditions. Even though the density of blood may change locally with the flow, for some flow conditions it is possible to consider it as incompressible fluid (CHUONG; FUNG, 1984; KHURSHID; HOFFMANN, 2015). Hence, in this work, the incompressible assumption is used. Also, the literature indicates that it is more accurately represented when its viscosity is modeled as non-Newtonian fluid with shear-thinning behavior, where the dynamic viscosity $\mu$ is dependent of shear rate $\dot{\gamma}$, given by the following equation:

$$
\dot{\gamma}(\mathbf{u})=\sqrt{2 \epsilon(\mathbf{u}): \epsilon(\mathbf{u})}
$$

where the symbol : stands for the double scalar product and $\epsilon(\mathbf{u})$ is the velocity shear rate given by:

$$
\epsilon(\mathbf{u})=\nabla \mathbf{u}+\nabla \mathbf{u}^{T}
$$

Blood shear-thinning behavior is mainly related to following aspects of red blood cells: the propensity to form microstructures called rouleaux at low shear rates, their deformability, and the flow field alignment at high shear rates (ROBERTSON; SEQUEIRA; OWENS, 2009).

The blood viscosity model used here is the Carreau-Yasuda, which given by Equation 
2.6 (ROBERTSON; SEQUEIRA; OWENS, 2009):

$$
\mu(\dot{\gamma})=\mu_{\infty}+\left(\mu_{0}-\mu_{\infty}\right)\left[1+(\lambda \dot{\gamma})^{a}\right]^{\frac{n-1}{a}}
$$

where $\lambda=8.2$ is the time constant, $a=0.64$ is the Yasuda exponent, $n=0.2128$ is the power-law index, $\mu_{\infty}=0.0035 \mathrm{~Pa} \cdot s$ is the high shear viscosity, and $\mu_{0}=0.16 \mathrm{~Pa} \cdot s$ is the low shear viscosity (ABRAHAM; BEHR; HEINKENSCHLOSS, 2005). Thus, for zero shear rate, the viscosity presents a maximum value of $\mu_{0}=0.16$ Pa.s and for very high shear rates (approximately $>10^{3} \mathrm{~s}^{-1}$ ) the viscosity value converges to the same as the Newtonian model $\mu_{\infty}=0.0035 P$ a.s. This behavior is verified experimentally by Macosko (1994). It can be noticed that the chosen values for the constants make the model behave very similarly to the modified cross model presented in the literature (LEUPRECHT; PERKTOLD, 2001: ABRAHAM; BEHR; HEINKENSCHLOSS, 2005; ZHANG; LIU, 2015). The relation between viscosity and shear rate for blood in the models discussed are presented in Figure 2.2 .

Figure 2.2 - Shear rate dependant viscosity for Carreu-Yasuda model (logarithmic scale).

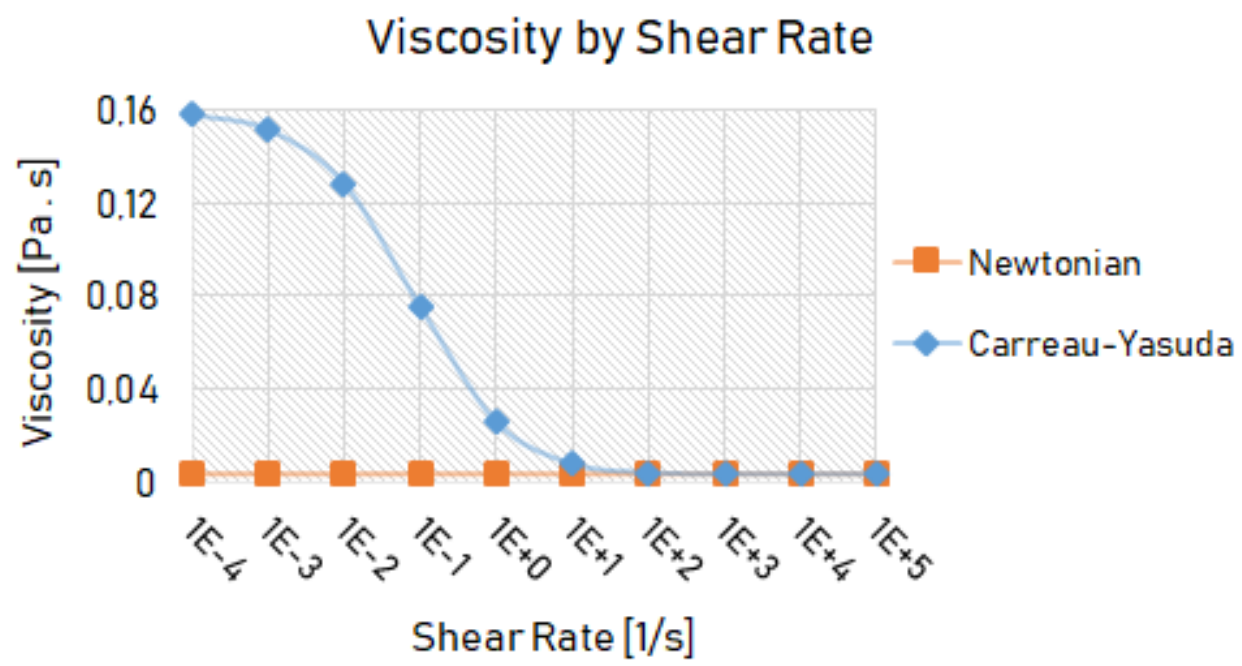




\section{TURBULENCE MODEL}

Almost all fluid flows encountered in daily life are turbulent, turbulence is the rule rather than the exception when studying fluid flows. Typical examples are flows around cars, airplanes, and buildings, the boundary layers and the wakes formed by the flow around those bodies are turbulent. Also, the flow and combustion in engines, both in piston engines and gas turbines, are highly turbulent. Thus, the correct understanding and modeling of turbulent flows are essential issues for engineering problems.

The solution of most flows can be achieved by using the Navier-Stokes equations. However, a precise prediction of complex and turbulent flows incurs high computational cost. This direct approach is defined as Direct Numerical Solution (DNS) (MOSER; KIM; MANSOUR, 1999) and can achieve accurate results when used with sufficient resolution of space and time scales. Thus, other approaches try to emulate the turbulence by adding models to the original Navier-Stokes equations, such as the Large Eddy Simulation (LES)(LYONS; HANRATTY; MCLAUGHLIN, 1991), which is a computationally more efficient approach that uses filtering techniques to solve the equations. A third way to solve these problems is the use of Reynolds Averaged Navier-Stokes (RANS) models, which implies in solving the incompressible Navier-Stokes equations with an extra set of transport equations for turbulent quantities (or turbulence models). The relationship between the computational cost and the degree of modeling used for each approach can be seen in Fig 3.1 .

Figure 3.1 - Degree of modelling versus Computational Cost.

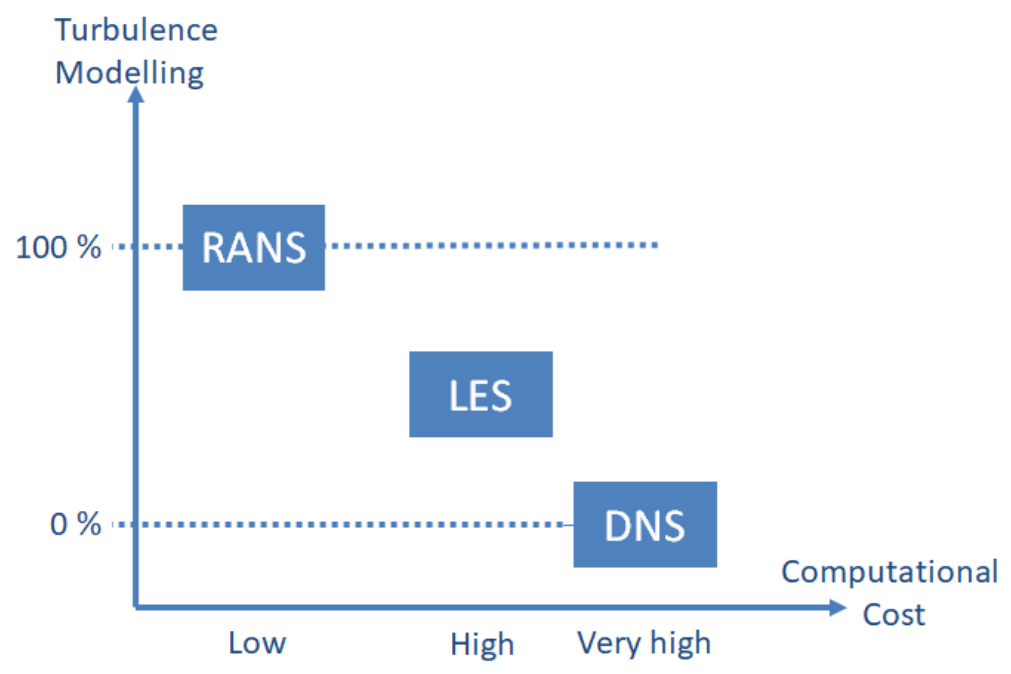


"A good turbulence model has to be extensive universality and not too complex to develop or use. Universality implies that a single set of empirical constants or functions, inserted into the equations, provides a close simulation of a large variety of types of flow. Complexity is measured by the number of differential equations which the model contains, and the number of the empirical constants and functions which are required to complete them. Increase in the first complicates the task of using the model because the model can become more unstable, increase in the second complicates the development" (LAUNDER; SPALDING, 1974). Thus, there are several different models to be used, and each one is more suitable for a different application. Most of these models are used to emulate a change in viscosity caused by the eddies, thus introducing a pseudo viscosity in the RANS equations, called eddy viscosity or turbulent viscosity.

The different models can be divided into three categories: Zero-equation models, One-equation models, and Two-equation (or more equations) models. This division takes into account the number of extra partial differential equations (PDEs) that need to be solved to achieve the final flow distribution.

The Zero-equation models (RODI, 1993) need just an algebraic (or geometric) relation to simulate the eddy viscosity, such as the mixing length model. The One-equation models use one extra PDE involving the eddy viscosity as a variable, for example, the Spalart-Allmaras (S-A) model (SPALART; ALLMARAS, 1992). The Two-equation models use two variables, in general, the kinetic energy of turbulence and turbulence dissipation, finally the eddy viscosity is defined as a relation between these variables, the most famous models in this category are the $k-\epsilon$ model (LAUNDER; SPALDING, 1974) and the $k-\omega$ model (WILCOX, 2008). There are also higher-order models with more equations, such as the $k-\epsilon-A$ and the $v^{2}-f$.

The mixing length models are easy to implement, and fast calculations are possible. Also, through experimental correlations for some well-known cases, this model can offer a reasonable prediction. However, these models are entirely incapable of considering flows where the turbulent length scale varies. The Spalart-Allmaras model has only one equation for the eddy viscosity; thus, it is economical and appropriate for flows with boundary layers of intermediary complexity. The standard $k-\epsilon$ is robust and reasonably accurate and suitable for long-accumulated performance data. However, for flow simulations with severe pressure gradients, streamline curvature or swirl and rotation, this model provides mediocre results. To overcome this limitation, more complex models were developed, RNG $k$ - $\epsilon$ or Realizable $k$ - $\epsilon$. Hence, the choice of the turbulence model can have a significant influence on the simulation accuracy (SPALART, 2000).

Usually, a more complete model is used when considering the flow inside a flow machine. However, this work focuses on using a turbulence model for topology optimization, in which the computational cost is a determining factor. Also, based on the effectiveness of using the 
Spalart-Allmaras in previous research (ZYMARIS et al., 2009, BUENO-OROVIO et al., 2012, ZHANG, 2012; YOON, 2016), this model will be used. Besides, some modifications increase the accuracy of the model under flows that present curvature and rotational domains (TAO et al. 2014), which will be described later. The work of Zhang et al. (2013) performs a comparison of different turbulence models operating with blood and concludes that the Spalart-Allmaras is not the best one but can predict the velocity field reasonably well.

The following sections present the Turbulence model equations. The Reynolds averaged Navier-Stokes is derived, and the Spalart-Allmaras model is shown with its variations.

\subsection{Reynolds Averaged Navier-Stokes equations (RANS)}

By decomposing the flow into a mean and fluctuating part, a time-average of the Navier- Stokes equations can be derived. With this Reynolds decomposition, the turbulent fluctuations of the flow can be defined in a single tensor, called the Reynolds stress tensor. Following the same approach presented by (YOON, 2016), the Reynolds decomposition for the velocity and pressure are defined as,

$$
\begin{aligned}
& \mathbf{u}(\mathbf{x}, t)=\overline{\mathbf{u}}(\mathbf{x})+\mathbf{u}^{\prime}(\mathbf{x}, t), \\
& p(\mathbf{x}, t)=\bar{p}(\mathbf{x})+p^{\prime}(\mathbf{x}, t)
\end{aligned}
$$

where $\overline{\mathbf{u}}$ and $\bar{p}$ are the statistically averaged quantities. $\mathbf{u}^{\prime}$ and $p^{\prime}$ are the fluctuation terms.

For an arbitrary time span, $T$, the integration of the fluctuating quantity can be assumed to be zero, and the time-averaged term is obtained from:

$$
\text { Reynolds-averaged quantities: } \overline{\mathbf{u}}(\mathbf{x})=\lim _{T \rightarrow \infty} \frac{1}{T} \int_{0}^{T} \mathbf{u}(\mathbf{x}, t) d t
$$

By inserting the above relationship between the time averaged term and the fluctuating term to the NS equation, we can define the following equation

$$
\begin{aligned}
& \rho(\overline{\mathbf{u}} \cdot \nabla) \overline{\mathbf{u}}=-\nabla \bar{p}+\nabla \cdot\left(\mu\left(\nabla \overline{\mathbf{u}}+\nabla \overline{\mathbf{u}}^{T}\right)\right)+\rho \nabla \cdot\left(-\overline{\mathbf{u}^{\prime} \mathbf{u}^{\prime}}\right) \text { in } \Omega \\
& \nabla \cdot \overline{\mathbf{u}}=0
\end{aligned}
$$

and the Reynolds stress is defined by:

$$
\tau_{R e}=-\rho \overline{\mathbf{u}^{\prime} \mathbf{u}^{\prime}}
$$

By using the Boussinesq hypothesis, i.e., the relationship between Reynolds stresses 
and velocity gradients through the scalar and isotropic eddy viscosity $\mu_{T}$ :

$$
\tau_{R e}=2 \mu_{T}\left(\nabla \overline{\mathbf{u}}+\nabla \overline{\mathbf{u}}^{T}\right)
$$

Finally, the RANS equations are given by:

$$
\begin{aligned}
& \rho(\overline{\mathbf{u}} \cdot \nabla) \overline{\mathbf{u}}=-\nabla \bar{p}+\nabla \cdot\left(\left(\mu+\mu_{T}\right)\left(\nabla \overline{\mathbf{u}}+\nabla \overline{\mathbf{u}}^{T}\right)\right) \\
& \nabla \cdot \overline{\mathbf{u}}=0
\end{aligned}
$$

A similar derivation can be applied to derive the RANS model of rotating domains and with the porosity model by using the relation:

$$
\overline{\mathbf{u}}=\overline{\mathbf{u}}_{\mathbf{r}}+\boldsymbol{\omega} \times \mathbf{r}
$$

Hence, by using the same procedure, the time-averaged version of Eq. 2.3 becomes:

$$
\rho \nabla \overline{\mathbf{u}}_{\mathbf{r}} \cdot \overline{\mathbf{u}}_{\mathbf{r}}=\nabla \cdot \mathbf{T}+\rho \mathbf{f}-2 \rho \boldsymbol{\omega} \times \overline{\mathbf{u}}_{\mathbf{r}}-\rho \boldsymbol{\omega} \times \boldsymbol{\omega} \times \mathbf{r}-\kappa(\alpha) \overline{\mathbf{u}}_{\mathbf{r}}
$$

where $\mathbf{T}=-\bar{p} \mathbf{I}+\left(\mu+\mu_{T}\right)\left(\nabla \overline{\mathbf{u}}_{\mathbf{r}}+\nabla \overline{\mathbf{u}}_{\mathbf{r}}^{T}\right)$. For simplicity, $\overline{\mathbf{u}}_{\mathbf{r}}$ and $\bar{p}$ are substituted by $\mathbf{u}$ and $p$ from here onward.

\subsection{Spalart-Allmaras turbulence model}

The Spalart-Allmaras (S-A) turbulence model, introduced in (SPALART; ALLMARAS 1992), is a popular choice for turbulence modeling due to its competitive accuracy and relatively low cost. The model directly computes the evolution of the eddy viscosity and is designed from empiricism and dimensional analysis. The S-A equation does not solve directly for the turbulent viscosity $\mu_{T}$, but rather for the modified turbulent viscosity $\nu_{t}$ which is used to compute the eddy viscosity. There are different variants for the S-A equation, here the baseline model, along with some of the variants, are presented.

\subsubsection{Baseline Model}

The eddy viscosity $\mu_{t}$ is given by (SPALART; ALLMARAS, 1992):

$$
\mu_{T}=\nu_{t} f_{v 1}, \quad f_{v 1}=\frac{\chi^{3}}{\chi^{3}+c_{v 1}^{3}}, \quad \chi=\frac{\nu_{t}}{\nu}
$$


where $\nu$ is the fluid kinematic viscosity. $\nu_{t}$ is the variable calculated by the S-A model and obeys the transport equation (considering the steady state):

$$
(\mathbf{u} \cdot \nabla) \nu_{t}=\overbrace{P-D}^{\text {Reaction }}+\frac{1}{\sigma_{\nu}}[\underbrace{\nabla \cdot\left(\left(\nu+\nu_{t}\right) \nabla \nu_{t}\right)}_{\text {Conservative Diffusion }}+\overbrace{c_{b 2}\left(\nabla \nu_{t}\right)^{2}}^{\text {Non-conservative Diffusion }}]
$$

where the production and wall destruction terms are

$$
P=c_{b 1}\left(1-f_{t 2}\right) \tilde{S} \nu_{t}, \quad D=\left(c_{w 1} f_{w}-\frac{c_{b 1}}{\kappa^{2}} f_{t 2}\right)\left[\frac{\nu_{t}}{d}\right]^{2}
$$

Here $\tilde{S}$ is the modified vorticity,

$$
\tilde{S}=S+\frac{\nu_{t}}{\kappa^{2} d^{2}} f_{v 2}, \quad f_{v 2}=1-\frac{\chi}{1+\chi f_{v 1}}
$$

where $S$ is the magnitude of vorticity and $d$ is the distance to the closest wall, and it is calculated by using the Eikonal equation,

$$
S=\sqrt{2 \Omega_{\mathbf{v}}: \Omega_{\mathbf{v}}}, \quad \Omega_{\mathbf{v}}=\frac{1}{2}\left(\nabla \mathbf{u}-\nabla \mathbf{u}^{T}\right)
$$

The function $f_{w}$ is

$$
f_{w}=g\left[\frac{1+c_{w 3}^{6}}{g^{6}+c_{w 3}^{6}}\right]^{\frac{1}{6}}, \quad g=r+c_{w 2}\left(r^{6}-r\right), \quad r=\min \left(\frac{\nu_{t}}{\tilde{S} \kappa^{2} d^{2}}, r_{l i m}\right) .
$$

Laminar suppression term is

$$
f_{t 2}=c_{t 3} \exp \left(-c_{t 4} \chi^{2}\right)
$$

The remaining constants are given by

$$
\begin{gathered}
c_{b 1}=0.1355, \quad \sigma_{\nu}=2 / 3, \quad c_{b 2}=0.622, \quad \kappa=0.41, \\
c_{w 1}=\frac{c_{b 1}}{\kappa^{2}+\left(1+c_{b 2}\right) / \sigma_{\nu}}, \quad c_{w 2}=0.3, \quad c_{w 3}=2, \\
c_{v 1}=7.1, \quad c_{t 3}=1.2, \quad c_{t 4}=0.5, \quad r_{l i m}=10
\end{gathered}
$$

The boundary conditions for $\nu_{t}$ are

$$
\text { no-slip wall }: \nu_{t}=0, \quad \text { symmetry plane }: \frac{\partial \nu_{t}}{\partial \mathbf{n}}=0
$$




\subsubsection{Preventing Negative Values of Modified Vorticity $\tilde{S}$}

According to Allmaras, Johnson and Spalart (2012), some modifications to the baseline model should be applied to avoid some numerical issues when solving the equations. There are different modifications possible; however, in this work, only the prevention of negative values on $\tilde{S}$ was necessary to obtain convergence. The modification takes place only in regions where $\tilde{S}<0.3 S$ and is given by:

$$
\begin{gathered}
\bar{S}=\frac{\nu_{t}}{\kappa^{2} d^{2}} f_{v 2} \\
\tilde{S}= \begin{cases}S+\bar{S} & : \bar{S} \geq-c_{v 2} S \\
S+\frac{S\left(c_{v 2}^{2} S+c_{v 3} \bar{S}\right)}{\left(c_{v 3}-2 c_{v 2}\right) S-\bar{S}} & : \bar{S}<-c_{v 2} S\end{cases}
\end{gathered}
$$

with $c_{v 2}=0.7$ and $c_{v 3}=0.9$.

\subsubsection{Rotation/Curvature Correction (RC)}

When rotational domains are considered, it is argued by Spalart and Shur (1997) that a correction in the turbulence production term is needed. The work of Tao et al. (2014) shows that RANS simulations without corrections, present an isotropic model for the turbulence and is not enough to describe some turbulence effects, such as flow separation. The rotation correction terms supplement the model with anisotropy. Also, it is shown that this correction improves the simulation accuracy by comparing it with experimental data of a centrifugal impeller. The corrected model is described as follows.

The first correction proposed consists of multiplying the $P$ (Eq. 3.14 by an empirical rotation function $f_{r 1}$ (SPALART; SHUR, 1997):

$$
f_{r 1}\left(r^{*}, \tilde{r}\right)=\left(1+c_{r 1} \frac{2 r^{*}}{1+r^{*}}\left(1-c_{r 3} \tan ^{-1}\left(c_{r 2} \tilde{r}\right)\right)-c_{r 1}\right.
$$

where the constants are $c_{r 1}=1, c_{r 2}=12, c_{r 3}=1 . \tilde{r}$ and $r^{*}$ are functions of the strain rate $\left(S_{s r}\right)$ and the vorticity corrected by rotation $\left(W_{v r}\right)$ :

$$
\begin{gathered}
S_{R C}=\sqrt{2 \mathbf{S}_{s r}: \mathbf{S}_{s r}}, \quad \mathbf{S}_{s r}=\frac{1}{2}\left(\nabla \mathbf{u}+\nabla \mathbf{u}^{T}\right) \\
W_{R C}=\sqrt{2 \mathbf{W}_{v r}: \mathbf{W}_{v r}}, \quad \mathbf{W}_{v r}=\frac{1}{2}\left[\left(\nabla \mathbf{u}-\nabla \mathbf{u}^{T}\right)+2 \boldsymbol{\omega}_{r o t}^{*}\right]
\end{gathered}
$$

where $\boldsymbol{\omega}_{\text {rot }}^{*}$ is the resulting tensor, given in index notation by:

$$
\boldsymbol{\omega}_{r o t}^{*}=\omega_{\text {rot } j i^{*}}^{*}=\epsilon_{m j i} \omega_{m}
$$


with $\omega_{m}=\left[\omega_{x}, \omega_{y}, \omega_{z}\right]$ being the frame rotation. The tensor in vector notation is

$$
\boldsymbol{\omega}_{\text {rot }}^{*}=\left[\begin{array}{ccc}
0 & \epsilon_{321} \omega_{z} & \epsilon_{231} \omega_{y} \\
\epsilon_{312} \omega_{z} & 0 & \epsilon_{132} \omega_{x} \\
\epsilon_{213} \omega_{y} & \epsilon_{123} \omega_{x} & 0
\end{array}\right]=\left[\begin{array}{ccc}
0 & -\omega_{z} & \omega_{y} \\
\omega_{z} & 0 & -\omega_{x} \\
-\omega_{y} & \omega_{x} & 0
\end{array}\right]
$$

In a more recent work Zhang et al. (2013) propose a modification to simplify the original rotation function (Eq. 3.22 by redefining $\tilde{r}$ :

$$
r^{*}=\frac{S_{R C}}{W_{R C}}, \quad \tilde{r}=\frac{W_{R C}}{S_{R C}}\left(\frac{W_{R C}}{S_{R C}}-1\right)
$$

This final model with curvature/rotation correction is the one used in this work for modeling the turbulence inside the rotational domain. For the topology optimization, another modification is necessary, which consists in introducing a penalization term similar to the Brinkman equation to remove the eddy viscosity in solid regions. This modification is presented in Chapter 5 .

\subsection{Eikonal Equation}

The Spalart-Allmaras model uses the distance to the nearest wall in the equation. The wall distance can be calculated by iterating the mesh and using search procedures. However, this procedure can become very costly for refined meshes (TUCKER et al. 2005). One way to circumvent this is by using differential equations to calculate all distances in one solve. Different equations describe the wall distance and can be seen in Tucker et al. (2005). In this work, a hyperbolic Eikonal equation is used:

$$
|\nabla \phi|=1+\lambda_{e i k} \nabla^{2} \phi
$$

where $\phi$ is the distance to the nearest wall and $\lambda_{e i k}$ is an emulated viscosity to smooth the solution. For this equation to represent the wall distance, it is solved considering that $\phi=0$ at the boundaries.

Figure 3.2 shows an example of the Eikonal equation behavior. The value of $\phi$ represents the distance to the nearest wall, i.e., for the point $P_{1}$ the value $\phi_{1}$ is the distance to the wall at the right, while for the point $P_{2}$, the value $\phi_{2}$ is the distance to the bottom wall. 
Figure 3.2 - Distance to the nearest wall (Eikonal example).

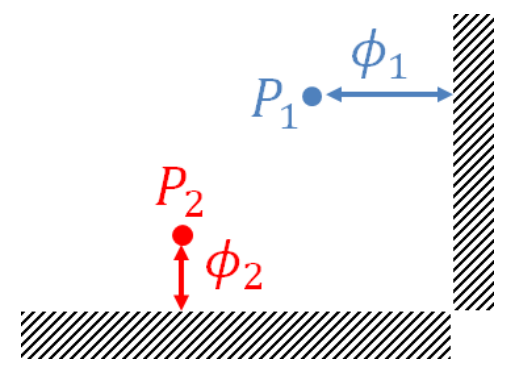

Equation 3.28 also needs to be modified to address the material model in the topology optimization, which consists in introducing a penalization term to guide the wall distance to zero at solid regions. This modification is presented in Chapter 5. 


\section{FINITE ELEMENT METHOD APPLIED TO FLOW MACHINES}

The analytical solutions of the partial differential equations (PDE) shown on the previous sections are only know for very simple problems. However, when solving complex problems, it is possible to use a numerical approach in order to obtain an approximate solution that is subjected to precision criteria and computational cost. Hence, it is plausible to use a solution method such as the Finite Element Method (FEM) or the Finite Volume Method (FVM). It is usual for commercial software to use the FVM, however in this work, the FEM is chosen, given that it is more often seen in the literature with topology optimization applications.

In order to solve the system of equations, the weighted residuals procedure of Galerkin's method is applied to the weak formulation of a Navier-Stokes problem in a rotating reference system.

A weighted residual is obtained by multiplying the original PDE by a test function and then integrating the inner product over the domain. Using the Galerkin's method means that the unknown field is represented by the same function space used for the weight functions.

\subsection{Rotational Navier-Stokes Weak Formulation}

The weak formulation for the flow problem, involving Navier-Stokes equations together with a porous media flow with non-newtonian fluid, in a rotating reference system considering the steady state is given by (ROMERO; SILVA, 2014):

$$
\begin{gathered}
R_{c}=\int_{\Omega}(\nabla \cdot \mathbf{u}) q d \Omega=0 \\
R_{m}=\int_{\Omega}(\rho \mathbf{u} \nabla \mathbf{u}) \cdot \mathbf{v} d \Omega+\int_{\Omega} \kappa(\mathbf{x}) \mathbf{v} \cdot \mathbf{u} d \Omega+\int_{\Omega} \mathbf{T}(\mathbf{u}): \nabla \mathbf{v} d \Omega \\
+\int_{\Omega} \mathbf{v}[2 \rho(\boldsymbol{\omega} \times \mathbf{u})+\rho \boldsymbol{\omega} \times(\boldsymbol{\omega} \times \mathbf{r})] d \Omega-\int_{\Omega} \mathbf{b} \cdot \mathbf{v} d \Omega-\int_{\Gamma}(\mathbf{T}(\mathbf{u}) \cdot \mathbf{n}) \cdot \mathbf{v} d \Gamma=0,
\end{gathered}
$$

where $\mathbf{v}$ and $q$ are the test functions, $q$ is a scalar function, $\mathbf{v}$ is a vector function and $\mathbf{b}$ represents the external body forces, such as gravity. The Galerkin's method is applied by choosing the test functions as the weighting functions, so $\mathbf{v}=\Phi_{i}(\mathbf{x})$ and $q=\chi_{i}(\mathbf{x})$. The weighting function $\Phi_{i}$ associated with the momentum equations is bi-quadratic and $\chi_{i}$ 
associated to continuity equation is linear.

The stress tensor $\mathbf{T}(\mathbf{u})$ is a function of $\mathbf{u}$ when a non-newtonian fluid is considered, and is given by:

$$
\mathbf{T}(\mathbf{u})=\left[\begin{array}{cc}
-p+2 \mu(\dot{\gamma}) \frac{\partial u_{1}}{\partial x_{1}} & \mu(\dot{\gamma})\left(\frac{\partial u_{1}}{\partial x_{2}}+\frac{\partial u_{2}}{\partial x_{1}}\right) \\
\mu(\dot{\gamma})\left(\frac{\partial u_{1}}{\partial x_{2}}+\frac{\partial u_{2}}{\partial x_{1}}\right) & -p+2 \mu(\dot{\gamma}) \frac{\partial u_{2}}{\partial x_{2}}
\end{array}\right]
$$

where $\mu(\dot{\gamma})$ is given by the Carreu-Yasuda model (Eq. 2.6).

The finite element method is implemented by using Taylor-Hood elements, where the fluid velocity and pressure have different interpolation ranks. In this case, the velocity has a quadratic interpolation, and pressure has a linear interpolation, as shown in Fig. 4.1. This is done to avoid instability issues in the solution, given that this element interpolation combination addresses the "Ladyzhenskaya-Babuska-Brezzi (LBB) condition". The design variable is defined at each node with linear interpolation.

Figure 4.1 - Element Interpolation for Navier-Stokes Eq.
(a) Velocity.
(b) Pressure.
(c) Design Variable.
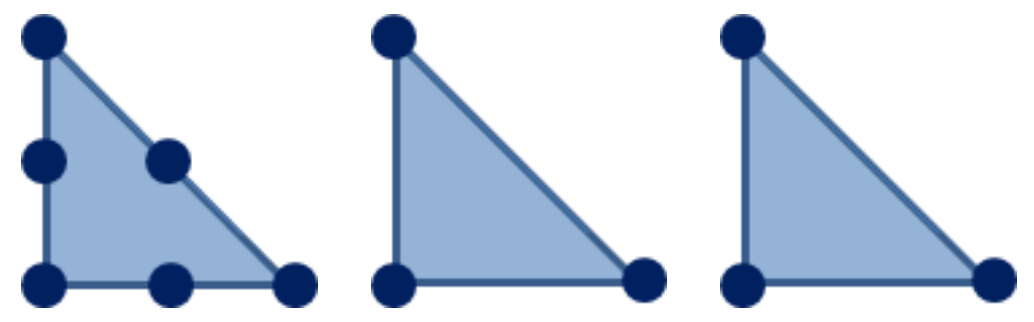

\subsection{Rotational RANS Weak Formulation}

The RANS weak form is very similar to the Navier-Stokes equation, however, the meaning of the calculated terms are very different. The formulation for the RANS weak formulation is given by:

$$
\begin{gathered}
R_{R A N S c}=\int_{\Omega}(\nabla \cdot \mathbf{u}) q d \Omega=0 \\
R_{R A N S m}=\int_{\Omega}(\rho \mathbf{u} \nabla \mathbf{u}) \cdot \mathbf{v} d \Omega+\int_{\Omega} \kappa(\mathbf{x}) \mathbf{v} \cdot \mathbf{u} d \Omega+\int_{\Omega} \mathbf{T}\left(\mu_{T}\right): \nabla \mathbf{v} d \Omega \\
+\int_{\Omega} \mathbf{v}[2 \rho(\boldsymbol{\omega} \times \mathbf{u})+\rho \boldsymbol{\omega} \times(\boldsymbol{\omega} \times \mathbf{r})] d \Omega-\int_{\Omega} \mathbf{b} \cdot \mathbf{v} d \Omega-\int_{\Gamma}\left(\mathbf{T}\left(\mu_{T}\right) \cdot \mathbf{n}\right) \cdot \mathbf{v} d \Gamma=0,
\end{gathered}
$$

where $\mathbf{T}\left(\mu_{T}\right)=-p \mathbf{I}+\left(\mu+\mu_{T}\right)\left(\nabla \mathbf{u}+\nabla \mathbf{u}^{T}\right)$ and $\mu_{T}$ is given by Eq. 3.12. Notice that when the non-Newtonian fluid is considered the fluid molecular viscosity $(\mu)$ becomes the non-Newtonian viscosity $(\mu(\dot{\gamma}))$. 


\subsection{Spalart-Allmaras Weak Formulation}

The weak formulation for the Spalart-Allmaras equation is obtained, in a similar way as before, by multiplying the strong form by a test function and integrating by parts, and is given by:

$$
\begin{aligned}
R_{S A}=\int_{\Omega}\left[(\mathbf{u} \cdot \nabla) \nu_{t} \lambda_{S A}-f_{r 1} P+D-\frac{1}{\sigma_{\nu}} c_{b 2}\left(\nabla \nu_{t}\right)^{2}\right] \lambda_{S A} d \Omega \\
\quad+\int_{\Omega} \frac{1}{\sigma_{\nu}}\left(\nu+\nu_{t}\right) \nabla \nu_{t} \cdot \nabla \lambda_{S A} d \Omega-\int_{\Gamma} \frac{1}{\sigma_{\nu}}\left(\nu+\nu_{t}\right) \lambda_{S A} \nabla \nu_{t} \cdot \mathbf{n} d \Gamma=0
\end{aligned}
$$

where $\lambda_{S A}$ is the test function, $P$ and $D$ are given by $3.14, \mathbf{n}$ is normal vector, $f_{r 1}$ is the Rotation correction term and the remaining constants are defined in Sec 3.2.1. The element is interpolated with a quadratic function, as shown in Fig.4.2

Figure 4.2 - Element Interpolation for $\nu_{t}$ from the Spalart-Allmaras Eq.

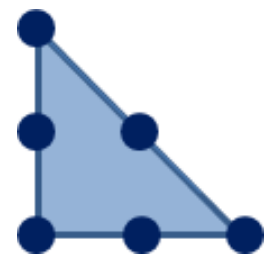

\subsection{Eikonal Weak Formulation}

On following the same procedure as for Eq. 3.28 , and integrating by parts, the weak formulation is given by:

$$
R_{e i k}=\int_{\Omega} \sqrt{\nabla \phi \cdot \nabla \phi} y_{e}-f_{e} y_{e}+\lambda_{e i k} \nabla \phi \cdot \nabla y_{e} d \Omega-\int_{\Gamma} y_{e} \nabla \phi \cdot \mathbf{n} d \Gamma
$$

where $f_{e}=1, y_{e}$ is the test function, $\lambda_{e i k}$ is the emulated viscosity (Eq. 3.28) and $\mathbf{n}$ is the normal vector. Again, the element is interpolated with a quadratic function, as shown in Fig. 4.3 .

Figure 4.3 - Element Interpolation for $\phi$ from the Eikonal Eq.

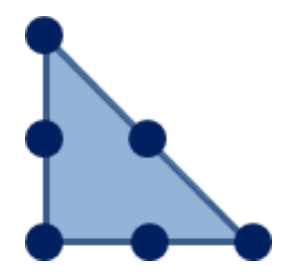




\section{TOPOLOGY OPTIMIZATION METHOD}

\subsection{Topology Optimization Method applied to Fluid Flow Problems}

In the topology optimization method, material is distributed (fluid or solid) over a domain, aiming to maximize (or minimize) an objective function under determined constraints. This method was first introduced to fluid domains by Borrvall and Petersson (2003), where they apply the technique to 2D flow channel problems, aiming to minimize the energy dissipation over the domain. In that case, the flow was modeled by using the Stokes equations for incompressible flows, without considering body forces, and with low Reynolds numbers.

Gersborg-Hansen (2003), Gersborg-Hansen, Sigmund and Haber (2005) and Olesen, Okkels and Bruus (2006) extended this approach by using Navier-Stokes equations for a more extensive Reynolds number range and considering additional effects, such as body force and non-linearities. Besides that, the work of Gersborg-Hansen also investigated different objective functions, other than energy dissipation, so with their implementation, it is possible to design systems based on velocity variation across the channel, such as directional flow selectors, in which the path chosen by the fluid depends on its velocity.

Evgrafov (2005) reassesses the work of Borrvall and Petersson (2003) and compares the Brinkman model used with a different approach by considering the fluid viscosity as a problem variable. Evgrafov also studied the application of topology optimization to slightly compressible fluid (EVGRAFOV, 2004). The effects of non-newtonian fluids over the topology optimization was evaluated by Pingen and Maute (2010).

Another approach to fluid optimization was developed by Wiker, Klarbring and Borrvall (2007), in which a specific region optimization separates the distribution of material under Stokes flow and porous media (Darcy flow). Thus, the resulting topology has regions where only fluid is present (Stokes flow) and regions where a porous media is established.

In the work of Deng et al. (2013), the topology optimization method is applied to the flow channel problem, aiming to minimize the pressure loss, considering Navier-Stokes formulation and the presence of body forces. They solve the system problem with the adjoint method, and implemented the software using the commercial software COMSOL Multiphysics. Another work aiming at the optimization of flow channels is Sá et al. (2016), in which they use a topological derivative method to obtain the topologies of fluid flow channels.

The topology optimization method applied to flow machines has been initially studied 
in the work Romero and Silva (2014), in which the fluid flow in the device is modeled as Navier-Stokes flow with the addition of a rotating reference system, giving rise to the effects of Centrifugal force and Coriolis force. In a similar work, Sá et al. (2017) performed the rotor optimization of Newtonian pumps by using an optimization based on the topological derivative concept. In their work various configurations are proposed for the machine rotor, exploring the influence of the initial domain and the effects of changes in the boundary conditions. As a result, non-intuitive geometries, which differ from traditional geometries, are obtained.

Recently, researchers have shown interest in applying the topology optimization method to design devices that conduct blood by considering the non-Newtonian effects present in this fluid. One of the approaches, proposed by Hyun, Wang and Yang (2014), optimizes the fluid flow channel through the Navier-Stokes equations, where a multi-objective function is defined by relating the reduction of wall shear stress and viscous energy dissipation through the use of different weights.

Focusing in devices operating with blood, such as arterial bypasses, the work of Zhang and Liu (2015) performs an optimization by using a level-set method to minimize flow shear stress, and compares the results with Abraham, Behr and Heinkenschloss (2005), that applied shape optimization to the same problem.

The topology optimization method applied to flow machines with non-newtonian fluids also has been recently studied in the work of Romero and N. Silva (2017), in which they present the differences between designing a rotor with a Newtonian model and non-newtonian model, in this case, a modified cross model.

When we consider flow machines operating in real-life applications, the flow becomes more complex and almost always present turbulent behaviors. Thus, in order to improve the current model, it is necessary to include a turbulence model to topology optimization. The work of Yoon (2016) is one of the first works to considers a turbulence model in topology optimization. It presents the optimization of flows inside channels for different Reynolds numbers by using the Spalart-Allmaras model. Other recent works also show the inclusion of the $k-\omega$ model in topology optimization (DILGEN et al., 2018a; DILGEN et al. 2018b).

\subsection{Topology Optimization Model}

\subsubsection{Material Model}

The topology optimization method involves distributing material over a specified domain by following certain requisites. Therefore, in order to determine the path to be followed by the fluid flow and to define a design variable to perform a topology 
optimization, a porous domain was introduced by Borrvall and Petersson (2003), dividing domain regions between high permeability material, interpreted as pure fluid, and low permeability material, representing solid, i.e., the porosity virtually separates fluid and solid regions (GERSBORG-HANSEN, 2003). This is done with the introduction of the absorption term $\kappa$ that controls the material distribution in the domain.

The absorption coefficient $\kappa$ can be considered as a interpolation of the material flux on the domain, which represents the transition of material between high porosity (solid, $\kappa \gg 1$ ) and low porosity (fluid, $\kappa=0$ ) regions. This coefficient is a function of $\alpha$, a pseudo-density field which is the optimization problem design variable. The choice of this function $\alpha \rightarrow \kappa(\alpha)$ allows the design variable to assume intermediate values, between 0 - 1. So, to suppress these undesired values a convex and q-parameterized interpolation function can be chosen (BORRVALL; PETERSSON, 2003):

$$
\kappa(\alpha)=\kappa_{\max }+\left(\kappa_{\min }-\kappa_{\max }\right) \alpha \frac{1+q}{\alpha+q}
$$

with $\kappa \in\left[\kappa_{\min }, \kappa_{\max }\right], \alpha$ represents points inside the domain and $q$ is a parameter that controls $\kappa$ curvature. Thus, for $q \rightarrow \inf , \kappa \rightarrow \kappa_{\max }+\left(\kappa_{\min }-\kappa_{\max }\right) \alpha$ is a linear function. Figure 5.1 illustrates this behavior by considering $\kappa_{\max }=10,000$ and $\kappa_{\min }=0$. The optimization process aims to obtain values of 0 or 1 to the design variable $\alpha(\alpha \approx 0$ or $\alpha \approx 1$ ), because a intermediate value would not have a physical meaning. Therefore, when $\alpha \approx 1 \Rightarrow \kappa=\kappa_{\min }$, represents a flow of pure fluid, while when $\alpha \approx 0 \Rightarrow \kappa=\kappa_{\max }$, represents a restricted flow inside a porous media.

Figure $5.1-\kappa$ as function of parameters $\alpha$ and $q$.

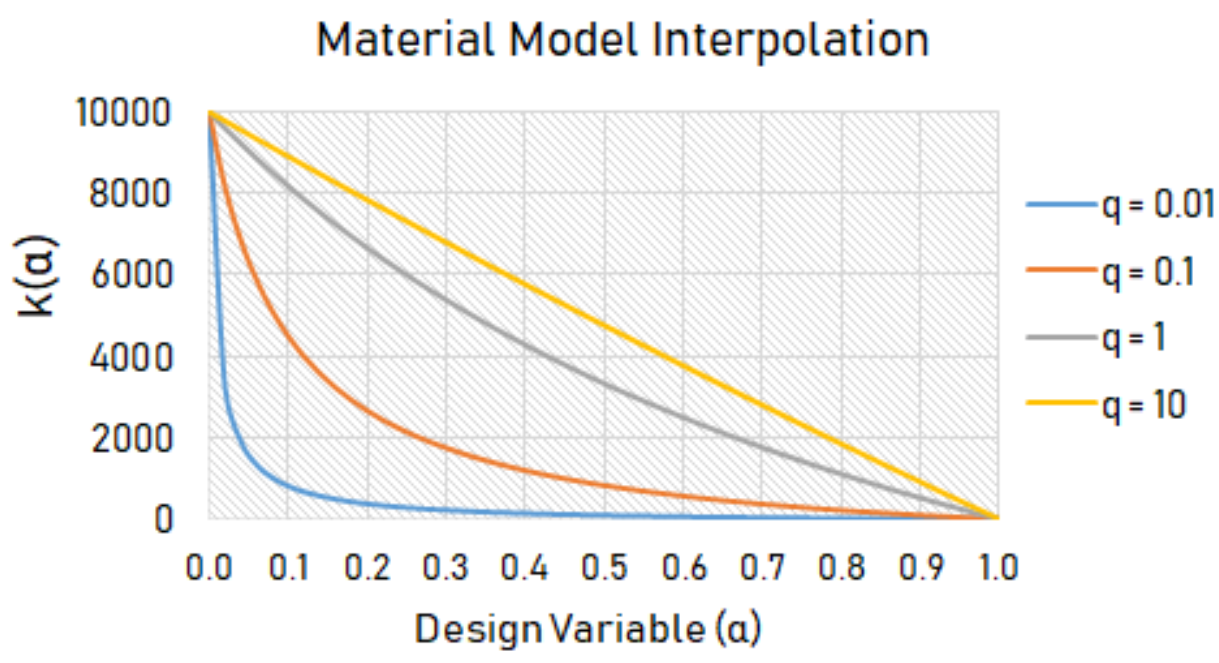




\subsubsection{Non-Newtonian Equation with Material Model}

Some works in the literature (PINGEN; MAUTE, 2010, HYUN; WANG; YANG, 2014) suggest that the non-Newtonian viscosity should be interpolated with the design variable, in order to reduce the non-Newtonian effect in areas of high porosity (solid), and avoid convergence problems. Thus, the viscosity also is interpolated as a function of the pseudo-density $\alpha$.

Hyun, Wang and Yang (2014) proposed a model where viscosity is interpolated with the same penalty parameter $q$ used for the inverse permeability (Eq. 5.1). In this work, the interpolation values are changed, and the model is given by:

$$
\mu(\alpha)=\mu_{s}+\left(\mu_{f}-\mu_{s}\right) \frac{\alpha(1+q)}{\alpha+q}
$$

where $\mu_{f}$ is the shear rate dependent viscosity $\mu(\dot{\gamma})$ (Eq. 2.6) and $\mu_{s}$ is equal to the Newtonian viscosity $\left(\mu_{\infty}=0.0035\right.$ Pa.s $)$. This behavior is similar to the one presented by Pingen and Maute (2010).

\subsubsection{Spalart-Allmaras Equation with Material Model}

The work of Yoon (2016) uses an exponential function in order to account for the material model in the Spalart-Allmaras equations, by adding a penalty term consisting of a big constant and the design variable powered by a constant $\left(\alpha^{n}\right)$. However, in this work, the added term is similar to the friction force on Navier-Stokes eq., i.e., the interpolation function of Eq. 5.1 is used in Eq. 4.6. The result is given by:

$$
\begin{aligned}
R_{S A} & =\int_{\Omega}\left[(\mathbf{u} \cdot \nabla) \nu_{t} \lambda_{S A}-f_{r 1} P+D-\frac{1}{\sigma_{\nu}} c_{b 2}\left(\nabla \nu_{t}\right)^{2}\right] \lambda_{S A} d \Omega \\
& +\int_{\Omega} \frac{1}{\sigma_{\nu}}\left(\nu+\nu_{t}\right) \nabla \nu_{t} \cdot \nabla \lambda_{S A} d \Omega-\int_{\Gamma} \frac{1}{\sigma_{\nu}}\left(\nu+\nu_{t}\right) \lambda_{S A} \nabla \nu_{t} \cdot \mathbf{n} d \Gamma \\
& +\int_{\Omega} \kappa_{S A}(\alpha) \nu_{t} \lambda_{S A} d \Omega
\end{aligned}
$$

where $\kappa_{S A}(\alpha)$ is a function similar to Eq. 5.1 but with different constants. The remaining terms are defined in Eq. 4.6.

\subsubsection{Eikonal Equation with Material Model}

The Spalart-Allmaras model needs the distance to the nearest wall to calculate the eddy viscosity. The Eikonal equation presented in Sec. 3.3 is used to calculate the distance of each point of the domain to the nearest wall. However, in reality, the equation calculates the distance to the nearest node with zero value, hence, defining the boundary condition as zero gives the distance to the boundary. The addition of the material model to the equation 
can make new nodes to assume the value zero and, therefore, making it to calculate the distance to the nearest "solid" point.

Hence, the same idea of a material model that changes the calculated value at a point is used for the Eikonal equation (Eq. 4.7), by including the interpolation function multiplied by the wall distance resulting in:

$$
R_{e i k}=\int_{\Omega} \sqrt{\nabla \phi \cdot \nabla \phi} y_{e}-f_{e} y_{e}+\lambda_{e i k} \nabla \phi \cdot \nabla y_{e} d \Omega-\int_{\Gamma} y_{e} \nabla \phi \cdot \mathbf{n} d \Gamma+\int_{\Omega} \kappa_{e i k}(\alpha) \phi y_{e} d \Omega
$$

where $f_{e}=1$ and $\kappa_{e i k}(\alpha)$ is a function similar to Eq. 5.1 but with different constants.

In order to exemplify the effect of the material model, the following case is analyzed (Fig. 5.2).

An L-shaped domain is chosen with the dimensions shown in Fig. 5.2a. The design variable is distributed in the domain with regions with value 1.0, representing fluid (red), i.e., regions where the distance needs to be calculated, and regions with value 0.0 , representing solid (blue), where the distance should be set to zero. Also, a region with an intermediary value of 0.5 (gray) is defined.

Figure 5.2 - L-shape Example.

(a) Domain.

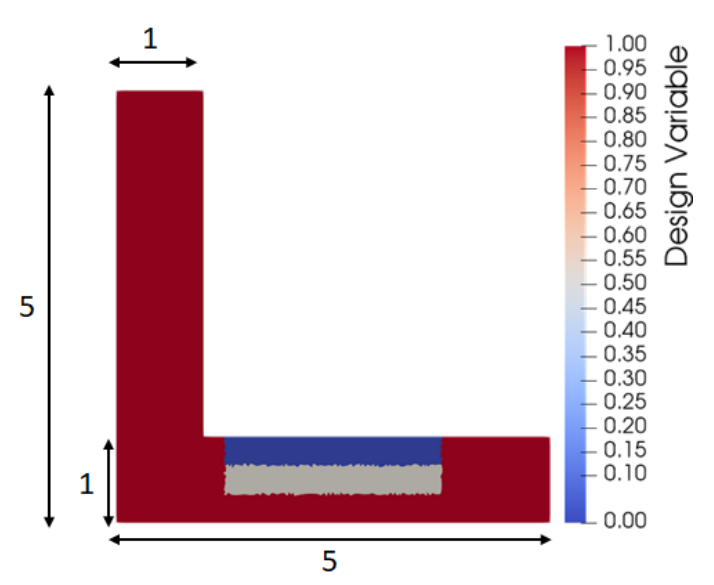

(b) Wall Distance.

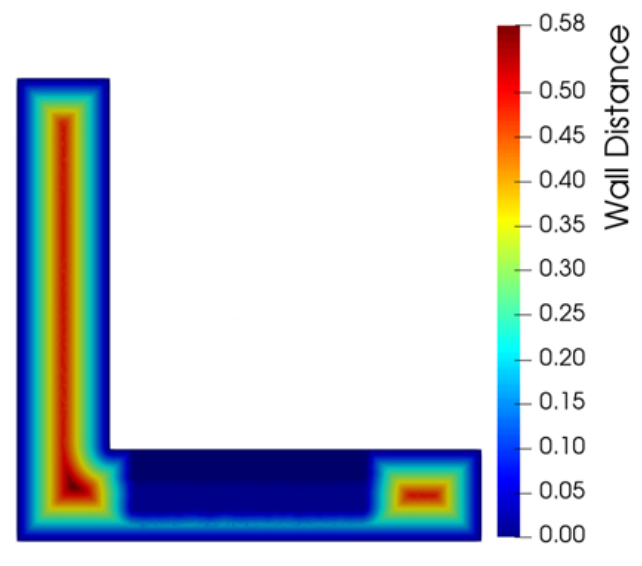

Figure $5.2 \mathrm{~b}$ shows the calculated distance for this configuration. It can be noticed that at the vertical part, the distance is calculated as the distance to the nearest boundary (wall). A point at the middle of this area has the wall distance equal to 0.5 , given that the width is 1.0. At the horizontal part, the solution behaves differently, calculating the distance to the nearest solid region.

Performing a cut at the horizontal region (Fig. 5.3a) and analyzing the distance behavior (Fig. 5.3b), one can see that for $y$ between 0.0 and 0.3 (fluid region - red region) the distance is calculated without the interference of the gray region, i.e., the height is 0.3 and the value of a node at the middle of the red region $(y=0.15)$ is around 0.15 . The value 
is not exactly 0.15 due to the influence of the gray region. At the gray region, the distance is damped, and an intermediary distance value is held (around 0.02). At the solid region the distance result is in fact zero. Thus, with the proposed material model, it is possible to emulate a wall and calculate the correct distance by using the design variable.

Figure 5.3 - Horizontal Region Analyze.

(a) Cut.

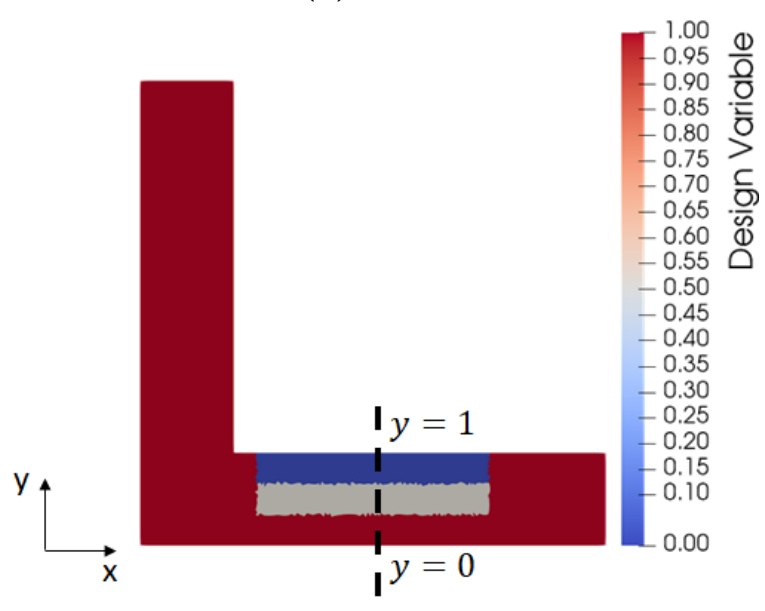

(b) Wall Distance over the Line.

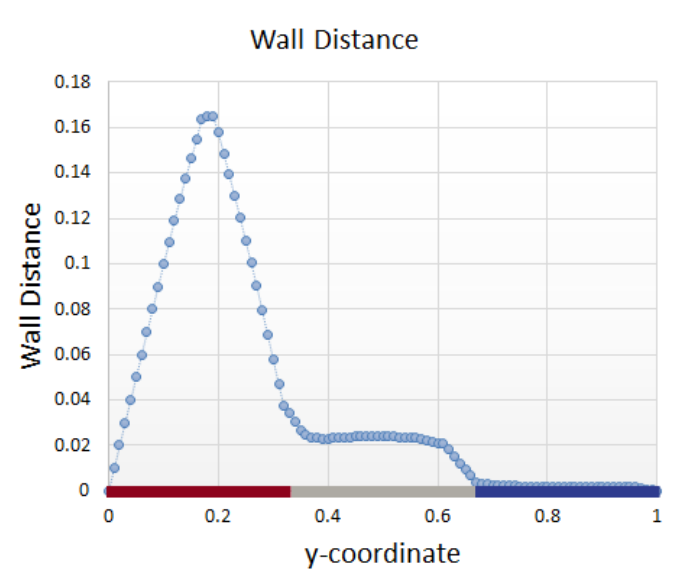

\subsection{Topology Optimization Problem}

The optimization problem in this work has the following formulation

$$
\begin{aligned}
\text { Minimize : } & c(\mathbf{z}(\alpha), \alpha) \\
\alpha & \\
\text { subjected to : } & \mathbf{g}(\mathbf{z}(\alpha), \alpha) \leq 0 \\
& \mathbf{r}(\mathbf{z}(\alpha), \alpha)=0 \\
& \alpha_{\min } \leq \alpha \leq \alpha_{\max }
\end{aligned}
$$

The function $c(\mathbf{z}(\alpha), \alpha)$ is a multi-objective that includes minimization of energy dissipation and minimization of vorticity.

Besides, some equality constraints are defined on Equation 5.7, which consists of the constitutive equations (Navier-Stokes, Spalart-Allmaras and Eikonal eqs.). A volume constraint is defined to constrain the amount of fluid regions on the domain, so a fraction of the domain volume $(f|\Omega|)$ is defined as an upper bound to create regions of fluid and leaving the remainder to be occupied by solid. Thus,

$$
\int_{\Omega} \alpha d \Omega \leq f|\Omega|
$$

where $f$ is the prescribed volume fraction, a constant between 0 and 1 , and $|\Omega|$ is the total 
volume of the domain (or total area in the $2 \mathrm{D}$ case).

\subsubsection{Energy Dissipation}

The shear stress functions are directly related to hemolysis of the blood (GHATTAS; HE; ANTAKI, 1995) and the energy dissipation functional has a high correlation with the shear stress, given that both are directly proportional to the velocity shear rate. Thus, minimizing energy dissipation is an indirect way to minimize hemolysis.

Minimizing the energy dissipation is one of the objective functions in this work, it represents the loss in fluid potential power, and it is obtained through the principle of virtual work applied to the equilibrium equations. Also, minimizing the dissipated power in the fluid is equivalent to reduce the average pressure drop, under the assumption that velocities are uniform and normal to the domain boundary (BORRVALL; PETERSSON 2003). Borvall and Petersson proposed a form for the energy dissipation that considers the effect of material model, given by:

$$
E(\alpha)=\int_{\Omega}\left[\frac{1}{2} \mu\left(\nabla \mathbf{u}+\nabla \mathbf{u}^{T}\right):\left(\nabla \mathbf{u}+\nabla \mathbf{u}^{T}\right)+\kappa(\alpha) \mathbf{u}^{2}\right] d \Omega
$$

where $\mathbf{u}$ is the relative velocity field on the rotating system, $\mu$ is the fluid molecular viscosity, and $\alpha$ is the design variable.

The energy dissipation can be divided into two parts, defined in this work as Viscous Dissipation and Friction Dissipation. The viscous dissipation is given by:

$$
E_{v i s c}=\int_{\Omega}\left[\frac{1}{2} \mu\left(\nabla \mathbf{u}+\nabla \mathbf{u}^{T}\right):\left(\nabla \mathbf{u}+\nabla \mathbf{u}^{T}\right)\right] d \Omega
$$

The second part, the Friction Dissipation, is given by:

$$
E_{\text {fric }}=\int_{\Omega} \kappa(\alpha) \mathbf{u}^{2} d \Omega
$$

The viscosity present in this functional can vary depending on the model used (non-Newtonian, turbulence, or both). The energy dissipation formulation for the non-Newtonian case is given by:

$$
E_{n n}(\alpha)=\int_{\Omega}\left[\frac{1}{2} \mu(\dot{\gamma})\left(\nabla \mathbf{u}+\nabla \mathbf{u}^{T}\right):\left(\nabla \mathbf{u}+\nabla \mathbf{u}^{T}\right)+\kappa(\alpha) \mathbf{u}^{2}\right] d \Omega
$$

where $\mu(\dot{\gamma})$ is the non-Newtonian viscosity.

The energy dissipation formulation for the turbulent case with Spalart-Allmaras model 
is given by:

$$
E_{T}(\alpha)=\int_{\Omega}\left[\frac{1}{2}\left(\mu+\mu_{T}\right)\left(\nabla \mathbf{u}+\nabla \mathbf{u}^{T}\right):\left(\nabla \mathbf{u}+\nabla \mathbf{u}^{T}\right)+\kappa(\alpha) \mathbf{u}^{2}\right] d \Omega
$$

where $\mu$ is the fluid molecular viscosity, and $\mu_{T}$ is the turbulent viscosity given by Eq. 3.12 .

Finally, the energy dissipation formulation for the non-Newtonian fluid coupled with the turbulent flow is given by:

$$
E_{n n_{T}}(\alpha)=\int_{\Omega}\left[\frac{1}{2}\left(\mu(\dot{\gamma})+\mu_{T}\right)\left(\nabla \mathbf{u}+\nabla \mathbf{u}^{T}\right):\left(\nabla \mathbf{u}+\nabla \mathbf{u}^{T}\right)+\kappa(\alpha) \mathbf{u}^{2}\right] d \Omega
$$

\section{The Relation with Shear Stress}

A critical criterion for designing devices for blood flow is to minimize blood damage. Since shear stress in the flow field is related to hemolysis (damage to red blood cells) and it also influences processes as platelet aggregation and thrombus formation (PROBST et al. 2010), it can be a good indicator of quality in a biomedical application.

A common criterion used in blood flow problems is wall shear stress, which is typically defined on the channel wall. During the optimization process, the interface between solid and fluid changes continuously, which makes it challenging to evaluate this parameter.

Topology optimization authors usually consider the shear rate as a representative measure of shear stress. Thus, we aim to minimize the integral of the squared shear rate as an objective function ABRAHAM; BEHR; HEINKENSCHLOSS, 2005):

$$
J_{s s}=\int_{\Omega} 2 \epsilon(\mathbf{u}): \epsilon(\mathbf{u}) d \Omega
$$

where $\epsilon(\mathbf{u})$ is the velocity shear rate defined in Eq. 2.5. Thus, it can be written as:

$$
J_{s s}=\int_{\Omega} 2\left(\nabla \mathbf{u}+\nabla \mathbf{u}^{T}\right):\left(\nabla \mathbf{u}+\nabla \mathbf{u}^{T}\right) d \Omega
$$

It is crucial to notice that the only differences between this objective function and the previously defined energy dissipation objective function are the absence of the dynamic viscosity term and the multiplying constant.

\subsubsection{Vorticity}

Rotating systems have a high tendency to vortex formations that causes a swirl motion on the fluid generating an undesirable vorticity current, implying on pressure loss and flow slip. Besides that, this reverse current can cause local cavitation (FRASER, 2001). 
Vorticity represents the shear stress in the fluid, and high vorticity can damage particles flowing in the current, causing a loss of material integrity, due to fluid mixing. It can be hazardous to sensitive fluids such as blood. Also, the literature indicates that minimizing the overall vorticity is a valid way to optimize the hematological conditions of a blood pump (ANTAKI et al., 1995, GHATTAS; HE; ANTAKI, 1995), given that it may reduce recirculation and hence thrombosis (ROMERO; N. Silva, 2017).

Considering the inertial reference system, the rotor rotation contributes to vortex formation, in axial direction, through Coriolis parameter, i.e., contributes with $\boldsymbol{f}=2 \omega$. Thus, the absolute vorticity $\eta$ in the inertial reference is equal to the sum of relative vorticity with rotor vorticity:

$$
\eta=\nabla \times \mathbf{u}+2 \omega
$$

In this work, only relative vorticity will be considered, and for a bi-dimensional case, the expression is given by (WHITE, 2010):

$$
\nabla \times \mathbf{u}=\left(\frac{\partial u_{2}}{\partial x_{1}}-\frac{\partial u_{1}}{\partial x_{2}}\right) e_{3}
$$

where $u_{1}$ and $u_{2}$ are relative velocity components on $x_{1}$ and $x_{2}$ direction, respectively.

A functional form for vorticity is expressed by (QUARTERONI; ROZZA, 2003; ABRAHAM; BEHR; HEINKENSCHLOSS, 2004):

$$
J(\mathbf{u})=\int_{\Omega}|\nabla \times \mathbf{u}|^{2} d \Omega=\int_{\Omega}\left(\frac{\partial u_{2}}{\partial x_{1}}-\frac{\partial u_{1}}{\partial x_{2}}\right)^{2} d \Omega
$$

It is shown in the results that the vorticity calculated in the relative form has a high correlation with the energy dissipation functional. Hence, even though it is not used as an objective function it is minimized indirectly by using the energy dissipation functional.

\subsubsection{Non-Newtonian TO Problem Formulation}

The problem to be solved for the non-Newtonian cases minimizing energy dissipation is given by:

$$
\begin{aligned}
\text { Minimize : } & E_{n n}(\alpha) \\
\alpha & \\
\text { subjected to }: & R_{m}=0 \\
& R_{c}=0 \\
& \int_{\Omega} \alpha d \Omega \leq f|\Omega| \\
& 0 \leq \alpha \leq 1
\end{aligned}
$$


where $R_{m}$ is the non-newtonian momentum equation weak form (Eq. 4.2) and $R_{c}$ is the continuity equation (Eq. 4.1).

\subsubsection{Turbulent TO Problem Formulation}

The problem to be solved for the cases minimizing energy dissipation and the Spalart-Allmaras turbulence model is given by:

$$
\begin{aligned}
\text { Minimize : } & E_{T}(\alpha) \\
\alpha & \\
\text { subjected to }: & R_{R A N S m}=0 \\
& R_{R A N S c}=0 \\
& R_{S A}=0 \\
& R_{e i k}=0 \\
& \int_{\Omega} \alpha d \Omega \leq f|\Omega| \\
& 0 \leq \alpha \leq 1
\end{aligned}
$$

where $R_{R A N S m}$ is the RANS momentum equation weak form (Eq. 4.5), $R_{R A N S c}$ is the continuity equation weak form (Eq. 4.4), $R_{S A}$ is the Spalart-Allmaras equation weak form (Eq. 4.6), and $R_{e i k}$ is the Eikonal equation weak form (Eq. 4.7).

\subsubsection{Non-Newtonian Turbulent TO Problem Formulation}

The problem to be solved for the cases minimizing energy dissipation and the Carreu-Yasuda non-Newtonian coupled with the Spalart-Allmaras turbulence model is given by:

$$
\begin{aligned}
\text { Minimize : } & E_{n n_{T}}(\alpha) \\
\alpha & \\
\text { subjected to : } & R_{n n_{R A N S m}}=0 \\
& R_{R A N S c}=0 \\
& R_{S A}=0 \\
& R_{e i k}=0 \\
& \int_{\Omega} \alpha d \Omega \leq f|\Omega| \\
& 0 \leq \alpha \leq 1
\end{aligned}
$$

where $R_{R A N S c}$ is the continuity equation weak form (Eq.4.4), $R_{S A}$ is the Spalart-Allmaras equation weak form (Eq. 4.6), $R_{e i k}$ is the Eikonal equation weak form (Eq. 4.7), and $R_{n n_{R A N S m}}$ is the RANS momentum equation weak form (Eq. 4.5 however considering the fluid molecular viscosity $(\mu)$ as the Carreau-Yasuda non-Newtonian viscosity model (Eq. 2.6 . 


\section{SENSITIVITY OF THE TOPOLOGY OPTIMIZATION PROBLEM}

This chapter describes the sensitivity calculation for all the cases defined in Chapters 2 and 5 with respect to the design variable. The sensitivities are used to perform the optimization, as shown in Chapter 7.

\subsection{Adjoint Method}

The gradients with respect to the design variables are required for the optimization algorithm to define the topology. In this work, the adjoint approach is used. The adjoint method creates a problem given by the derivation of the governing equation in the weak form and allows obtaining the functional gradient by using the derivatives with respect to the output variables.

\subsubsection{Continuous Adjoint Approach}

Defining a cost functional $J$ to be minimized under the constraint $R(\mathbf{u}, p, \alpha)=0$, which is composed of the state equations, the following optimization problem can be stated:

$$
\text { minimize } J(\mathbf{u}, p, \alpha) \quad \text { subject to } R(\mathbf{u}, p, \alpha)=0
$$

where $\mathbf{u}$ is the velocity field, $p$ is the pressure field and $\alpha$ is the design variable. In this case, the constraint $R$ are the Navier-Stokes equations coupled with the continuity equation.

Then, a Lagrangian problem can be stated as:

$$
L:=J-\int_{\Omega}(\mathbf{a}, b) R d \Omega
$$

where $\mathbf{a}$ and $b$ are the Lagrange multipliers for the velocity and pressure, respectively. These Lagrange multipliers are the adjoint variables for each field.

In order to calculate the sensitivities of the desired functional it is necessary to compute the total variation of $L$ OTHMER, 2008):

$$
\delta L=\delta_{\alpha} L+\delta_{\mathbf{u}} L+\delta_{p} L
$$

Since changes in $\alpha$ causes variations in the flow field, the total variation of $L$ also includes contributions from the changes in $\mathbf{u}$ and p. Hence, computing the sensitivities 
directly from Eq. 6.3 would require to solve the state equations once for each design variable. However, for the adjoint method, we can choose Lagrange multipliers a and $b$ such that the variation with respect to the state variables vanishes identically:

$$
\delta_{\mathbf{u}} L+\delta_{p} L=0
$$

this equation is the foundation to the definition of the adjoint problem, which is shown in Appendix A.

Then, the sensitivities can be computed by (OTHMER, 2008):

$$
\delta L=\delta_{\alpha} L=\delta_{\alpha} J-\int_{\Omega}(\mathbf{a}, b) \delta_{\alpha} R d \Omega
$$

Thus, if we consider the energy dissipation functional (Eq. 5.9) and a Newtonian Navier-Stokes flow, the sensitivity is:

$$
\begin{aligned}
\frac{\partial L}{\partial \alpha} & =\frac{\partial J}{\partial \alpha}-\int_{\Omega}(\mathbf{a}, b) \frac{\partial R}{\partial \alpha} d \Omega \\
& =(\mathbf{u} \cdot \mathbf{u}-\mathbf{a} \cdot \mathbf{u}) \frac{\partial \kappa(\alpha)}{\partial \alpha}
\end{aligned}
$$

where, $\mathbf{u}$ is the velocity field result from the state equations, $\mathbf{a}$ is the adjoint velocity field result from the adjoint equations (Eq. A.9p, and $\frac{\partial \kappa(\alpha)}{\partial \alpha}$ is given by:

$$
\frac{\partial \kappa(\alpha)}{\partial \alpha}=\left(\kappa_{\min }-\kappa_{\max }\right)(1+q) \frac{q}{(\alpha+q)^{2}}
$$

In order to compute the sensitivities for different cost functionals, the same procedure can be employed.

\section{The non-Newtonian case}

In the non-Newtonian fluid model, there are additional terms that arise from the viscosity dependence on both the velocity gradient and the design variable. Thus, the problem becomes:

$$
\text { minimize } J_{n n}(\mathbf{u}, p, \alpha, \mu(\mathbf{u}, \alpha)) \quad \text { subject to } R_{n n}(\mathbf{u}, p, \alpha, \mu(\mathbf{u}, \alpha))=0
$$

Hence, the Lagrangian is given by

$$
L_{n n}:=J_{n n}-\int_{\Omega}(\mathbf{a}, b) R_{n n} d \Omega
$$

and its variation by

$$
\delta L_{n n}=\delta_{\alpha} L_{n n}+\delta_{\mathbf{u}} L_{n n}+\delta_{p} L_{n n}
$$


The variation $\delta_{u} L_{n n}$ has a non-linear dependence on the velocity due to the Carreau-Yasuda viscosity $\mu(\mathbf{u}, \alpha)$ (Eq. 2.6). Thus, the derivation of the continuous adjoint formulation is difficult. However, with the use of automatic derivation algorithms (FUNKE; FARRELL, 2013; DILGEN et al., 2018a) it can be calculated more easily. In this work, this approach is used and is described in the numerical implementation chapter (Chp. 7).

\section{The Spalart-Allmaras turbulent case}

The addition of the turbulence model increases the number of equations to be solved. This causes the number of adjoint equation to increase as well, given that two equation are added to the forward problem. The minimization problem is given by:

$$
\begin{aligned}
\text { minimize : } & J_{S A}\left(\mathbf{u}, p, \alpha, \mu\left(\nu_{t}, \alpha\right)\right) \\
\text { subjected to : } & R_{R A N S}\left(\mathbf{u}, p, \alpha, \mu\left(\nu_{t}, \alpha\right)\right)=0 \\
& R_{S A}\left(\nu_{t}, \mathbf{u}, \phi, \alpha\right)=0 \\
& R_{e i k}(\phi, \alpha)=0
\end{aligned}
$$

The Lagrangian is given by

$$
L_{S A}:=J_{S A}-\int_{\Omega}(\mathbf{a}, b) R_{R A N S} d \Omega-\int_{\Omega} c R_{S A} d \Omega-\int_{\Omega} d R_{e i k} d \Omega,
$$

where $\mathbf{a}$ is the adjoint velocity, $b$ is the adjoint pressure, $c$ is the adjoint eddy viscosity, and $d$ is the adjoint wall distance.

The total variation of $L_{S A}$ is

$$
\delta L_{S A}=\delta_{\alpha} L_{S A}+\delta_{\mathbf{u}} L_{S A}+\delta_{p} L_{S A}+\delta_{\nu_{t}} L_{S A}+\delta_{\phi} L_{S A}
$$

The adjoint problem is defined by zeroing the variation with respect to the field variables, i.e.,

$$
\delta_{\mathbf{u}} L_{S A}+\delta_{p} L_{S A}+\delta_{\nu_{t}} L_{S A}+\delta_{\phi} L_{S A}=0
$$

The complete derivation is shown in the work Papoutsis-Kiachagias and Giannakoglou (2016). However, when the rotational frame and the non-Newtonian model are introduced coupled to the turbulence model, the derivation becomes more complex due to the non-linearity of the viscosity. Hence, in this work, the automatic derivation algorithm (FUNKE; FARRELL, 2013, DILGEN et al., 2018a) is used. This approach is described in the following chapter (Chp. 7). 


\section{NUMERICAL IMPLEMENTATION}

\subsection{Optimization Algorithm}

The optimization algorithm can be a stochastic algorithm, which generates and uses random variables, such as genetic algorithms; or gradient-based, where the gradient information with respect to the desired functional is evaluated and gives the direction to update the design variables values. In this work, a gradient-based algorithm will be used.

The topology optimization procedure is described in Section 1.3 , it consists mainly of FEM resolution, gradient evaluation, and optimization algorithm. The process is iterative and consists of the following steps:

(a) Solve the governing equations with given initial value for design variables;

(b) Solve adjoint equations based on velocity and pressure fields of (a);

(c) Calculate the sensitivities of objective functions and constraints;

(d) Update the design variable value with an optimization algorithm;

(e) Verify the stopping criteria.

The stopping criterion usually is defined as a norm between two consecutive iterations, as

$$
\left\|\alpha^{k}-\alpha^{k-1}\right\|_{\infty} \ll 1 \times 10^{-3}
$$

where $\alpha$ is the design variable distribution and $k$ is the iteration number.

\subsection{Software Environment}

By using the equations presented in the previous sections, it is possible to implement a software to solve the partial differential equations (PDE). There are different software packages that are capable of solving the PDEs by the Finite Element Method (FEM), some require a manual implementation, such as the commercial environment MATLAB $\AA$, others have modules that receive the weak problem formulation and assemble the FEM problem, such as the commercial software COMSOL and the Open Source software FEniCS. In this work, the FEniCS environment and some external libraries, such as the pyadjoint and the pyIPOPT, will be used. A more detailed explanation of these libraries is presented in the following sections. 


\subsubsection{Solving Differential Equations - The FEniCS system}

The FEniCS system (LOGG; WELLS; BOOK, 2012) is a collection of software components for automating the solution of PDEs by the finite element method. This involves translation of a mathematical syntax to a language that can be interpreted by the computer. So, aiming to use the weak formulation as a software input, it is necessary to use a software capable of interpreting this high-level language and transforming it into a numerical routine. This interpretation software is usually referred to as Form Compiler $(\mathrm{FC})$.

The FEniCS environment has its own form compiler, the FEniCS Form Compiler (FFC), that receives a discrete form of the weak variational equation given in Unified Form Language (UFL) (ALNÆS et al., 2014), similar to the mathematical formulation, and generates a $\mathrm{C}++$ code of the finite element assembly in the format of the Unified Form-Assembly Code (UFC) (ALNAES et al. 2009). This assembly is an optimized low-level code that evaluates the local element tensors.

The local tensors are used by DOLFIN (LOGG; WELLS, 2010), a library that handles the communication between all FEniCS modules. This library also provides various data structures to interface meshes, function spaces, functions, and solvers. These tensors are coded in a format of SciPy, a Python-based Open Source software that has modules to handle scientific computing. This enables the use of many linear algebra packages, such as PETSc, Epetra, and uBLAS, which permits to select among many linear solvers and preconditioners.

\subsubsection{Solvers and Preconditioners}

The FEM equations, in general, large sparse systems of linear equations, are solved by iterative methods. Many methods exist for solving this type of problems, and it is necessary to find one that is effective and reliable. Unfortunately, an approach that works well for one problem type might not work as well for another, and sometimes it does not even converge.

The Navier-Stokes in the rotating reference system and the Spalart-Allmaras equations are problems that hardly converges when the basic Newton method is used. Thus, more complex nonlinear solvers are needed to guarantee the solution convergence. Hence, a more complex solver is used, defined as "Reduced space active set solvers for variational inequalities based on Newton's method" described in Benson and Munson (2006) associated with a secant line search method. This solver is present in the PETSc package (BALAY et al., 2018).

The FEniCS system offers pre-installed support to linear solvers such as Generalized minimal residual (Gmres) and Biconjugate gradient stabilized (Bicstab), that are usually 
used on linearized Navier-Stokes problems. However, some external solvers can be used, and in this work, the MUltifrontal Massively Parallel Sparse direct solver (MUMPS) (AMESTOY et al., 2001) is used.

\subsubsection{Solution of the Adjoint Problem}

There are some methods to derive the adjoint model from the PDE. The first and more intuitive is to do the same steps of the manual manipulation of the forward equations. Some software tries to mimic this by automating this by-hand manipulation, such as the SAGE (STEIN; JOYNER, 2005). A second method is to derive at a source code level, treating the forward model as a sequence of elementary instructions (FARRELL et al., 2013). A third method consists of doing the derivation of the forward method at a middle-level approach, that can be automated without depending on low-level implementation details. These three approaches are presented in Figure 7.1, summarizing the possible ways to derive the adjoint model starting from the forward equations, under the FEniCS environment.

Figure 7.1 - Methods for obtaining the adjoint code.

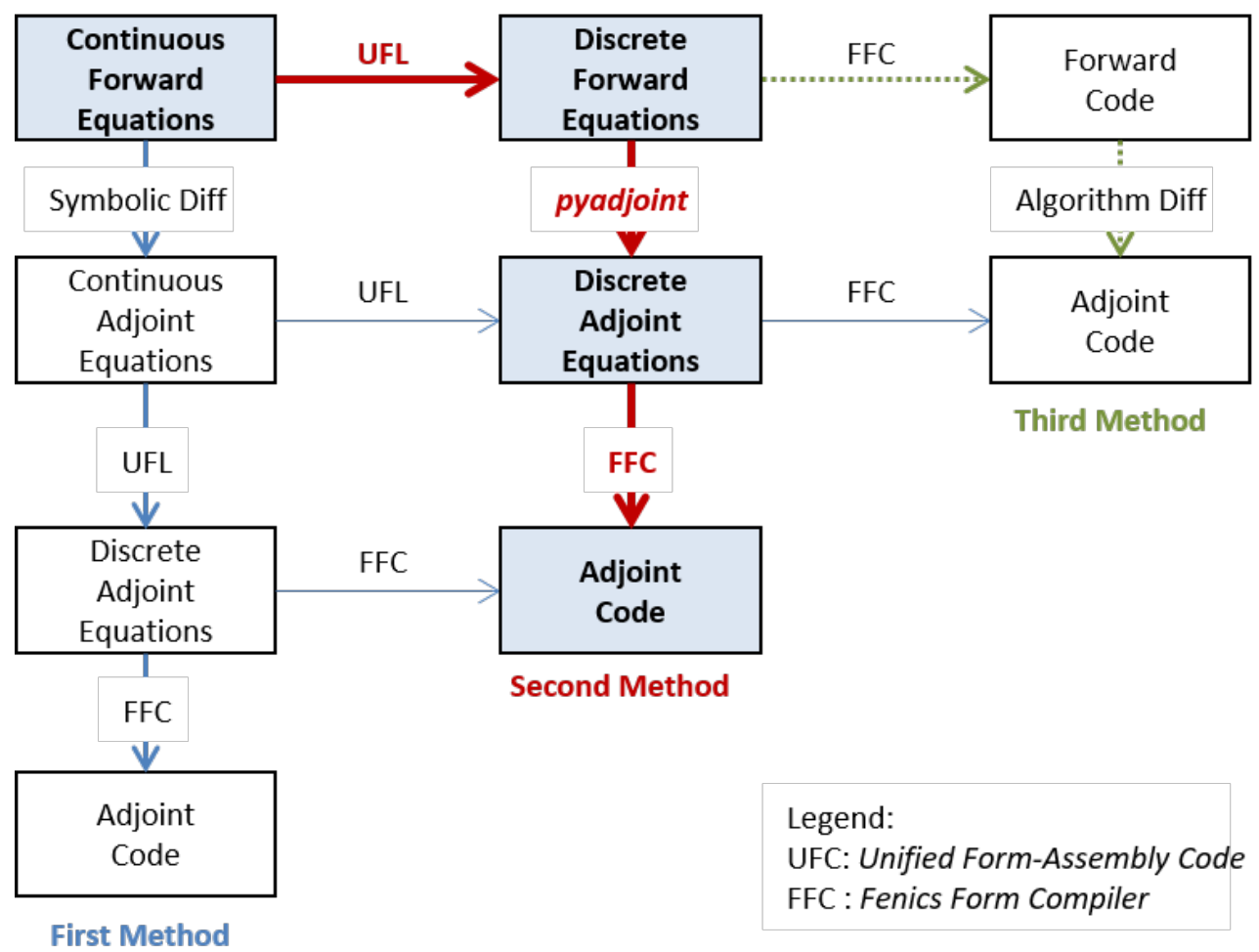

Adapted from Farrell et al. (2013)

The second method relies on the library pyadjoint, that enables the automatic derivation and solution of tangent linear and adjoint models. The purpose of pyadjoint is to facilitate the development of these models based on the fundamental abstraction of considering the forward model as a sequence of equation solutions. Based on this abstraction, the library builds a symbolic description of the forward model, from which it can automatically derive the symbolic representation of the associated tangent linear and adjoint systems (FUNKE; 
FARRELL, 2013).

In this work, the path in bold in Figure 7.1 (Second Method) is used. Thus, the forward equations are discretized by using the UFL package, then the discrete adjoint equations are derived by using the pyadjoint library, and for last the adjoint code is assembled through the FFC package. Also, in order to interface DOLFIN with pyadjoint there is the dolfin-adjoint software, that gets the adjoint model derived by the pyadjoint and translates it to the same high-level language of the forward model, i.e., it makes the connection between UFL and FFC.

\subsubsection{Solution of the Optimization Problem}

In this work, the topology optimization problem is implemented by using the framework formed by the FEniCS system, to solve the PDEs, the pyadjoint library, to compute the adjoint model, and an optimizer, to update the domain. These software are coded in Python language, so, to simplify the implementation, the optimizer also has to be coded in this language. A series of optimizers offer compatibility with Python and FEniCS, such as the pyOpt package, that has some well-known optimizers as the MMA (SVANBERG, 1987) and the ALGENCAN. However, in this work another package is used, the IPOpt (WÄCHTER, 2002, WÄCHTER; BIEGLER, 2006, WÄCHTER, 2009), which implements the Internal Point Optimization algorithm.

The IPOpt software implements a primal-dual barrier method that solves a sequence of barrier problems. The complete algorithm can be found in (WÄCHTER, 2002), however, the approach for the barrier method is briefly described as follows. The generic minimization problem is given by Eq. 7.2 .

$$
\begin{aligned}
\text { Minimize : } & f(x) \\
x \in \mathbb{R}^{n} & \\
\text { subjected to : } & c(x)=0 \\
& x^{(i)} \geq 0 \quad \text { for } i \in I
\end{aligned}
$$

The barrier method replaces the bound constraints by a logarithmic barrier term which is added to the objective function giving:

$$
\begin{aligned}
\text { Minimize }: & \phi_{\mu}(x):=f(x)-\mu \sum_{i \in I} \ln \left(x^{(i)}\right) \\
x \in \mathbb{R}^{n} & \\
\text { subjected to }: & c(x)=0
\end{aligned}
$$

where $\mu>0$ is the barrier parameter, $\phi_{\mu}(x)$ is the barrier function and $-\mu \sum_{i \in I} \ln \left(x^{(i)}\right)$ is the barrier parameter. 
The degree of influence of the barrier term is determined by the size of $\mu$, and the barrier problem solution eventually converges to the solution of the original problem as $\mu \rightarrow 0$. Thus, an initial value for the barrier parameter is defined and the barrier problem is solved, then the barrier parameter is updated and the loop restarts. The loop is terminated when a tolerance for $\mu_{l+1}-\mu_{l}$ is reached.

Figure 7.2 - Topology optimization implementation flow chart.

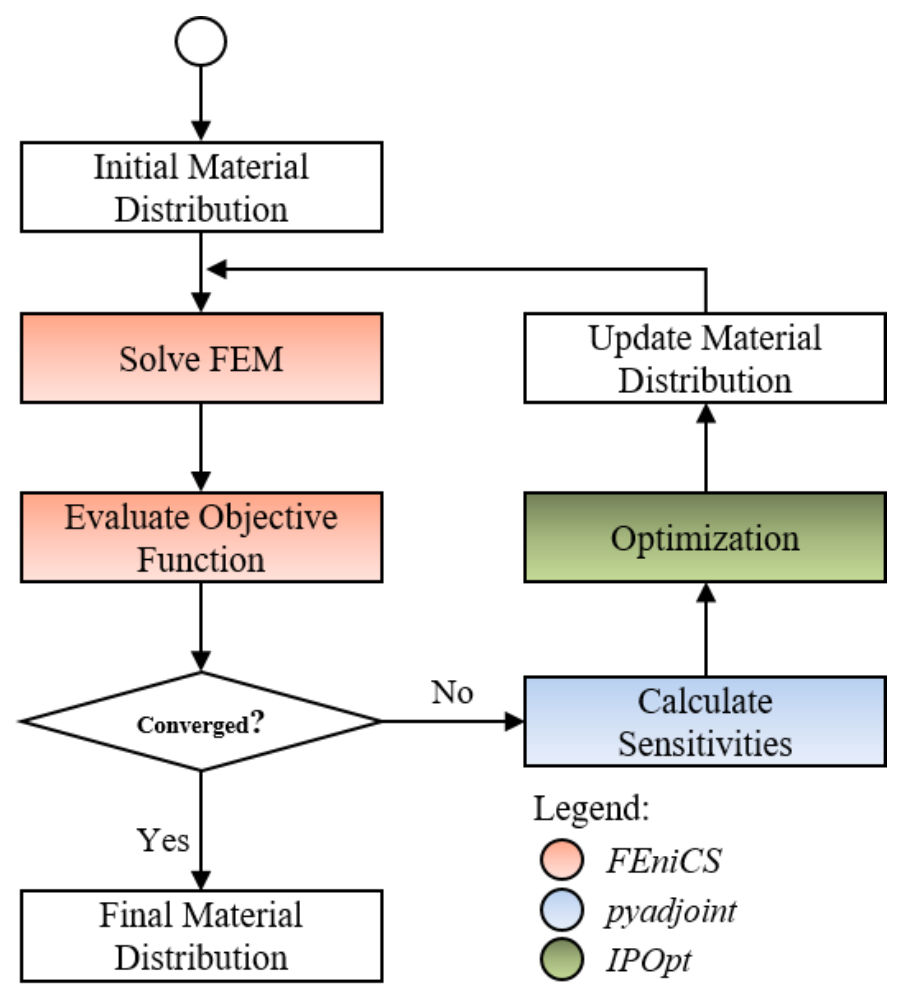

\subsubsection{Implementation of Flow Machine Optimization}

The sequence of steps to solve the optimization involves firstly defining an initial material distribution and the boundary conditions, and then the FEniCS routines are called to solve the FEM system returning the solution vector $\left[\mathbf{u}_{1} \mathbf{u}_{2} \mathbf{p}\right]$. Specifically for the turbulence problem, it is difficult to solve all equations coupled, so a step iteration is performed by solving first the RANS Eq. and then the Spalart-Allmaras Eq. until convergence is reached, as shown in Fig. 7.3. 
Figure 7.3 - Iteration Step to solve the forward problem in the turbulent case.

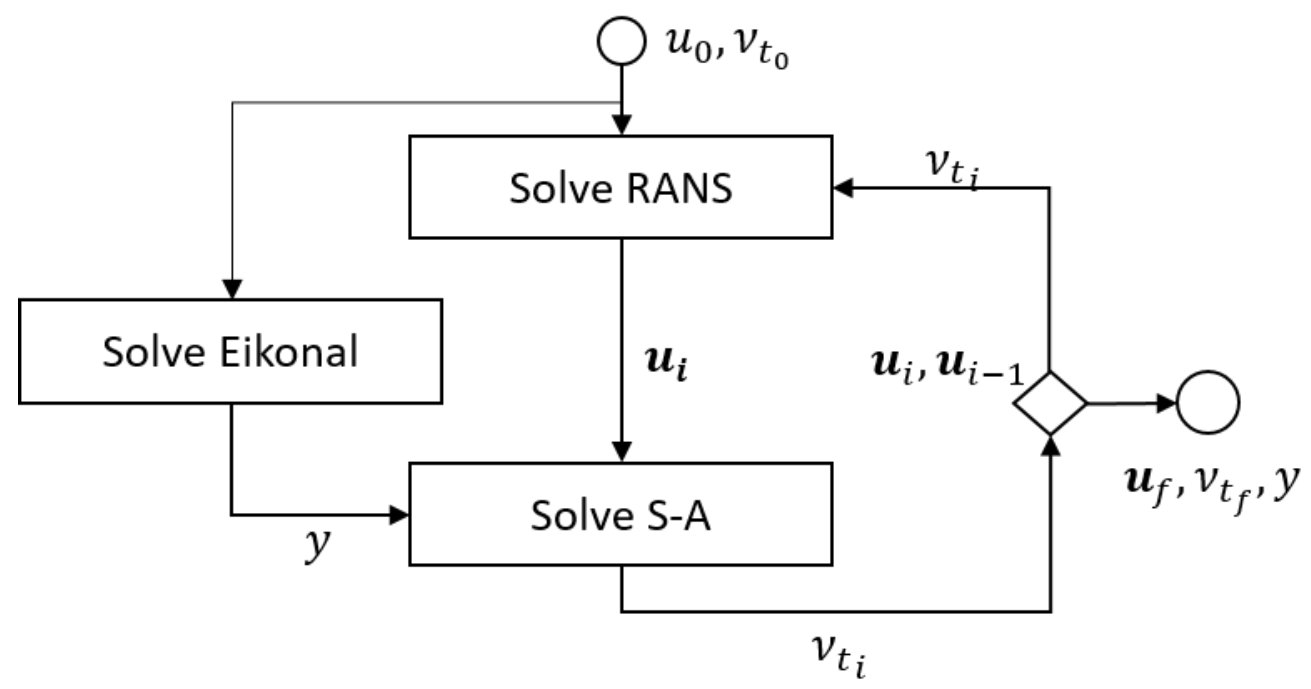

With this, the functional is evaluated, and the dolfin-adjoint is called and performs the adjoint problem derivation, in order to calculate the sensitivity of the functional with respect to the design variable. Next, this information is passed to the optimizer IPOpt, that computes the next material distribution over the domain. The process is repeated until the functional value converges, in which case the optimum solution is reached. Figure 7.2 shows the steps cited above.

The FEniCS has a user-friendly interface, as cited above, where the implementation of the weak form problem can be done in a language similar to the math syntax. Thus, the implementation of Navier-Stokes in a rotating reference system equations can be done as shown in Listing 7.1 .

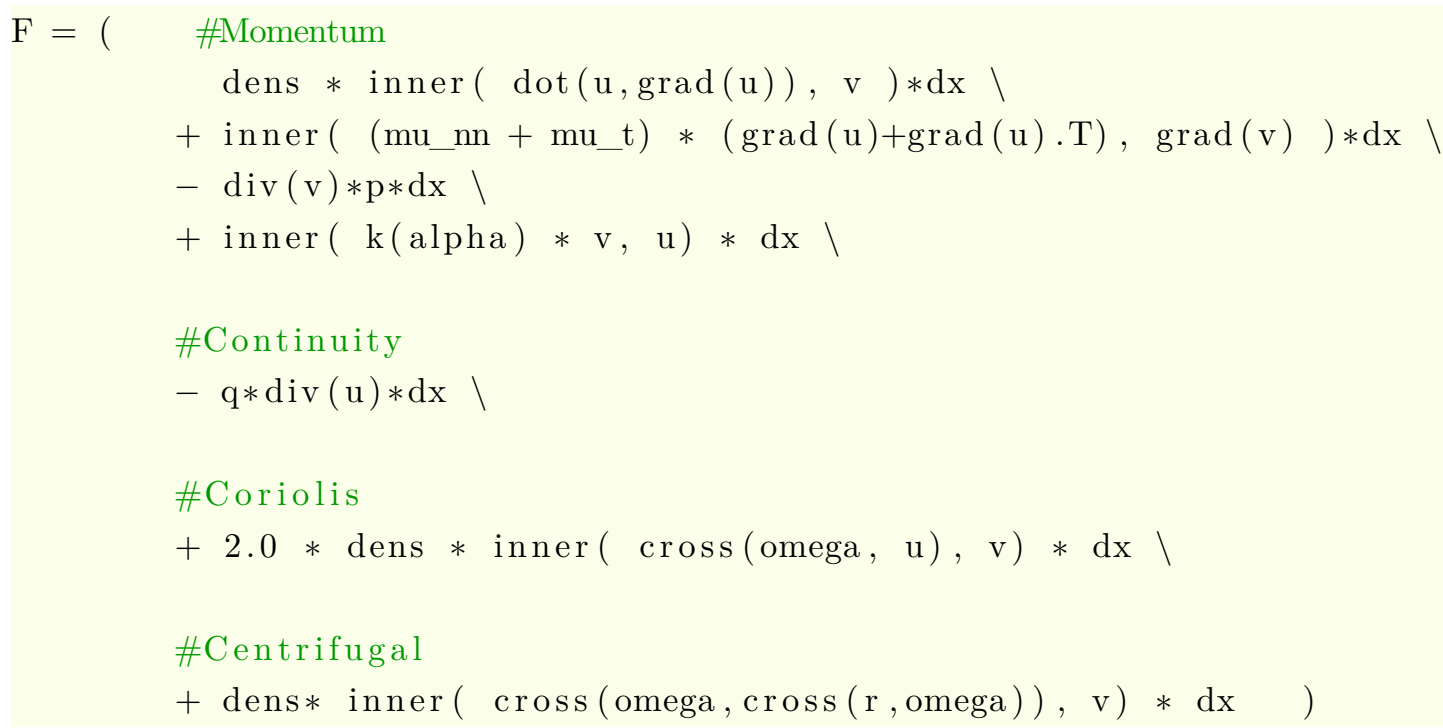

Listing 7.1 - Variational problem in FEniCS notation. 


\section{NUMERICAL RESULTS}

This chapter shows the topology optimization results for impeller and volute considering the non-Newtonian model and the Spalart-Allmaras turbulence model. First some impellers design considering arbitrary Newtonian fluid properties are shown in order to evaluate the turbulence algorithm behavior. Then, impeller and volute results considering blood, real properties, and both laminar and turbulent models are shown.

The impeller domain is interpreted by selecting a sector from a reference impeller and abstracting the selection as a 2D domain, as shown in Fig. 8.1.

Figure 8.1 - Impeller domain interpretation.
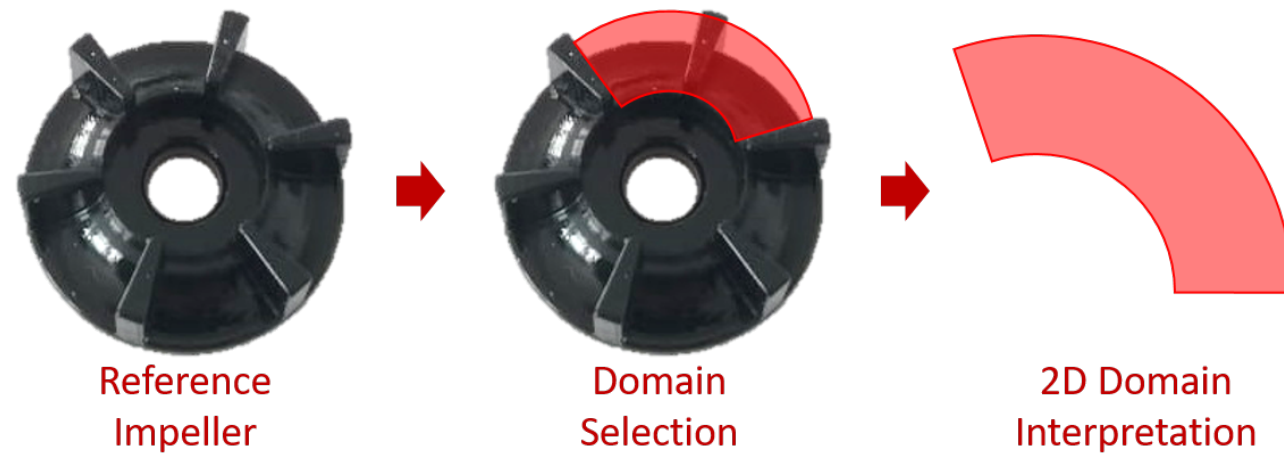

The meshes used in the results are triangular unstructured meshes with approximately 57,000 elements. The impeller mesh is shown in Fig. 8.2.

Figure 8.2 - Impeller mesh.

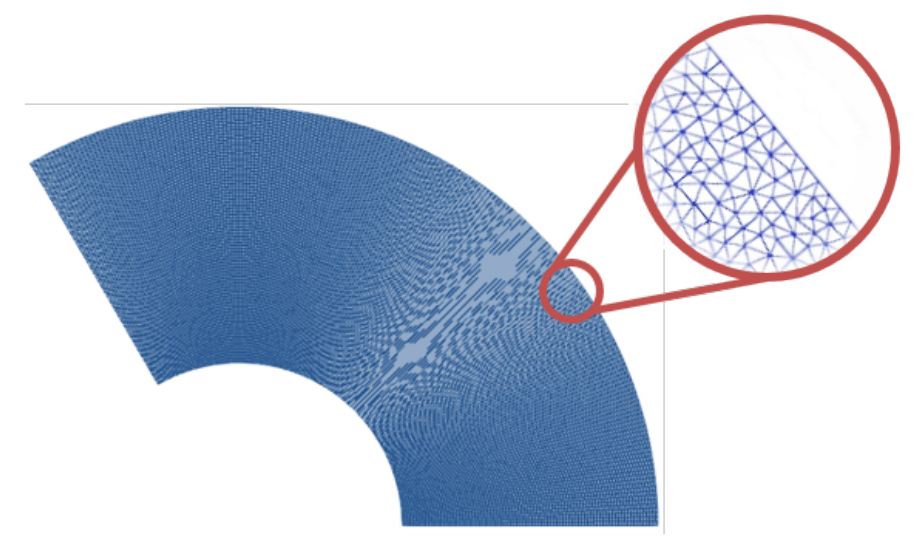

\subsection{Impeller Design (Turbulent)}

This section presents the model and results for the rotor design considering the Newtonian fluid (with arbitrary properties) operating at high Reynolds numbers with 
the turbulence model of Spalart-Allmaras. This study is done to explore the algorithm behavior under different flow conditions.

The verification study of the finite element model implemented can be seen in Appendix B.3.

First, a case with low Reynolds numbers is studied by comparing the optimized topologies for Energy Dissipation (Eq. 5.9) obtained with the Navier-Stokes (NS) model and with RANS-SA (RANS with Spalart-Allmaras turbulence model). Then the same analysis is performed for high Reynolds numbers. Finally, a cross-check is performed by solving the forward problem with both models in both topologies.

\subsubsection{Domain and Boundary Conditions}

The domain dimensions are inner radius of $4[\mathrm{~mm}]$, outer radius $10[\mathrm{~mm}]$ and $180^{\circ}$ circular section, and the boundary conditions are shown in Fig 8.3 . For the Navier-Stokes problem, the domain outlet has a zero tension condition, the walls have a no-slip condition, and the inlet has a parabolic velocity profile normal to the boundary. Two inlet velocities and rotations are considered in order to analyze the effect of the Reynolds number in the results. The Spalart-Allmaras problem has a zero viscosity variation in the normal direction at the outlet, zero viscosity at the walls and a prescribed viscosity at the inlet.

Figure 8.3 - Boundary conditions for the turbulent rotor optimization with arbitrary dimensions.

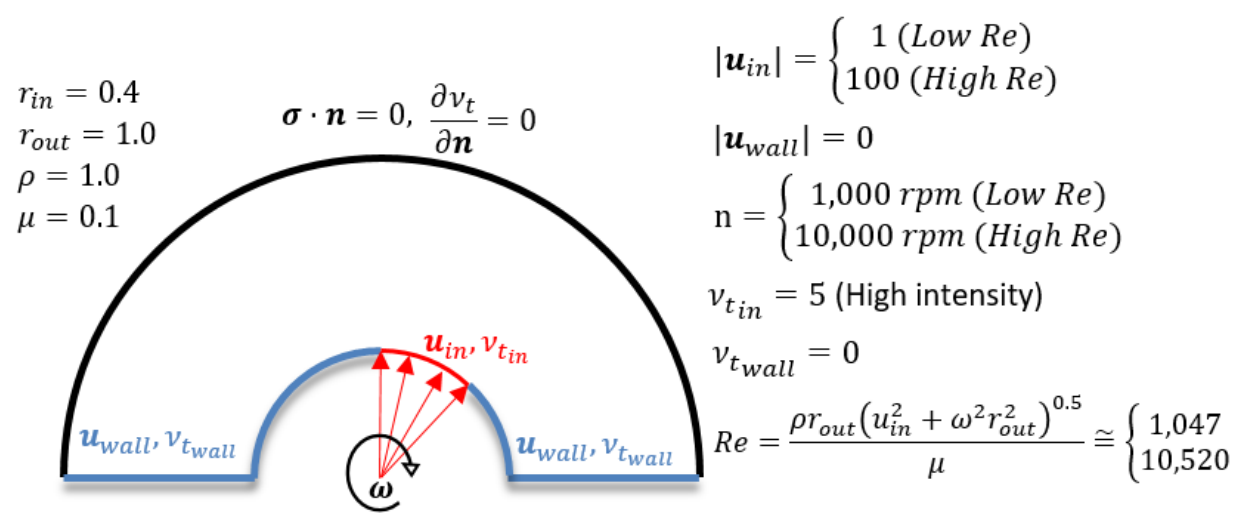

\subsubsection{Low Reynolds Optimization}

This section presents the optimized topologies obtained with NS and RANS-SA for an inlet velocity of 1.0, rotation of 1,000 [rpm], and Reynolds of 1,047. The initial guess considered is the curved blade shown in Fig. 8.4. Table 8.1 shows the constants and boundary condition inputs for this case. 
Figure 8.4 - Initial guess: Curved Blade.

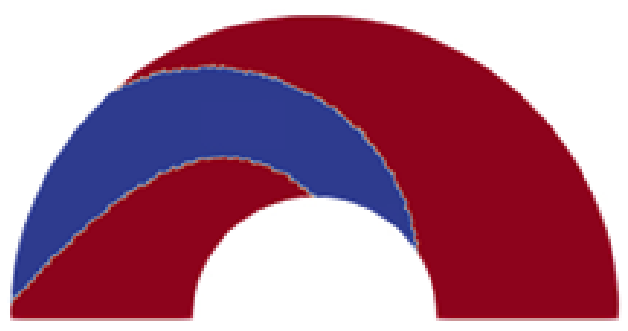

Table 8.1 - Inputs for Low Reynolds Turbulent Optimization.

\begin{tabular}{lcc}
\hline Parameter & NS & RANS-SA \\
\hline$r_{\text {in }}$ & 0.4 & 0.4 \\
$r_{\text {out }}$ & 1.0 & 0.4 \\
$\rho$ & 1.0 & 1.0 \\
$\mu$ & 0.1 & 0.1 \\
$\left|u_{\text {in }}\right|$ & 1.0 & 1.0 \\
$\left|u_{\text {wall }}\right|$ & 0.0 & 0.0 \\
$\omega$ & 167.0 & 167.0 \\
$\nu_{t_{\text {in }}}$ & 5.0 & 5.0 \\
$\nu_{t_{\text {wall }}}$ & 0.0 & 0.0 \\
$k_{\text {max }}$ & 10,000 & 10,000 \\
$q$ & $0.01-0.1$ & 10.0 \\
Volume Constraint & $30 \%$ & $30 \%$ \\
Initial Distribution & Curve Blade & Curved Blade \\
\hline
\end{tabular}

The optimized topologies for NS and RANS-SA are shown in Figs. 8.5a and 8.5b, respectively. These results are obtained with $k_{\max }=10^{4}$, different values of $q$ (Eq. 5.1) and a volume restriction of $25 \%$ of the total area. The topologies are very different indicating that the process probably stagnated at a local minimum for each case.

Figure 8.5 - Optimized topologies for Low Reynolds Case.

(a) Navier-Stokes.

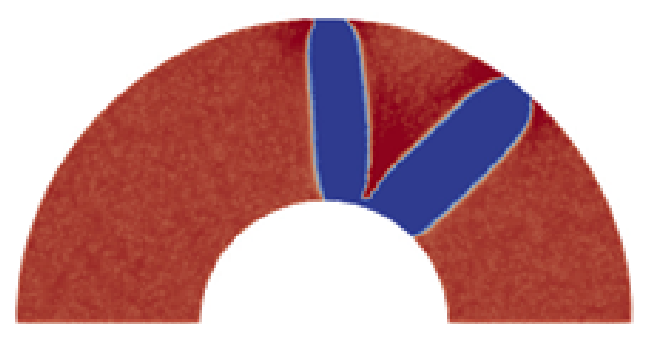

(b) RANS-SA.

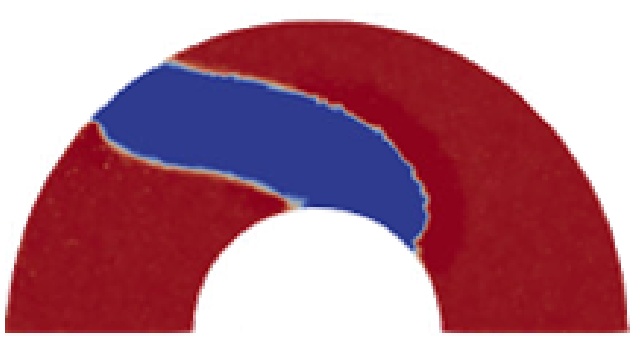

Table 8.2 shows the functional value for each topology calculated with the Navier-Stokes equations. This is done to have a base of comparison between the topologies. Further analysis with both models is shown in Sec. 8.1.4. The \% column represents the percentage 
Table 8.2 - Objective function values for Low Reynolds Case calculated with NS model.

\begin{tabular}{|c|c|c|c|c|c|}
\hline Case & $\begin{array}{c}\text { Initial guess } \\
\text { Fig. } 8.4\end{array}$ & $\begin{array}{c}\text { N-S } \\
\text { Fig. } 8.5 \mathrm{a}\end{array}$ & $\%$ & $\begin{array}{l}\text { RANS-SA } \\
\text { Fig. } 8.5 \mathrm{~b}\end{array}$ & $\%$ \\
\hline Area & 0.38 & 0.33 & $-14 \%$ & 0.33 & $-14 \%$ \\
\hline Mass Flow & 0.30 & 0.30 & $0 \%$ & 0.30 & $0 \%$ \\
\hline Energy D. (Eq. 5.9$)$ & 14.03 & 10.05 & $-28 \%$ & 10.73 & $-24 \%$ \\
\hline Viscous D. (Eq. 5.10 & 13.30 & 8.89 & $-33 \%$ & 9.72 & $-27 \%$ \\
\hline Friction D. (Eq. 5.11 ) & 0.73 & 1.16 & $60 \%$ & 1.00 & $37 \%$ \\
\hline
\end{tabular}

change in relation to the initial guess value. As can be seen, both optimized blades present a better energy dissipation value than the initial guess. It is important to notice that the final goal of the optimization is to obtain a better value for the viscous dissipation. Thus, it is important to separate the functional value in two parts (Viscous and Friction Dissipation) for the analysis. In this case, the improvement in the energy dissipation seems to reflect an improvement in the viscous dissipation and indicates that for Low Reynolds numbers the NS model should be used.

Additional analysis is performed by post-processing the result by isolating the fluid region and smoothing the contour to verify the topologies without the influence of the material model. The post-processed results are shown in Fig. 8.6.

Figure 8.6 - Post-processed topologies for Low Reynolds Case calculated with NS model.

(a) Initial guess.

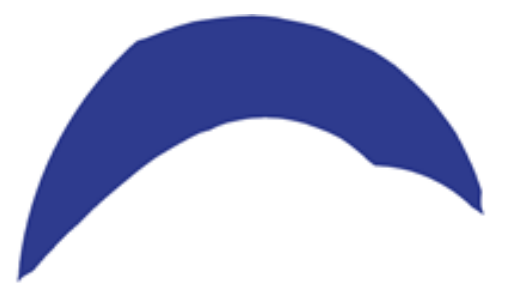

(b) Navier-Stokes.

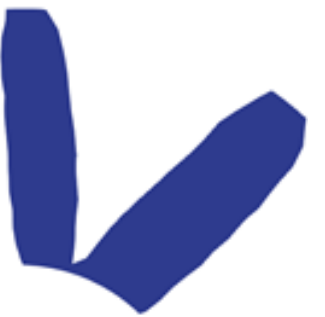

(c) RANS-SA.

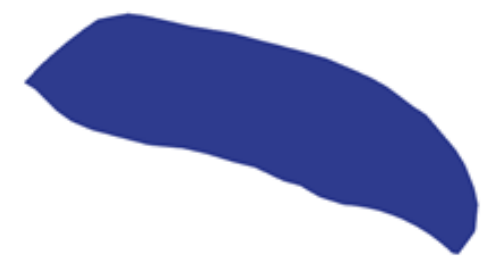

Table 8.3 shows the functional value for the post-processed topologies. The Friction Dissipation value, in this case, is zero because the material model is not present. It can be seen that both optimized blades still maintain the better value for the energy dissipation than the initial guess. However, here, the RANS-SA case has the lowest value, this could be associated to the post-processing procedure that modifies the original contour and removes the gray areas, which, by the way, reduced the calculated area. This indicates that for the NS with the material model (Fig. 8.5a), the gray areas help with the reduction of the viscous dissipation. Finally, for Low Reynolds numbers, both models could be used to achieve a better topology. 
Table 8.3 - Objective function values for Post-processed Low Reynolds Case.

\begin{tabular}{|c|c|c|c|c|c|}
\hline Case & $\begin{array}{c}\text { Initial guess } \\
\text { Fig. } 8.6 \mathrm{a}\end{array}$ & $\begin{array}{c}\text { N-S } \\
\text { Fig. } 8.6 \mathrm{~b}\end{array}$ & $\%$ & $\begin{array}{l}\text { RANS-SA } \\
\text { Fig. } 8.6 \mathrm{c}\end{array}$ & $\%$ \\
\hline Area & 0.37 & 0.21 & $-44 \%$ & 0.28 & $-23 \%$ \\
\hline Mass Flow & 0.30 & 0.30 & $0 \%$ & 0.29 & $-3 \%$ \\
\hline Energy D. (Eq. 5.9 & 14.24 & 10.76 & $-24 \%$ & 9.84 & $-31 \%$ \\
\hline Viscous D. (Eq. 5.10 & 14.24 & 10.76 & $-24 \%$ & 9.84 & $-31 \%$ \\
\hline Friction D. (Eq. 5.11$)$ & 0.00 & 0.00 & & 0.00 & \\
\hline
\end{tabular}

\subsubsection{High Reynolds Optimization}

This section presents the optimized topologies obtained with NS and RANS-SA for an inlet velocity of 100.0, rotation of 10,000 [rpm], and Reynolds of 10,520. The initial guess considered is the curved blade shown in the previous section in Fig. 8.4. Table 8.4 shows the constants and boundary condition inputs for this case.

Table 8.4 - Inputs for High Reynolds Turbulent Optimization.

\begin{tabular}{lcc}
\hline Parameter & NS & RANS-SA \\
\hline$r_{\text {in }}$ & 0.4 & 0.4 \\
$r_{\text {out }}$ & 1.0 & 0.4 \\
$\rho$ & 1.0 & 1.0 \\
$\mu$ & 0.1 & 0.1 \\
$\left|u_{\text {in }}\right|$ & 100.0 & 100.0 \\
$\left|u_{\text {wall }}\right|$ & 0.0 & 0.0 \\
$\omega$ & 1670.0 & 1670.0 \\
$\nu_{t_{\text {in }}}$ & 5.0 & 5.0 \\
$\nu_{t_{\text {wall }}}$ & 0.0 & 0.0 \\
$k_{\text {max }}$ & 100,000 & 100,000 \\
$q$ & 0.1 & 0.1 \\
Volume Constraint & $30 \%$ & $30 \%$ \\
Initial Distribution & Curve Blade & Curved Blade \\
\hline
\end{tabular}

The optimized topologies for the case with high Reynolds number are shown in Figs. $8.7 \mathrm{a}$ and $8.7 \mathrm{~b}$, respectively. These results are obtained with $k_{\max }=10^{5}$, different values of $q$ (Eq. 5.1) and a volume restriction of $25 \%$ of the total area. Differently from the low Reynolds case the topologies here are more similar.

The RANS-SA model creates a smoother curvature near the inlet; this is caused by the increased viscosity resulting from the turbulence model. From the physics point of view, this additional viscosity makes it harder to change the flow direction abruptly, so a slower direction change is more efficient. The same conclusion can be obtained from the objective function analysis, given that the eddy viscosity increases the relative weight 
from the viscous dissipation term inside the energy dissipation, making the optimizer to focus more in this term.

Figure 8.7 - Optimized topologies for High Reynolds Case.
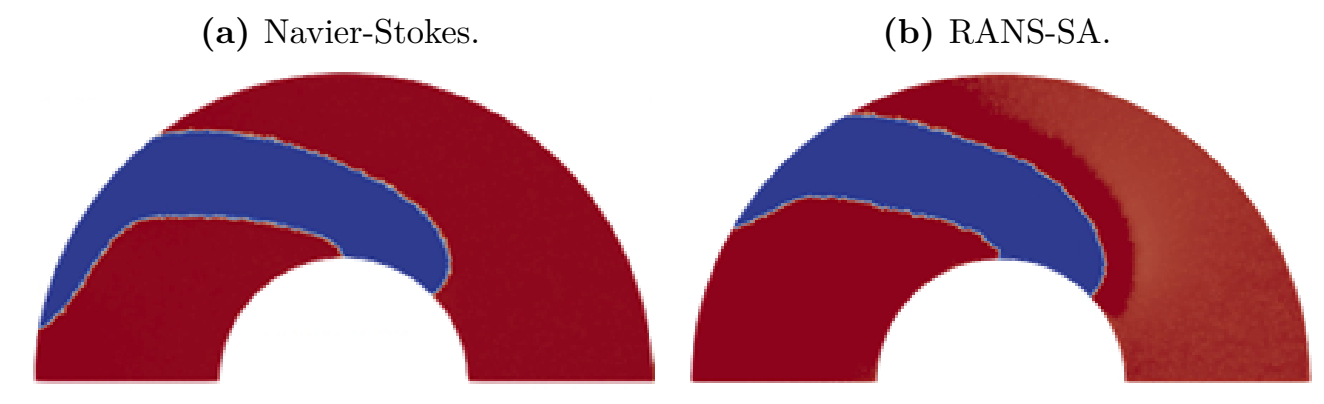

Table 8.5 - Objective function values for High Reynolds Case calculated with RANS-SA eqs..

\begin{tabular}{|c|c|c|c|c|c|}
\hline Case & $\begin{array}{c}\text { Initial guess } \\
\text { Fig. } 8.4\end{array}$ & $\begin{array}{c}\text { N-S } \\
\text { Fig. } 8.7 \mathrm{a}\end{array}$ & $\%$ & $\begin{array}{l}\text { RANS-SA } \\
\text { Fig. } 8.7 \mathrm{~b}\end{array}$ & $\%$ \\
\hline Area & 0.38 & $0 . \overline{33}$ & $-14 \%$ & 0.33 & $-14 \%$ \\
\hline Mass Flow & 29.73 & 29.73 & $0 \%$ & 29.73 & $0 \%$ \\
\hline Energy D. (Eq. 5.9$)$ & $7.05 \cdot 10^{5}$ & $6.57 \cdot 10^{5}$ & $-7 \%$ & $5.09 \cdot 10^{5}$ & $-28 \%$ \\
\hline Viscous D. (Eq. 5.10$)$ & $5.72 \cdot 10^{5}$ & $5.59 \cdot 10^{5}$ & $-2 \%$ & $4.18 \cdot 10^{5}$ & $-27 \%$ \\
\hline Friction D. (Eq. 5.11 ) & $1.33 \cdot 10^{5}$ & $0.98 \cdot 10^{5}$ & $-26 \%$ & $0.91 \cdot 10^{5}$ & $-32 \%$ \\
\hline
\end{tabular}

Table 8.5 shows the functional value for each topology evaluated with the RANS-SA equations. The $\%$ column represents the percentage change in relation to the initial guess value. Observing this table we can see that the RANS-SA model achieved a much better energy dissipation value, while the Navier-Stokes model focused mainly in the friction dissipation, which causes the flow to go by the "shorter" path, creating the topology in region with high velocity values, given that this term is proportional to $u \cdot u$. Again, this corroborates with the conclusion presented above, where the extra viscosity increases the effect of the viscous dissipation term in the optimization.

Post-processing the result by removing the solid region and smoothing the contour the topologies from Fig. 8.8 are obtained. Table 8.6 shows the functional value for the post-processed topologies. The Friction Dissipation value, in this case, is zero because the material model is not present. The contour modification caused by the post-processing seems to improve the geometry performance in this case, given that both models present better value for the energy dissipation when compared to the initial guess. This improvement may be associated with the smoother contour that removed the rugosity caused by the contour of the elements when the material model is used. However, in order to have a reliable result at the optimization stage, the RANS-SA model should be used for flows with high Reynolds numbers, given that the results with and without the material model present the same behavior. 
Figure 8.8 - Post-processed topologies for High Reynolds Case.

(a) Initial guess.

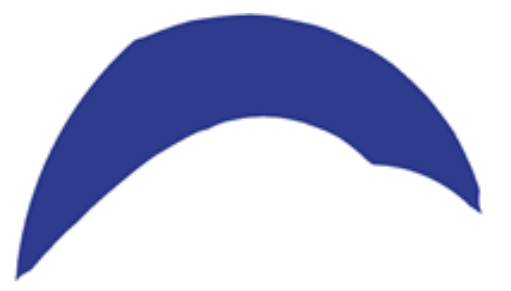

(b) Navier-Stokes.

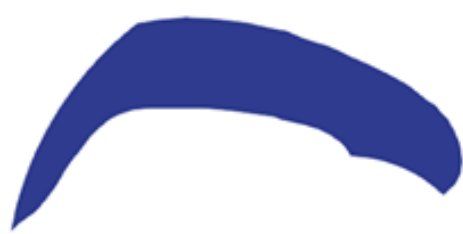

(c) RANS-SA.

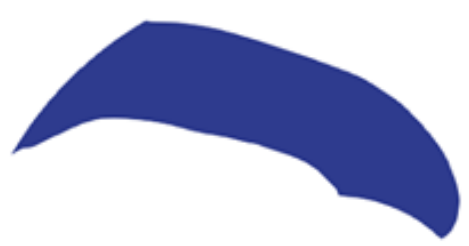

Table 8.6 - Objective function values for Post-processed High Reynolds Case calculated with RANS-SA model.

\begin{tabular}{|c|c|c|c|c|c|}
\hline Case & $\begin{array}{c}\text { Initial guess } \\
\text { Fig. } 8.8 \mathrm{a}\end{array}$ & $\begin{array}{c}\text { N-S } \\
\text { Fig. } 8.8 \mathrm{~b}\end{array}$ & $\%$ & $\begin{array}{l}\text { RANS-SA } \\
\text { Fig. } 8.8 \mathrm{c}\end{array}$ & $\%$ \\
\hline Area & 0.37 & 0.31 & $-15 \%$ & $0 . \overline{28}$ & $-23 \%$ \\
\hline Mass Flow & 29.88 & 29.04 & $-3 \%$ & 28.20 & $-6 \%$ \\
\hline Energy D. (Eq. 5.9$)$ & $4.38 \cdot 10^{5}$ & $3.32 \cdot 10^{5}$ & $-24 \%$ & $2.80 \cdot 10^{5}$ & $-36 \%$ \\
\hline Viscous D. (Eq. 5.10 & $4.38 \cdot 10^{5}$ & $3.32 \cdot 10^{5}$ & $-24 \%$ & $2.80 \cdot 10^{5}$ & $-36 \%$ \\
\hline Friction D. (Eq. $\overline{5.11}$ ) & 0 & 0 & & 0 & \\
\hline
\end{tabular}

\subsubsection{Cross Check of Results}

In order to verify the effectiveness of using the turbulence model in the optimization process it is necessary to compare the resulting topologies, obtained with Navier-Stokes and RANS-SA models, and evaluate them with both models so the conclusion derived in the previous sections are not biased. Hence cross check consists of performing four steps for each Reynolds number:

(I) Evaluate the Navier-Stokes topologies (Figs. 8.6b and 8.8b;

(a) With Navier-Stokes Equations;

(b) With RANS-SA Equations;

(II) Evaluate the RANS-SA topologies (Figs. 8.6c and 8.8c):

(a) With Navier-Stokes Equations;

(b) With RANS-SA Equations;

The results of the cross-check are shown in Tab. 8.7. It is noticeable that the topologies obtained with the RANS-SA perform better in all cases. The viscous dissipation values for low Reynolds number are very close which is aligned with the previous conclusion that for low Reynolds any of the models could be used, even though they do not result in the 
same topology. For the high Reynolds number, the difference is more relevant, given that the magnitude order is $10^{5}$, and then the RANS-SA model should be used.

Table 8.7 - Cross-check: Objective function values for Post-processed topologies.

\begin{tabular}{llrcc}
\hline \multirow{2}{*}{ Reynolds Number } & \multirow{2}{*}{ Model at Design } & \multirow{2}{*}{ Figure } & \multicolumn{2}{c}{ Model at Evaluation } \\
& & & N-S & RANS-SA \\
\hline Low & N-S & $8.6 \mathrm{~b}$ & 10.76 & 22.12 \\
& RANS-SA & $8.6 \mathrm{c}$ & 9.84 & 20.82 \\
\multirow{2}{*}{ High } & & & & \\
& N-S & $8.8 \mathrm{~b}$ & $2.21 \cdot 10^{5}$ & $3.32 \cdot 10^{5}$ \\
& RANS-SA & $8.8 \mathrm{c}$ & $1.80 \cdot 10^{5}$ & $2.80 \cdot 10^{5}$ \\
\hline
\end{tabular}

\subsection{Impeller Design (Non-Newtonian Model)}

This section presents the model and results for the rotor design considering the Non-newtonian fluid. The domain is extended to have a region filled with fluid at the external radius $\left(\Omega_{f i x}\right)$ where no optimization is performed. This is done in order to try to represent a situation in which the rotor sends fluid to the volute.

The domain dimensions are inner radius of $7[\mathrm{~mm}]$, middle radius of $15[\mathrm{~mm}]\left(\Omega_{\text {opt }}\right)$, and outer radius of $18[\mathrm{~mm}]$. Two circular sections are analysed: $\beta_{\text {geo }}=120^{\circ}$ and $\beta_{\text {geo }}=180^{\circ}$. The boundary conditions are shown in Fig.8.9. The domain outlet has zero tension condition, the walls have a no-slip condition, and the inlet has an aperture of $\beta_{\text {in }}=50^{\circ}$ with a parabolic velocity profile angled $\left(\theta_{u_{i n}}\right)$ in relation to the boundary normal direction. A rotation of $3000[\mathrm{rpm}]$ and a inlet velocity $\left(u_{i n}\right)$ calculated to attend the mass flow of $3.0[l / \mathrm{min}]$ are used. The inlet velocity is angled so the relative velocity can represent a normal inlet velocity at the absolute reference frame. The ideal angle at this scale and rotation would be around $85^{\circ}$, however, this brings some computational issues when calculating the velocity magnitude due to $\cos \theta_{u_{i n}}$ being close to zero $\left(u_{i n}=\frac{u_{n}}{\cos \theta_{u_{i n}}} ; u_{n}\right.$ is the normal velocity calculated with the mass flow). Hence, the angle used is $60^{\circ}$.

Still, due to computational issues, the mass flow value is lower than the values used in a real scale adult VAD (BARRETT et al., 2010). However, the optimized topologies are not expected to change for slightly higher values $(5.0[\mathrm{l} / \mathrm{min}])$. 
Figure 8.9 - Boundary conditions for the rotor optimization.

$$
\begin{aligned}
& r_{\text {in }}=7 \mathrm{~mm} \\
& r_{\text {opt }}=15 \mathrm{~mm} \\
& r_{\text {out }}=18 \mathrm{~mm} \\
& \theta_{u_{\text {in }}}=60^{\circ} \\
& \omega=3000 \mathrm{rpm} \\
& u_{\text {in }}=3 \mathrm{l} / \mathrm{min}
\end{aligned}
$$

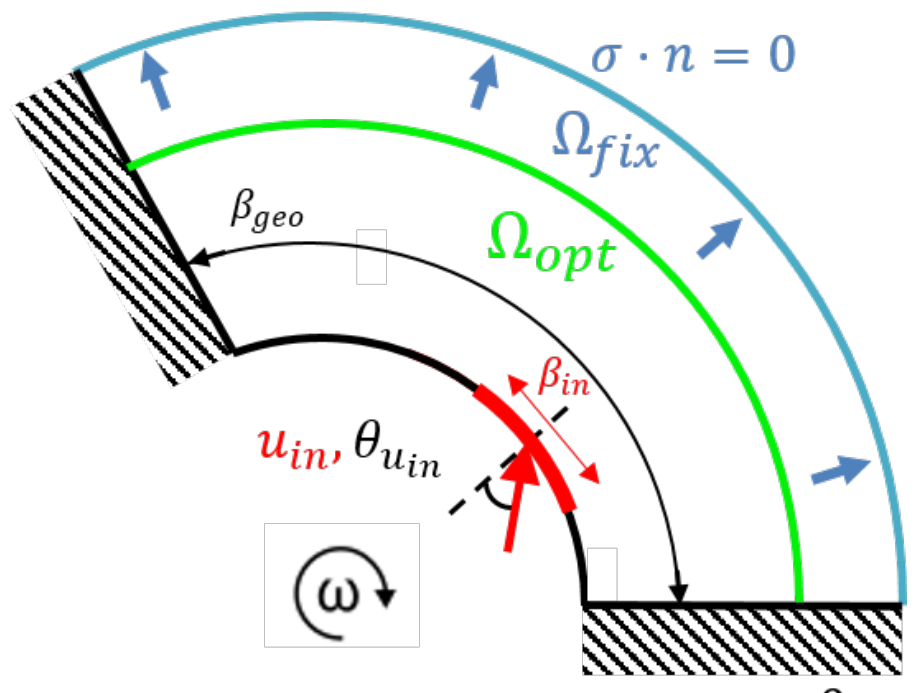

$$
u_{\text {wall }}=0
$$

Table 8.8 - Inputs for non-Newtonian Impeller Optimization.

\begin{tabular}{lcc}
\hline Parameter & Value & Unit \\
\hline$r_{\text {in }}$ & 0.7 & {$[\mathrm{~cm}]$} \\
$r_{\text {opt }}$ & 1.5 & {$[\mathrm{~cm}]$} \\
$r_{\text {out }}$ & 1.8 & {$[\mathrm{~cm}]$} \\
$\beta_{\text {in }}$ & 50 & {$\left[{ }^{\circ}\right]$} \\
$\theta_{u_{\text {in }}}$ & 60 & {$\left[{ }^{\circ}\right]$} \\
$\rho$ & 1.06 & {$\left[\mathrm{~g} / \mathrm{cm}^{3}\right]$} \\
& & \\
$\mu(\dot{\gamma})$ & Carreau $-\mathrm{Yasuda}$ \\
$\mu_{\infty}$ & 0.16 & {$[\mathrm{~Pa} \cdot \mathrm{s}]$} \\
$\mu_{0}$ & 0.0035 & {$[\mathrm{~Pa} \cdot \mathrm{s}]$} \\
$\lambda$ & 8.2 & \\
$a$ & 0.64 & \\
$n$ & 0.2128 & \\
& \multicolumn{3}{|c}{} \\
$\left|u_{\text {in }}\right|$ & 39.79 & {$[\mathrm{~cm} / \mathrm{s}]$} \\
$\left|u_{\text {wall }}\right|$ & 0.0 & {$[\mathrm{~cm} / \mathrm{s}]$} \\
$\omega$ & 314.2 & {$[\mathrm{rad} / \mathrm{s}]$} \\
\hline
\end{tabular}




\subsubsection{Design Domain of 120 degrees}

This section shows the optimization results for the domain consisting of a circular section with $\beta_{\text {geo }}=120^{\circ}$. Three cases are analyzed by changing the optimization constants in accord to Tab. 8.9. The optimization is performed to minimize energy dissipation as an indirect way to minimize hemolysis. The optimized topologies are shown in Fig. 8.10 as well as an arbitrary straight blade for comparison. In this figure, the blue region $(\alpha=1)$ represents the fluid domain and the red region, the solid domain $(\alpha=0)$.

Table 8.9 - Optimization inputs for $\beta_{\text {in }}=120^{\circ}$.

\begin{tabular}{lccc}
\hline Parameter & Case 1 & Case 2 & Case 3 \\
\hline Volume Constraint & $30 \%$ & $30 \%$ & $30 \%$ \\
Initial Distribution & 0.3 & 0.3 & 0.3 \\
$k_{\max }$ & 1,000 & 1,000 & 10,000 \\
$q$ & $0.01-0.1$ & 0.1 & $0.01-0.1$ \\
\hline
\end{tabular}

Figures 8.10b, 8.10c, and 8.10d we can see that the algorithm tends to straighten the topology as $k_{\max }$ and $q$ increase. This indicates that with the increase of the Friction dissipation (Eq. 5.11) the topology tends to choose the shortest flow path from the inlet to the fixed domain.

Figure 8.10 - Straight blade and Optimized topologies for $\beta_{\text {geo }}=120^{\circ}$.

(a) Straight Blade.

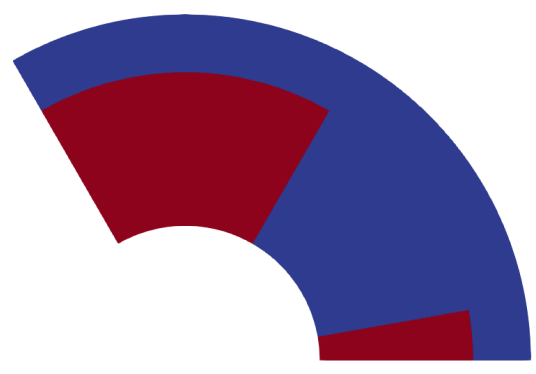

(c) Case 2 .

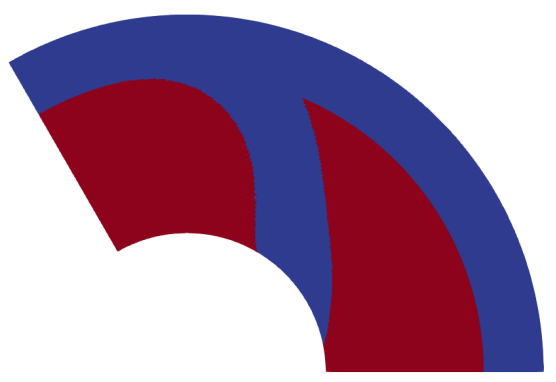

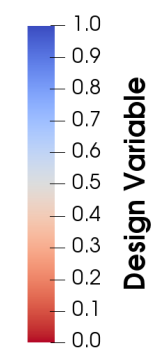

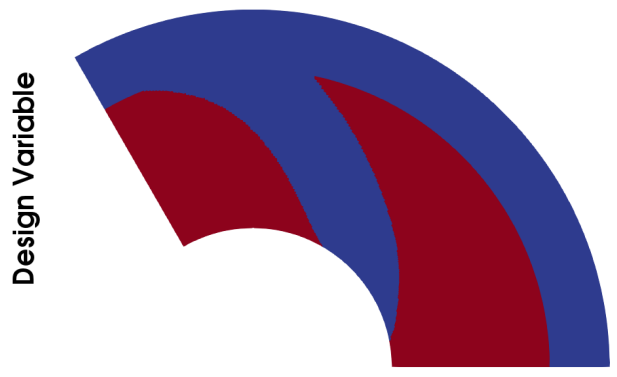

(d) Case 3 .

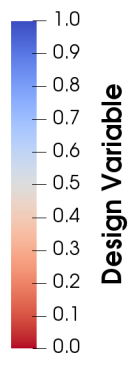

(b) Case 1.

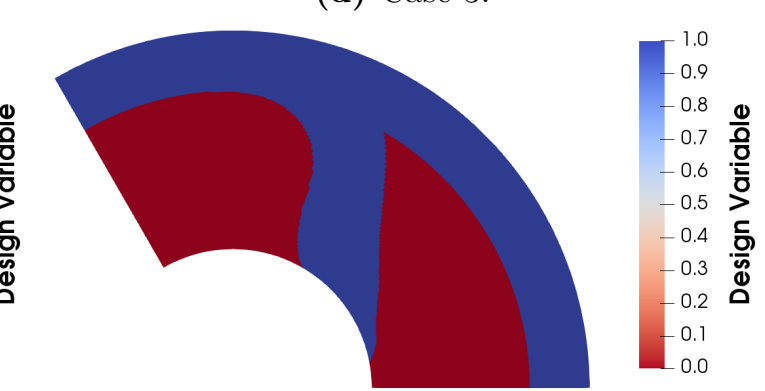

The optimization convergence curve is shown in Fig. 8.11. The curves for case 1 and 3 are smoother than for the second case. Due to the change in the value of $q$ at iteration 100 a spike at the function value occurs. 
Figure 8.11 - Convergence curves for $\beta_{\text {geo }}=120^{\circ}$.

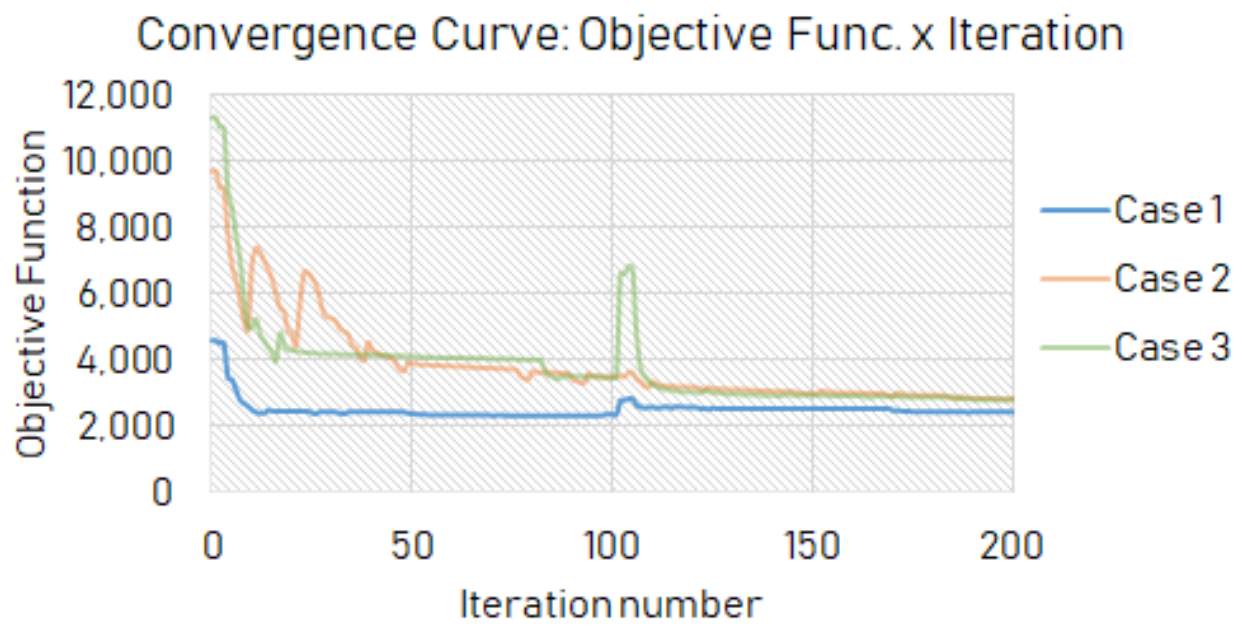

The velocity plot of the Straight Blade (Fig. 8.10a) shows a large region with flow re-circulation at the right, while most of the fluid flows near the left side. The re-circulation is not present in the optimized topologies. The passage from the optimization domain to the fixed domain is much more smooth in the optimized blades and it is a direct effect of the chosen objective function, which lowers the velocity strain rate. The pressure fields (Fig. 8.13) do not show much change among the cases.

Figure 8.12 - Velocity field for Straight blade and Optimized topologies $\left(\beta_{\text {geo }}=120^{\circ}\right)$.

(a) Straight Blade.

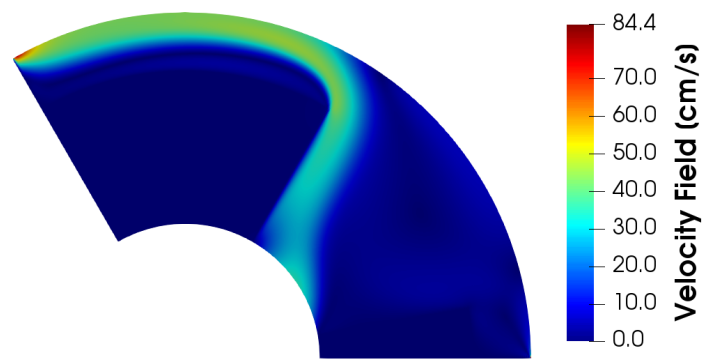

(c) Case 2 .

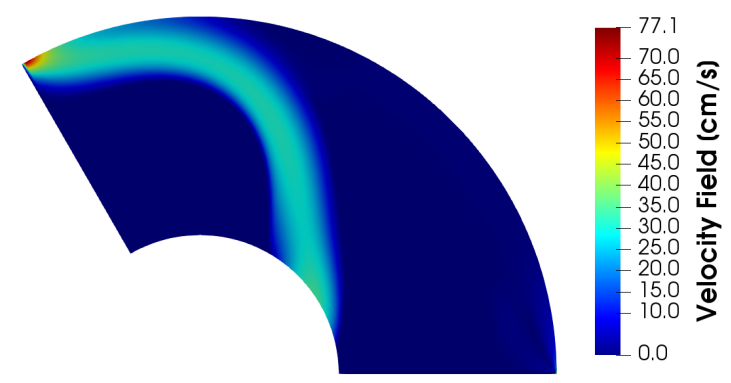

(b) Case 1 .

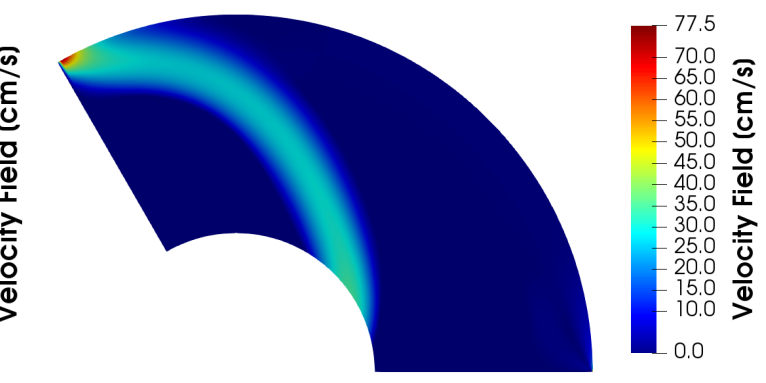

(d) Case 3 .

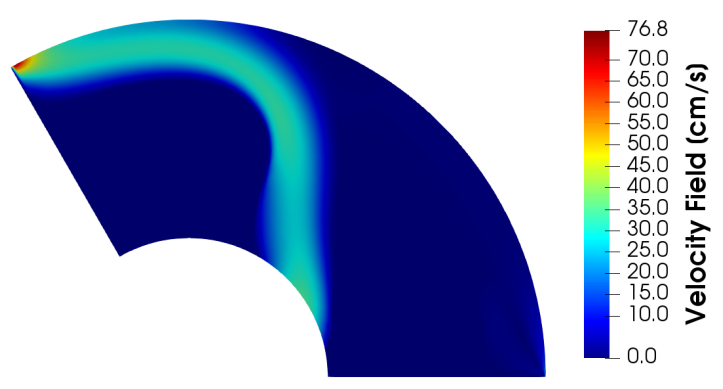


Figure 8.13 - Pressure field for Straight blade and Optimized topologies $\left(\beta_{\text {geo }}=120^{\circ}\right)$.

(a) Straight Blade.

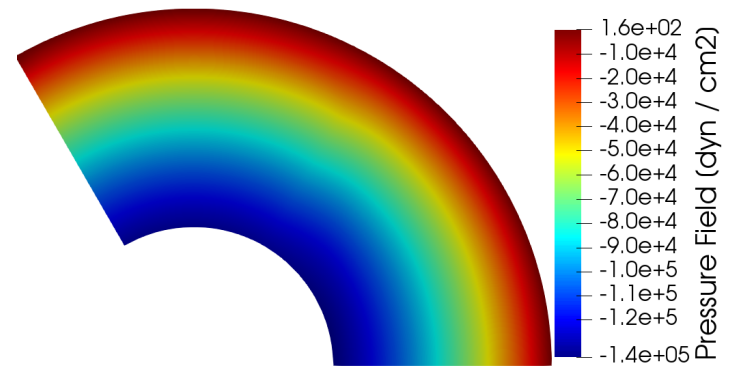

(c) Case 2 .

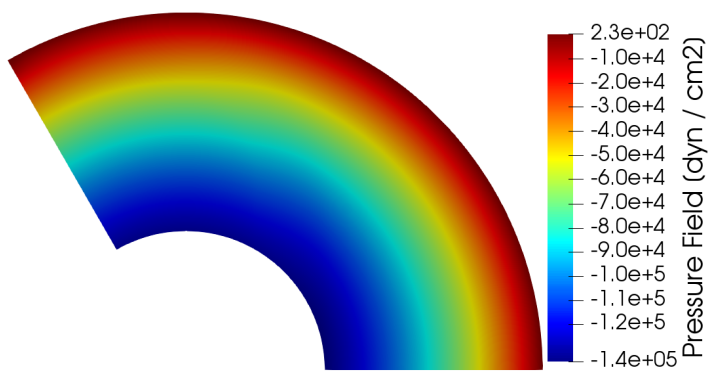

(b) Case 1 .

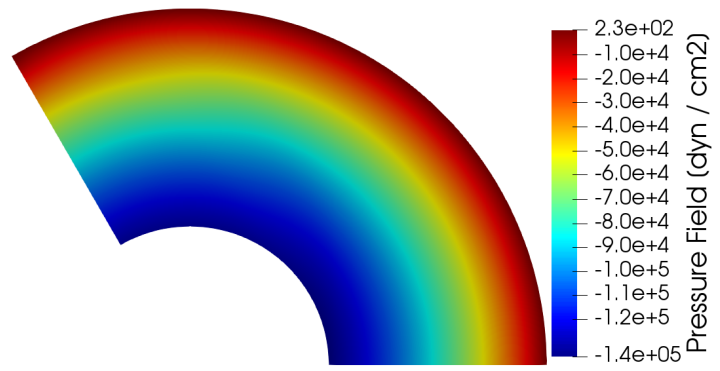

(d) Case 3 .

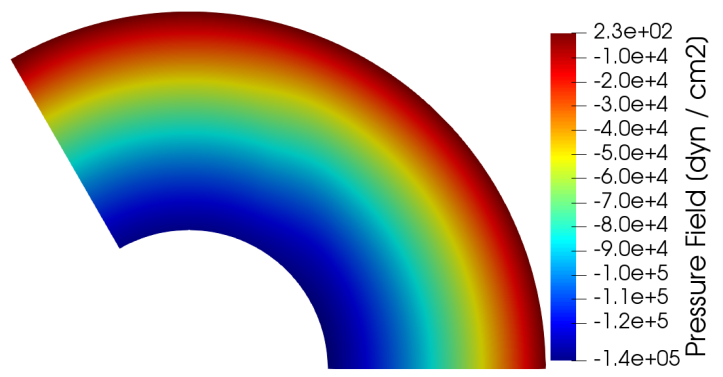

Figure 8.14 - Viscosity field for Straight blade and Optimized topologies $\left(\beta_{\text {geo }}=120^{\circ}\right)$.

(a) Straight Blade.

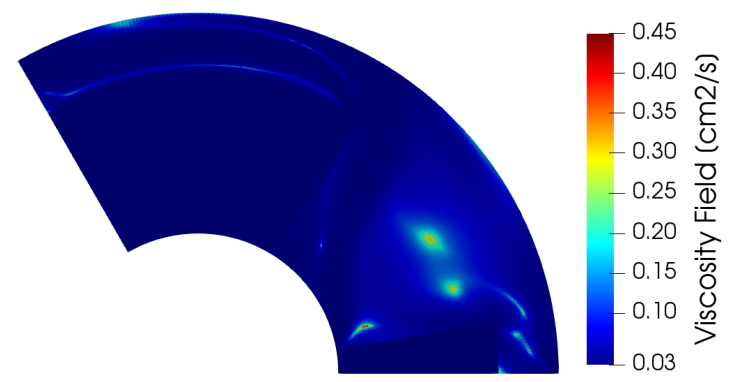

(c) Case 2 .

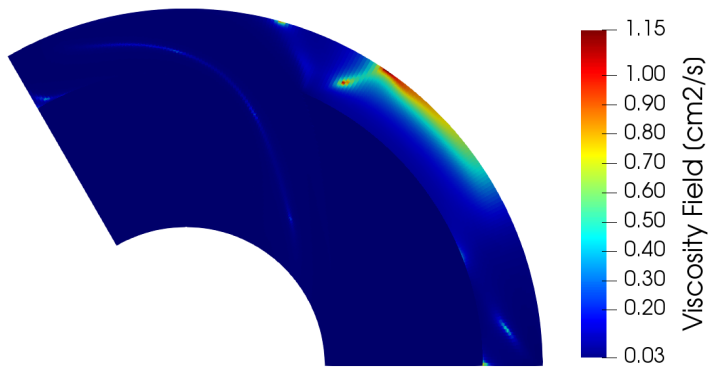

(b) Case 1 .

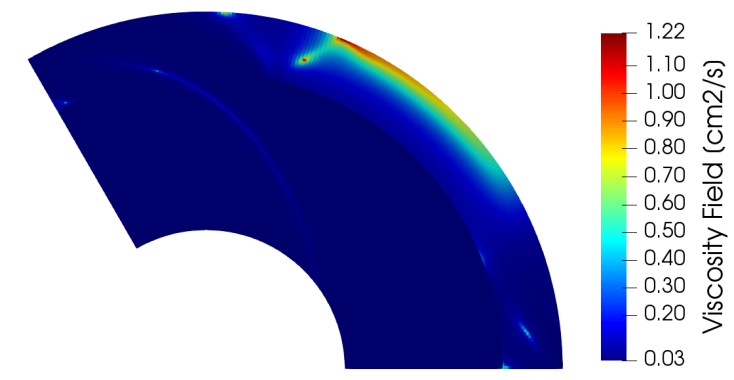

(d) Case 3 .

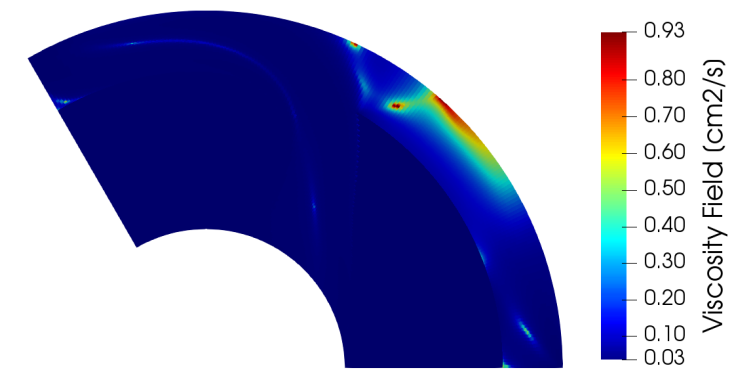

The viscosity plots (Fig. 8.14) are coherent with the velocity plots, in which the highest viscosity occurs at the center of the channel, where the gradients are lower. This effect is expected due to the use of the Carrau-Yasuda model. The re-circulation that arises in the Straight Blade causes an increase of viscosity at the center of the vortex. The viscosity field for the optimized blades shows a high viscosity at a region with almost no velocity 
and consequently with low gradients.

Figure 8.15 - Energy dissipation (point-wise) for Straight blade and Optimized topologies $\left(\beta_{\text {geo }}=120^{\circ}\right)$.

(a) Straight Blade.

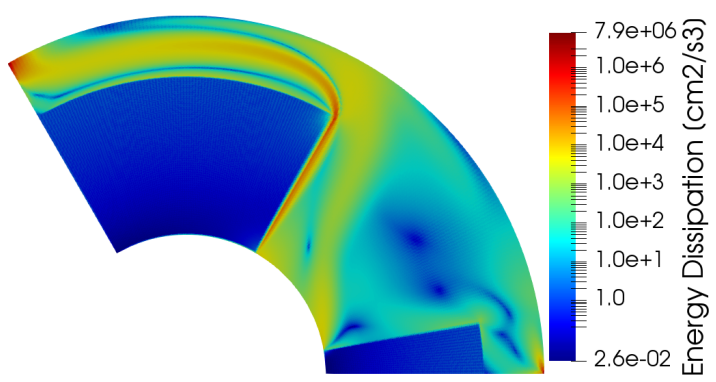

(c) Case 2 .

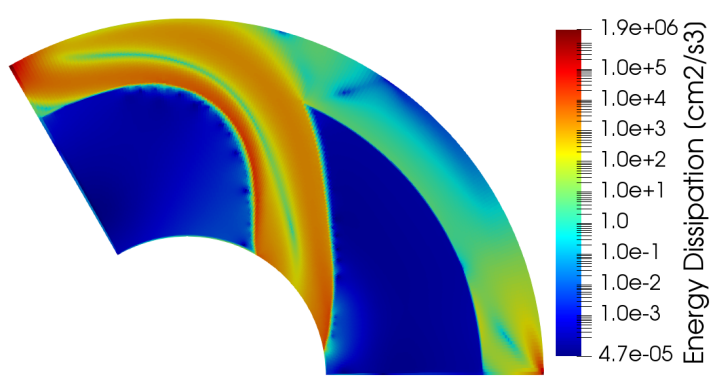

(b) Case 1 .

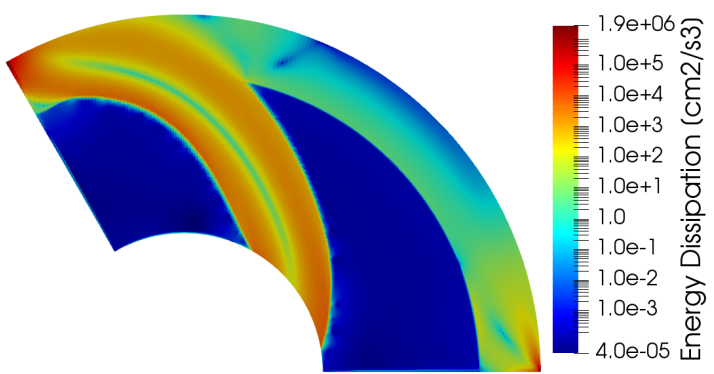

(d) Case 3 .

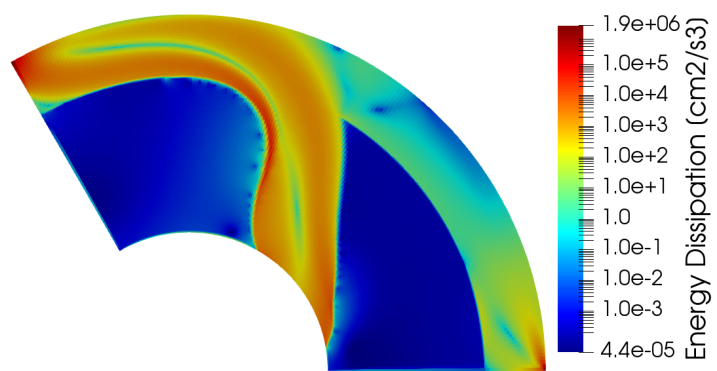

The energy dissipation and vorticity functionals can be analyzed with a point-wise plot, as shown in Figs. 8.15 and 8.16, respectively. Both functionals are proportional to the velocity gradient, and this is justified by looking at the shape of the plots, which are very similar for each case. One can notice that the lowest values occur at the center of the blades, where, again, the velocity gradients are lower. There is a small re-circulation near the right lower corner of the domain that is identified by the high velocity gradients. These regions are an indirect effect of using the rotational body forces in the entire domain and having an area surrounded by an outlet and no-slip (both by the wall and by the solid material) boundary conditions.

Observing the energy dissipation plot of the Straight blade (Fig. 8.15a), we can see that the peak value is much higher than the ones present in the optimized blades. Also, the optimized blade of Case 1 (Fig. 8.15b) has lower values at the left wall, given that it has a smoother curvature. 
Figure 8.16 - Vorticity (point-wise) for Straight blade and Optimized topologies $\left(\beta_{\text {geo }}=120^{\circ}\right)$.

(a) Straight Blade.

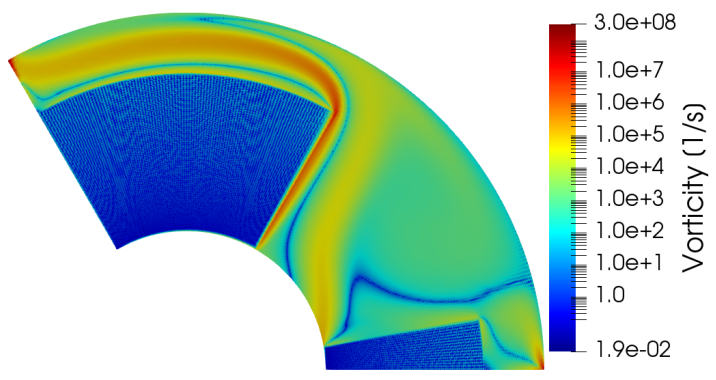

(c) Case 2 .

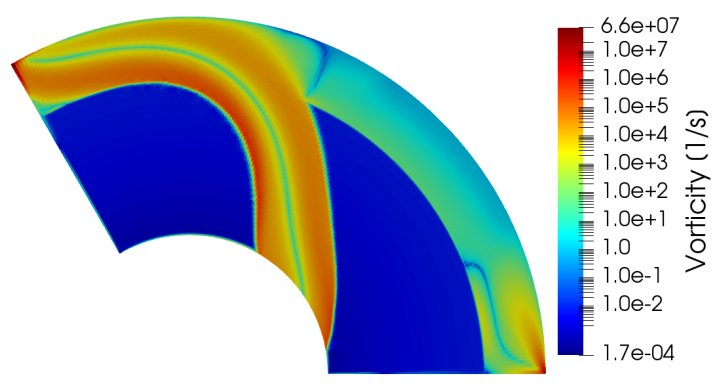

(b) Case 1.

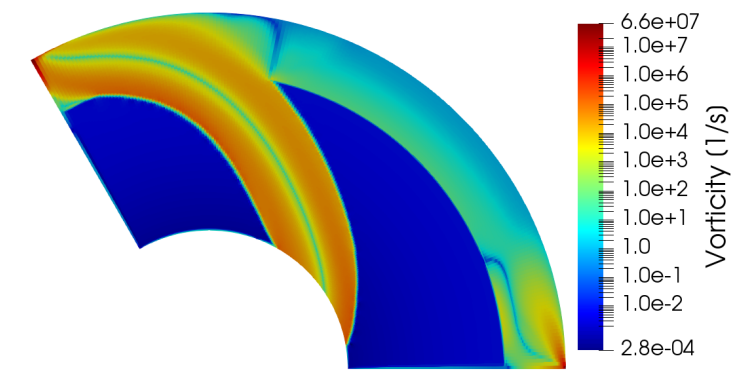

(d) Case 3 .

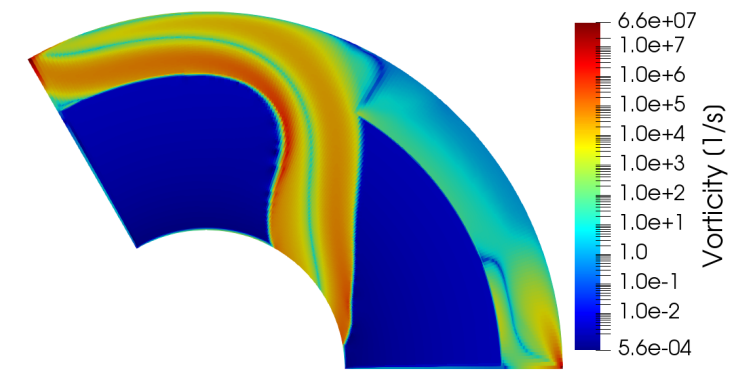

Table 8.10 shows the functional values for the three cases and a comparison with the straight blade. The \% column shows the percent change in relation to the straight blade. As we can see, the pressure field presents almost no change among the cases. The optimized blades have at least 30\% less energy dissipation than the straight blade. The friction dissipation is the most affected in the optimization, given that it presents value reductions as high as $95 \%$, however, this has small meaning for the impeller to be built, given that it can be interpreted as a numerical artifice to remove gray regions. The vorticity values are also improved by at least 34\%. The optimized blade from Case 1 seems to have the best performance for the given functionals.

Table 8.10 - Functional values of Straight and Optimized blades with $\beta_{\text {in }}=120^{\circ}$.

\begin{tabular}{lccccccc}
\hline & Straight Blade & Case 1 & $\%$ & Case 2 & $\%$ & Case 3 & $\%$ \\
\hline Area & 1.81 & 1.56 & -14 & 1.45 & -20 & 1.44 & -20 \\
Mass Flow & 2.99 & 2.99 & 0 & 2.99 & 0 & 2.99 & 0 \\
Inlet Pressure $\left(\cdot 10^{5}\right)$ & -1.33 & -1.34 & 0 & -1.33 & 0 & -1.33 & 0 \\
Energy Dissip. $\left(\cdot 10^{3}\right)$ & 4.09 & 2.38 & -42 & 2.69 & -34 & 2.72 & -33 \\
Viscous Dissip. $\left(\cdot 10^{3}\right)$ & 3.81 & 2.37 & -38 & 2.65 & -31 & 2.67 & -30 \\
Friction Dissip. $\left(\cdot 10^{2}\right)$ & 2.71 & 0.12 & -95 & 0.41 & -85 & 0.52 & -81 \\
Vorticity $\left(\cdot 10^{5}\right)$ & 0.95 & 0.54 & -42 & 0.62 & -35 & 0.62 & -34 \\
\hline
\end{tabular}

As indicated in the previous chapter, a more insightful analysis can be performed when the topologies are isolated, the contour is smoothed, and the effect of the material 
model is removed. Thus, the isolated blades are shown in Fig. 8.17. Table 8.11 shows the functional values for the isolated blades. The $\%$ column shows the value in relation to the straight blade. The optimized blades from Cases 1 (Fig. 8.17b) and 3 (Fig. 8.17d) have energy dissipation values almost $30 \%$ lower than the straight blade, while the one from Case 2 (Fig. 8.17c has an improvement of $14 \%$. The vorticity functional is also improved indirectly, and Case 1 shows a value $41 \%$ lower than the traditional straight blade. It is important to notice that the friction dissipation is zero here, given that the isolated blades do not have the material model. Hence, the energy dissipation is given only by the viscous dissipation.

Table 8.11 - Functional values of Isolated Straight and Optimized blades with $\beta_{i n}=120^{\circ}$.

\begin{tabular}{lccccccc}
\hline & Straight Blade & Case 1 & $\%$ & Case 2 & $\%$ & Case 3 & $\%$ \\
\hline Area & 1.78 & 1.54 & -13 & 1.44 & -19 & 1.42 & -20 \\
Mass Flow & 2.98 & 2.97 & 0.3 & 2.98 & 0.1 & 2.99 & 0.2 \\
Inlet Pressure $\left(\cdot 10^{5}\right)$ & -1.33 & -1.33 & 0 & -1.33 & 0 & -1.33 & 0 \\
Energy Dissip. $\left(\cdot 10^{3}\right)$ & 3.49 & 2.27 & -35 & 2.98 & -14 & 2.48 & -29 \\
Viscous Dissip. $\left(\cdot 10^{3}\right)$ & 3.49 & 2.27 & -35 & 2.98 & -14 & 2.48 & -29 \\
Friction Dissip. & 0.00 & 0.00 & 0 & 0.00 & 0 & 0.00 & 0 \\
Vorticity $\left(\cdot 10^{5}\right)$ & 0.83 & 0.49 & -41 & 0.67 & -19 & 0.55 & -34 \\
\hline
\end{tabular}

Finally, the complete rotor can be formed by replicating the blades with angular periodicity, and the impellers are shown in Fig. 8.18. Notice that the blue regions in this figure represent the fluid, hence, the blades are defined by the white portion.

Figure 8.17 - Isolated Straight and Optimized blades for $\beta_{\text {geo }}=120^{\circ}$.

(a) Straight Blade.

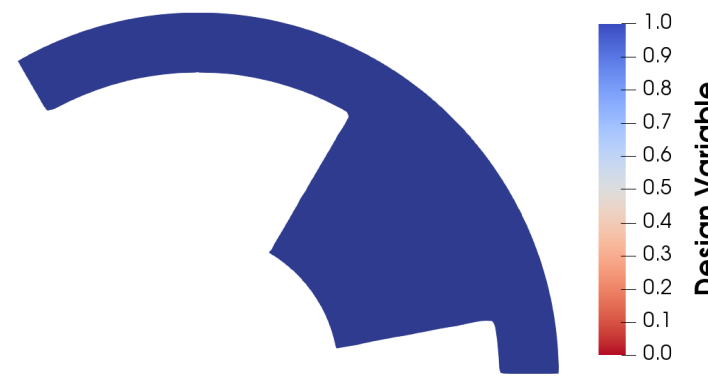

(c) Case 2 .

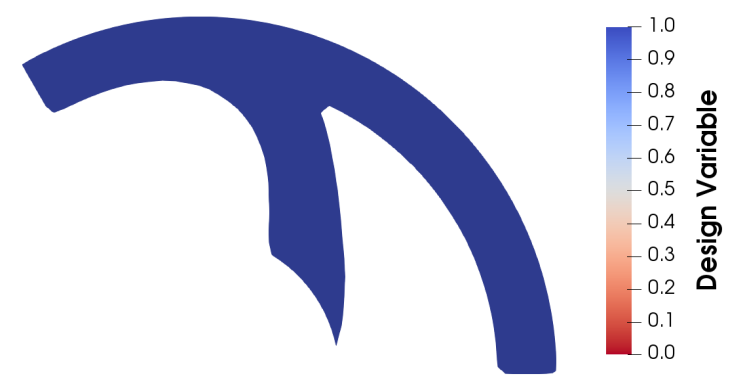

(b) Case 1.

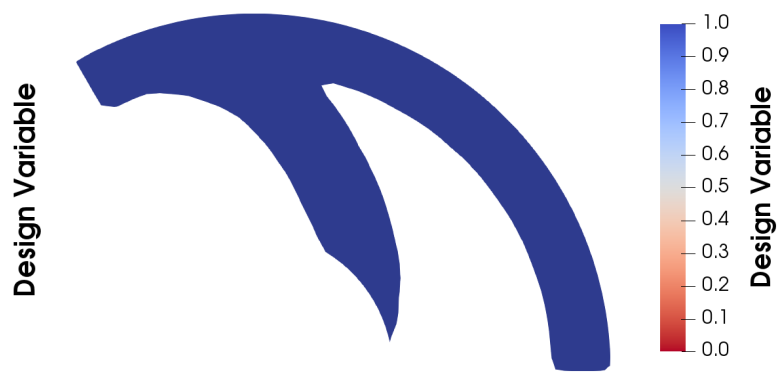

(d) Case 3 .

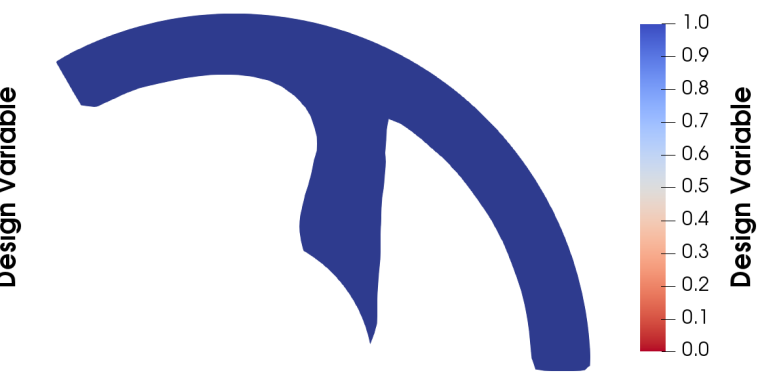


Figure 8.18 - Complete impellers for Straight and Optimized blades $\left(\beta_{\text {geo }}=120^{\circ}\right)$.

(a) Straight Blade.

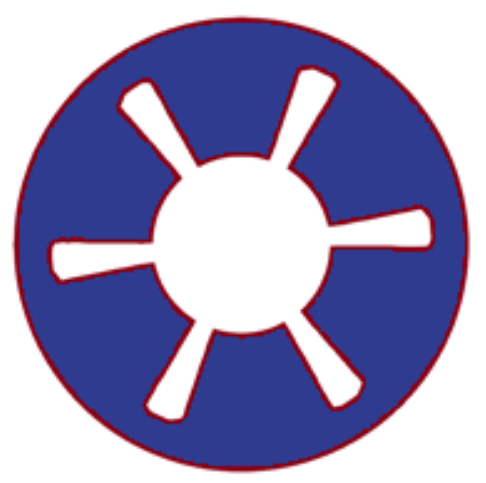

(c) Case 2 .

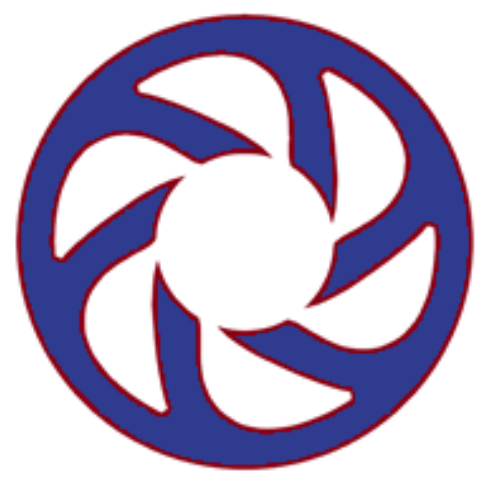

(b) Case 1.

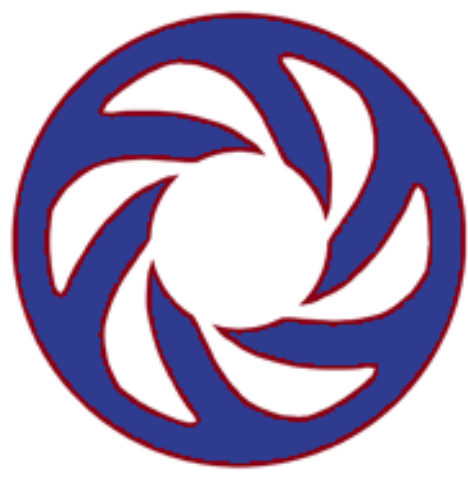

(d) Case 3 .

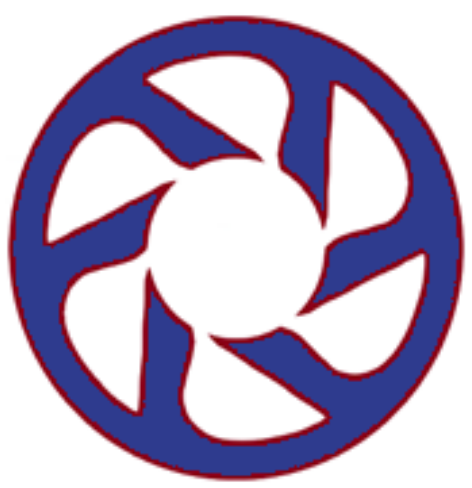

\subsubsection{Design Domain of 180 degrees}

This section shows the optimization results for the domain consisting of a circular section with $\beta_{\text {geo }}=180^{\circ}$. Only cases 1 and 3 are analyzed, and the optimization is performed aiming to minimize the energy dissipation. The optimized topologies are shown in Fig. 8.19 as well as an arbitrary straight blade for comparison. The optimized blades are very similar to the ones presented in Sec. 8.2.1 indicating that the algorithm is not being affected by the geometry angle $\beta_{\text {geo }}$. In order to further improve the comparison a new straight blade (SB60) is analyzed, this blade is obtained by rotating the blades by $60^{\circ}$ so the inlet velocity would be aligned with the blade $\left(\beta_{u_{i n}}\right)$.

In Figs. $8.19 \mathrm{~b}$ and $8.19 \mathrm{~d}$ we can see the same tendency to straighten the topology as $k_{\text {max }}$ and $q$ increases. Thus, the case of the $60^{\circ}$ straight blade (Fig. 8.19c) is an attempt to verify this. Table 8.12 reveals that indeed this inclination of the blade reduces the energy dissipation functional value.

The optimization convergence curve is shown in Fig. 8.20. The curves for case 1 and 3 are very similar to the curves from the previous section. Again, the change in the value of $q$ at iteration 100 causes a spike at the function value. 
Figure 8.19 - Straight blade and Optimized topologies for $\beta_{\text {geo }}=180^{\circ}$.

(a) Straight Blade.

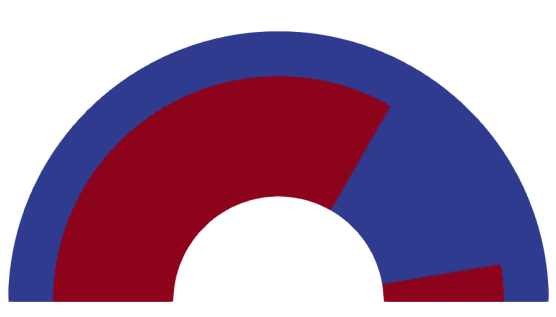

(c) Straight Blade $60^{\circ}$.

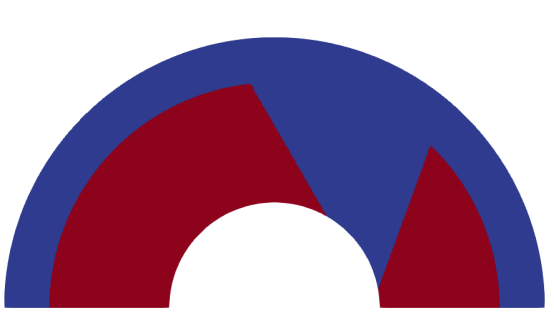

(b) Case 1 .
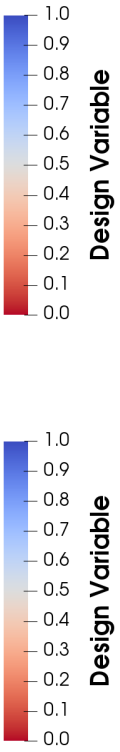

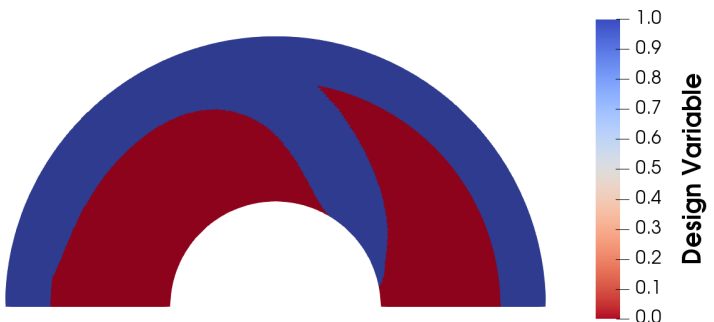

(d) Case 3 .

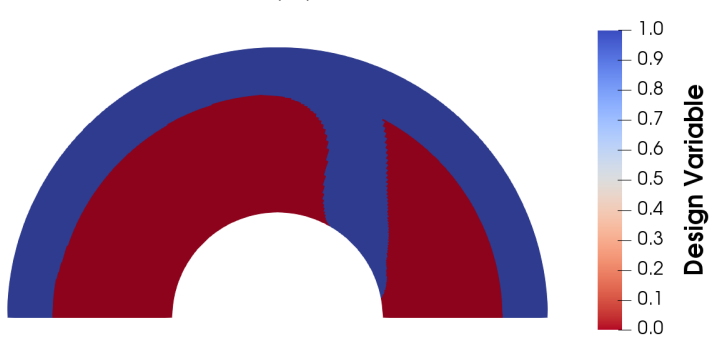

Figure 8.20 - Convergence curves for $\beta_{\text {geo }}=180^{\circ}$.

\section{Convergence Curve: Objective Func. $x$ Iteration}

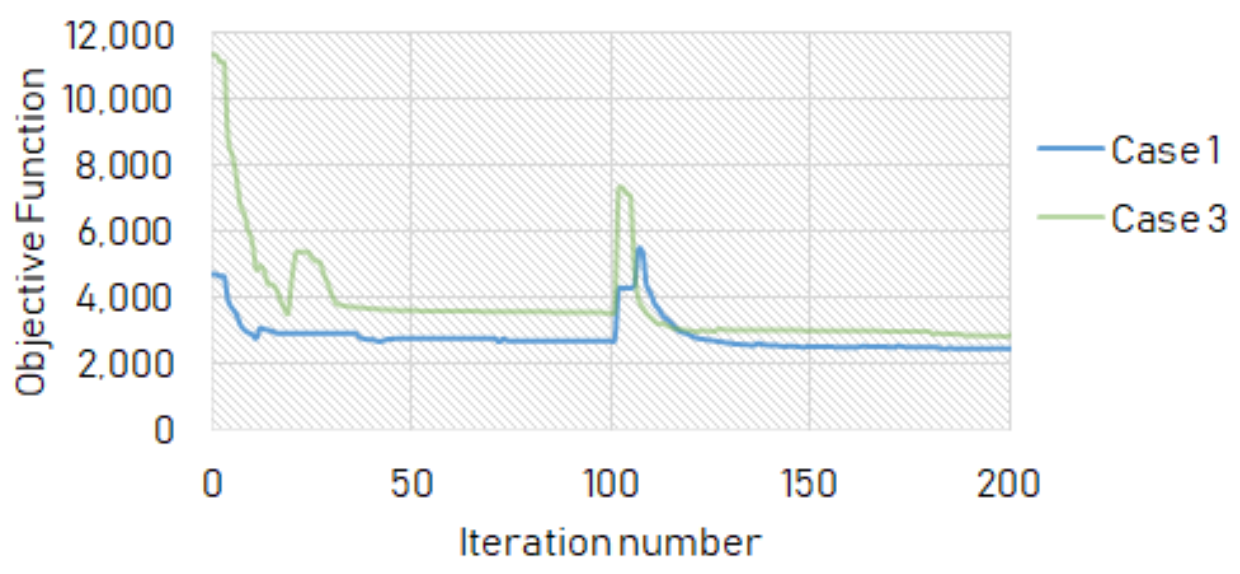

Table 8.12 - Functional values of Straight and Optimized blades with $\beta_{\text {in }}=180^{\circ}$.

\begin{tabular}{lccccccc}
\hline & Straight B. & SB60 & $\%$ & Case 1 & $\%$ & Case 3 & $\%$ \\
\hline Area & 2.33 & 2.33 & 0 & 2.24 & -4 & 2.03 & -13 \\
Mass Flow & 2.99 & 2.99 & 0 & 2.99 & 0 & 2.99 & 0 \\
Inlet Pressure $\left(\cdot 10^{5}\right)$ & -1.33 & -1.33 & 0 & -1.34 & 0 & -1.33 & 0 \\
Energy Dissip. $\left(\cdot 10^{3}\right)$ & 4.53 & 3.10 & -32 & 2.43 & -46 & 2.76 & -39 \\
Viscous Dissip. $\left(\cdot 10^{3}\right)$ & 4.19 & 3.02 & -28 & 2.42 & -42 & 2.71 & -35 \\
Friction Dissip. $\left(\cdot 10^{2}\right)$ & 3.45 & 0.89 & -74 & 0.13 & -96 & 0.47 & -86 \\
Vorticity $\left(\cdot 10^{5}\right)$ & 1.04 & 0.69 & -34 & 0.55 & -48 & 0.63 & -40 \\
\hline
\end{tabular}


The velocity plots (Fig. 8.21) shows the same behavior as presented in the previous section. The case SB60 still presents a large region with flow re-circulation at the right; however, the velocity profile is smoother than the pure radial blade. The peak velocity is also higher in this case. The pressure fields (Fig. 8.22) do not show much change among the cases, except for the SB60 blade, in which the peak value is higher.

Figure 8.21 - Velocity field for Straight blade and Optimized topologies $\left(\beta_{g e o}=180^{\circ}\right)$.

(a) Straight Blade.

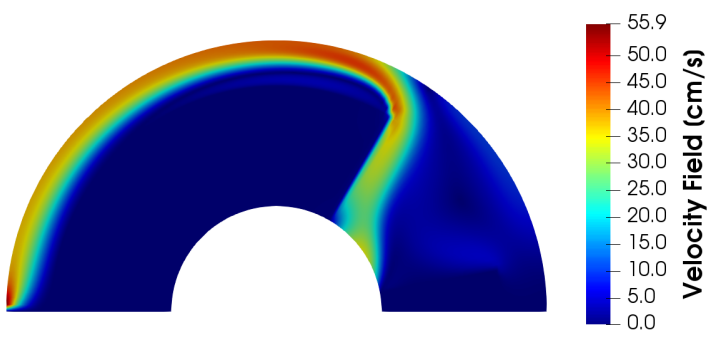

(c) Straight Blade $60^{\circ}$.
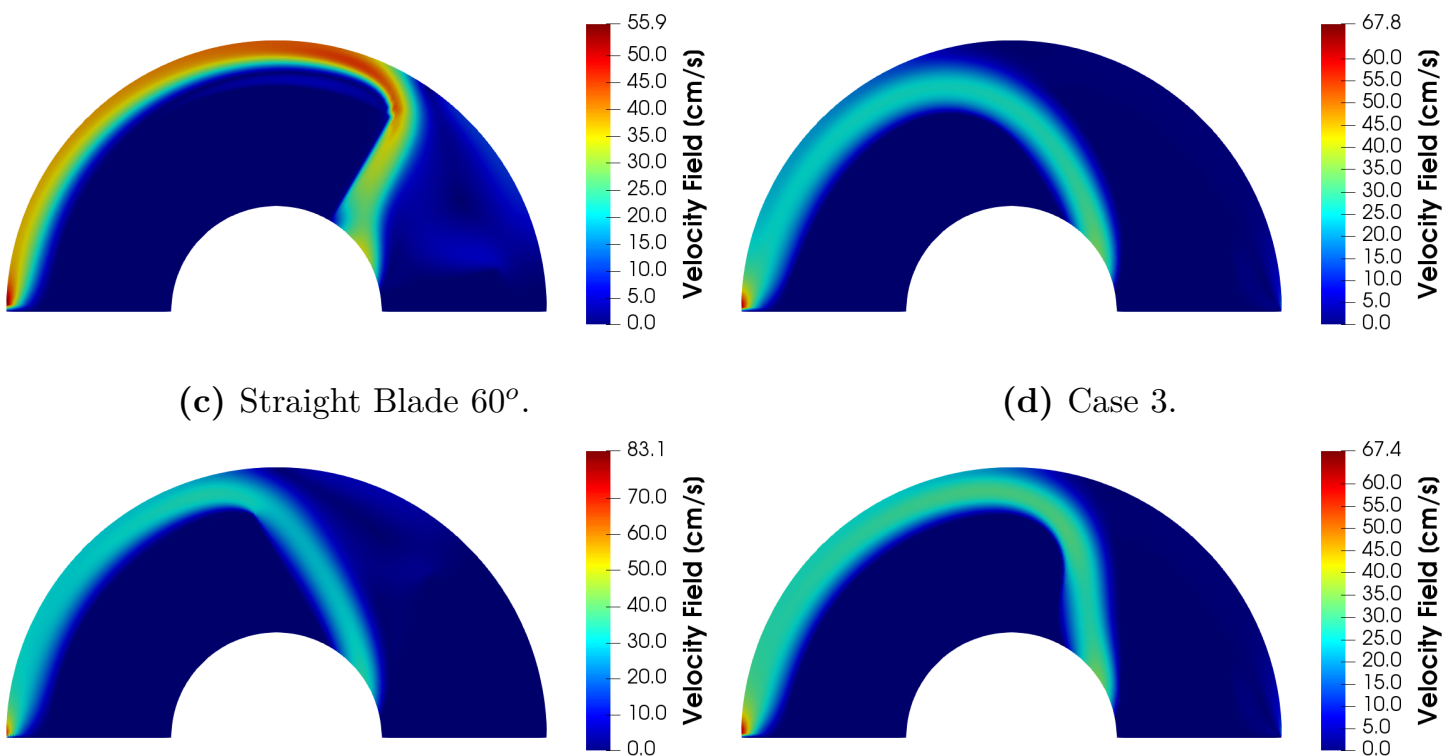

(d) Case 3 .

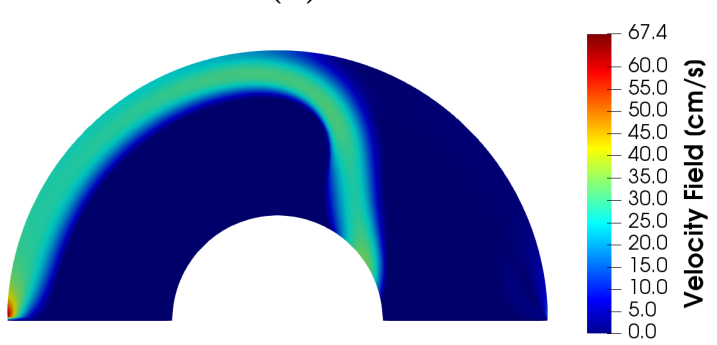

Figure 8.22 - Pressure field for Straight blade and Optimized topologies $\left(\beta_{\text {geo }}=180^{\circ}\right)$.

(a) Straight Blade.

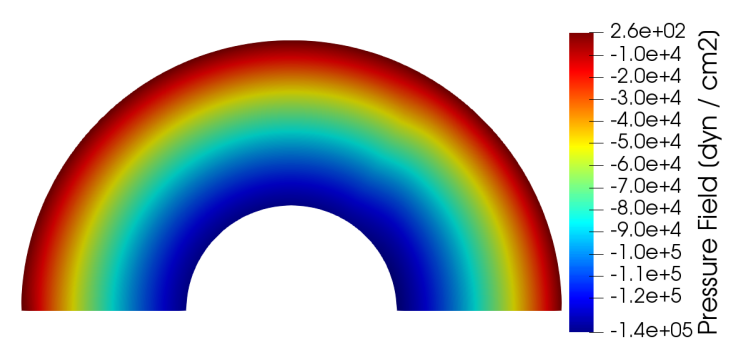

(c) Straight Blade $60^{\circ}$.

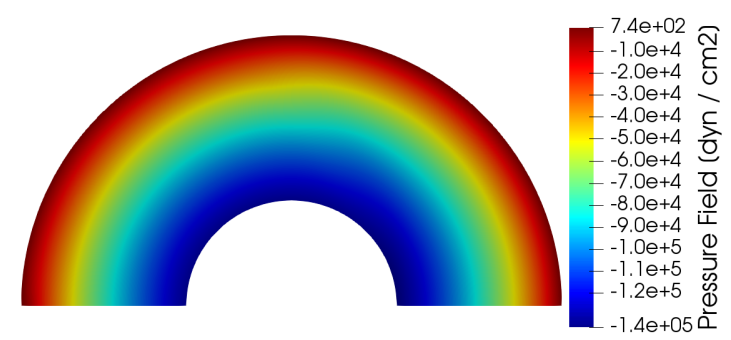

(b) Case 1 .

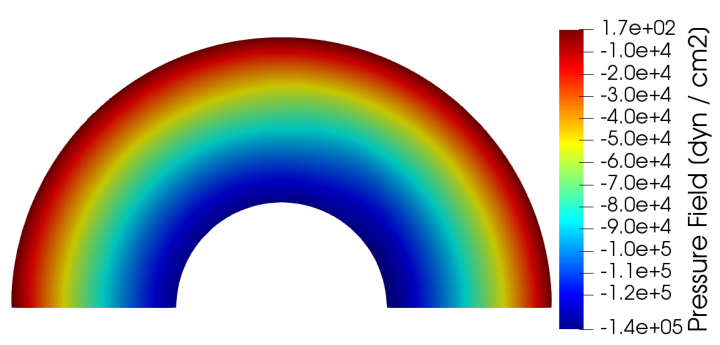

(d) Case 3 .

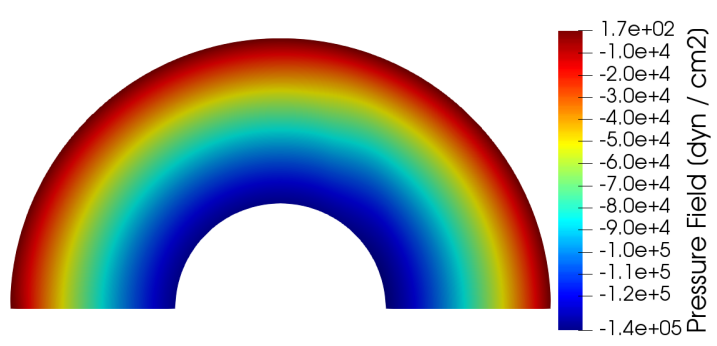

The viscosity plots (Fig. 8.23) are coherent with the viscosity plots of the previous section (Fig. 8.14). The SB60 case shows larger areas with high viscosity resulting from 
low velocity gradients.

Figure 8.23 - Viscosity field for Straight blade and Optimized topologies $\left(\beta_{\text {geo }}=180^{\circ}\right)$.

(a) Straight Blade.

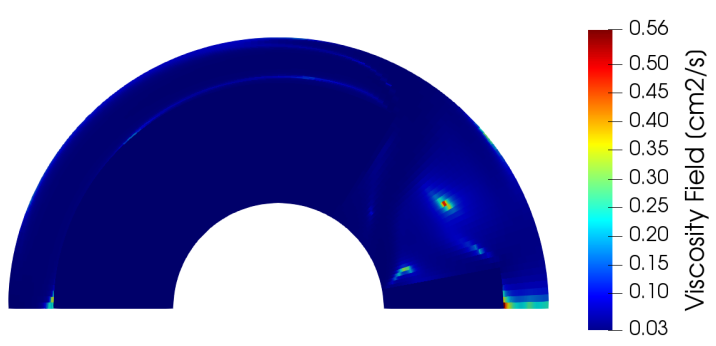

(c) Straight Blade $60^{\circ}$.

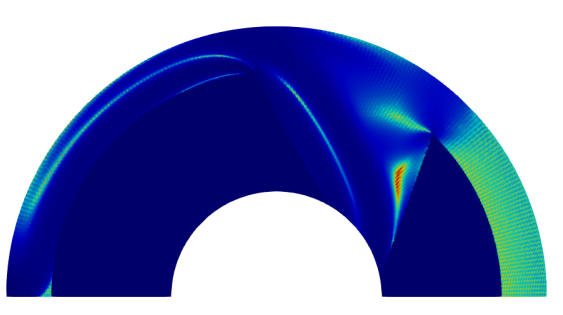

(b) Case 1 .

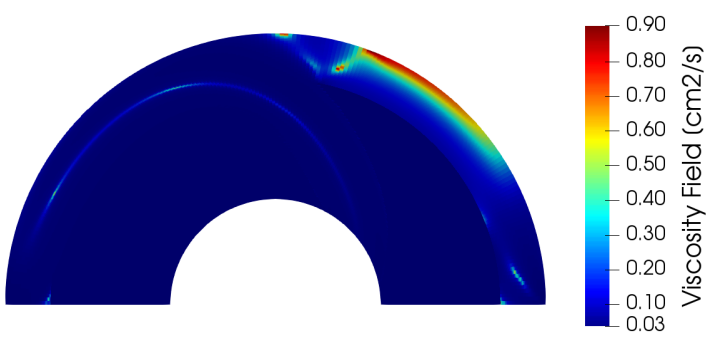

(d) Case 3 .
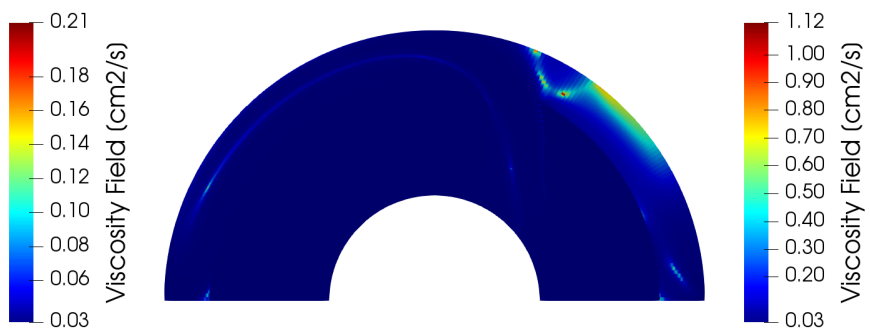

Figure 8.24 - Energy dissipation (point-wise) for Straight blade and Optimized topologies $\left(\beta_{\text {geo }}=180^{\circ}\right)$.

(a) Straight Blade.

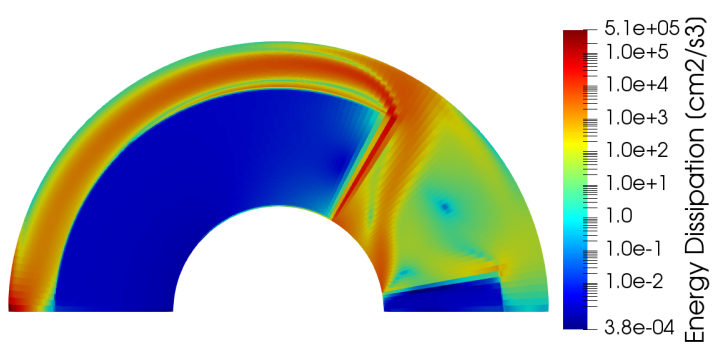

(c) Straight Blade $60^{\circ}$.

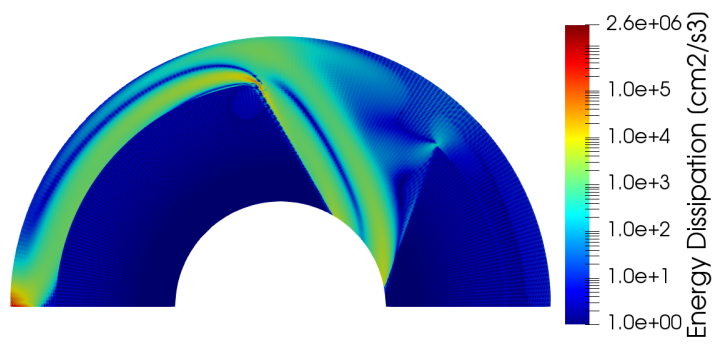

(b) Case 1 .

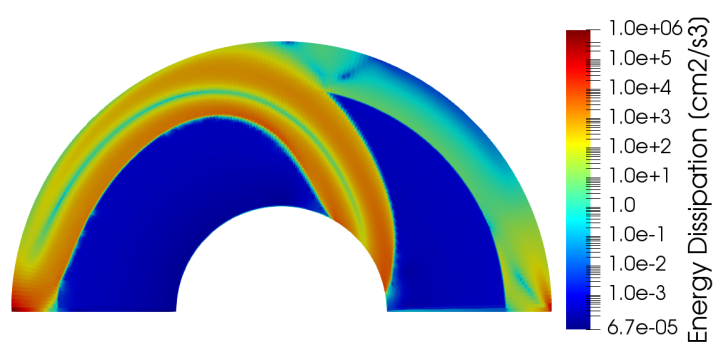

(d) Case 3 .

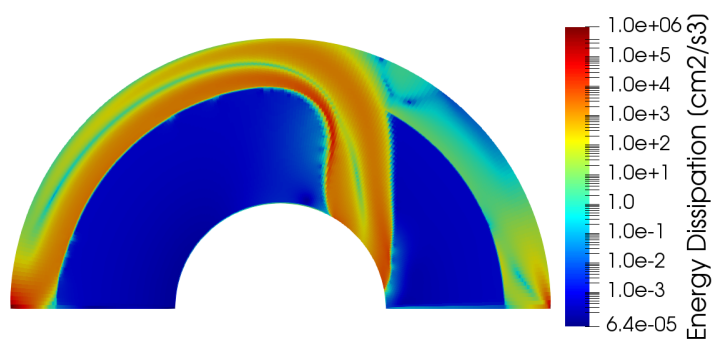

The energy dissipation and vorticity functionals can be analyzed with a point-wise plot, as shown in Figs. 8.24 and 8.25, respectively. The SB60 shows the same tendency present in the optimized topologies, in which the center of the flow has low values for both energy dissipation and vorticity. Comparing it with the optimized plots we can see that the optimized profile could be reached by reducing the blade area. However, the peak 
values for both functionals are still higher. From Tab. 8.12 shows that the SB60 is an intuitive shape to reduce the energy dissipation. Still, the optimized blades can reach even better values.

Figure 8.25 - Vorticity (point-wise) for Straight blade and Optimized topologies $\left(\beta_{\text {geo }}=180^{\circ}\right)$.

(a) Straight Blade.

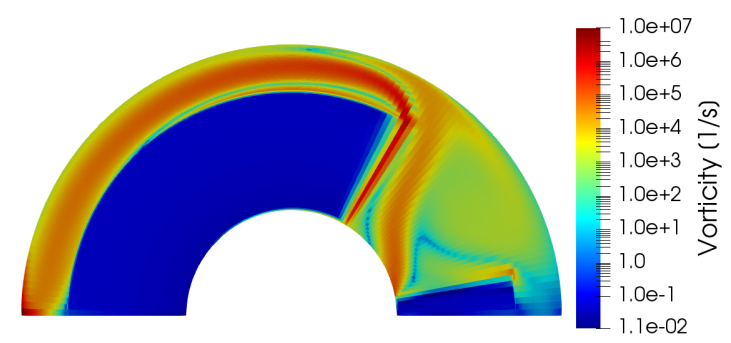

(c) Straight Blade $60^{\circ}$.

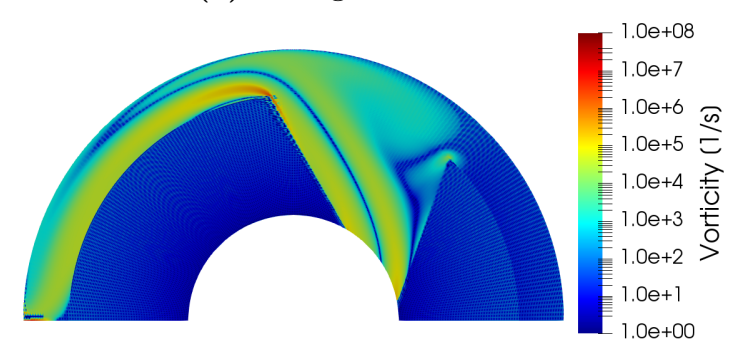

(b) Case 1.

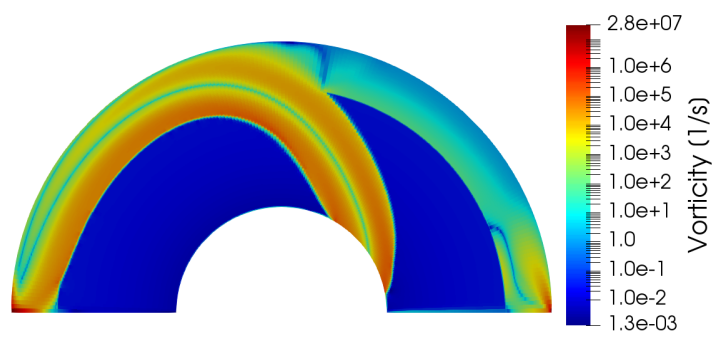

(d) Case 3 .

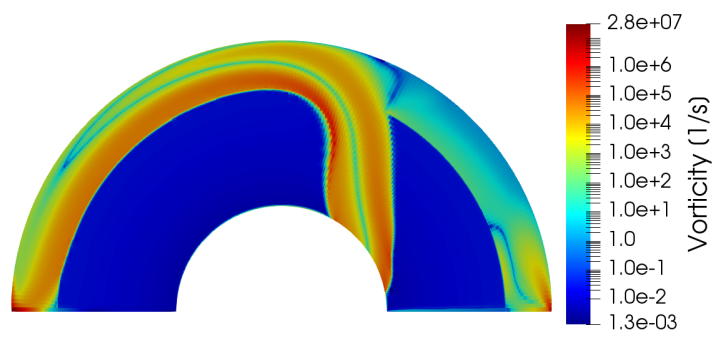

The isolated blades are shown in Fig. 8.26. Table 8.10 shows the functional values for the isolated blades. It is important to notice that the values are different from the previous sections because the new domain has a larger area of integration. The $\%$ column shows the value in relation to the straight blade. The optimized blades from Cases 1 (Fig. 8.26b) and 3 (Fig. 8.26d) maintain the energy dissipation values lower than the straight blade. The post-processing smooths the sharp edges in the SB60 transition region, causing a reduction in the functional value. This side effect makes the SB60 case have almost the same performance that the optimized blade from case 3, which indicates that case 3 is trapped at local minimum. Finally, the complete impellers are shown in Fig. 8.27, the blue regions in this figure represent the fluid and the blades are defined by the white portion.

Table 8.13 - Functional values of Isolated Straight and Optimized blades with $\beta_{\text {in }}=180^{\circ}$.

\begin{tabular}{lccccccc}
\hline & Straight Blade & SB60 & $\%$ & Case 1 & $\%$ & Case 3 & $\%$ \\
\hline Area & 2.30 & 2.30 & 0 & 2.18 & -5 & 2.00 & -13 \\
Mass Flow & 2.98 & 2.99 & 0 & 2.94 & -1 & 2.98 & 0 \\
Inlet Pressure $\left(\cdot 10^{5}\right)$ & -1.33 & -1.34 & 0 & -1.34 & 0 & -1.33 & 0 \\
Energy Dissip. $\left(\cdot 10^{3}\right)$ & 4.03 & 2.66 & -34 & 2.13 & -47 & 2.70 & -33 \\
Viscous Dissip. $\left(\cdot 10^{3}\right)$ & 4.03 & 2.66 & -34 & 2.13 & -47 & 2.70 & -33 \\
Friction Dissip. & 0.00 & 0.00 & 0 & 0.00 & 0 & 0.00 & 0 \\
Vorticity $\left(\cdot 10^{5}\right)$ & 0.94 & 0.60 & -36 & 0.37 & -60 & 0.62 & -34 \\
\hline
\end{tabular}


Figure 8.26 - Isolated Straight and Optimized blades for $\beta_{g e o}=180^{\circ}$.

(a) Straight Blade.

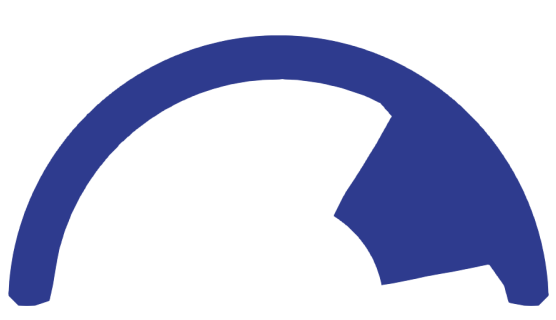

(c) Straight Blade $60^{\circ}$.

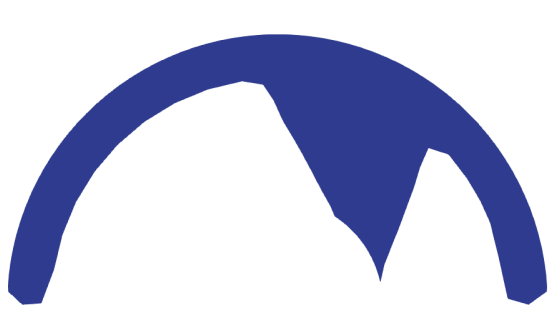

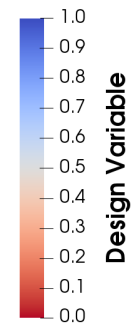

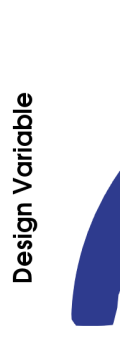

(b) Case 1 .

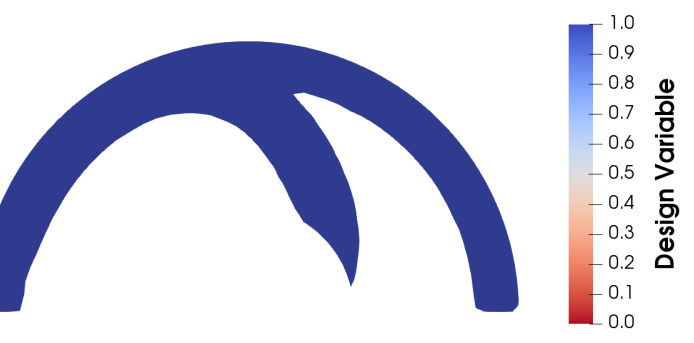

(d) Case 3 .

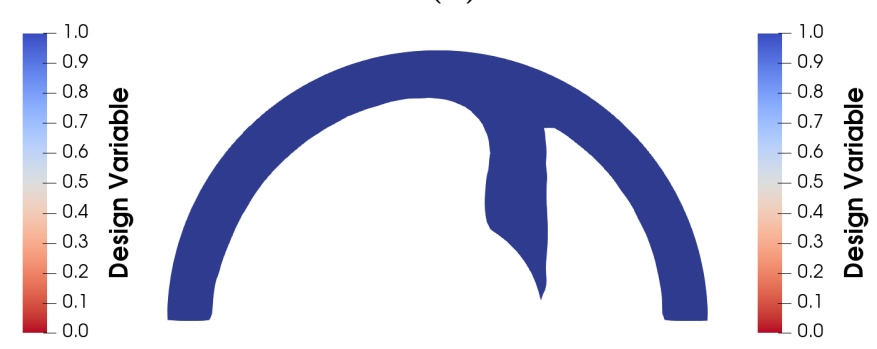

Figure 8.27 - Complete Impellers for Straight and Optimized blades $\left(\beta_{\text {geo }}=180^{\circ}\right)$.

(a) Straight Blade.

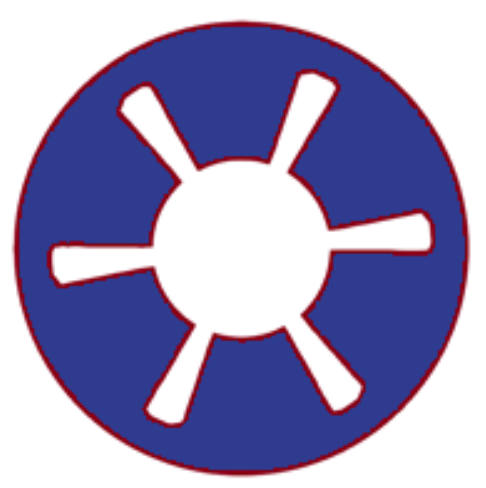

(c) Straight Blade $60^{\circ}$.

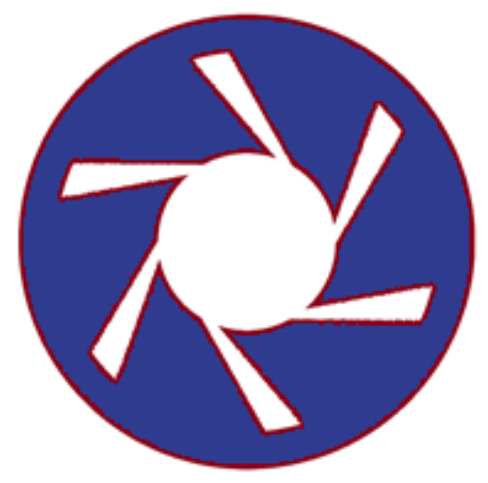

(b) Case 1.

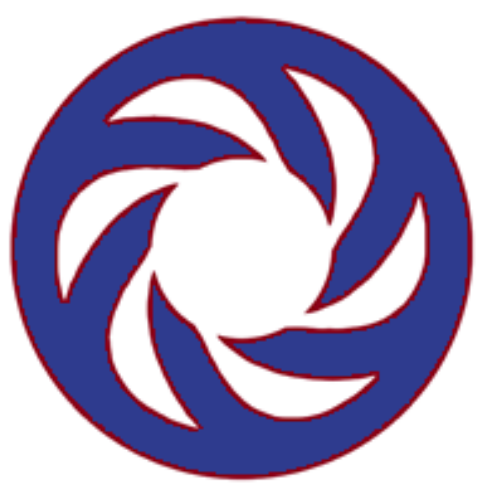

(d) Case 3 .

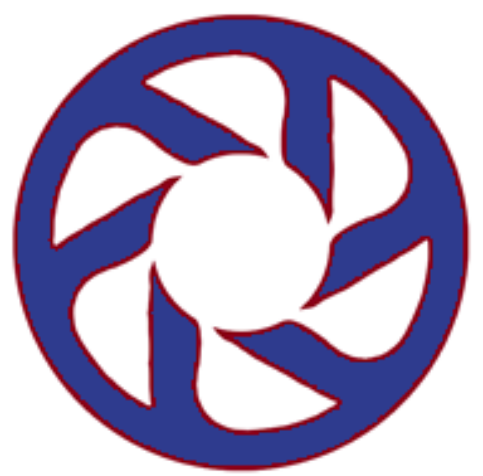




\subsection{Impeller Design (Non-Newtonian Turbulent Model)}

This section presents the model and results for the rotor design considering the Non-newtonian fluid coupled with the Spalart-Allmaras turbulence model. The domain is the same as the previous section, where a region at the external radius $\left(\Omega_{f i x}\right)$ is not optimized. The domain dimensions are inner radius of $7[\mathrm{~mm}]$, middle radius of $15[\mathrm{~mm}]$ $\left(\Omega_{\text {opt }}\right)$, and outer radius of $18[\mathrm{~mm}]$. Only one circular section is analyzed with $\beta_{\text {geo }}=120^{\circ}$, due to the results being not affected by the increase in radius. The boundary conditions are shown in Fig 8.28. The domain outlet has zero tension condition, for the RANS equations, and zero gradient at the normal direction for the Spalart-Allmaras equations. The walls have a no-slip condition (RANS eq.) and zero eddy viscosity (SA eq.). The inlet has an aperture of $\beta_{i n}=50^{\circ}$ with a parabolic velocity profile angled $\left(\theta_{u_{i n}}=60^{\circ}\right)$ in relation to the boundary normal direction, and constant eddy viscosity. A rotation of $3000[\mathrm{rpm}]$ and a inlet velocity $\left(u_{i n}\right)$ calculated to attend the mass flow of $3.0[l / \mathrm{min}]$ are used. The inlet viscosity for the Spalart-Allmaras model is calculated by:

$$
\begin{aligned}
\nu_{t_{i n}} & =\sqrt{\frac{3}{2}}\left\|\mathbf{u}_{\mathbf{i n}}\right\| I_{t} L_{t} \\
L_{t} & =0.07 \beta_{i n} r_{i n} \\
I_{t} & =0.05
\end{aligned}
$$

where $\left\|u_{i n}\right\|$ is the inlet velocity modulus, $L_{t}$ is the turbulence characteristic length, and $I_{t}$ is the turbulence intensity, here chosen as $5 \%$.

Figure 8.28 - Boundary conditions for the non-Newtonian turbulent impeller optimization.

$$
\begin{aligned}
& r_{\text {in }}=7 \mathrm{~mm} \\
& r_{o p t}=15 \mathrm{~mm} \\
& r_{o u t}=18 \mathrm{~mm} \\
& \theta_{u_{i n}}=60^{\circ} \\
& \begin{array}{l}
\omega=3000 \mathrm{rpm} \\
u_{\text {in }}=3 \mathrm{l} / \mathrm{min}
\end{array} \\
& v_{t_{\text {in }}}=\sqrt{\frac{3}{2} I_{t} L_{t}\left\|\boldsymbol{u}_{\text {in }}\right\|}
\end{aligned}
$$

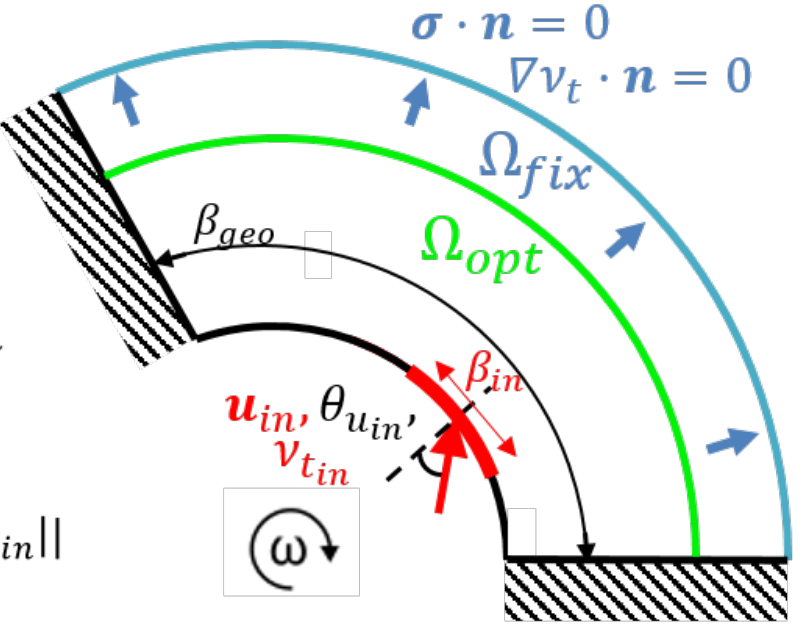


The previous sections show that the best topology is achieved with the optimization parameters from Case 1. The parameters used in this section for the forward problem is shown in Tab. 8.14 and the optimization constants are shown in Tab. 8.15. The optimization convergence curve is shown in Fig. 8.29 .

Table 8.14 - Inputs for Turbulent non-Newtonian Impeller forward Problem.

\begin{tabular}{lcc}
\hline Parameter & Value & Unit \\
\hline$r_{\text {in }}$ & 0.7 & {$[\mathrm{~cm}]$} \\
$r_{\text {opt }}$ & 1.5 & {$[\mathrm{~cm}]$} \\
$r_{\text {out }}$ & 1.8 & {$[\mathrm{~cm}]$} \\
$\beta_{\text {in }}$ & 50 & {$\left[{ }^{\circ}\right]$} \\
$\theta_{u_{\text {in }}}$ & 60 & {$\left[{ }^{\circ}\right]$} \\
$\rho$ & 1.06 & {$\left[\mathrm{~g} / \mathrm{cm}^{3}\right]$} \\
& & \\
$\mu(\dot{\gamma})$ & Carreau & $-\mathrm{Yasuda}$ \\
$\mu_{\infty}$ & 0.16 & {$[\mathrm{~Pa} \cdot \mathrm{s}]$} \\
$\mu_{0}$ & 0.0035 & {$[\mathrm{~Pa} \cdot \mathrm{s}]$} \\
$\lambda$ & 8.2 & \\
$a$ & 0.64 & \\
$n$ & 0.2128 & \\
& \multicolumn{3}{c}{} \\
$\left|u_{\text {in }}\right|$ & 39.79 & {$[\mathrm{~cm} / \mathrm{s}]$} \\
$\left|u_{\text {wall }}\right|$ & 0.0 & {$\left[\mathrm{~cm}^{2} \mathrm{~s}\right]$} \\
$\omega$ & 314.2 & {$[\mathrm{rad} / \mathrm{s}]$} \\
$\nu_{t_{\text {in }}}$ & 0.06 & {$\left[\mathrm{~cm}^{2} / \mathrm{s}\right]$} \\
$\nu_{t_{\text {wall }}}$ & 0.0 & {$\left[\mathrm{~cm}^{2} / \mathrm{s}\right]$} \\
\hline & &
\end{tabular}

Table 8.15 - Optimization parameters for non-Newtonian turbulent impeller optmization.

\begin{tabular}{lc}
\hline Parameter & Value \\
\hline Volume Constraint & $30 \%$ \\
Initial Distribution & 0.3 \\
$k_{\text {max }_{R A N S}}$ & 1,000 \\
$q_{R A N S}$ & $0.01-0.1$ \\
$k_{\text {max }_{S A}}$ & 10,000 \\
$q_{S A}$ & 0.1 \\
$k_{\text {max }}$ & 50 \\
$q_{\text {eik }}$ & 0.2 \\
\hline
\end{tabular}


Figure 8.29 - Convergence curve for non-Newt. Turbulent Impeller Optimization.

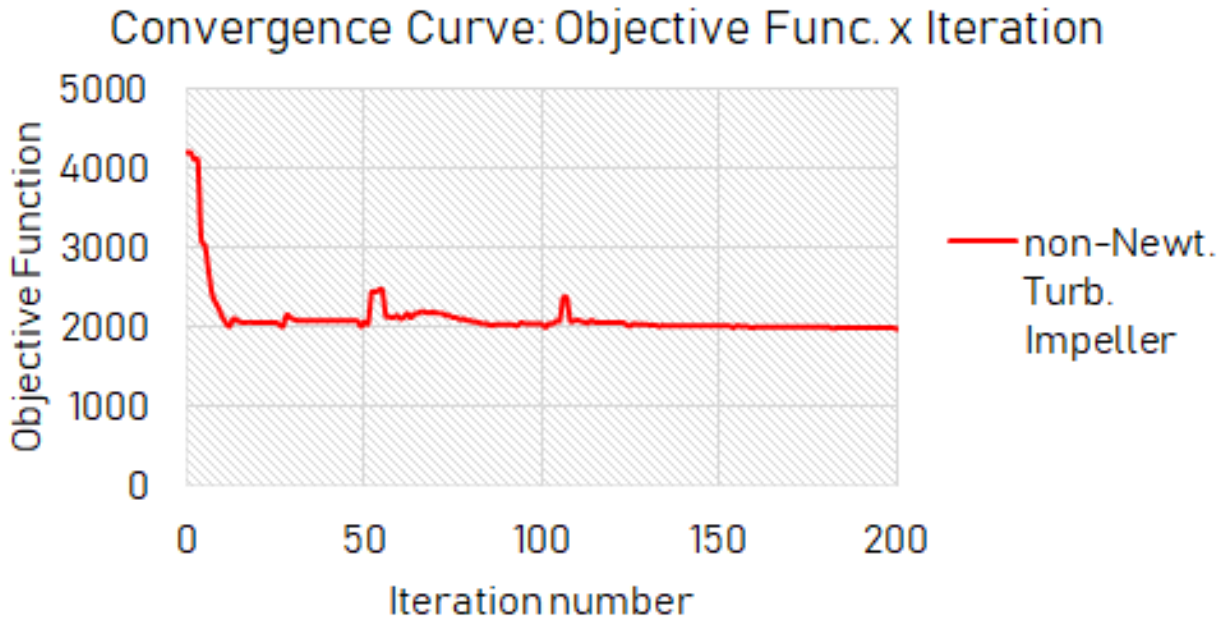

Figure 8.30 shows the final design and variable fields for the non-Newtonian Turbulent impeller optimization. The design is very similar to the Case 1 non-Newtonian result (Fig. $8.10 \mathrm{~b}$, which indicates that the turbulence model seems to have a small influence on this optimization. Also, it can be an effect of the small value for the turbulence inlet condition $(5 \%)$. Figure 8.31 shows a superposition of the designs. It can be seen that the designs are very similar, with small differences in the curvature of the blade left side. Hence, the optimization performed with only the non-Newtonian model should be enough to design optimized blades under the conditions presented here.

The velocity peak value $(74.8[\mathrm{~cm} / \mathrm{s}]$ from Fig. 8.30b) is lower than the value of the non-Newtonian optimization $(77.5[\mathrm{~cm} / \mathrm{s}]$ from Fig. $8.12 \mathrm{~b})$, which is an effect of the turbulence absorbing part of the energy. The pressure peak, however, is higher. The non-Newtonian viscosity plot (Fig. 8.30d) shows the same behavior as presented before; the highest value occurs near the center of the channel, where the velocity gradients are lower.

The Spalart-Allmaras eddy viscosity plot (Fig. 8.30e shows that most of the turbulence in the domain comes from the inlet, and a small portion is, in fact, produced in the domain. This is inferred from the fact that the highest value occurs at the inlet, and most of the distribution is a result of the convection from the inlet to the outlet. 
Figure 8.30 - Final Design for non-Newtonian turbulent impeller optimization.

(a) Optimized Blade.

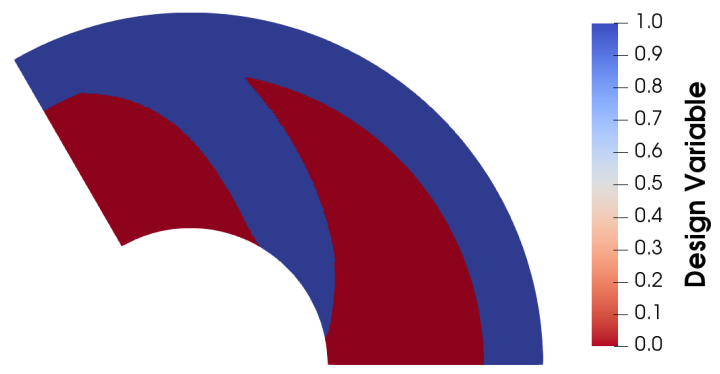

(b) Velocity Field.

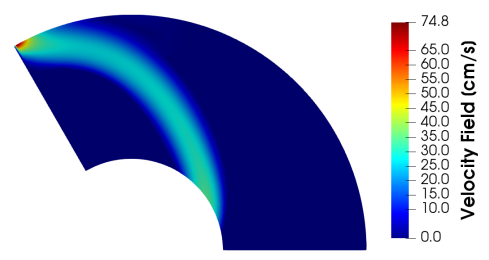

(e) S-A Eddy Viscosity $\nu_{t}$.

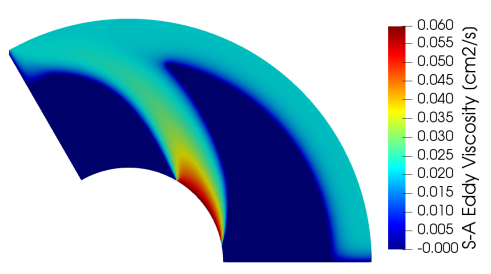

(c) Pressure Field.

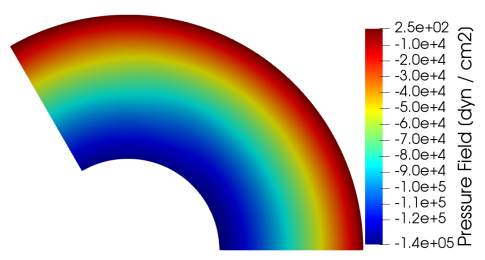

(f) Energy Dissipation.

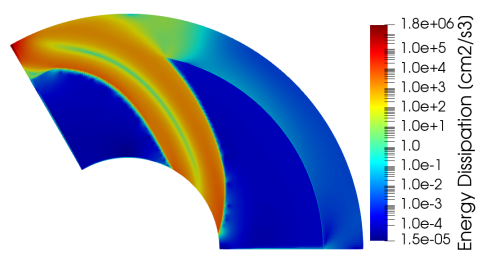

(d) non-Newtonian Viscosity.

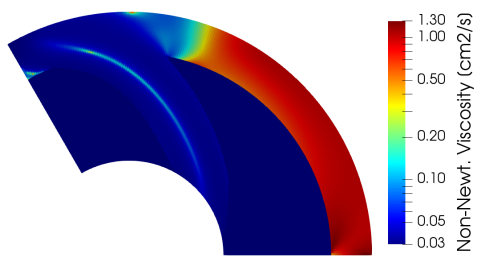

(g) Vorticity.

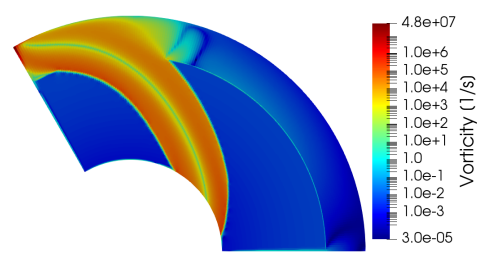

The energy dissipation (Fig.8.30f) and vorticity (Fig. 8.30g) present the same overall distribution as the non-Newtonian optimization, with small differences in the peak value. Also, it is noticeable that the energy dissipation and vorticity plots have very similar shapes, given that both are proportional to the velocity gradient. Thus, confirming the fact that optimizing the blade for energy dissipation is an indirect way to reduce vorticity as well. In addition, the scale shear stress is directly proportional to the energy dissipation, so the value would be calculated by dividing (point-wise) the energy dissipation by the sum of the non-Newtonian viscosity and the S-A eddy viscosity. Hence, given that both viscosities are lower than 1 , the value of the scalar shear stress would be higher than the value of the energy dissipation, however, presenting the same distribution. 
Figure 8.31 - Impeller design comparison between non-Newtonian laminar and non-N. turbulent models.

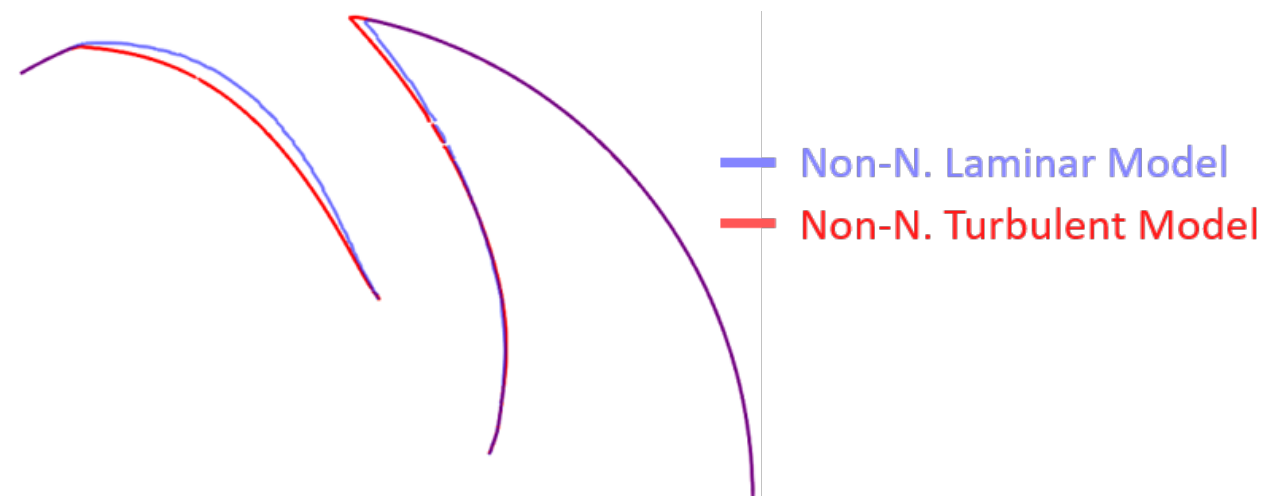

In order to verify the performance of the optimization, similarly to the previous sections, a straight blade impeller is defined. The variable plots for this domain with the non-Newtonian turbulence model are shown in Fig. 8.32. The plots are very similar to the ones of the previous section. The velocity field (Fig. 8.32b) shows a big recirculation area on the right side, while most of the flow velocity concentrates at the left side of the channel. The S-A eddy viscosity has the same behavior observed in the optimized blade, where the highest viscosity comes from the inlet is convected to the rest of the domain.

Figure 8.32 - Straight Blade variable distribution for non-Newtonian turbulent model.

(a) Straight Blade.

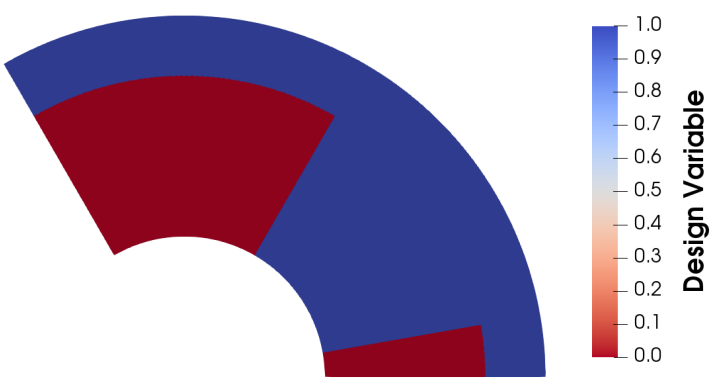

(b) Velocity Field.

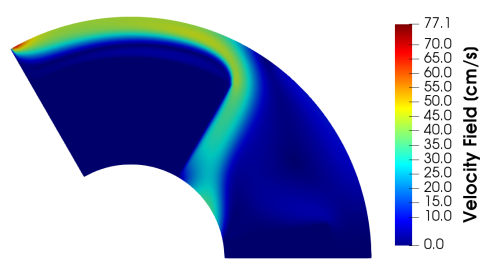

(e) S-A Eddy Viscosity $\nu_{t}$.

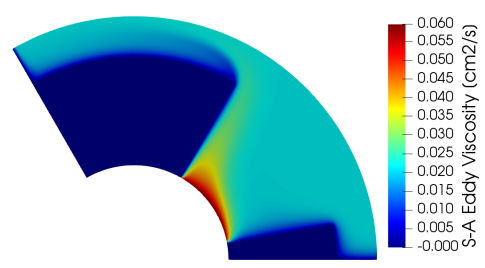

(c) Pressure Field.

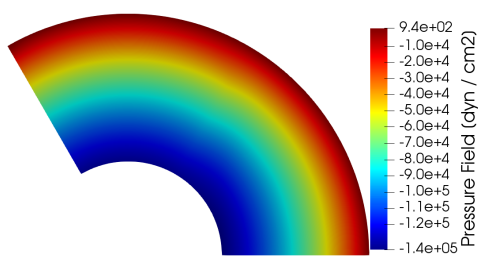

(f) Energy Dissipation.

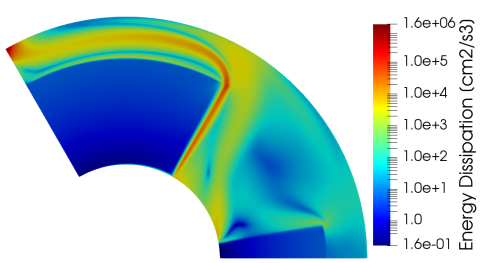

(d) non-Newtonian Viscosity.

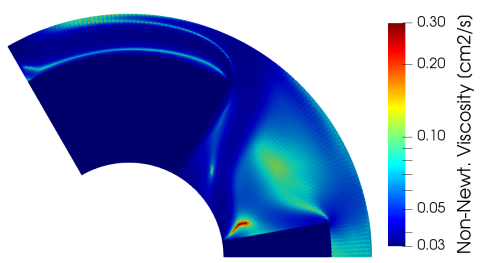

(g) Vorticity.

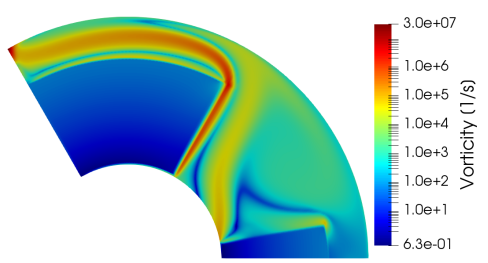


For the sake of completeness, Fig. 8.33 and Fig. 8.34 shows the post-processed design and variables for the optimized and straight blade cases, respectively. The variable distributions are very similar to the complete model (with material model).

Table 8.16 shows the functional values for both cases, with and without the material model. The post-processing causes a small area reduction in both blades. However, the mass flow entering the domain is almost the same. The optimized blade shows a Viscous Dissipation approximately $41 \%$ lower than the straight blade reference. Also, a reduction in the vorticity of approximately $54 \%$ is observed.

Figure 8.33 - Post-processed design for non-Newtonian turbulent impeller optimization.

(a) Optimized Blade.

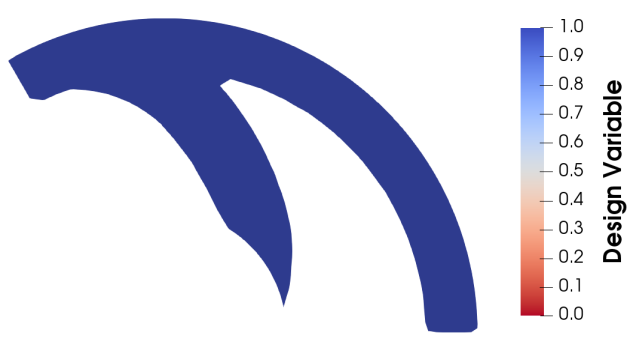

(b) Velocity Field.

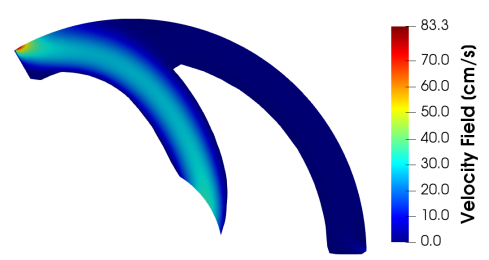

(e) S-A Eddy Viscosity $\nu_{t}$.

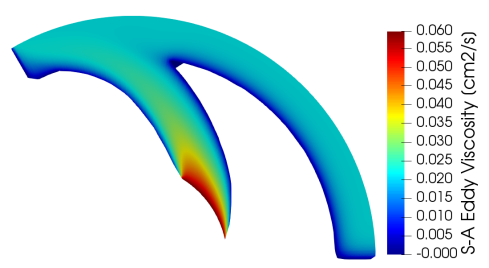

(c) Pressure Field.

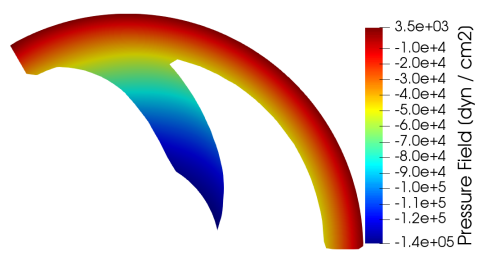

(f) Energy Dissipation.

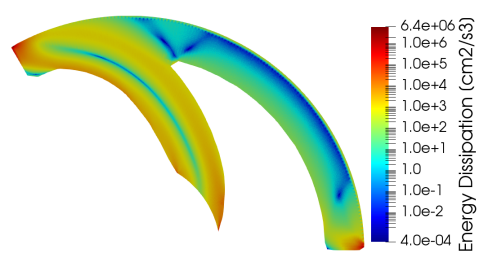

(d) non-Newtonian Viscosity.

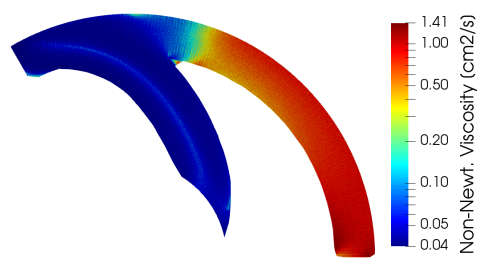

(g) Vorticity.

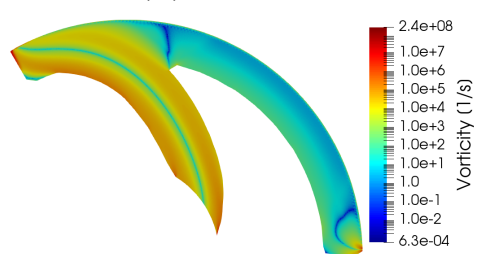


Figure 8.34 - Post-processed design for Straight Blade impeller with non-Newtonian turbulent model.

(a) Straight Blade.

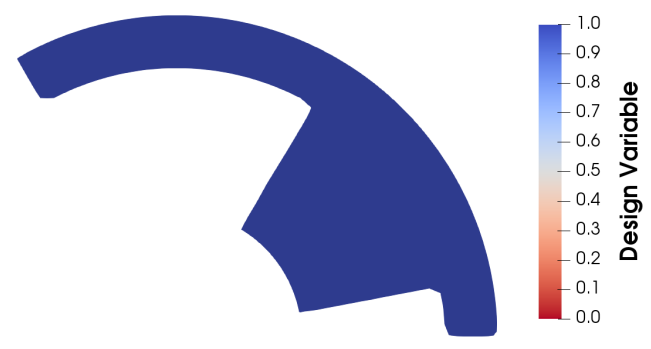

(b) Velocity Field.

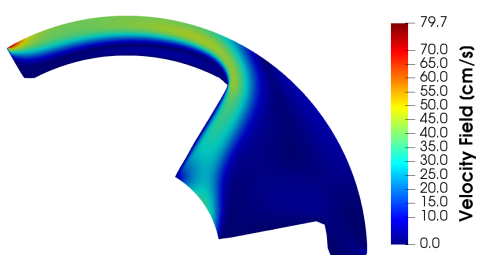

(e) S-A Eddy Viscosity $\nu_{t}$.

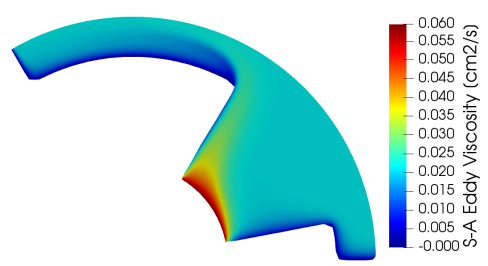

(c) Pressure Field.

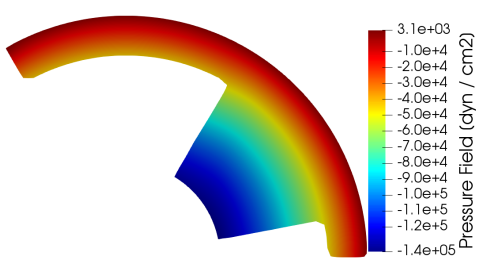

(f) Energy Dissipation.

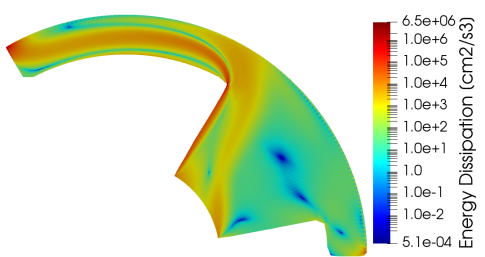

(d) non-Newtonian Viscosity.

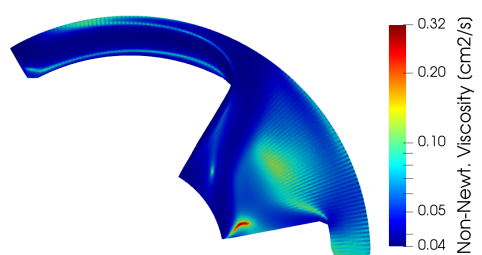

(g) Vorticity.

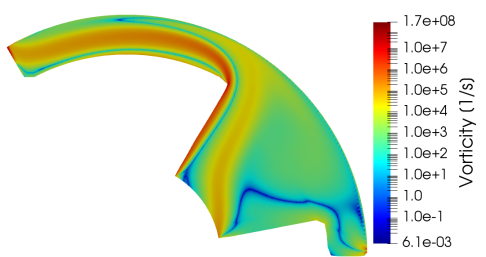

Table 8.16 - Functional values for non-Newtonian turbulent impeller optimization.

\begin{tabular}{lcccccc}
\hline & \multicolumn{3}{c}{ Complete } & \multicolumn{4}{c}{ Post-processed } \\
& SB & Opt. & $\%$ & SB & Opt. & $\%$ \\
\hline Area & 1.80 & 1.57 & -13 & 1.77 & 1.55 & -13 \\
Mass Flow & 2.99 & 2.99 & 0 & 2.98 & 2.96 & -1 \\
Inlet Pressure $\left(\cdot 10^{5}\right)$ & -1.3 & -1.3 & 0 & -1.3 & -1.3 & 0 \\
Energy Dissipation $\left(\cdot 10^{3}\right)$ & 3.5 & 2.0 & -44 & 3.5 & 2.1 & -41 \\
Viscous Dissipation $\left(\cdot 10^{3}\right)$ & 3.3 & 2.0 & -39 & 3.5 & 2.1 & -41 \\
Friction Dissipation & 263.6 & 12.7 & -95 & 0.0 & 0.0 & - \\
Vorticity $\left(\cdot 10^{4}\right)$ & 7.3 & 4.2 & -43 & 7.7 & 3.5 & -54 \\
\hline
\end{tabular}




\subsection{Volute Design}

The works in literature that consider the volute design usually focus on industrial equipment. Most of the works considering the optimization are performed on compressors (HEINRICH; SCHWARZE, 2016) and centrifugal blowers (BALONI; PATHAK; CHANNIWALA, 2015). A more recent work (GHADIMI et al., 2018) performed the volute optimization for a blood pump by considering a shape optimization coupled with a genetic algorithm. However, to the best of the author's knowledge, no work dealing specifically with the topology optimization of volutes has been reported. It is important to notice that the formulation used here does not consider a rotational domain, and the rotation given by the rotor is abstracted as an inclination of the inlet velocity. Thus, the same equations presented for the rotational frame are used by considering a null rotational velocity $(\omega=0)$.

The studied case considers an impeller with $30[\mathrm{~mm}]$ diameter. The domain dimensions and the boundary conditions are shown in Fig $8.35 \mathrm{a}$ and Fig $8.35 \mathrm{~b}$, respectively. The non-Newtonian Carreau-Yasuda model is used. The domain outlet has a zero tension condition, and the flows can only leave the domain in the normal direction of the boundary $\left(u_{x}=0\right)$. The walls have a no-slip condition, and the inlet has a velocity with a direction defined by the angle $\theta$ in relation to the boundary normal direction. Considering the impeller results from the previous sections the inlet velocity angle used is $\theta=85^{\circ}$

Figure 8.35 - Domain dimensions and boundary conditions for the volute optimization..

(a) Domain Dimension.

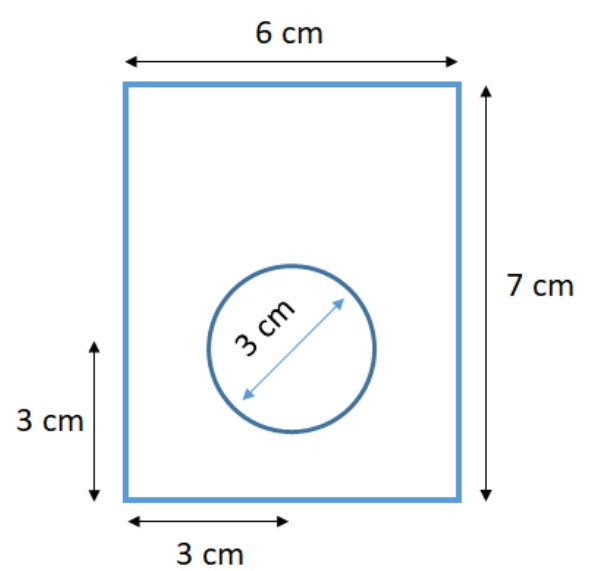

(b) Boundary Conditions.

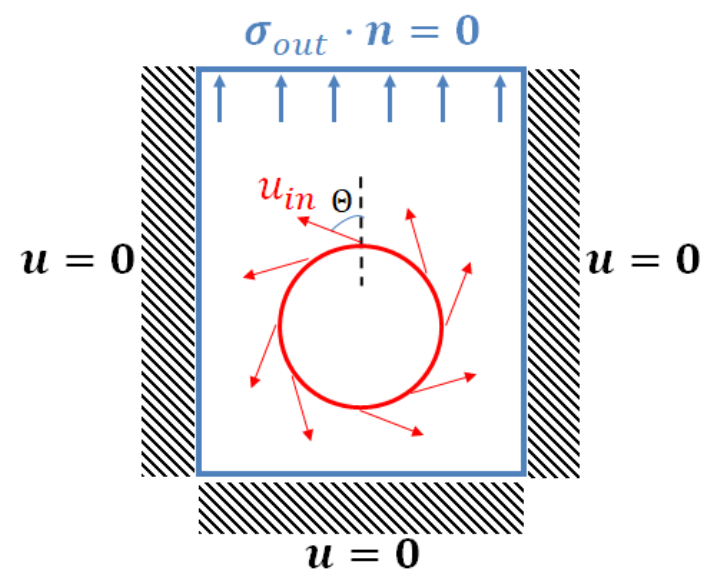




\subsubsection{Volute Design (Non-Newtonian Model)}

The results presented here consider a laminar flow, i.e., the turbulence model is not used. The topology and variable fields (velocity, pressure, viscosity, energy dissipation, and vorticity) are shown in Fig. 8.37. The convergence of the objective function for this case is shown in Fig. 8.36 .

Figure 8.36 - Convergence curve for non-Newtonian Volute Optimization.

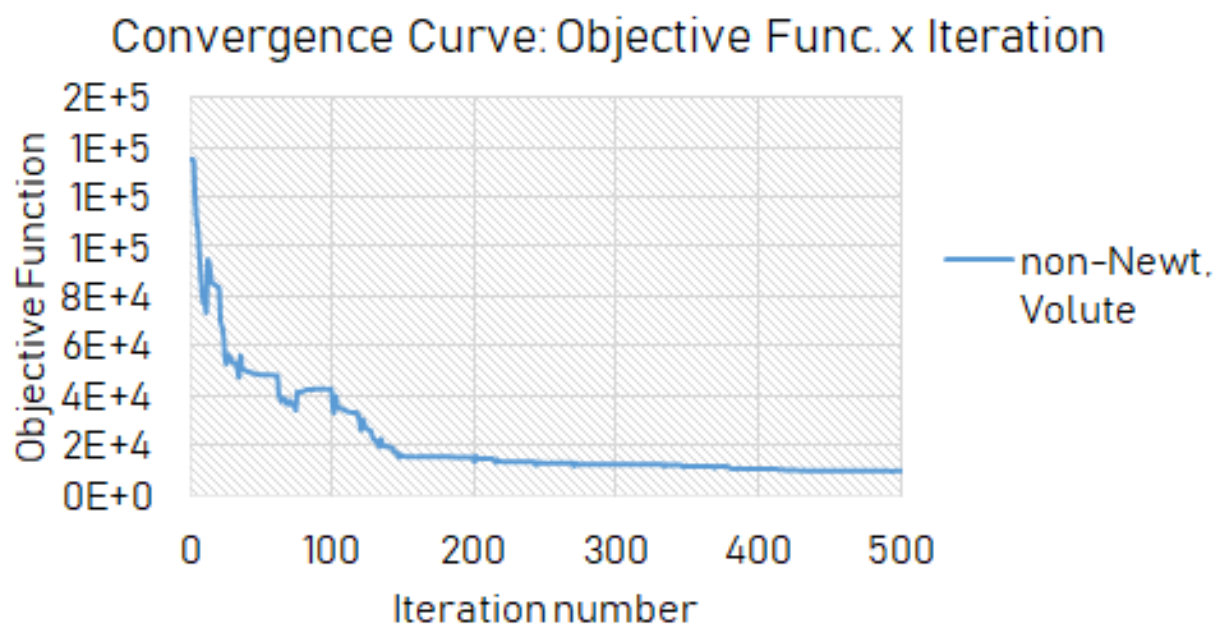

The velocity distribution (Fig. 8.37b) shows that the algorithm tries to maintain the velocity constant as the flow enters the domain. This is done by increasing the transverse area to accommodate the velocity input from the impeller. This is the same tendency presented in the traditional designs (STEPANOFF, 1993).

The pressure distribution (Fig. 8.37c) shows that the pressure is present on the solid material. However, its effect on the solid region is not important, given that only the pressure inside the fluid domain has a real effect on the flow.

The viscosity field shows that at the center of the channel the viscosity is higher, due to the lower velocity gradients there. While, near the walls, the gradients are higher and so the viscosity is lower, respecting the Carreau-Yasuda model. The same behavior is observed at the energy dissipation and vorticity point-wise plots (Figs. 8.37e and 8.37f, respectively), where, the values are higher near the walls due to the highest gradients. Even though the highest gradient occurs near the right side of the flow, it decreases rapidly near the center of the channel. This is a reflex to the velocity profile, which tries to maintain the velocity constant in most of the channel. 
Figure 8.37 - Optimized non-Newtonian Volute topology and fields.

(a) Topology.

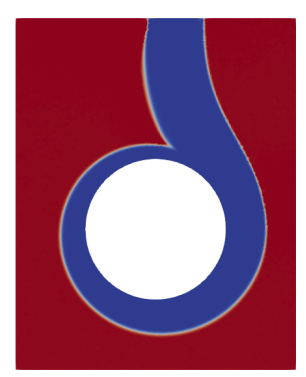

(d) non-Newtonian Viscosity.

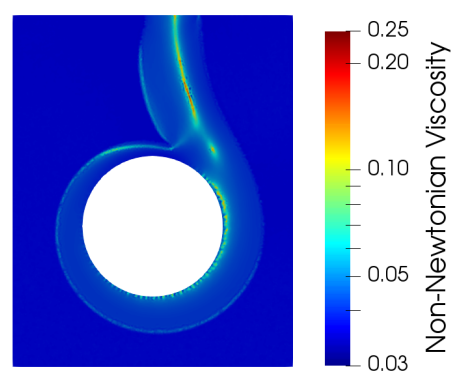

(b) Velocity.
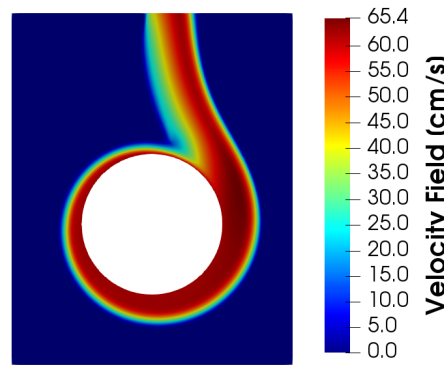

(e) Energy Dissipation.

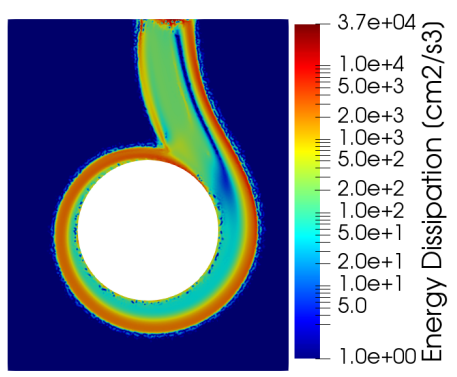

(c) Pressure.

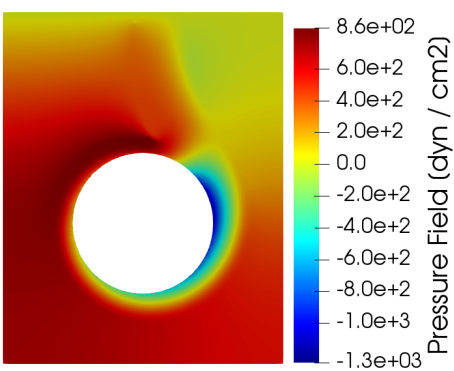

(f) Vorticity.

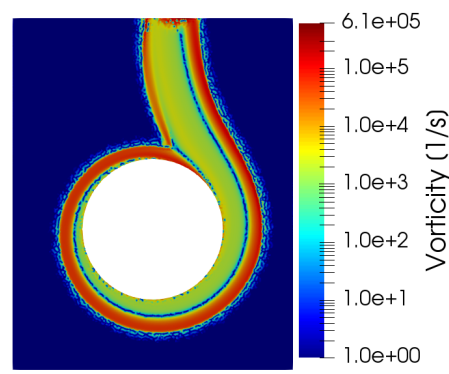

In order to verify the material model influence in the problem, the topology is isolated and post-processed. The topology and variables fields for this case are shown in Fig. 8.38. As we can see, most of the variable distributions are very similar to the complete model (Fig. 8.37), except for the viscosity, energy dissipation, and vorticity, which are all proportional to the velocity gradient. This indicates that these gradients increase with the imposition of the zero velocity at the walls. However, the distribution patterns are still very similar, and the main change is at the peak value of the fields.

Table 8.17 shows the functional values for both the complete model with material model and the post-processed topology. The post-processing reduced the topology area by $3 \%$. The pressure integral at the inlet has almost doubled. However, the mass flow, viscous dissipation, and vorticity values are very close. This indicates that the velocity fields are very similar. Hence, the material model can emulate well the wall boundary condition. 
Table 8.17 - Functional values of Complete and Isolated Optimized non-Newtonian Volute.

\begin{tabular}{lccc}
\hline & With Material Model & Post-processed & $\%$ \\
\hline Area $\left(\mathrm{cm}^{2}\right)$ & 11.38 & 11.04 & $-3.0 \%$ \\
Mass Flow $(\mathrm{L} / \mathrm{min})$ & 3.01 & 2.99 & $-0.7 \%$ \\
Inlet Pressure $\left(\cdot 10^{2}\right)$ & -2.3 & -4.6 & $100.2 \%$ \\
Energy Dissipation $\left(\cdot 10^{4}\right)$ & 1.9 & 1.0 & $-44.5 \%$ \\
Viscous Dissipation $\left(\cdot 10^{4}\right)$ & 1.0 & 1.0 & $2.2 \%$ \\
Friction Dissipation $\left(\cdot 10^{4}\right)$ & 8.5 & - & - \\
Vorticity $\left(\cdot 10^{5}\right)$ & 2.0 & 2.1 & $3.9 \%$ \\
\hline
\end{tabular}

Figure 8.38 - Post-processed Optimized non-Newtonian volute topology and fields.

(a) Topology.

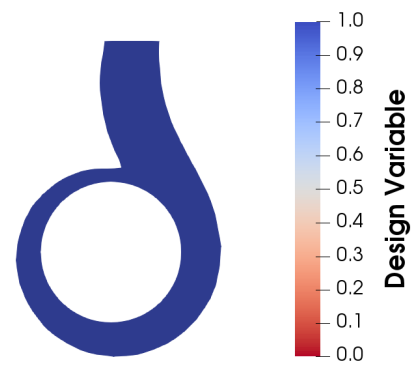

(d) non-Newtonian Viscosity.

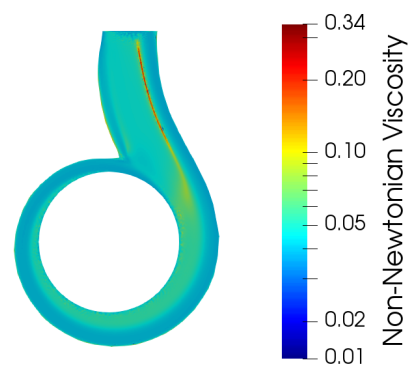

(b) Velocity.

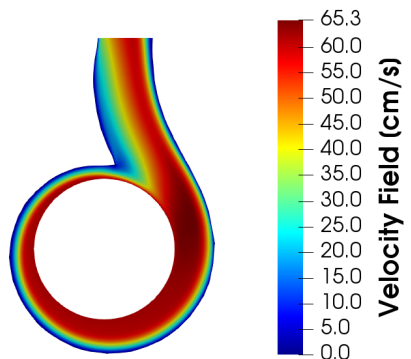

(e) Energy Dissipation.

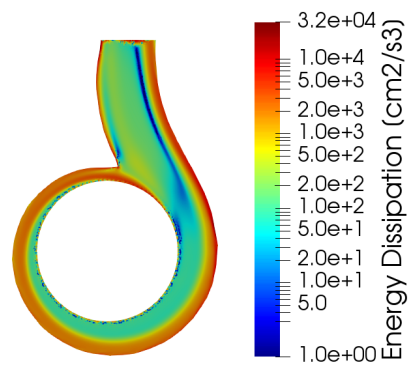

(c) Pressure.

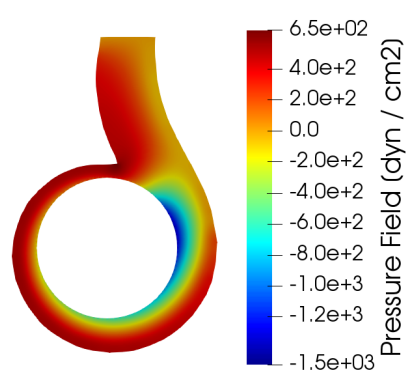

(f) Vorticity.

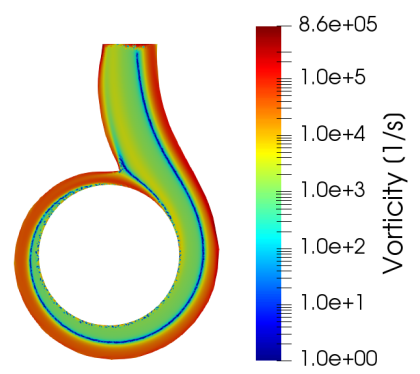

\subsubsection{Volute Design (Non-Newtonian Turbulent Model)}

The results presented here consider non-Newtonian turbulent model, i.e., the Carreau-Yasuda viscosity model coupled with the Spalart-Allmaras model. The domain dimensions are kept the same (Fig. 8.35), and the boundary conditions are changed to address the turbulence model, as shown in Fig. 8.39. The inlet has a constant eddy viscosity $\left(\nu_{t}\right)$ calculated by Eq. 8.1. The walls have an eddy viscosity value equal to zero, and the outlet has the zero gradient at normal direction equal to zero. 
Figure 8.39 - Boundary conditions for the non-Newtonian turbulent volute optimization..

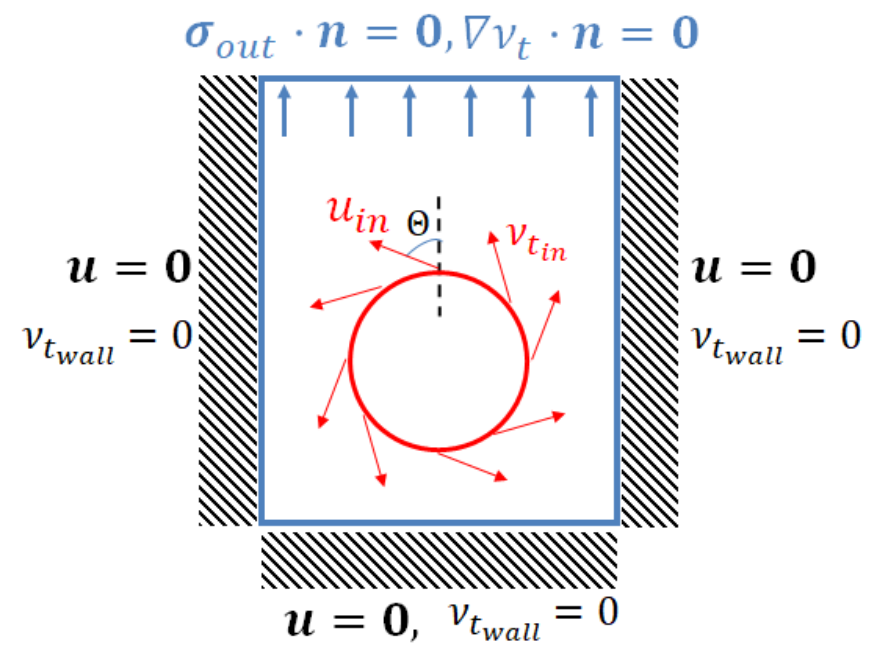

The final topology and variable distributions for this case are shown in Fig. 8.41. The topology is not very discrete and presents regions with intermediary values between 0 and 1. Hence, this topology needs to be post-processed so that the result can have physical meaning. The post-processed topology can be seen in Fig. 8.42. The convergence of the objective function for this case is shown in Fig. 8.40.

Figure 8.40 - Convergence curve for non-Newtonian Turbulent Volute Optimization.

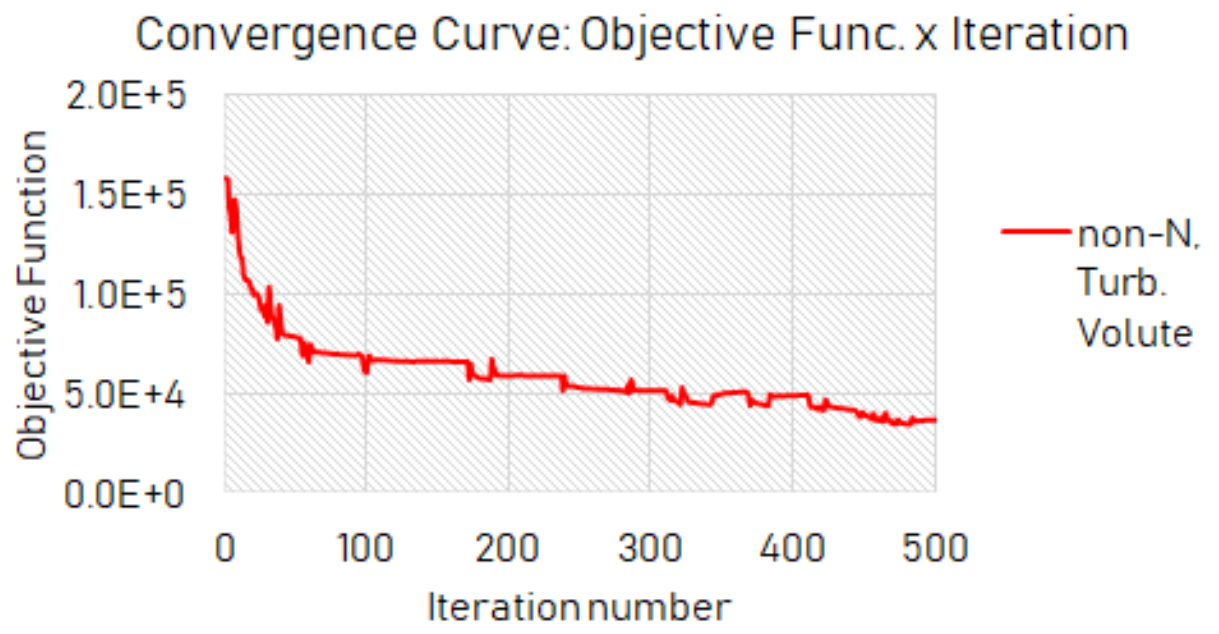

Comparing the variable fields of the complete case with the post-processed case, we can see that they show good agreement. The non-Newtonian viscosity plots (Figs. 8.41d and $8.42 \mathrm{~d}$ ) have some differences that are caused by the gray areas, given that the material model acts on the non-Newtonian model by Eq. 5.2 reducing the viscosity at regions with intermediary porosity. This same effect is seen at the S-A eddy viscosity plot (Figs. 8.41e and $8.42 \mathrm{e}$. Both viscosities influence the velocity distribution and its gradients directly, thus, the energy dissipation and vorticity plots are not exactly equal between the original and post-processed topologies. 
Figure 8.41 - Optimized non-Newtonian turbulent volute topology and fields.

(a) Topology.

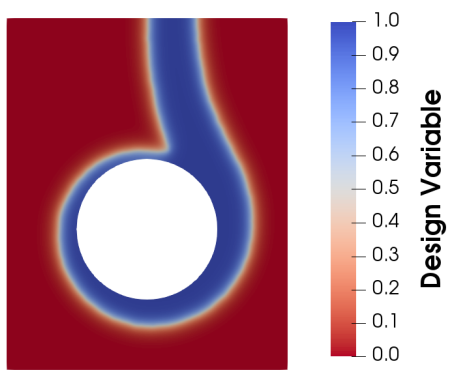

(b) Velocity.
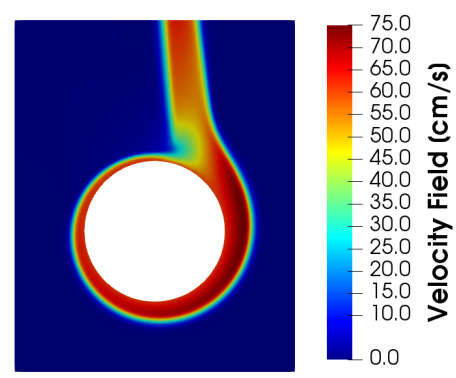

(e) S-A Eddy Viscosity $\left(\nu_{t}\right)$.

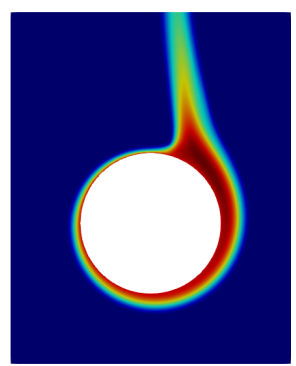

(c) Pressure.

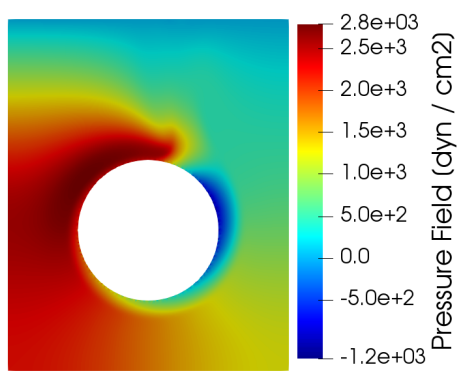

(f) Energy Dissipation.

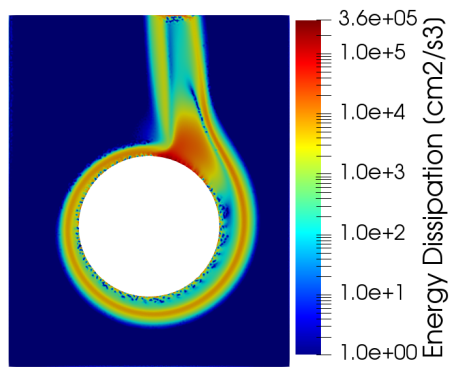

(d) non-Newtonian Viscosity.

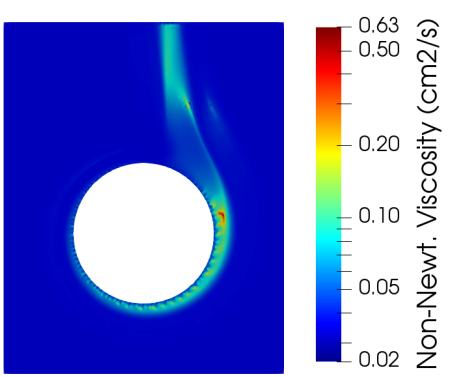

(g) Vorticity.

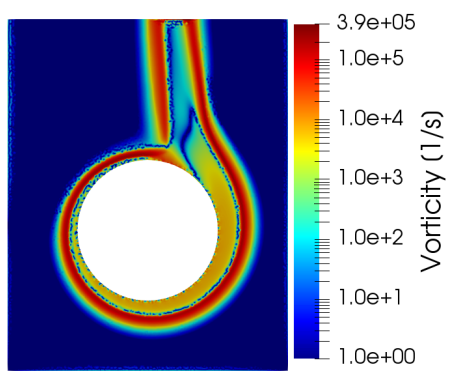


Figure 8.42 - Post-processed topology and fields of non-Newtonian turbulent volute optimization.

(a) Topology.

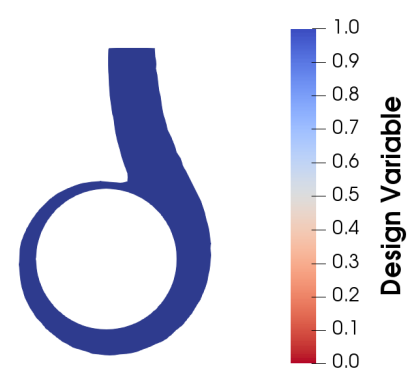

(b) Velocity.

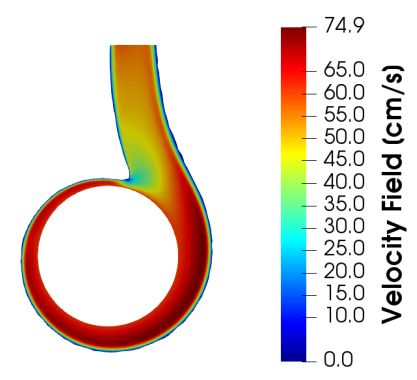

(e) S-A Eddy Viscosity $\left(\nu_{t}\right)$.

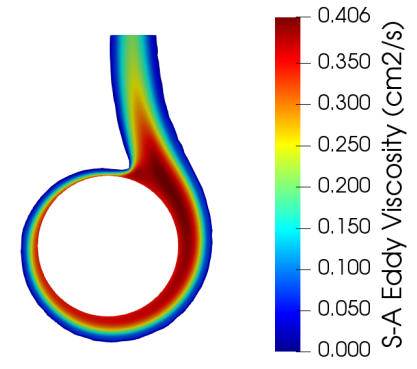

(c) Pressure.

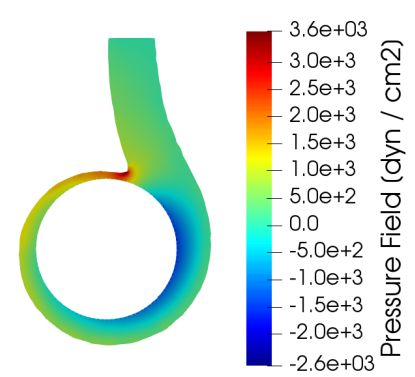

(f) Energy Dissipation.

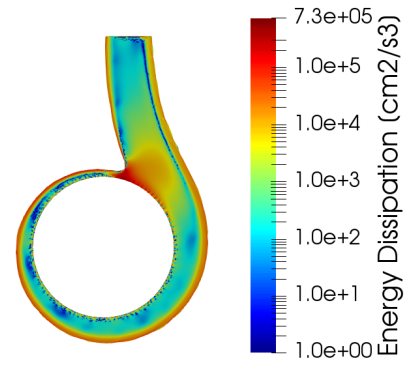

(d) non-Newtonian Viscosity.

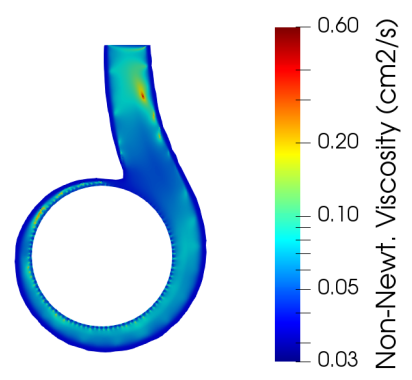

(g) Vorticity.

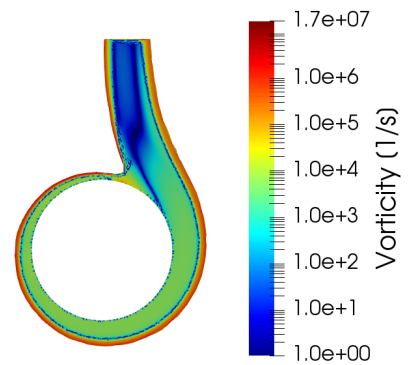

Table 8.18 shows the functional values for the complete model and the post-processed result for the non-Newtonian turbulent volute optimization. The area and mass flow are very similar. The pressure integral is very different because the pressure distribution in the complete model (Fig. 8.41c) has a large high-pressure area at the solid region on the left side of the inlet, that affects the inlet pressure. The gray regions cause a high friction dissipation in the complete model. It also causes a reduction of viscous dissipation and vorticity (reducing the velocity gradients), which are lower in the complete model $\left(3.7 \cdot 10^{4}\right.$ and $\left.2.6 \cdot 10^{5}\right)$ when compared to the post-processed case $\left(6.6 \cdot 10^{4}\right.$ and $\left.7.4 \cdot 10^{5}\right)$. However, the gray regions do not have physical meaning and can not be easily built, so the post-processed topology is the real result. 
Table 8.18 - Functional values of Complete and Isolated Optimized non-Newtonian turbulent volute.

\begin{tabular}{lccc}
\hline & With Material Model & Post-processed & $\%$ \\
\hline Area $\left(\mathrm{cm}^{2}\right)$ & 8.98 & 8.64 & $-3.8 \%$ \\
Mass Flow $(\mathrm{L} / \mathrm{min})$ & 3.00 & 3.02 & $0.8 \%$ \\
Inlet Pressure $\left(\cdot 10^{2}\right)$ & 9.8 & -2.1 & $-121.1 \%$ \\
Energy Dissipation $\left(\cdot 10^{4}\right)$. & 12.4 & 6.6 & $-46.6 \%$ \\
Viscous Dissipation $\left(\cdot 10^{4}\right)$ & 3.7 & 6.6 & $78.0 \%$ \\
Friction Dissipation $\left(\cdot 10^{4}\right)$ & 8.7 & - & - \\
Vorticity $\left(\cdot 10^{5}\right)$ & 2.6 & 7.4 & $191.5 \%$ \\
\hline
\end{tabular}

Figure 8.43 shows a superposition comparison between the post-processed topology obtained with the non-Newtonian turbulent model (Fig. 8.42a) and the topology obtained with the laminar non-Newtonian model (Fig. 8.37a). As we can see, overall, the curvatures are very similar for most regions of the volutes, and the main differences occur at the outlet channel. The turbulence model seems to promote a straighter channel than the laminar case, what can be explained by the higher inertia caused by the additional viscosity introduced by the RANS model, in which the energy of the fluctuating velocities of small scale eddies are interpreted as the eddy viscosity $\left(\mu_{t}\right)$.

Comparing the post-processed non-Newtonian viscosity plots of the non-Newtonian turbulent case (Fig. 8.42d) with the plot of the laminar case (Fig. 8.38d) we can see that the turbulent case has a higher peak value, indicating that the velocity gradients (and shear rate) are lower. However, when we consider the turbulence, there are new velocity gradients that arise from the eddies and increase the viscous dissipation by increasing the eddy viscosity $\left(\mu_{t}\right)$, as can be seen in Eq. 5.13. Thus, the peak of the energy dissipation plot for the turbulent case (Fig. 8.42f) is higher than the one from the laminar case (Fig. $8.38 \mathrm{e}$.

Figure 8.43 - Volute design comparison between non-Newtonian and non-N. turbulent models.

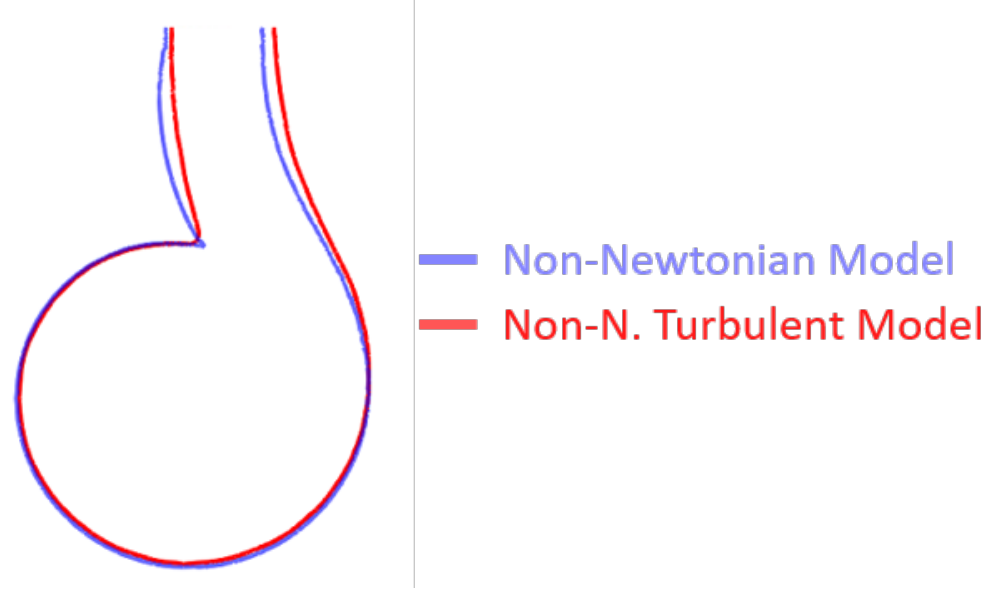




\section{EXPERIMENTAL METHODOLOGY}

This chapter presents the prototype design description, the manufacturing process, and the experiment description. Two prototypes, with different drivers, are built. The first had a slide bearing and considered optimized impellers for water (Newtonian fluid) and laminar flow and is published in the work Sá et al. (2018). The second considers the magnetic levitation for the bearing and uses the optimized topologies obtained in this work for blood (non-Newtonian fluid) and turbulent flow. The following sections describe this last prototype.

The VAD characterization is made by measuring the pump output (pressure, at the inlet and the outlet, and mass flow).

\subsection{Pump Prototype}

The prototype consists, essentially, of three parts: the volute casing, the driver, and the rotor. The driver consists of a traditional brushless electric motor that rotates a wheel of magnets and promotes the impeller motion without contact. The rotation control is done by manually adjusting the DC source output. A $3 \mathrm{~mm}$ aluminum disc is added to the bottom of the impeller to create the magnetic pair of the driver. This disc is dragged with the wheel of magnets by the principle of parasite current. Figure 9.1 shows the driver assembly.

Figure 9.1 - Driver assembly.

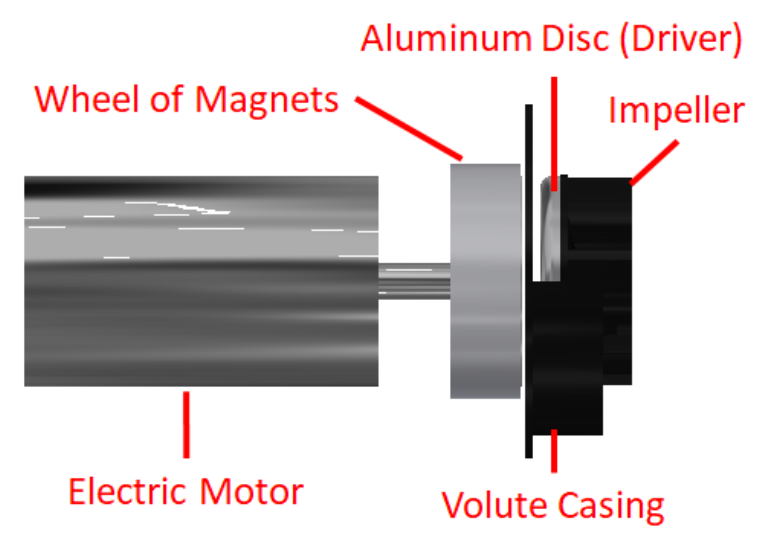

The bearing is a combination of hydraulic bearings and magnetic levitation. The hydraulic bearing is formed by a thin layer of fluid that appear when the impeller is rotating. This layer needs a sizeable superficial area to maintain the distance between the impeller and the casing, thus, at the top of the impeller, a thin aluminum disc of 1 
$m m$ is added, while, at the bottom, the aluminum disc of the driver serves as the other surface. A pair of magnets promote the magnetic levitation, one cylindrical magnet fixed at the center of the volute casing and one circular crown magnet fixed at the center of the rotor. This pair gives radial support and acts as a spring in this direction. Figure 9.2 shows an exploded view of the complete prototype where all the parts mentioned above are identified.

Figure 9.2 - Prototype assembly.

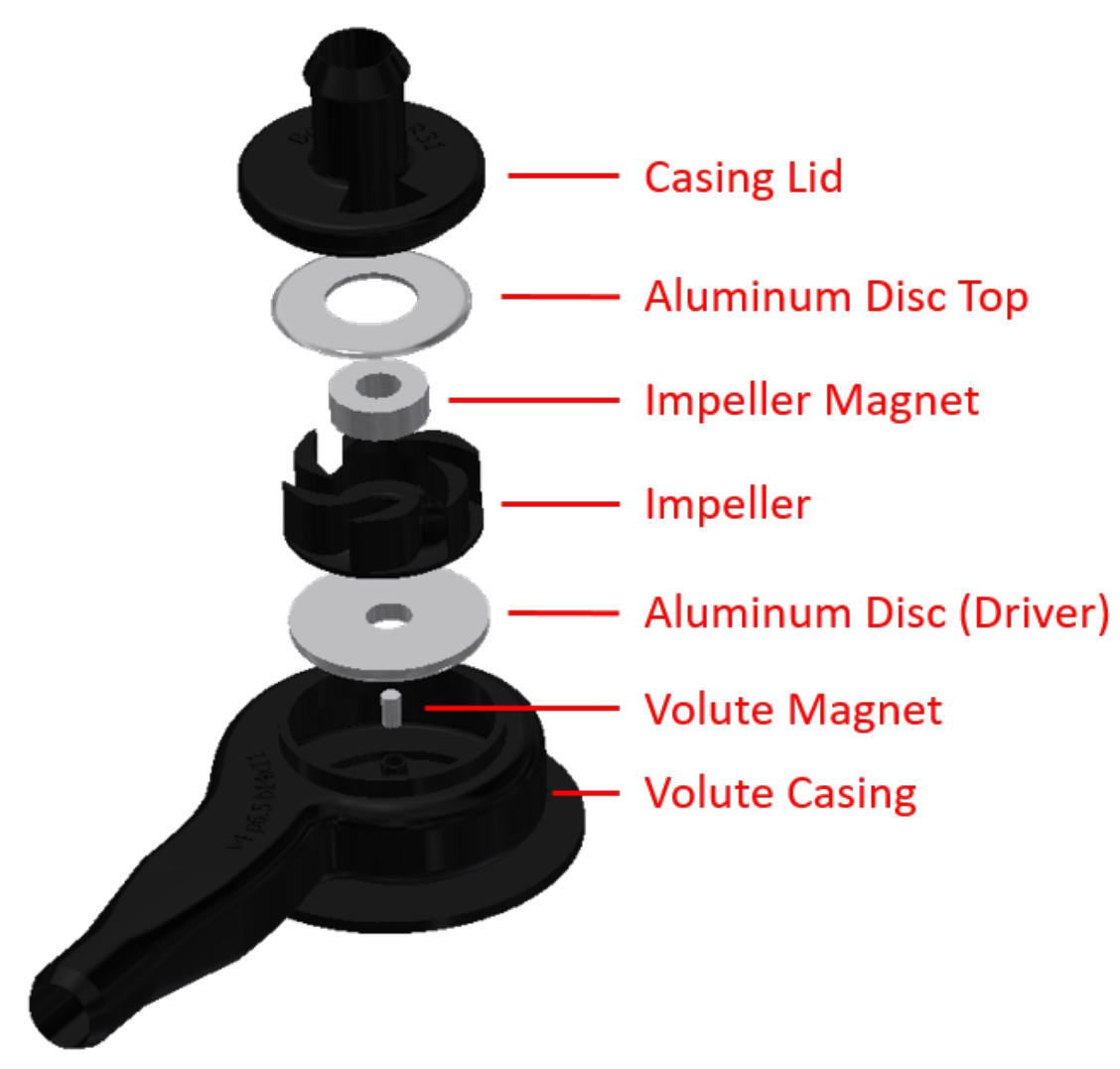

\subsubsection{D Printed Parts}

The prototype volute casing, lid, and rotors are built by using the 3D printer Objet30. The material used is the Stratasys commercial material VeroBlack, which offers good dimensional stability, is rigid, and opaque. The volute casing, lid, and impeller are shown in Figure 9.3 . The heights of the impeller and volute are $10[\mathrm{~mm}]$ and $18[\mathrm{~mm}]$, respectively. 
Figure 9.3 - Picture of the 3D printed parts.

(a) Opt. Volute Casing.

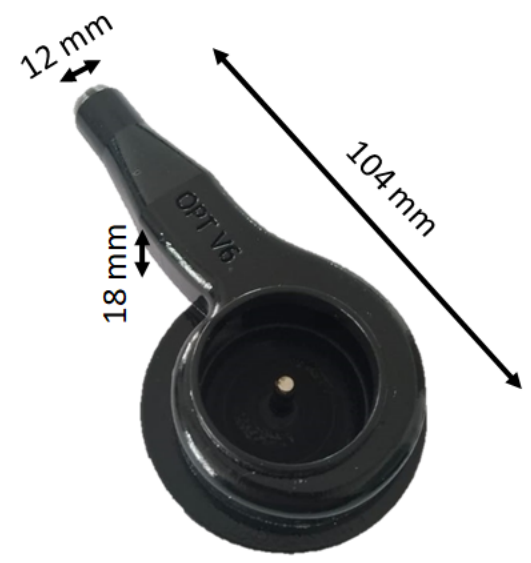

(b) Lid (Inlet).

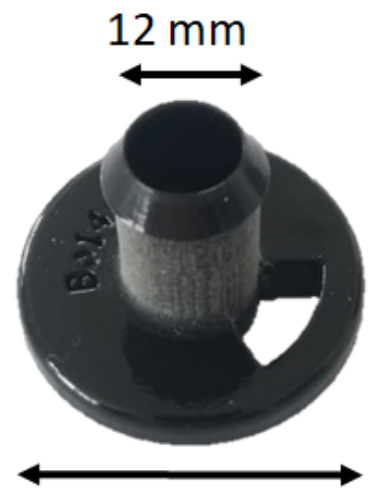

$31 \mathrm{~mm}$ (c) Straight B. Impeller.

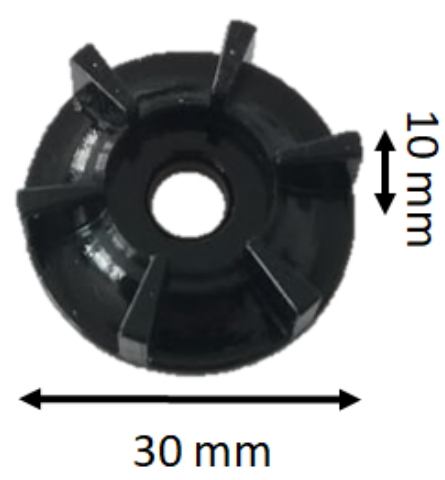

\subsection{Experimental Setup}

In order to test the built prototype, an experiment is performed. The experiment aims to build the curve pressure head versus mass flow given by the pump. Thus, the experiment consists of a fluid reservoir, a flow sensor (Flownetix 100series Smart Ultrasonic Flowmeter) and pressure sensors coupled to the inlet and outlet of the pump. The impeller rotation speed is manually controlled by changing the DC source output and observing the laser tachometer. The lid has a small window covered with a PET (Polyethylene terephthalate) plastic layer that allows the laser to reach a marker in the impeller. The experimental setup has a limitation in the rotational speed range of operation. It can operate the pump only at rotations between 1500 and $4000 \mathrm{rpm}$. The scheme representing this assembly is shown in Figure 9.4 . Figure 9.5 shows a picture of the experimental site.

Figure 9.4 - Experimental site scheme.

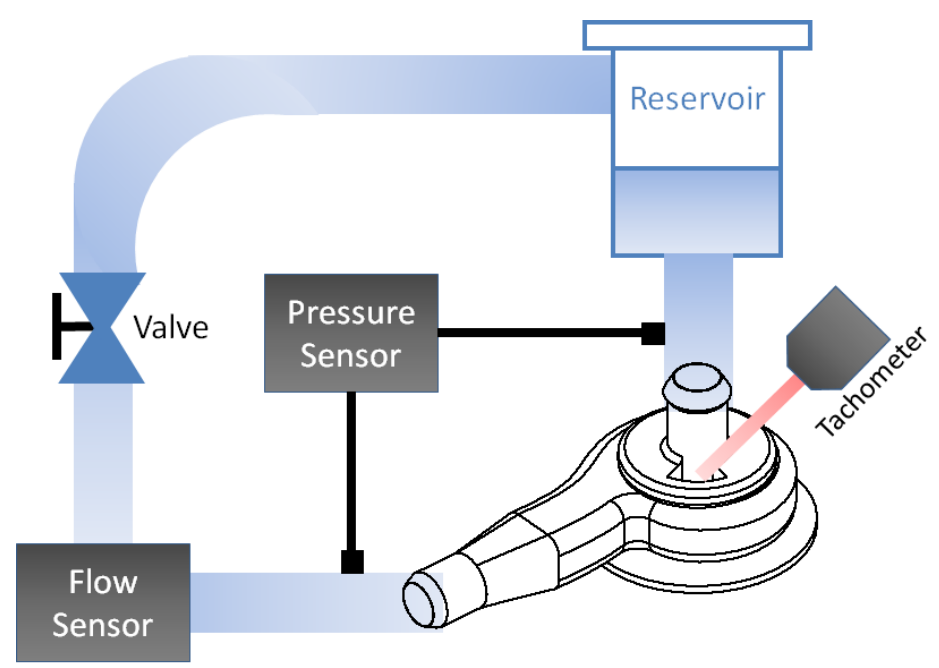


Figure 9.5 - Experimental site photo.

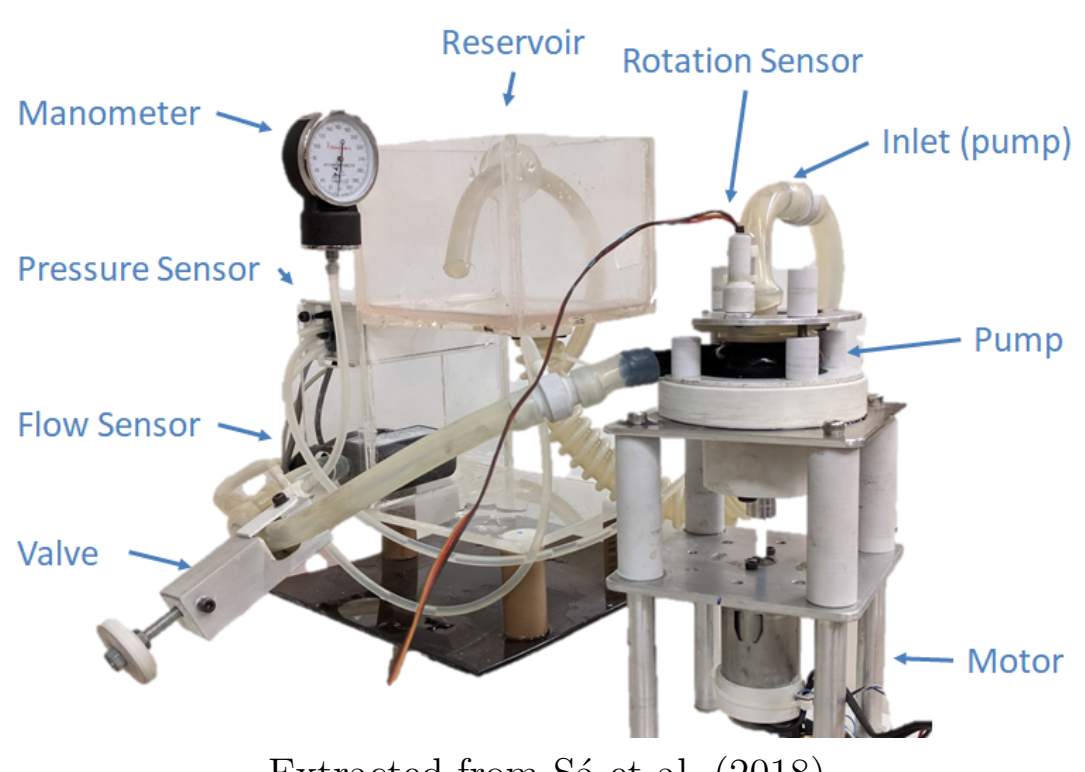

Extracted from Sá et al. (2018)

\subsection{Experiment Description}

The experiment consists of selecting a prototype (volute plus rotor), choosing an operation point, and then adjusting the rotation and valve opening that gives the desired pressure and mass flow. With the chosen rotation, the valve is gradually closed to collect the pump curve (pressure x mass flow and efficiency x mass flow). The prototype is changed, and the curve is collected for the chosen rotation. 


\section{EXPERIMENTAL RESULTS}

This chapter presents the experimental results for the impellers (Sec. 8.3) and volutes (Sec. 8.4.2) designs obtained via topology optimization of non-Newtonian turbulent flow. However, the experiments are performed with water. Results considering the same methodology applied to Newtonian laminar flow are published in the work Sá et al. (2018).

This chapter also presents the verification of a complete 3D VAD model computed with water. After the model is verified, it is adjusted to consider blood. Two operation points are simulated. First, the optimized and the straight-bladed VAD are simulated at $3[\mathrm{~L} / \mathrm{min}]$ and 3,000 [rpm]. Then, the boundary conditions are adjusted to consider the operation of an adult VAD (mass flow of $5[\mathrm{~L} / \mathrm{min}]$ and pressure of $100[\mathrm{~mm} \mathrm{Hg}]$ ), and the results are analyzed.

\subsection{Optimized non-Newtonian Turbulent Impeller}

This section presents the experimental results of the optimized impeller design, obtained with the non-Newtonian fluid and the turbulence models (Sec.8.3). The optimized topology is post-processed by using the software Autodesk Inventor (CAD) to create the 3D design with the necessary changes to accommodate the magnet and aluminum discs. The process from the optimized topology to the prototype is exemplified in Fig. 10.1. The volute used in the experiments has the optimized topology (Fig. 8.42).

Figure 10.1 - Impeller prototype creation process.

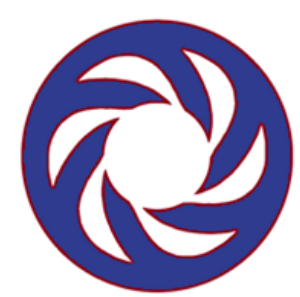

Optimized Topology

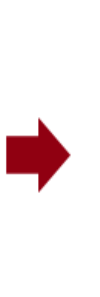

Post-processed CAD

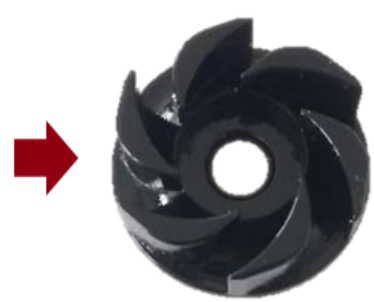

Prototype 


\subsubsection{Experimental Data}

The experimental results are the curves of pressure by mass flow and can be seen in Fig. 10.2. These curves are collected by measuring the differential pressure between the pump inlet and outlet and by measuring the mass flow. The horizontal error bar represents the uncertainty on measurement, which is assumed to be $0.05 \mathrm{~V}$ (or $0.16 \mathrm{~L} / \mathrm{min}$ ) for the flow sensor and $0.1 \mathrm{~V}$ (or $4.95 \mathrm{~mm} \mathrm{Hg}$ ) for the pressure sensor. These values are derived from both the systematic (deviations of the sensors) and random errors (fluctuation of the measure).

The fluid used is water, given that, from past experiments performed at our laboratory, the blood analog fluid (WALKER; JOHNSTON; RIVAL, 2014) behaves very similarly to water at our conditions, i.e., the pressure by mass flow curves were very similar. This result is also reported in the literature (TANG et al., 2004), where it is reported that the Newtonian assumptions in the macroscopic modeling are of the lowest order of importance, affecting measured output by less than $10 \%$.

Comparing the pressure outputs (Fig. 10.2), it is possible to observe that the straight blade rotor presents a higher pressure head than the optimized impeller. This phenomenon is standard in the literature, and the straight-bladed rotors tend to present higher pressure than the traditional backward-facing blades (which are very similar to the optimized blades). Around $2 \mathrm{~L} / \mathrm{min}$ the straight-bladed impeller shows an abrupt pressure reduction, which may be caused by a misalignment of the center of rotation. The magnetic bearing allows for some movement in the radial direction, and the difference of pressure at the volute displaces the impeller.

Figure 10.2 - Experimental curve for straight and optimized blade prototypes.

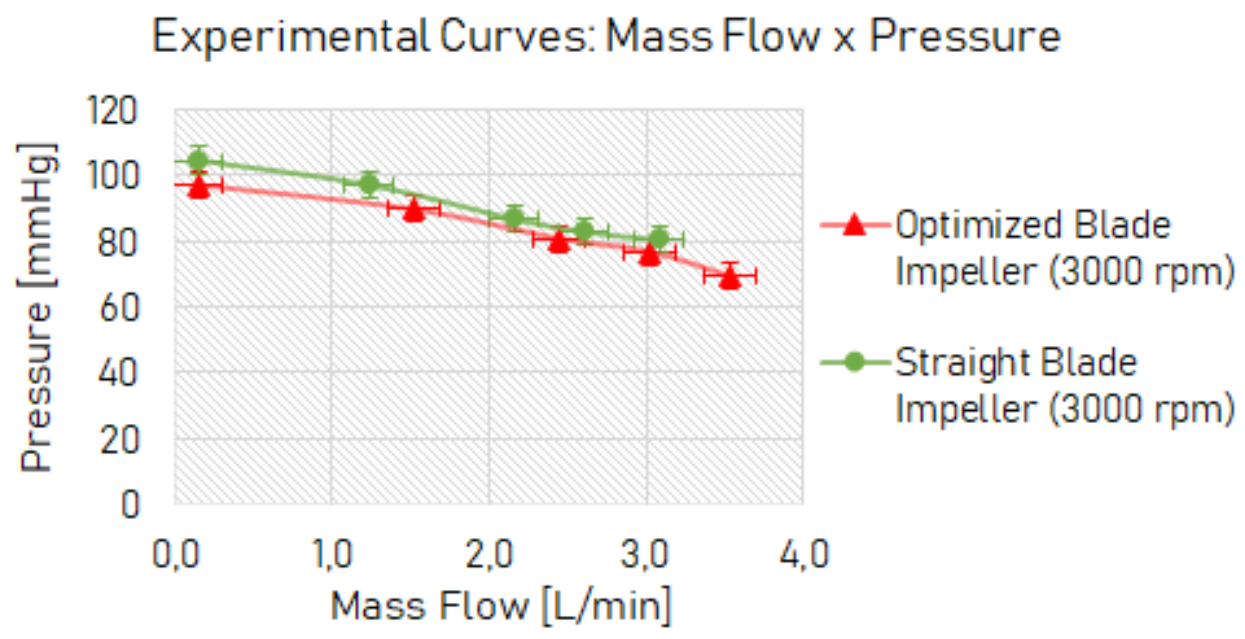


The current experimental data do not provide much information about the flow inside the pump, neither about the shear rate. Thus, the 3D simulation of the complete pump (impeller and volute) is performed in the following sections. First the experimental data is used to calibrate the ANSYS simulation model with water as fluid, by adjusting the mesh, the boundary conditions, the turbulence model and the solver. Secondly, the fluid is changed to blood and the model is verified at the design operation point of $3[\mathrm{~L} / \mathrm{min}]$ and $3000[\mathrm{rpm}]$. Finally, the model is adjusted to simulate the operation of the VAD as and adult VAD operating at $5[\mathrm{~L} / \mathrm{min}]$ and $100[\mathrm{mmHg}]$. These steps are shown in Fig. 10.3 .

Figure 10.3 - Simulation Analysis Steps.

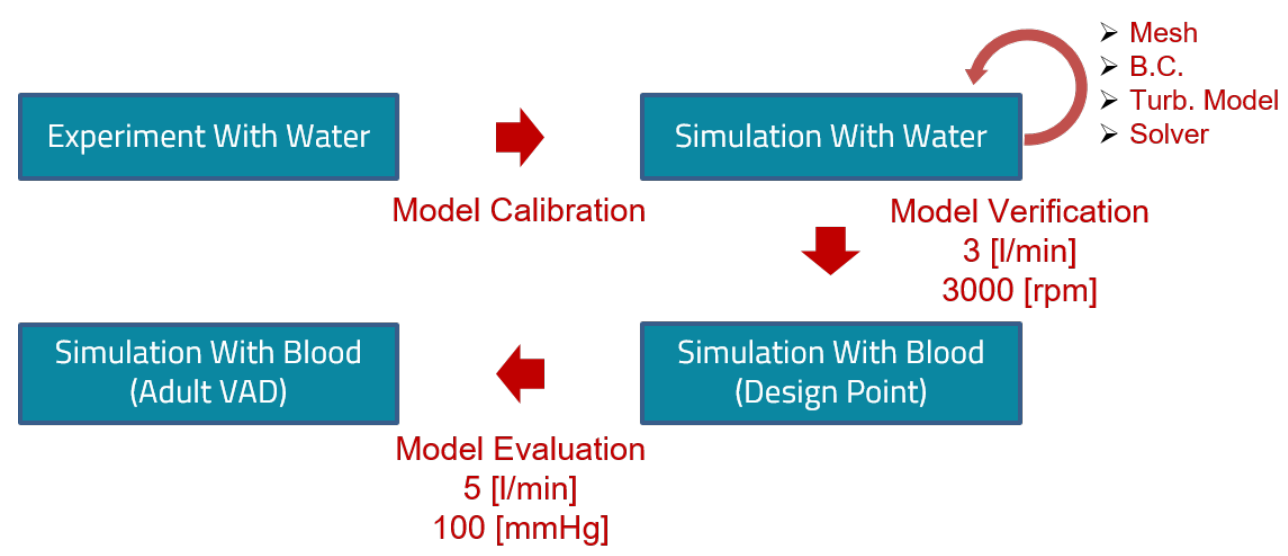

\subsubsection{Computational Comparison (Model Calibration)}

The experimental data can be used to define the boundary conditions of the computational model, by using the prototype mass flow information to define the model inlet velocity and to set the rotational speed of the impeller. The verification of the model is done by comparing the model pressure gain with the experimentally measured pressure gain.

The simulation is done considering the complete 3D pump model with six blades coupled with the volute casing by using the commercial software ANSYS. This simulation allows the observation of the velocity and pressure distributions over the complete pump model. The $k-\omega S S T$ turbulence model is used. Water is used as the simulation fluid. The boundary conditions for the 3D model is shown in Fig. 10.4. The impeller model (Fig. 10.4a) has a rotating wall condition on the surface of the blades and discs, and a Frozen Rotor interface condition on the frontier of the fluid domain. The volute model (Fig. 10.4b has a Mass Flow Inlet condition at the inlet face, a Stationary Wall condition at the walls, and zero Static Pressure condition at the outlet faces. The mesh convergence analysis is shown in Appendix D. 
Figure 10.4 - Boundary Conditions for the ANSYS model (complete pump).

(a) Impeller B.C.

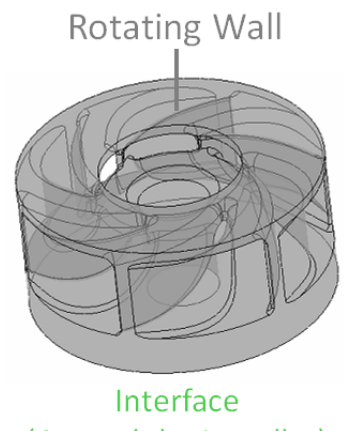

(Around the Impeller) (b) Volute B.C.

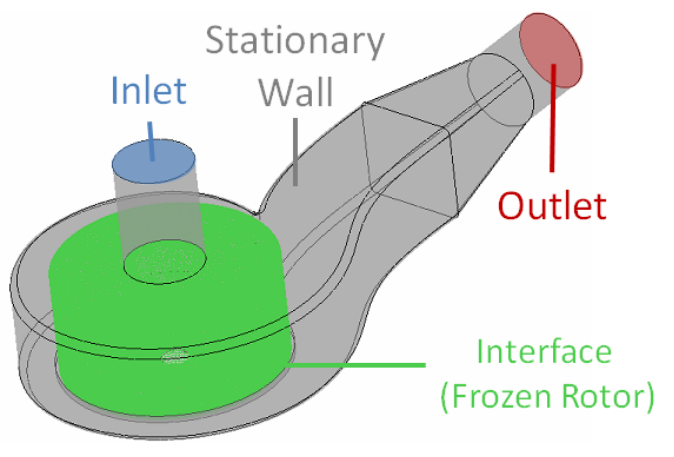

First, a verification of the $A N S Y S$ model is done by comparing the experimental data with the results from the model. The mass flow by pressure curves for the optimized impeller are shown in Fig. 10.5a, Given that each simulation takes about one day and 11 hours to converge only a few points are simulated. The curves show good agreement for the mass flow higher than $1.5[L / m i n]$. The points with low mass flow values (and high rotations) present large recirculation zones and may become time-dependent, affecting the turbulence model, which may not calculate the eddy formation correctly under the current settings (mesh size and steady-state assumption). However, this does not affect much the current work, because the region of interest is around $3[\mathrm{~L} / \mathrm{min}]$. Figure $10.5 \mathrm{~b}$ shows the verification for the straight blade impeller. The same detachment is observed for the low mass flow values. Besides, at the region around $3[\mathrm{~L} / \mathrm{min}]$ the error between the curves is more pronounceable, giving an error around $8 \%$. The error value increase occurs due to the strong recirculation present in the straight blade, which causes an increase of the pressure in the simulation. However, this error percentage is still around the expected value when considering turbulence models, which can give modeling errors up to $20 \%$. 
Figure 10.5 - Experimental and Computational Data Comparison.

(a)

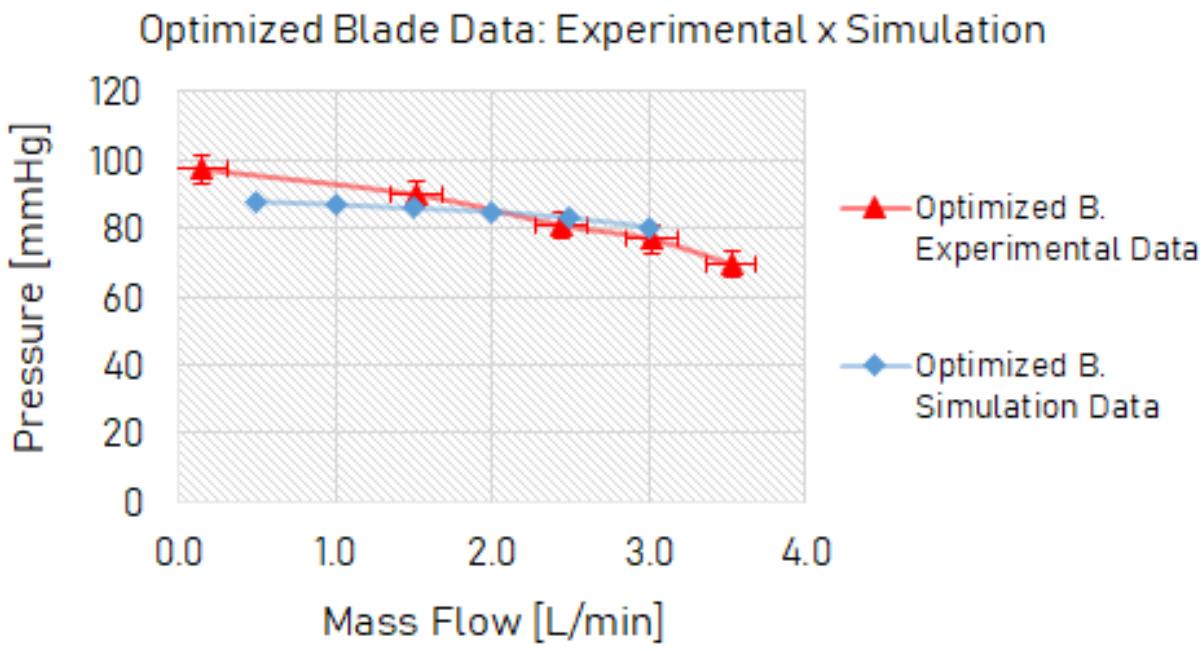

(b)

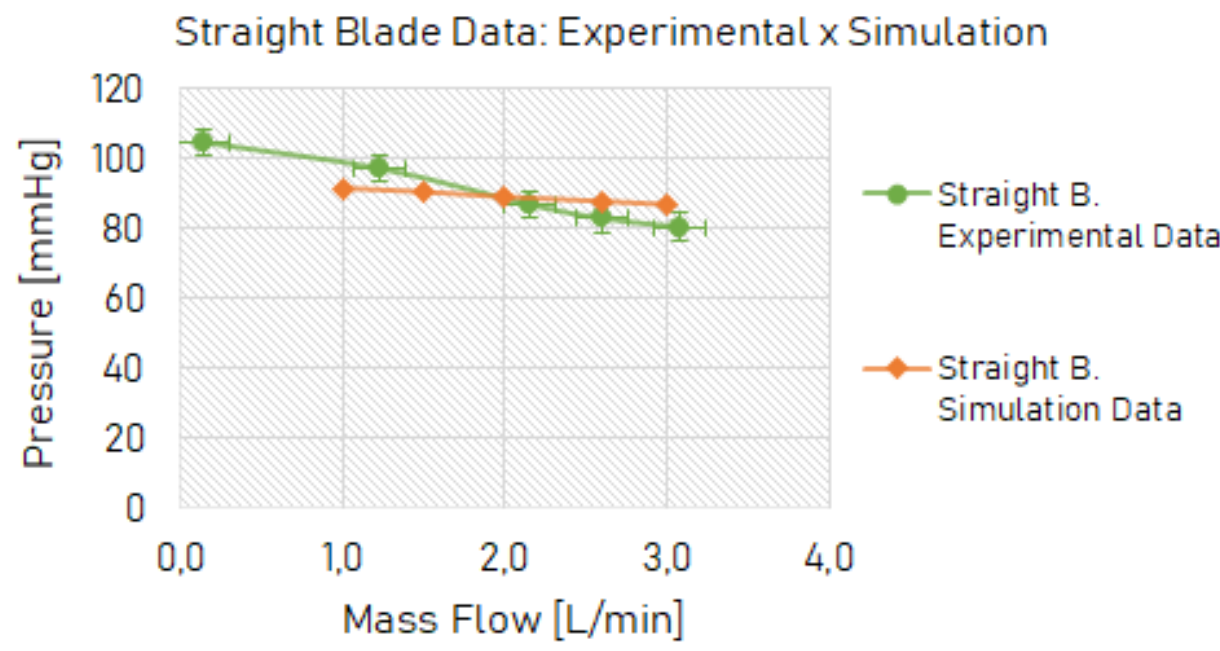

The verification shows a better agreement of the experimental and simulated curves for the optimized blades. However, it does not invalidate the functional comparison for the regions where they are coherent. Hence, Figure 10.6 shows the comparison of the energy dissipation and vorticity functionals (integrated over the entire domain) for different mass flows. It can be seen that both the energy dissipation and vorticity curves have the same shape, confirming once more that both functionals have a high correlation. In addition, the optimized blade has an almost constant energy dissipation (Fig. 10.6a) with a sudden decrease near $3[\mathrm{~L} / \mathrm{min}]$, which is the design point (for both impeller and volute). This suggests that, for each operational point, a local minimum should exist. The same effect is observed for the straight blade impeller, as the mass flow increase the flow condition becomes closer to the volute design point, and the energy dissipation value decreases. Finally, the optimized impeller performs better than the straight-bladed impeller for all 
mass flows analyzed. The optimized impeller has both the energy dissipation and vorticity values around $40 \%$ lower than the straight blade case.

Figure 10.6 - Energy Dissipation and Vorticity Comparison between Straight and Optimized Blade Impellers.

(a)

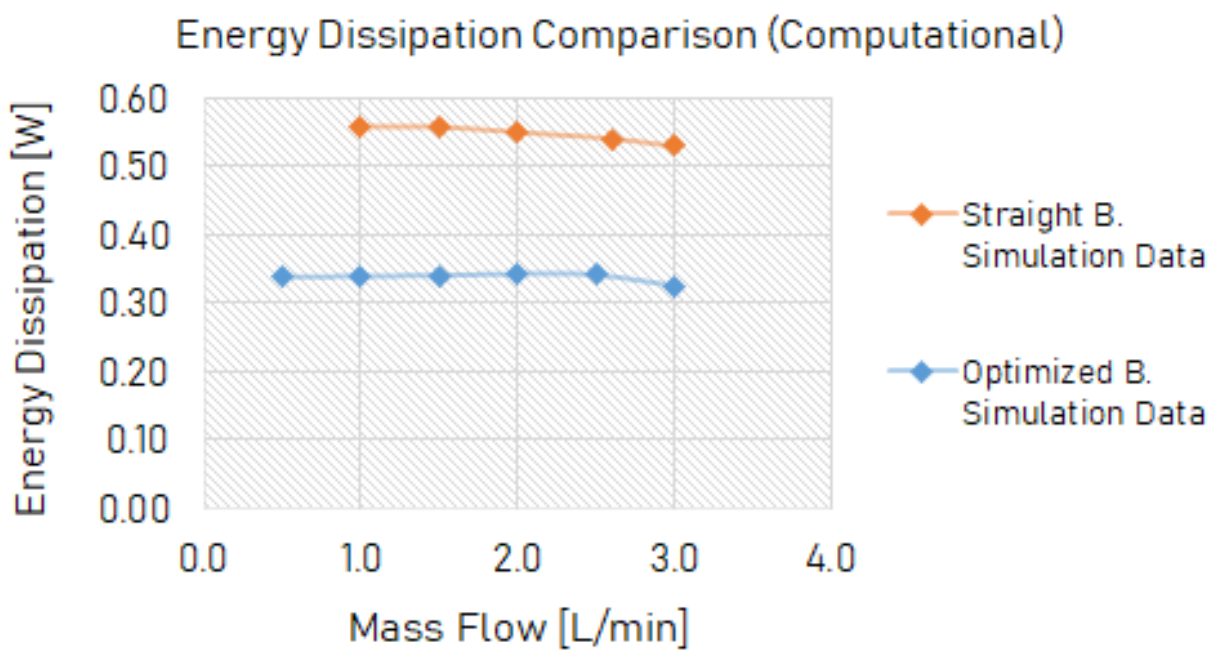

(b)

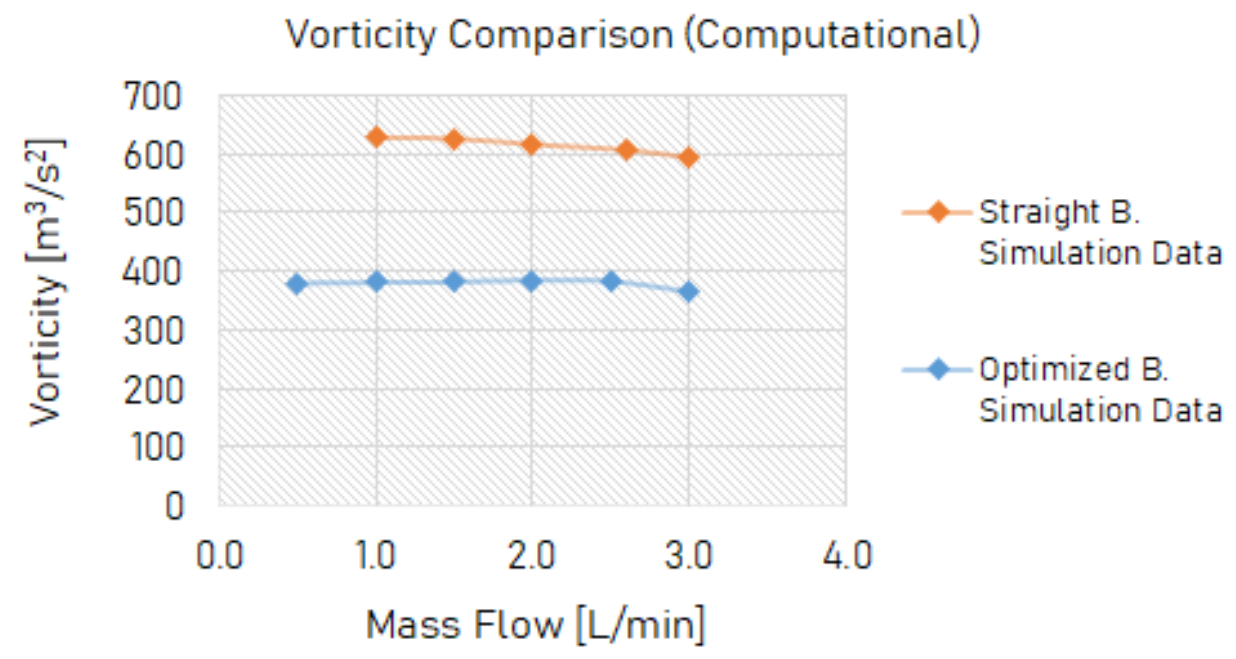

The $3 \mathrm{~L} / \mathrm{min}$ point is further analyzed by collecting the velocity, pressure, energy dissipation, vorticity, and turbulent viscosity plots for both impellers. Figures 10.7 and 10.8 shows the field plots for the optimized and straight bladed impellers, respectively. These plots are collected on the impeller surface and a plane passing at half height.

The velocity fields (Figs. 10.7a and 10.8a) show that the peak velocity is higher in the straight bladed impeller (SB). The velocity pattern is more homogeneous among the blades of the optimized case, while the SB case presents some blades with peak velocity of $6.3[\mathrm{~m} / \mathrm{s}]$ and others with $2.3[\mathrm{~m} / \mathrm{s}]$. The same behavior is observed for the pressure 
fields (Figs. 10.7b and 10.8b), being the minimal pressure lower in the SB than in the optimized case, i.e., the pressure gain is higher in the SB case. The SB eddy viscosity field (Fig. 10.8c) shows that the blade passing by the cutwater presents a high eddy production, while, in the optimized case (Fig. 10.7c), the amplitude of values is lower.

Figure 10.7 - Variable Fields for the optimized impeller ANSYS simulation at $3[L / \mathrm{min}]$ and $3000[$ rpm $]$.

(a) Velocity Field.

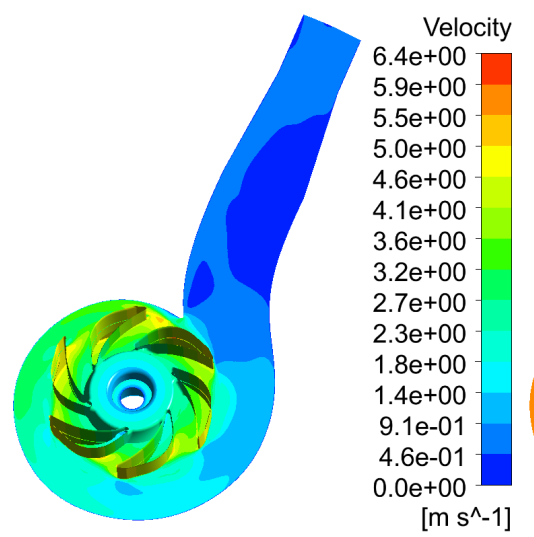

(d) Shear Strain Rate.

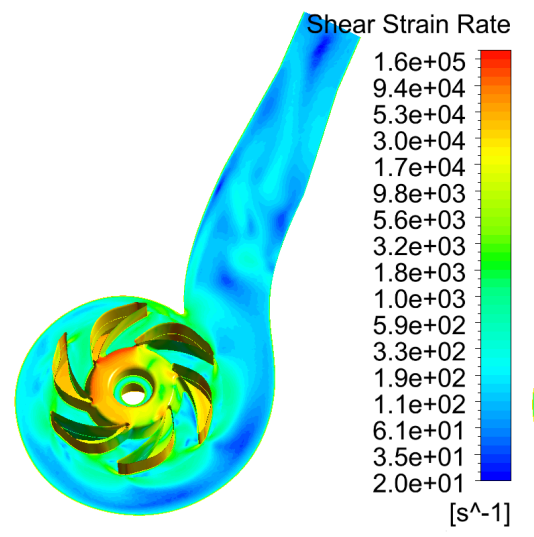

(b) Pressure Field.

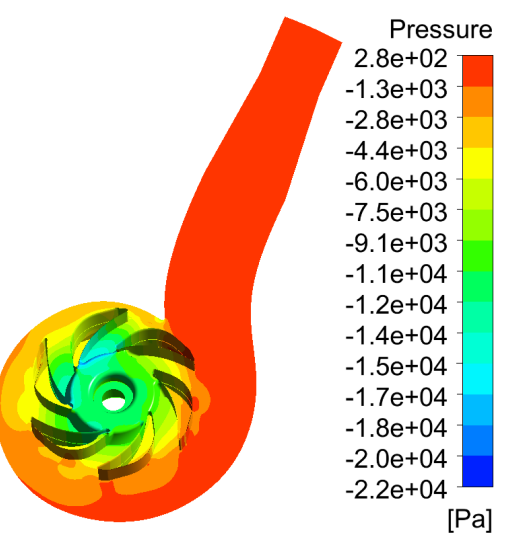

(e) Energy Dissipation.

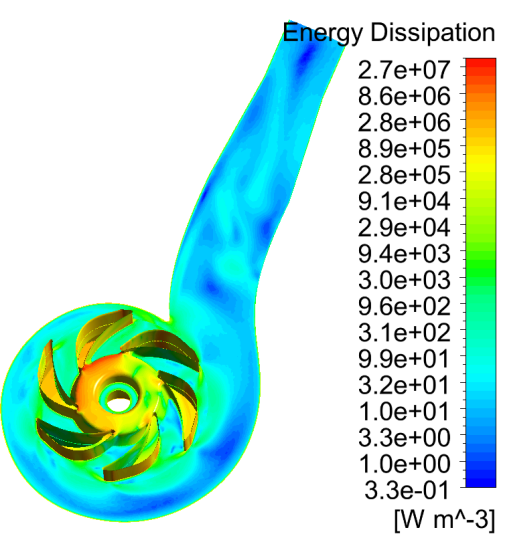

(c) Eddy Viscosity Field.

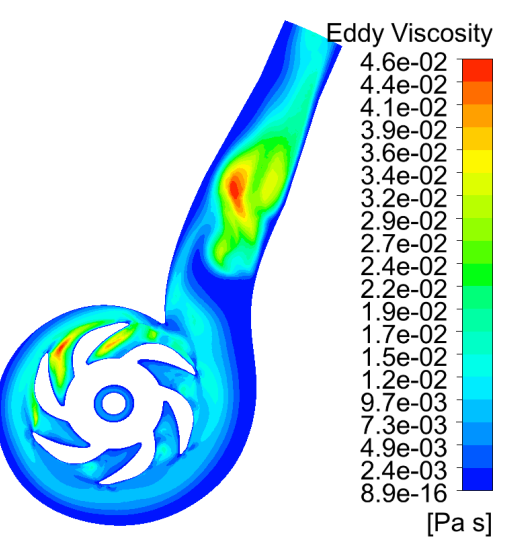

(f) Vorticity.

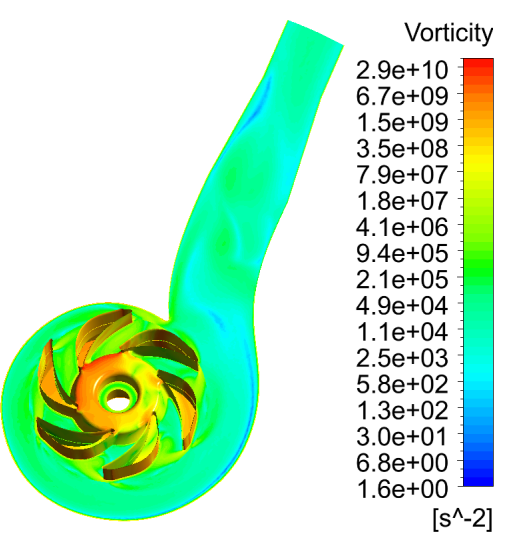

The shear strain rate distribution is the scalar shear stress plotted in a point-wise manner. This distribution is smoother in the optimization case (Fig. 10.7d), with small variations along the volute path. The distribution in the SB case (Fig. 10.8d) reveals the opposite, there are regions with high amplitudes and abrupt changes. The peak is lower in the optimized case than in the SB case, and it occurs in small portions of the pump. This reflects in the lower integrated value, as seen in Fig. 10.6a.

Looking at the energy dissipation distribution (Figs. 10.7e and 10.8e and comparing it with the shear strain rate distribution (Figs. $10.7 \mathrm{~d}$ and $10.8 \mathrm{~d}$ ) we can see that they have the same pattern, and the differences are only at the scale values. Thus, reinforcing the assumption (from Sec. 5.3.1) that they have the same behavior and minimizing one implies in the minimization of the other. This could also be inferred to the vorticity by comparing the plots on the impeller surface (Figs. 10.7f and 10.8f) with the energy dissipation plots, 
which are not exactly proportional but have the same pattern.

Figure 10.8 - Variable Fields for the straight bladed impeller (SB) ANSYS simulation at 3 $[L / \mathrm{min}]$ and $3000[\mathrm{rpm}]$.

(a) Velocity Field.

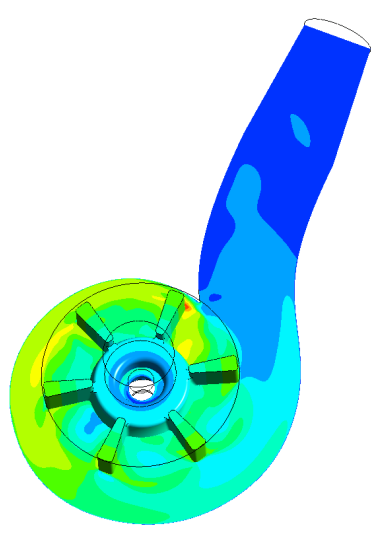

(d) Shear Strain Rate.

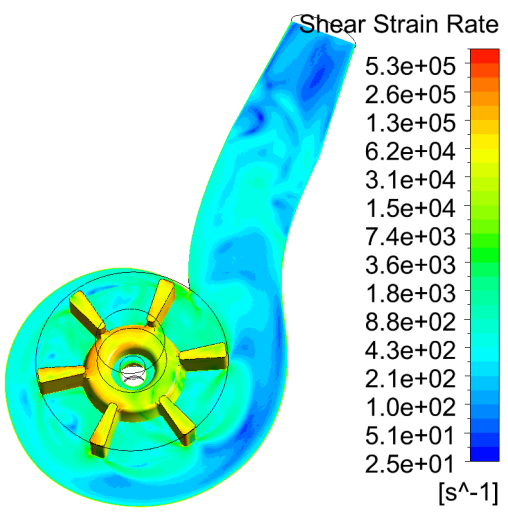

(b) Pressure Field.
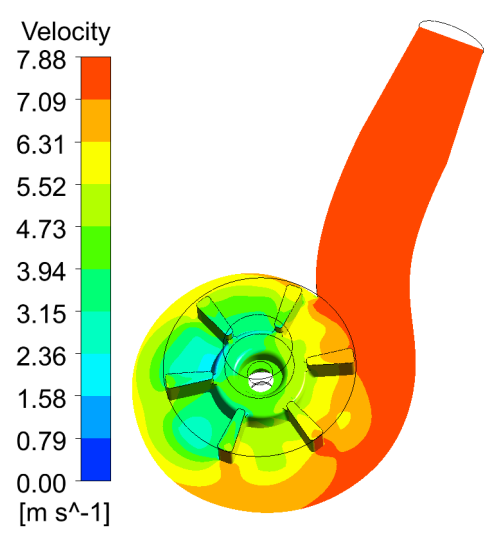

(e) Energy Dissipation.
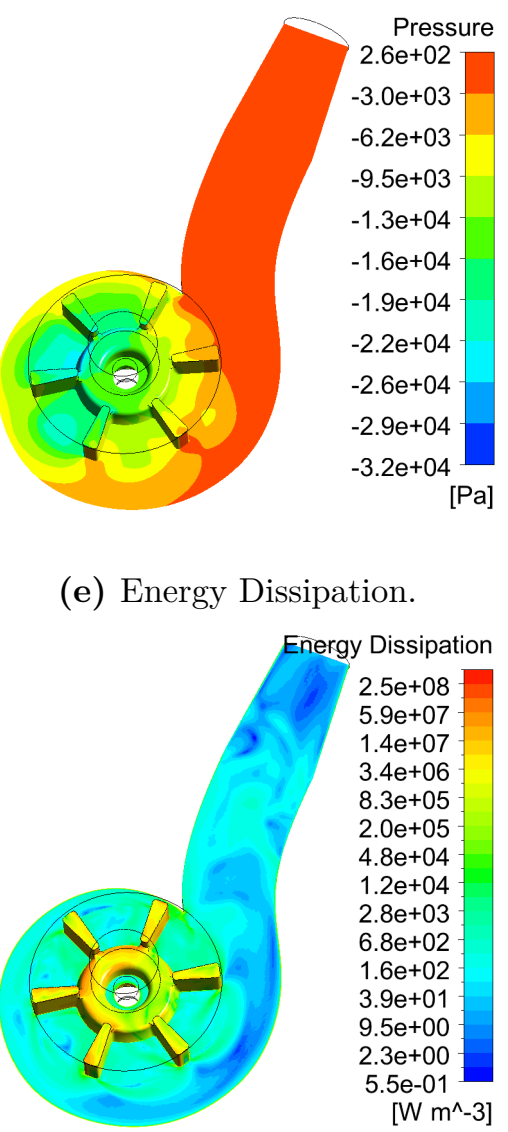

(c) Eddy Viscosity Field.

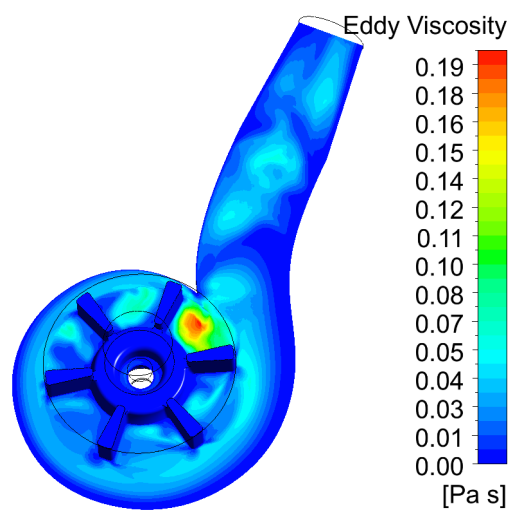

(f) Vorticity.

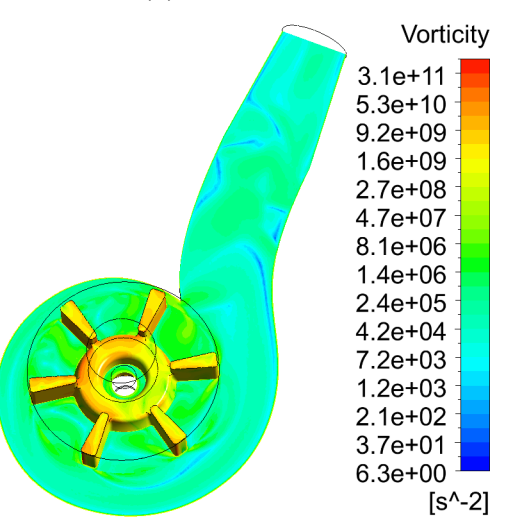

The vorticity plots (Figs. $10.7 f$ and $10.8 f$ ) show that most of the vorticity is generated on the impeller. The optimized case distribution is more homogeneous and presents very few points with maximum value. The SB case vorticity distribution has a higher maximum value and presents more zones with high values (higher than $1.4 \cdot 10^{6}$ ) which causes it to have a higher functional value when integrated over the domain 10.6b.

Hence, the variable distributions are more homogeneous for the optimized impeller, and the functional values (integrated over the domain) are lower than in the straight-bladed impeller. Indicating that the optimization process performed well, resulting in a better impeller for the given conditions.

\subsubsection{Simulation with Blood $(3[\mathrm{~L} / \mathrm{min}]$ and $3000[\mathrm{rpm}])$}

The computational models are verified for the experiments with water. Even though no experiment is performed with blood, the same computational model can be used to simulate the operation with blood. Hence, the same boundary conditions, shown in Fig. 
10.4 in combination with the Carreau-Yasuda non-Newtonian model (blood), are used to simulate the operation of both impellers, optimized and straight-bladed (SB), at the operation point used in the optimization $(3[\mathrm{~L} / \mathrm{min}]$ and $3000[\mathrm{rpm}])$.

Figure 10.9 shows the variable fields for the optimized impeller simulation, while Fig. 10.10 shows the fields for the straight-bladed impeller. These plots are collected at a plane at half height and on the impeller surface.

Comparing the velocity plots (Figs. 10.9a and 10.10a) it is possible to notice that the optimized impeller has a more homogeneous velocity distribution among its blades, while the SB case has a concentration of high velocities at the blade closer to the cutwater. The pressure plots (Figs. $10.9 \mathrm{~b}$ and 10.10b) show that in the SB the minimum value is lower than in the optimized case, which is expected, given that the experiments have shown that the SB generates a higher pressure jump.

The dynamic viscosity plots display the non-Newtonian viscosity field on the half-height plane. The impeller surface plot is removed because it presents the lowest viscosity values due to the high velocity gradients. The viscosity distribution of the optimized case (Fig. $10.9 \mathrm{c}$ shows some intermediary values between the blades (around $4 \cdot 10^{-3}[\mathrm{~Pa} \cdot \mathrm{s}]$ ) while in the SB plot (Fig. 10.10c) the lowest scale value is predominant, this indicates higher shear rates in the SB case.

The eddy viscosity plots show that the eddy formation occurs in different regions for each impeller. The high eddy viscosity occurs at the left portion of the optimized case (Fig. 10.9d), while it occurs in the left part of the SB plot (Fig. 10.10d). Also, the peak value for the optimized case is almost half of the SB case. Thus, the SB impeller introduces more turbulence in the flow than the optimized case.

The energy dissipation (Figs. 10.9e and 10.10e and vorticity plots (Figs. 10.9f and 10.10f exposes the same behavior described in the Section 10.1.2, in which the optimized case shows lower peak values. However, the operation with blood gives rise to the hemolysis condition. Therefore, the wall shear stress is analysed.

By analysing the wall shear stress in the impellers (Figs. $10.9 \mathrm{~g}$ and $10.10 \mathrm{~g}$ ) it is possible to notice that the peak values occur at the blade inlet. The optimized impeller demonstrates a lower peak value, and most of the distribution is between 0 and $140[P a]$ while the SB impeller is between 0 and $170[\mathrm{~Pa}]$. Similar behavior is observed in the volute wall shear stress plots, and the peak values are very close. However, the overall distribution presents lower values in the optimized case (Fig. 10.9h), i.e., there are more regions with the light blue color (values up to $150[P a]$ ). While the SB case overall distribution (Fig. $10.10 \mathrm{~h}$ reaches the green coloration (values up to $230[\mathrm{~Pa}]$ ). Also, it is important to notice that the peak value for both volute plots occurs at the cutwater. 
Figure 10.9 - Variable Fields for the optimized impeller ANSYS simulation with blood at 3 $[L / \mathrm{min}]$ and $3000[\mathrm{rpm}]$.

(a) Velocity Field.

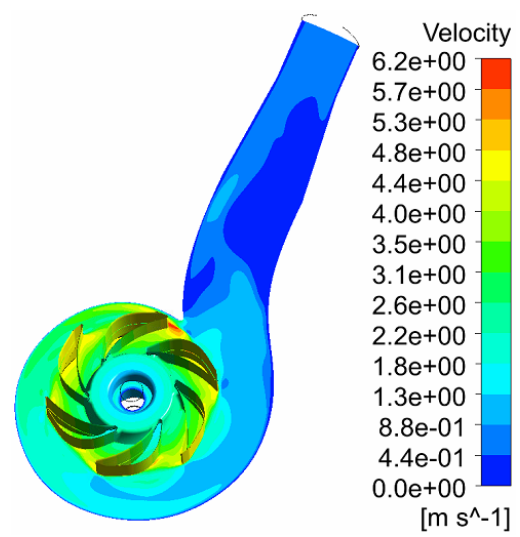

(d) Eddy Viscosity.

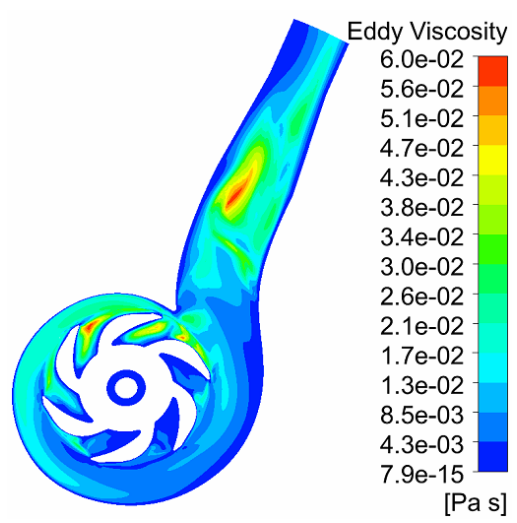

(g) Shear Stress (Impeller). (b) Pressure Field.

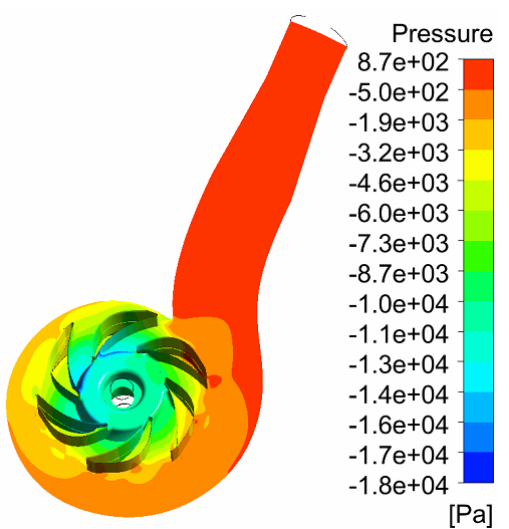

(e) Energy Dissipation.

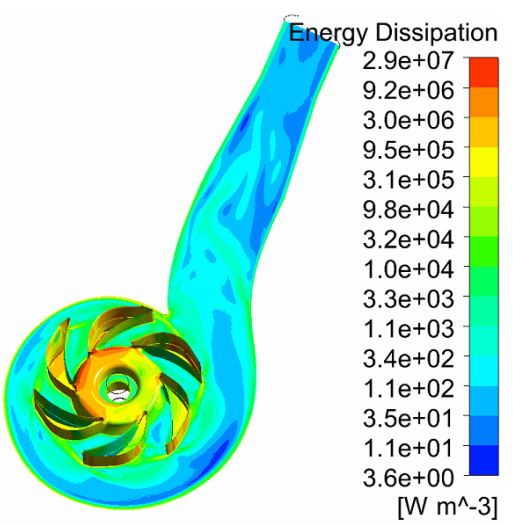

(c) Non-Newtonian Viscosity.

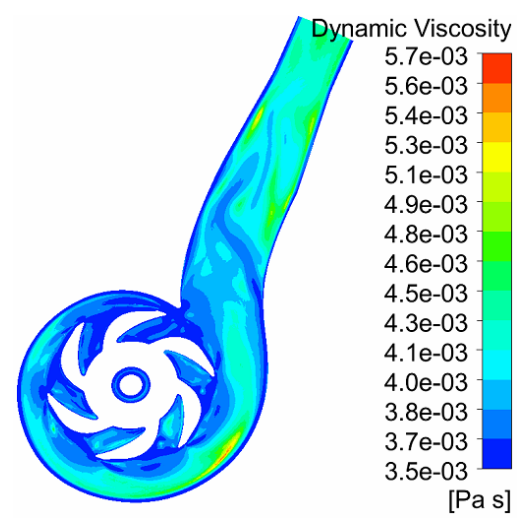

(f) Vorticity.

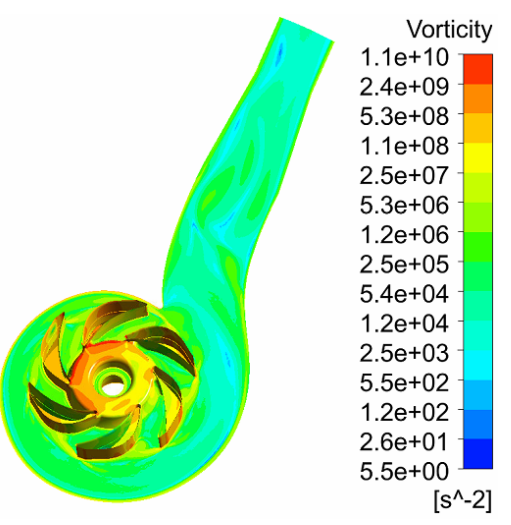

(h) Shear Stress (Volute).

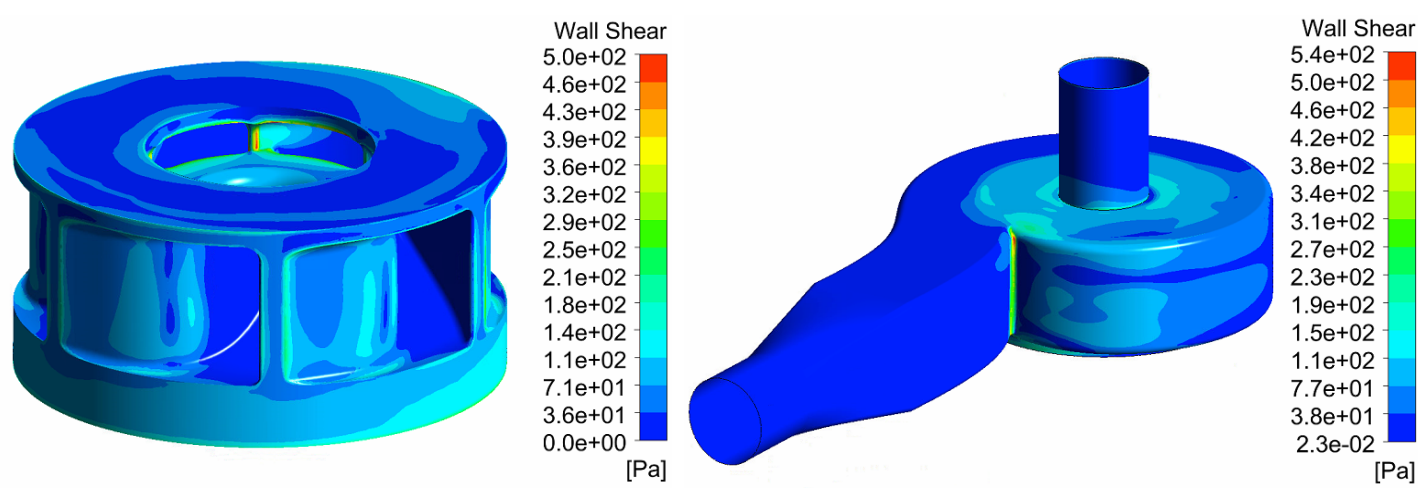


Figure 10.10 - Variable Fields for the straight-bladed impeller ANSYS simulation with blood at $3[\mathrm{~L} / \mathrm{min}]$ and $3000[\mathrm{rpm}]$.

(a) Velocity Field.

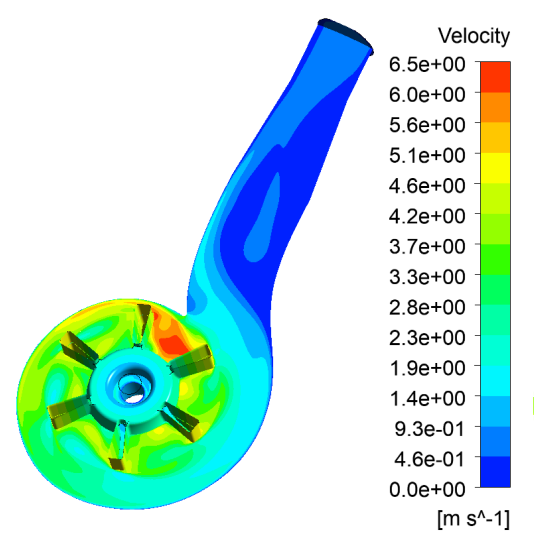

(d) Eddy Viscosity.

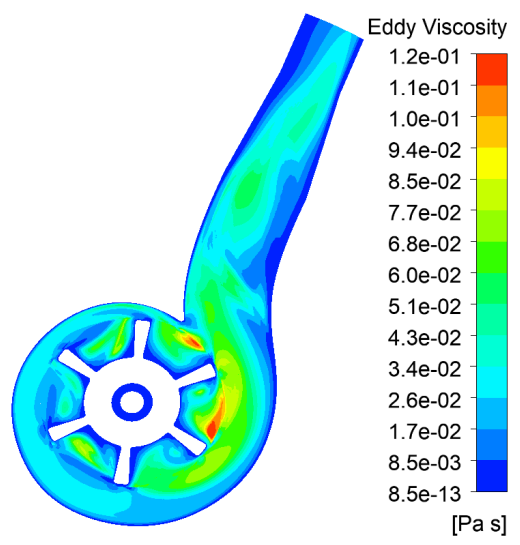

(b) Pressure Field.

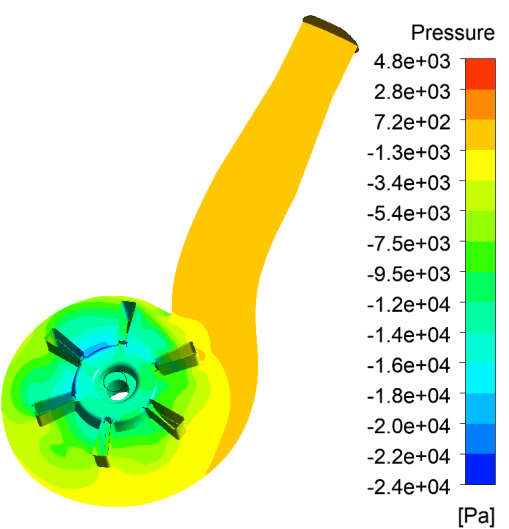

(e) Energy Dissipation.

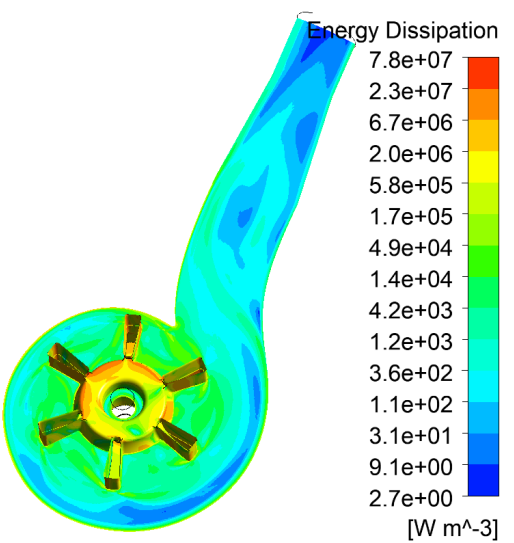

(c) Non-Newtonian Viscosity.

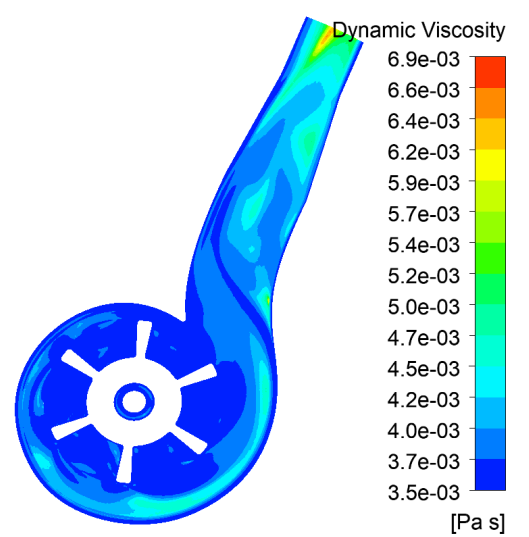

(f) Vorticity.

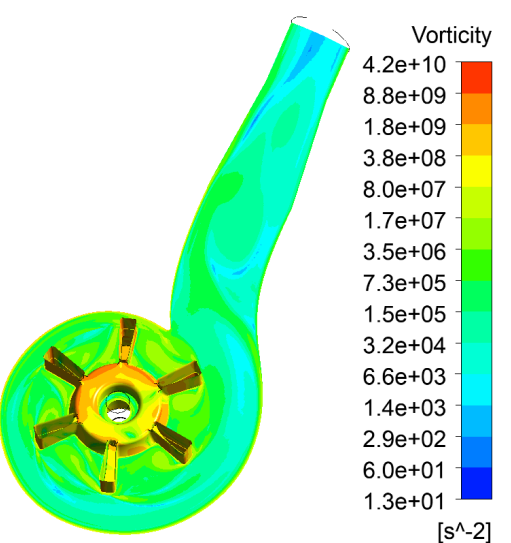

(g) Shear Stress (Impeller).

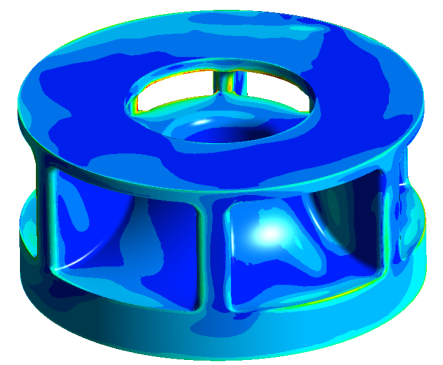

(h) Shear Stress (Volute).

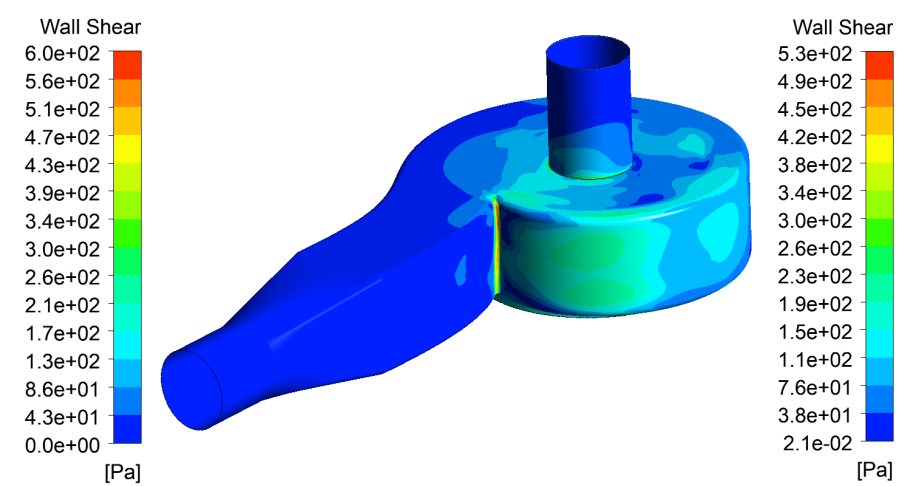


The functional values for both cases are shown in Tab. 10.1, Due to the difference in the wall shear stress observed for the impeller and volute plots, the table values are split by domain, i.e., the impeller values are computed by integrating the functional in the impeller volume, and the volute values in the volute volume. The total values are the sum of the impeller and volute functionals.

In Tab. 10.1, the optimized case provides an overall improvement around $32 \%$, for both the impeller and volute domains. The optimized curvature in the blades of the impeller improves the domain values. While the better values of the volute domain are associated with the velocity angles exiting the impeller being closer to the angles used at the volute optimization. This indicates that it is crucial to have the correct combination between impeller and volute to achieve the best functional values.

Table 10.1 - Functional values Straight-bladed and Optimized impellers operating with blood at $3[\mathrm{~L} / \mathrm{min}]$ and $3000[\mathrm{rpm}]$.

\begin{tabular}{llccc}
\hline & & Straight Blade & Optimized & Difference (\%) \\
\hline Mass Flow & {$[\mathrm{L} / \mathrm{min}]$} & 3 & 3 & - \\
Rotation & {$[\mathrm{rpm}]$} & 3000 & 3000 & - \\
Pressure & {$[\mathrm{mmHg}]$} & 93 & 81 & $-12 \%$ \\
& & & & \\
Impeller & & & & \\
Energy Dissip. & {$[\mathrm{W}]$} & 0.41 & 0.28 & $-32 \%$ \\
Vorticity & {$\left[\mathrm{m}^{3} / \mathrm{s}^{2}\right]$} & 117 & 80 & $-32 \%$ \\
& & & & \\
Volute & & & & \\
Energy Dissip. & {$[\mathrm{W}]$} & 0.49 & 0.33 & $-32 \%$ \\
Vorticity & {$\left[\mathrm{m}^{3} / \mathrm{s}^{2}\right]$} & 137 & 93 & $-32 \%$ \\
Total & & & & \\
Energy Dissip. & {$[\mathrm{W}]$} & 0.90 & 0.61 & $-32 \%$ \\
Vorticity & {$\left[\mathrm{m}^{3} / \mathrm{s}^{2}\right]$} & 255 & 174 & $-32 \%$ \\
\hline
\end{tabular}

\subsubsection{Simulation with Blood (5 $[\mathrm{L} / \mathrm{min}]$ and $100[\mathrm{mmHg}])$}

In the previous section, the behavior of the VADs is analyzed by considering the mass flow $(3[\mathrm{~L} / \mathrm{min}])$ and rotation $(3000[\mathrm{rpm}])$ fixed between the cases. In this section, the application of these pumps as an adult VADs is analyzed by fixing the mass flow at 5 $[\mathrm{L} / \mathrm{min}]$ and adjusting the rotation until the pressure jump reaches $100[\mathrm{~mm} \mathrm{Hg}]$. Hence, the rotations are calibrated by using the similarity laws, so the optimized VAD simulation is run with $3400[\mathrm{rpm}]$ and the straight-bladed VAD simulation with $3200[\mathrm{rpm}]$.

Figure 10.11 displays the variable fields for the optimized impeller simulation, while 
Fig. 10.12 presents the fields for the straight-bladed impeller. These plots are collected at a virtual plane at half height and on the impeller surface. Due to the similarity between the plots presented here and the plots from the previous section, analogous conclusions can be drawn. The main finding is that higher peak values follow higher mass flows and rotation speeds.

Figure 10.11 - Variable Fields for the optimized impeller ANSYS simulation with blood at 5 $[L / \mathrm{min}]$ and $3400[\mathrm{rpm}]$.

(a) Velocity Field.

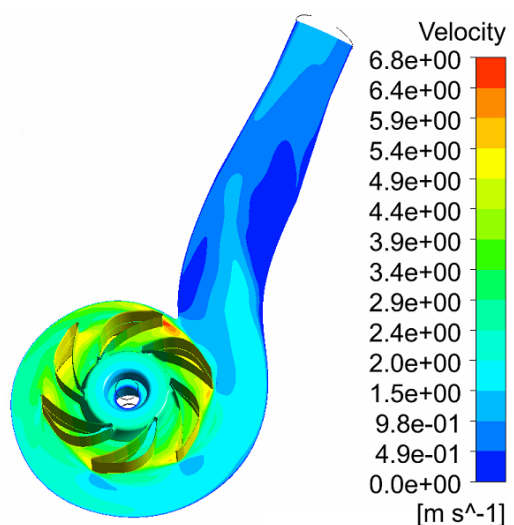

(d) Eddy Viscosity.

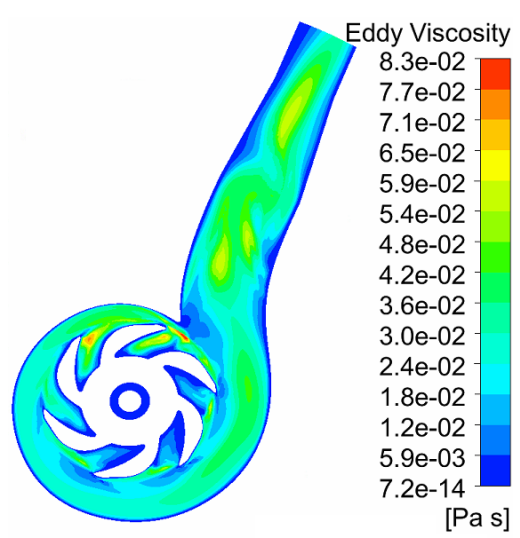

(e) Energy Dissipation.

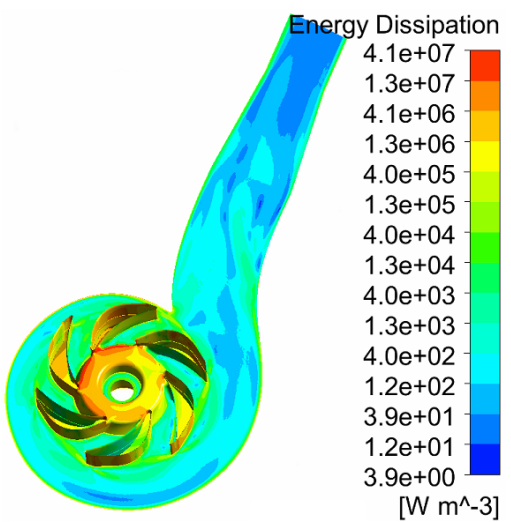

(c) Non-Newtonian Viscosity.

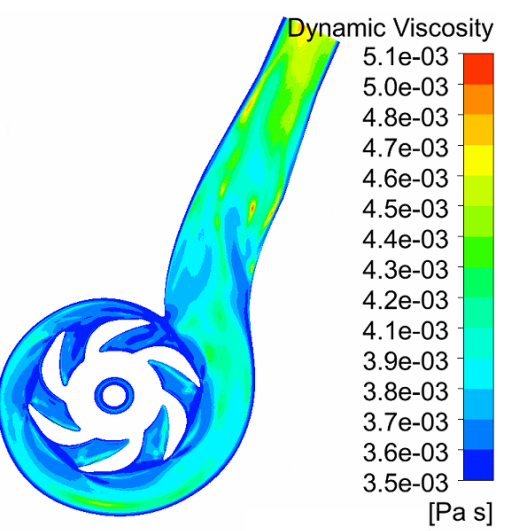

(f) Vorticity.

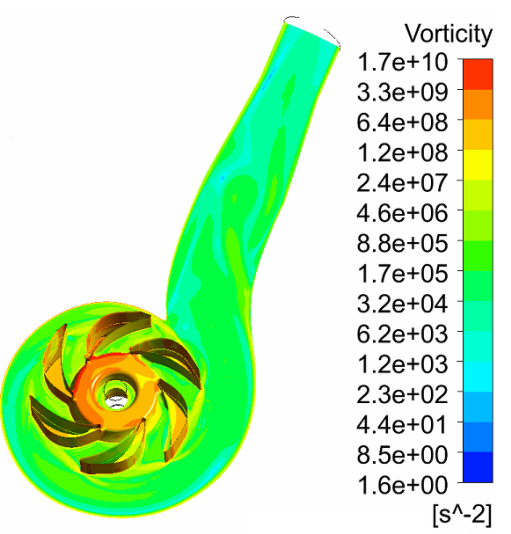

(g) Shear Stress (Impeller).

(h) Shear Stress (Volute).

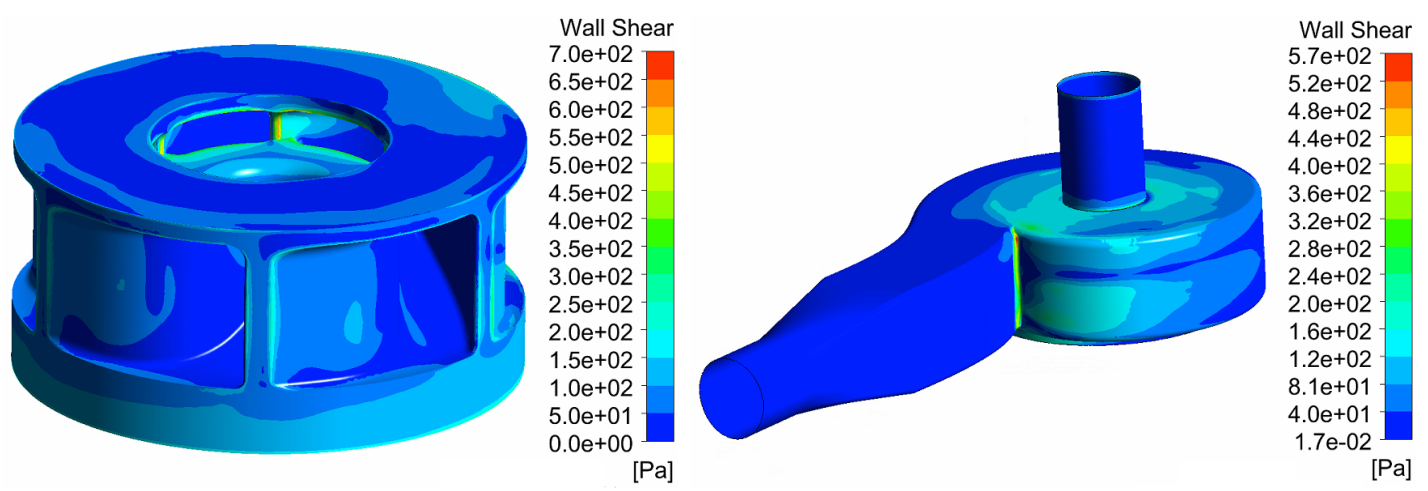


Figure 10.12 - Variable Fields for the straight-bladed impeller ANSYS simulation with blood at $5[L / \mathrm{min}]$ and $3200[\mathrm{rpm}]$.

(a) Velocity Field.

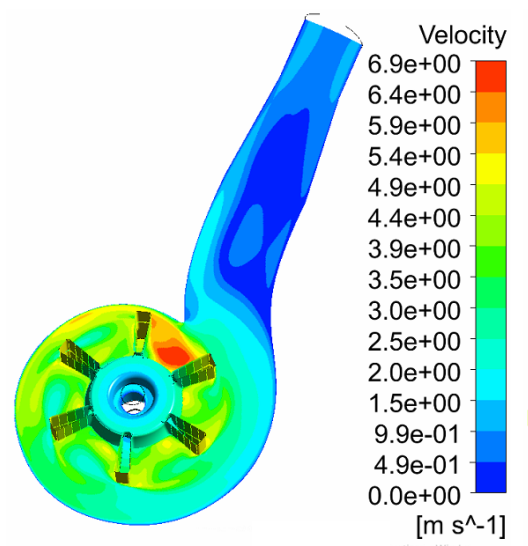

(d) Eddy Viscosity.

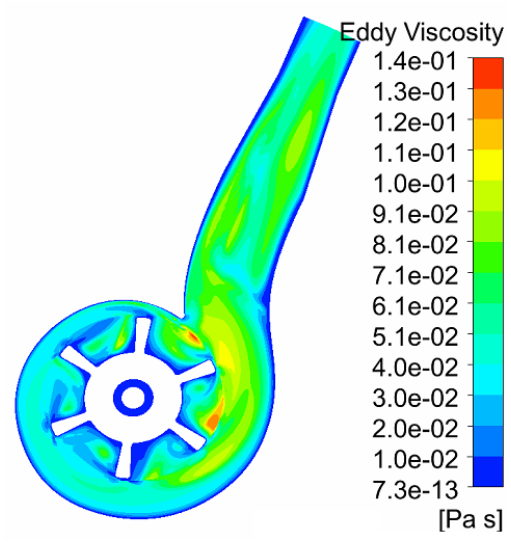

(g) Shear Stress (Impeller).

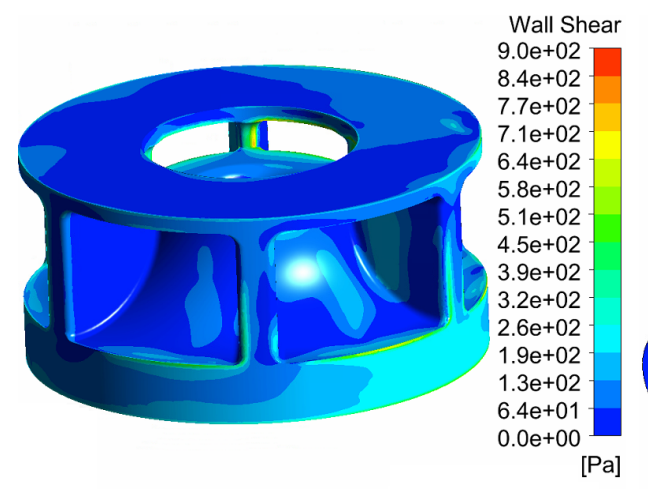

(b) Pressure Field.

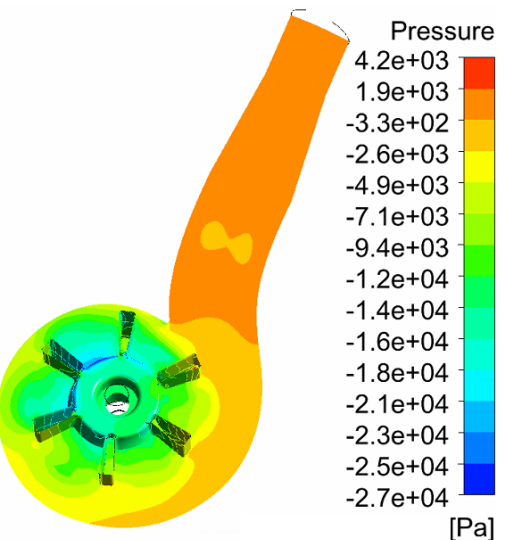

(e) Energy Dissipation.

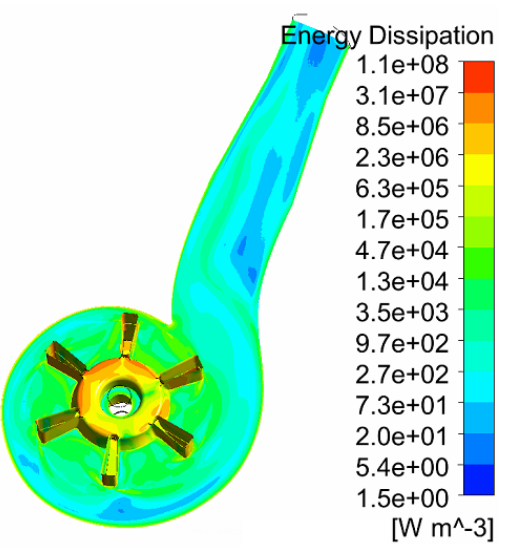

(c) Non-Newtonian Viscosity.

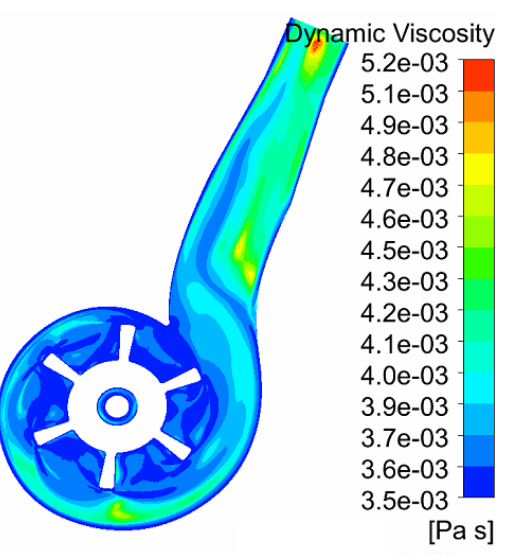

(f) Vorticity.

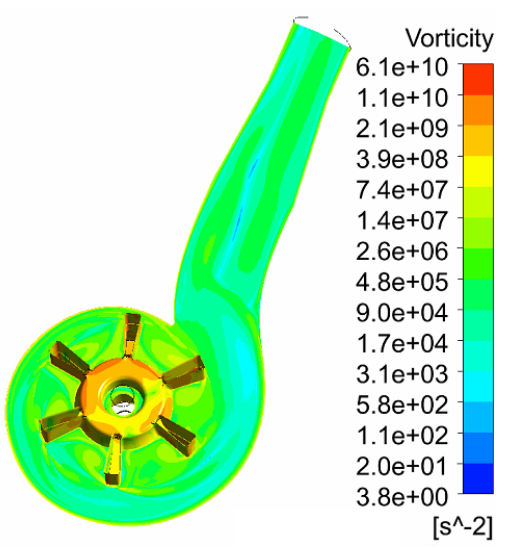

(h) Shear Stress (Volute).

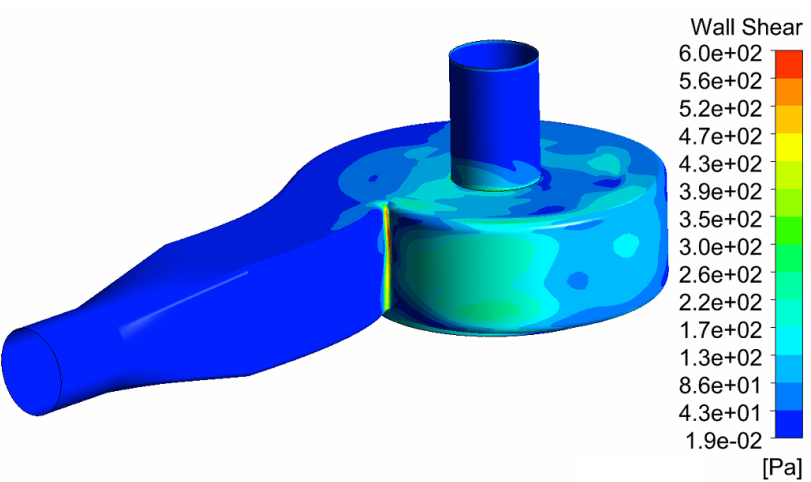

By analysing the wall shear stress in the impellers (Figs. 10.11g and 10.12g) it is possible to notice that the peak values occur at the blade inlet. The optimized impeller shows a peak value $(700[\mathrm{~Pa}])$ approximately $22 \%$ lower than the SB case $(900[\mathrm{~Pa}])$. For the optimized impeller, most of the distribution is between 0 and $200[P a]$ while the SB impeller distribution is between 0 and $260[P a]$. For both impellers, these values are higher than the ones shown in the previous section. Thus, as the mass flow and rotation increase 
the shear stress values increase as well.

The wall shear stress values at the volute walls is a relatively higher in the SB case (Fig. $10.12 \mathrm{~h}$ ) than in the optimized case (Fig. 10.11h). An interesting finding is that the shear stress peak values in the volute plots do not increase at the same rate as in the impeller plots with the change of operation point. This indicates that the volute optimization is more robust than the impeller optimization, and the local minimum can attend a wider range of operation points if the wall shear stress is the focus.

The functional values for both cases are shown in Tab. 10.2. As in the previous section, the values are split by domain, i.e., the impeller values are computed by integrating the functional in the impeller volume, and the volute values in the volute volume. The total values refer to the sum of the impeller and volute functionals.

It is possible to identify in Tab. 10.2 that the operation at $5[\mathrm{~L} / \mathrm{min}]$ presents higher values of energy dissipation and vorticity when compared with the operation at $3[\mathrm{~L} / \mathrm{min}]$ (Tab. 10.1), what is expected given that higher rotations are needed to achieve this operation point.

In the impeller domain, the optimized case demonstrate an overall improvement around $23 \%$, for both energy dissipation and vorticity. For the volute domain, this improvement falls to $17 \%$. When the entire domain is considered, the improvement is around $20 \%$. This consistent improvement shows that the designs optimized at $3[\mathrm{~L} / \mathrm{min}]$ and $3000[\mathrm{rpm}]$ can be used at near operational points. However, the best performance still is obtained at the design point. This indicates that each operation point has a local minimum. 
Table 10.2 - Functional values Straight-bladed and Optimized impellers operating with blood at $5[\mathrm{~L} / \mathrm{min}]$ and $100[\mathrm{mmHg}]$.

\begin{tabular}{llccc}
\hline & & Straight Blade & Optimized & Difference (\%) \\
\hline Mass Flow & {$[\mathrm{L} / \mathrm{min}]$} & 5 & 5 & - \\
Rotation & {$[\mathrm{rpm}]$} & 3200 & 3400 & $6 \%$ \\
Pressure & {$[\mathrm{mmHg}]$} & 101 & 100 & $-1 \%$ \\
& & & & \\
Impeller & & & & $-23 \%$ \\
Energy Dissip. & {$[\mathrm{W}]$} & 0.48 & 0.37 & $-23 \%$ \\
Vorticity & {$\left[\mathrm{m}^{3} / \mathrm{s}^{2}\right]$} & 138 & 106 & \\
& & & & $-17 \%$ \\
Volute & & & & $-17 \%$ \\
Energy Dissip. & {$[\mathrm{W}]$} & 0.57 & 0.47 & \\
Vorticity & {$\left[\mathrm{m}^{3} / \mathrm{s}^{2}\right]$} & 160 & 133 & $-20 \%$ \\
& & & & $-20 \%$ \\
Total & & & & 0.84 \\
Energy Dissip. & {$[\mathrm{W}]$} & 1.05 & 239 & \\
Vorticity & {$\left[\mathrm{m}^{3} / \mathrm{s}^{2}\right]$} & 297 & & \\
\hline
\end{tabular}




\section{CONCLUSION}

The concept of topology optimization (TO) is applied to the design of a VAD's volute and impeller. The main objective is to decrease the hemolysis ratio by minimizing energy dissipation. The literature shows that hemolysis has a high correlation with shear stress. The shear stress is directly proportional to the shear rate and therefore, to the viscous energy dissipation. Hence, minimizing energy dissipation should create better hematological conditions.

Initially, the turbulence algorithm behavior is analyzed by using an arbitrary Newtonian fluid. The impeller design considering turbulent flows shows that the design is very dependent on the model for high Reynolds numbers. For low Reynolds numbers, both N-S and RANS-SA optimized blades have better functional values than the initial curved blade, even though they have significantly different topologies. The different achieved minima for the RANS-SA can be associated with the extra weight brought by the additional viscosity in the energy dissipation functional. In the turbulent cases, the post-processed smoothed blades with the cross model check, show that the topologies obtained with the RANS-SA have a better performance. Hence, the RANS-SA could be used at low Reynolds numbers and should be used for high Reynolds numbers, given that the order of magnitude of the difference in the functional value is much more expressive.

The topology optimization of the non-Newtonian laminar impeller is analyzed. The results, for the proposed boundary conditions, shows that the topologies are not very dependent on the angular size of the domain, and the resulting topology varies with the parameters of the material model. By comparing the optimized blades with straight blades, we notice that it is possible to achieve energy dissipation values close to the values of the optimized blades by rotating the straight blade. However, the optimized blade is still better for both the energy dissipation and vorticity functionals.

The introduction of the Spalart-Allmaras turbulence model to the non-Newtonian model increases the effective viscosity of the model. The additional viscosity given by the eddy viscosity causes small changes in the curvature. This effect can be explained by the turbulent viscosity calculated by the model influencing the objective function and the algorithm.

The same methodology is applied to the volute design. The conclusions are very similar to the impeller conclusions. The turbulence model shows small influence in the algorithm when compared with the laminar non-Newtonian results. The final topologies are similar, with slight changes in their curvature near the outlet. 
Experimental essays are performed with the optimized impeller considering the non-Newtonian turbulent model. From the prior experience of the laboratory, the pressure by mass flow curve measured for the blood analog fluid is close to that obtained for water. Thus, the experimental results shown in this work used water as the fluid. These results are used to calibrate a 3D simulation of the complete model, so the flow inside the VAD can be further analyzed.

The experimental results are coherent with the computational model, implying that the analysis made for the model represents the prototype behavior. Hence, the shear rate, energy dissipation, and vorticity could be analyzed. The optimized impeller presents energy dissipation values around 40\% lower than the straight-bladed impeller's when operating with water. Also, from the point-wise plots of the shear rate and energy dissipation, the direct proportionality of these fields is verified. The integrated values over the volume of the energy dissipation and vorticity, reveal the strong correlation of these two functionals, given that the shape of the curves is almost identical.

Even though no experiment is done with blood, the computational model verified with water is used to simulate the operation with blood at $3[\mathrm{~L} / \mathrm{min}]$ and $3000[\mathrm{rpm}]$. The additional viscosity given by the non-Newtonian model modifies the calculated fields and the functional values. The optimized blade shows higher viscosity at the blades, which indicates lower velocity gradients. The optimized case shows lower peak (and integrated) values for the energy dissipation and vorticity, appointing to lower hemolysis. The wall shear stress analysis confirms this conclusion, given that the values are higher in the straight-bladed VAD.

An interesting fact is that the volute energy dissipation, vorticity, and wall shear stress distributions are affected by the impeller design. The integrated values on the volute volume are lower when the optimized impeller is used. This is a result of choosing the correct velocity angle when optimizing the volute. Thus, it is essential to choose the correct impeller and volute pair to achieve the best performance, and ideally, both should be optimized together in a coupled manner.

Aiming to verify the VAD operation as an adult VAD, simulations of the complete VAD operating with blood at $5[\mathrm{~L} / \mathrm{min}]$ and $100[\mathrm{mmHg}]$ are done. The rotation speed is increased following the similarity laws to achieve this operation point. The energy dissipation, vorticity, and shear stress values increase with the higher rotations. The improvement of $32 \%$ observed in the simulation at $3[\mathrm{~L} / \mathrm{min}]$ is reduced to around $20 \%$ with the higher mass flow and rotation. This shows that each operation point has a local minimum. However, the optimization is robust enough to maintain better functional values than the straight-bladed case for operation points close to the design point.

Finally, the proposed process of applying topology optimization to design ventricular 
assist device impellers and volutes defined by modeling, optimizing, post-processing, 3D printing prototypes, and performing the experimental characterization has been successfully implemented.

\subsection{Future Work}

Based on the aforementioned conclusions the author suggests that future work should focus on performing the optimization by using 3D models. The 3D model can represent better the turbulent behavior, given that turbulence is intrinsically a $3 \mathrm{D}$ phenomenon. Also, more advanced turbulence models should be used at the optimization phase of the VAD model, such as the $k-\omega S S T$ or the $k-\epsilon R N G$. These more complete models are indicated in the literature to better capture the turbulence behavior of blood flows.

Besides, considering other applications, it is important to include models that contemplate higher Reynolds numbers and compressible flows, for example, to design gas turbines and compressors. 


\section{REFERENCES}

AAROnSON, K. D.; SLAUGHTER, M. S.; MILLER, L. W.; MCGEE, E. C.; COTTS, W. G.; ACKER, M. a.; JESSUP, M. L.; GREGORIC, I. D.; LOYALKA, P.; FRAZIER, O. H.; JEEVANANDAM, V.; ANDERSON, A. S.; KORMOS, R. L.; TEUTEBERG, J. J.; LEVY, W. C.; NAFTEL, D. C.; BITTMAN, R. M.; PAGANI, F. D.; HATHAWAY, D. R.; BOYCE, S. W. Use of an Intrapericardial, Continuous-Flow, Centrifugal Pump in Patients Awaiting Heart Transplantation. Circulation, v. 125, n. 25, p. 3191-3200, jun 2012. ISSN 0009-7322. Available from Internet: <http://circ.ahajournals.org/cgi/doi/10.1161/CIRCULATIONAHA.111.058412>. Cited in page 19 .

ABRAHAM, F.; BEHR, M.; HEINKENSCHLOSS, M. The effect of stabilization in finite element methods for the optimal boundary control of the Oseen equations. Finite Elements in Analysis and Design, v. 41, n. 3, p. 229-251, dec 2004. ISSN 0168874X. Available from Internet: <http://linkinghub.elsevier.com/retrieve/pii/ S0168874X04000861>. Cited in page 51.

ABRAHAM, F.; BEHR, M.; HEINKENSCHLOSS, M. Shape optimization in steady blood flow: A numerical study of non-Newtonian effects. Computer Methods in Biomechanics and Biomedical Engineering, v. 8, n. 2, p. 127-137, 2005. ISSN 10255842. Cited 3 times in pages 31 , 44, and 50 .

ALLMARAS, S. R.; JOHNSON, F. T.; SPALART, P. R. Modifications and clarifications for the implementation of the Spalart-Allmaras turbulence model. Seventh International Conference on Computational Fluid Dynamics, n. ICCFD7-1902, p. 1-11, 2012. Available from Internet: <http://www.iccfd.org/iccfd7/assets/pdf/papers/ ICCFD7-1902\{\_\}paper.> Cited in page 37.

ALNAES, M.; LOGG, A.; MARDAL, K. A.; SKAVHAUG, O.; LANGTANGEN, H. Unified framework for finite element assembly. International Journal of Computational Science and Engineering, v. 4, n. 4, p. 231, may 2009. ISSN 1742-7185. Available from Internet: <http://arxiv.org/abs/1205.3039http://dx.doi.org/10.1504/IJCSE.2009. 029160http://www.inderscience.com/link.php?id=29160>. Cited in page 57 .

ALNæS, M. S.; LOGG, A.; ØLGAARD, K. B.; ROGNES, M. E.; WELLS, G. N. Unified form language. ACM Transactions on Mathematical Software, v. 40, n. 2, p. 1-37, mar 2014. ISSN 00983500. Available from Internet: <http://arxiv.org/abs/1005.3014http: //arxiv.org/abs/1211.4047http://dl.acm.org/citation.cfm?doid=2594412.2566630>. Cited in page 57.

ALONSO, D. H.; SÁ, L. F. N. de; SAENZ, J. S. R.; SILVA, E. C. N. Topology optimization applied to the design of 2D swirl flow devices. Structural and Multidisciplinary Optimization, v. 58, n. 6, p. 2341-2364, dec 2018. ISSN 1615-147X. Available from Internet: <http://link.springer.com/10.1007/s00158-018-2078-0>. Cited in page 27.

ALOnSO, D. H.; SÁ, L. F. N. de; SAENZ, J. S. R.; SILVA, E. C. N. Topology optimization based on a two-dimensional swirl flow model of Tesla-type pump devices. Computers \& Mathematics with Applications, v. 77, n. 9, p. 2499-2533, may 2019. 
ISSN 08981221. Available from Internet: <https://linkinghub.elsevier.com/retrieve/pii/ S0898122118307338>. Cited in page 27 .

AMESTOY, P. R.; DUFF, I. S.; L'EXCELLENT, J.-Y.; KOSTER, J. A Fully Asynchronous Multifrontal Solver Using Distributed Dynamic Scheduling. SIAM Journal on Matrix Analysis and Applications, v. 23, n. 1, p. 15-41, jan 2001. ISSN 0895-4798. Available from Internet: <http://epubs.siam.org/doi/abs/10.1137/S0895479899358194>, Cited in page 58

ANTAKI, J. F.; GHATTAS, O.; BURGREEN, G. W.; HE, B. Computational Flow Optimization of Rotary Blood Pump Components. Artificial Organs, v. 19, n. 7, p. 608-615, jul 1995. ISSN 0160564X. Available from Internet: <http://www.ncbi.nlm.nih. gov/pubmed/8572960http://doi.wiley.com/10.1111/j.1525-1594.1995.tb02389.x>. Cited 3 times in pages 21, 22, and 51 .

BALAY, S.; ABHYANKAR, S.; ADAMS, M. F.; BROWN, J.; BRUNE, P.; BUSCHELMAN, K.; DALCIN, L.; DENER, A.; EIJKHOUT, V.; GROPP, W. D.; KAUSHIK, D.; KNEPLEY, M. G.; MAY, D. A.; MCINNES, L. C.; MILLS, R. T.; MUNSON, T.; RUPP, K.; SANAN, P.; SMITH, B. F.; ZAMPINI, S.; ZHANG, H.; ZHANG, H. PETSc Web page. 2018. <http://www.mcs.anl.gov/petsc $>$. Available from Internet: <http://www.mcs.anl.gov/petsc $>$. Cited in page 57.

BALONI, B. D.; PATHAK, Y.; CHANNIWALA, S. Centrifugal blower volute optimization based on Taguchi method. Computers \& Fluids, Elsevier Ltd, v. 112, n. February, p. 72-78, may 2015. ISSN 00457930. Available from Internet: $<$ http://linkinghub.elsevier.com/retrieve/pii/S0045793015000444>. Cited 2 times in pages 20 and 90 .

BARRETT, K.; BROOKS, H.; BOITANO, S.; BARMAN, S. Ganong's review of medical physiology. [S.l.]: The McGraw-Hill Companies, Inc., 2010. 593 p. ISBN 978-0-07-160567-0. Cited 2 times in pages 23 and 69.

BEHBAHANI, M.; BEHR, M.; HORMES, M.; STEINSEIFER, U.; ARORA, D.; CORONADO, O.; PASQUALI, M. A review of computational fluid dynamics analysis of blood pumps. European Journal of Applied Mathematics, v. 20, n. 04, p. 363, aug 2009. ISSN 0956-7925. Available from Internet: <http: //www.journals.cambridge.org/abstract $\left\{\backslash \_\right.$\}S0956792509007>. Cited in page 21.

BENSON, S. J.; MUNSON, T. S. Flexible complementarity solvers for large-scale applications. Optimization Methods and Software, v. 21, n. 1, p. 155-168, feb 2006. ISSN 1055-6788. Available from Internet: <http://link.springer.com/10.1007/ s10589-005-3908-8http://www.tandfonline.com/doi/abs/10.1080/10556780500065382> Cited in page 57.

BERIS, P.; PICARD, V. Non-immune Hemolysis: Diagnostic Considerations. Seminars in Hematology, v. 52, n. 4, p. 287-303, oct 2015. ISSN 00371963. Available from Internet: <https://linkinghub.elsevier.com/retrieve/pii/S003719631500061X>. Cited in page 22

BIRATI, E. Y.; RAME, J. E. Diagnosis and Management of LVAD Thrombosis. Current Treatment Options in Cardiovascular Medicine, v. 17, n. 2, p. 2, feb 2015. ISSN 1092-8464. Available from Internet: <http://link.springer.com/10.1007/ s11936-014-0361-y>. Cited in page 22. 
BORRVALL, T.; PETERSSON, J. Topology optimization of fluids in Stokes flow. International Journal for Numerical Methods in Fluids, v. 41, n. 1, p. 77-107, jan 2003. ISSN 0271-2091. Available from Internet: <http://onlinelibrary.wiley.com/doi/ 10.1002/fld.426/abstracthttp://doi.wiley.com/10.1002/fld.426>. Cited 3 times in pages 43, 45, and 49.

BUEnO-OROVIO, A.; CASTRO, C.; PAlACIOS, F.; ZUAZUA, E. Continuous Adjoint Approach for the Spalart-Allmaras Model in Aerodynamic Optimization. AIAA Journal, v. 50, n. 3, p. 631-646, mar 2012. ISSN 0001-1452. Available from Internet: <http://arc.aiaa.org/doi/10.2514/1.J051307>. Cited in page 34.

CASAS, V.; PENA, F.; DURO, R. Automatic Design and Optimization of Wind Turbine Blades. In: 2006 International Conference on Computational Inteligence for Modelling Control and Automation and International Conference on Intelligent Agents Web Technologies and International Commerce (CIMCA'06). IEEE, 2006. p. 205-205. ISBN 0-7695-2731-0. Available from Internet: $<$ http://ieeexplore.ieee.org/lpdocs/epic03/wrapper.htm?arnumber=4052821>. Cited in page 20 .

CHIU, W.-C.; SLEPIAN, M. J.; BLUESTEIN, D. Thrombus Formation Patterns in the HeartMate II Ventricular Assist Device. ASAIO Journal, v. 60, n. 2, p. 237-240, 2014. ISSN 1058-2916. Available from Internet: <https://insights.ovid.com/crossref?an= 00002480-201403000-00017>. Cited in page 22 .

CHUONG, C.; FUNG, Y. Compressibility and constitutive equation of arterial wall in radial compression experiments. Journal of Biomechanics, v. 17, n. 1, p. 35-40, jan 1984. ISSN 00219290. Available from Internet: <https: //linkinghub.elsevier.com/retrieve/pii/0021929084900770>. Cited in page 30.

COLVIN-ADAMS, M.; SMITH, J. M.; HEUBNER, B. M.; SKEANS, M. A.; EDWARDS, L. B.; WALlER, C. D.; CALLAHAN, E. R.; SNYDER, J. J.; ISRANI, A. K.; KASISKE, B. L. OPTN/SRTR 2013 Annual Data Report: heart. American journal of transplantation : official journal of the American Society of Transplantation and the American Society of Transplant Surgeons, v. 15, p. 1-28, 2015. ISSN 16006143. Cited in page 18 .

DENG, Y.; LIU, Z.; WU, J.; WU, Y. Topology optimization of steady Navier-Stokes flow with body force. Computer Methods in Applied Mechanics and Engineering, Elsevier B.V., v. 255, p. 306-321, mar 2013. ISSN 00457825. Available from Internet: <http://linkinghub.elsevier.com/retrieve/pii/S0045782512003532>. Cited in page 43.

DERAKHSHAN, S.; POURMAHDAVI, M.; ABDOLAHNEJAD, E.; REIHANI, A.; OJAGHI, A. Numerical shape optimization of a centrifugal pump impeller using artificial bee colony algorithm. Computers \& Fluids, v. 81, p. 145-151, 2013. ISSN 00457930. Available from Internet: <http://linkinghub.elsevier.com/retrieve/pii/ S0045793013001588>. Cited 2 times in pages 20 and 23.

DILGEN, C. B.; DILGEN, S. B.; FUHRMAN, D. R.; SIGMUND, O.; LAZAROV, B. S. Topology optimization of turbulent flows. Computer Methods in Applied Mechanics and Engineering, Elsevier B.V., v. 331, p. 363-393, 2018. ISSN 00457825. Available from Internet: <https://doi.org/10.1016/j.cma.2017.11.029>, Cited 2 times in pages 44 and 55 . 
DILGEN, S. B.; DILGEN, C. B.; FUHRMAN, D. R.; SIGMUND, O.; LAZAROV,

B. S. Density based topology optimization of turbulent flow heat transfer systems. Structural and Multidisciplinary Optimization, v. 57, n. 5, p. 1905-1918, may 2018. ISSN 1615-147X. Available from Internet: <http: //link.springer.com/10.1007/s00158-018-1967-6>. Cited in page 44.

EVGRAFOV, A. Topology optimization of slightly compressible fluids.

Doktorsavhandlingar vid Chalmers Tekniska Hogskola, v. 62, n. 1, p. 55-81, 2004. ISSN 0346718X. Cited in page 43.

EVGRAFOV, A. The Limits of Porous Materials in the Topology Optimization of Stokes Flows. Applied Mathematics and Optimization, v. 52, n. 3, p. 263-277, oct 2005. ISSN 0095-4616. Available from Internet: <http://link.springer.com/10.1007/ s00245-005-0828-z>. Cited in page 43 .

FARRELL, P. E.; HAM, D. A.; FUnKE, S. W.; ROGNES, M. E. Automated Derivation of the Adjoint of High-Level Transient Finite Element Programs.

SIAM Journal on Scientific Computing, v. 35, n. 4, p. C369-C393, jan 2013. ISSN 1064-8275. Available from Internet: <http://arxiv.org/abs/1204.5577http: //epubs.siam.org/doi/abs/10.1137/120873558>. Cited in page 58 .

FRASER, W. H. Flow Recirculation in Centrifugal Pumps. [S.l.]: TENTH TURBOMACHINERY SYMPOSIUM, 2001. Cited in page 50.

FUNKE, S. W.; FARRELL, P. E. A framework for automated PDE-constrained optimisation. arXiv preprint arXiv:1302.3894, feb 2013. Available from Internet: $<$ http://arxiv.org/abs/1302.3894>. Cited 2 times in pages 55 and 59 .

GERSBORG-HANSEN, A. Topology optimization of incompressible Newtonian flows at moderate Reynolds numbers. Tese (Doutorado), 2003. Available from Internet: <http://scholar.google.com/scholar?hl=en $\{\&\}$ btnG=Search $\{\&\} \mathrm{q}=$ intitle: Topology+optimization +of+incompressible+Newtonian+flows+at+moderate+ Reynolds+num>. Cited 2 times in pages 43 and 45 .

GERSBORG-HANSEN, a.; SIGMUND, O.; HABER, R. Topology optimization of channel flow problems. Structural and Multidisciplinary Optimization, v. 30, n. 3, p. 181-192, sep 2005. ISSN 1615-147X. Available from Internet: <http://link.springer.com/10.1007/s00158-004-0508-7>. Cited in page 43.

GHADIMI, B.; NEJAT, A.; NOURBAKHSH, S. A.; NADERI, N. Shape optimization of a centrifugal blood pump by coupling CFD with metamodel-assisted genetic algorithm. Journal of Artificial Organs, Springer Japan, v. 0, n. 0, p. 0, oct 2018. ISSN 1434-7229. Available from Internet: <http://dx.doi.org/10.1007/s10047-018-1072-zhttp: //link.springer.com/10.1007/s10047-018-1072-z>. Cited in page 90.

GHATTAS, O.; HE, B.; ANTAKI, J. Shape optimization of Navier-Stokes flows with application to optimal design of artificial heart components. [S.l.], 1995. Available from Internet: <http://arc.aiaa.org/doi/10.2514/6.1994-4387>, Cited 3 times in pages 22 , 49 , and 51 .

GIANLUCA, R.; FRANCESCO, S.; GIUSEPPE, F. Past and present of cardiocirculatory assist devices: a comprehensive critical review. Journal of Geriatric Cardiology, 
v. 9, n. 4, p. 389-400, jan 2013. ISSN 1671-5411. Available from Internet: $<$ http://pub.chinasciencejournal.com/article/getArticleRedirect.action?doiCode=10. 3724/SP.J.1263.2012.05281>. Cited in page 18 .

GÖLCÜ, M.; PANCAR, Y.; SEKMEN, Y. Energy saving in a deep well pump with splitter blade. Energy Conversion and Management, v. 47, n. 5, p. 638-651, mar 2006. ISSN 01968904. Available from Internet: <http: //linkinghub.elsevier.com/retrieve/pii/S0196890405001287>. Cited in page 20.

GUYTON, A. C.; HALL, J. E. Textbook of medical physiology. 11th. ed. [S.l.: s.n.], 2006. 1152 p. ISBN 0-7216-0240-1. Cited 2 times in pages 23 and 30 .

HAN, J.; TRUMBLE, D. Cardiac Assist Devices: Early Concepts, Current Technologies, and Future Innovations. Bioengineering, v. 6, n. 1, p. 18, feb 2019. ISSN 2306-5354. Available from Internet: <http://www.mdpi.com/2306-5354/6/1/18>. Cited in page 18

HANSEN, M. O. L. Aerodynamics of Wind Turbines. 2. ed. [S.l.]: Routledge, 2007. 208 p. ISBN 978-1844074389. Cited in page 19.

HEINRICH, M.; SCHWARZE, R. Genetic Algorithm Optimization of the Volute Shape of a Centrifugal Compressor. International Journal of Rotating Machinery, v. 2016, p. 1-13, 2016. ISSN 1023-621X. Available from Internet: <http://www.hindawi.com/journals/ijrm/2016/4849025/>. Cited in page 90.

HYUN, J.; WANG, S.; YANG, S. Topology optimization of the shear thinning non-Newtonian fluidic systems for minimizing wall shear stress. Computers \& Mathematics with Applications, Elsevier Ltd, v. 67, n. 5, p. 1154-1170, mar 2014. ISSN 08981221. Available from Internet: <http://dx.doi.org/10.1016/j.camwa.2013.12.013http: //linkinghub.elsevier.com/retrieve/pii/S0898122113007074>. Cited 2 times in pages 44 and 46 .

JAFARZADEH, B.; HAJARI, A.; ALISHAHI, M.; AKBARI, M. The flow simulation of a low-specific-speed high-speed centrifugal pump. Applied Mathematical Modelling, v. 35, n. 1, p. 242-249, jan 2011. ISSN 0307904X. Available from Internet: <http://linkinghub.elsevier.com/retrieve/pii/S0307904X10002271>. Cited in page 19 .

KHURSHID, H.; HOFFMANN, K. A. Development of a high-order solver for blood flow. Engineering with Computers, v. 31, n. 1, p. 51-71, jan 2015. ISSN 0177-0667. Available from Internet: <http://link.springer.com/10.1007/s00366-013-0324-z>. Cited in page 30 .

KIRKLIN, J. K.; NAFTEL, D. C.; KORMOS, R. L.; STEVENSON, L. W.; PAGANI, F. D.; MILLER, M. a.; ULISNEY, K. L.; BALDWIN, J. T.; YOUNG, J. B. Second INTERMACS annual report: More than 1,000 primary left ventricular assist device implants. Journal of Heart and Lung Transplantation, v. 29, n. 1, p. 1-10, 2010. ISSN 10532498. Cited in page 19 .

KOZIK, D. J.; PLUNKETT, M. D. Mechanical circulatory support. Organogenesis, v. 7, n. 1, p. 50-63, 2011. ISSN 15476278. Cited in page 18.

LAUNDER, B.; SPALDING, D. The numerical computation of turbulent flows. Computer Methods in Applied Mechanics and Engineering, 
v. 3, n. 2, p. 269-289, mar 1974. ISSN 00457825. Available from Internet: <http://linkinghub.elsevier.com/retrieve/pii/0045782574900292>. Cited in page 33 .

LEE, Y.-T.; AHUJA, V.; HOSANGADI, A.; SLIPPER, M. E.; MULVIHILL, L. P.; BIRKBECK, R.; COLEMAN, R. M. Impeller Design of a Centrifugal Fan with Blade Optimization. International Journal of Rotating Machinery, v. 2011, p. 1-16, 2011. ISSN 1023-621X. Available from Internet: <http://www.hindawi.com/journals/ijrm/2011/ $537824 />$. Cited in page 19 .

LEUPRECHT, A.; PERKTOLD, K. Computer Simulation of Non-Newtonian Effects on Blood Flow in Large Arteries. Computer Methods in Biomechanics and Biomedical Engineering, v. 4, n. 2, p. 149-163, jan 2001. ISSN 1025-5842. Available from Internet: <http://www.ncbi.nlm.nih.gov/pubmed/11264865http: //www.tandfonline.com/doi/abs/10.1080/10255840008908002>. Cited in page 31.

LEVERETT, L.; HELlUMS, J.; ALFREY, C.; LYNCH, E. Red Blood Cell Damage by Shear Stress. Biophysical Journal, Elsevier, v. 12, n. 3, p. 257-273, mar 1972. ISSN 00063495. Available from Internet: <http://dx.doi.org/10.1016/S0006-3495(72) 86085-5https://linkinghub.elsevier.com/retrieve/pii/S0006349572860855>. Cited in page 22 .

LI, D.; WU, Q.; JI, J.; LIU, S.; ZHANG, M.; ZHANG, Y. Hemolysis in a continuous-flow ventricular assist device with/without chamfer. Advances in Mechanical Engineering, v. 9, n. 4, p. 168781401769789, apr 2017. ISSN 1687-8140. Available from Internet: <http://journals.sagepub.com/doi/10.1177/1687814017697894>. Cited in page 22 .

LOGG, A.; WELLS, G. N. DOLFIN. ACM Transactions on Mathematical Software, v. 37, n. 2, p. 1-28, apr 2010. ISSN 00983500. Available from Internet: $<$ http://portal.acm.org/citation.cfm?doid $=1731022.1731030>$. Cited in page 57 .

LOGG, A.; WELLS, G. N.; BOOK, T. F. Automated Solution of Differential Equations by the Finite Element Method. Berlin, Heidelberg: Springer Berlin Heidelberg, 2012. v. 84. ISBN 978-3-642-23098-1. Available from Internet: <http://link.springer.com/10.1007/978-3-642-23099-8>. Cited in page 57 .

LYONS, S. L.; HANRATTY, T. J.; MCLAUGHLIN, J. B. Large Sscale computer simulation of fully developed turbulent channel flow with heat transfer. International Journal for Numerical Methods in Fluids, v. 13, n. 8, p. 999-1028, 1991. ISSN 10970363. Cited in page 32 .

MACOSKO, C. W. Rheology: Principles, Measurements and Applications. [S.l.]: Wiley, 1994. 568 p. ISBN 978-0-471-18575-8. Cited in page 31.

MANN, D. L.; ZIPES, D. P.; LIBBY, P.; BONOW, R. O.; BRAUNWALD, E. Braunwald's Heart Disease: A Textbook of Cardiovascular Medicine. In: Elsevier Saunders. [S.l.: s.n.], 2015. p. 155-178. ISBN 9781437703986. Cited in page 18.

MCCAUGHEY, E. J.; VECELLIO, E.; LAKE, R.; LI, L.; BURNETT, L.; CHESHER, D.; BRAYE, S.; MACKAY, M.; GAY, S.; BADRICK, T.; WESTBROOK, J.; GEORGIOU, A. Key factors influencing the incidence of hemolysis: A critical appraisal of current evidence. Critical Reviews in Clinical Laboratory Sciences, Informa Healthcare USA, Inc, v. 54, n. 1, p. 59-72, jan 2017. ISSN 1040-8363. 
Available from Internet: <http://dx.doi.org/10.1080/10408363.2016.1250247https: //www.tandfonline.com/doi/full/10.1080/10408363.2016.1250247>. Cited in page 22.

MCMURRAY, J. J. V.; ADAMOPOULOS, S.; ANKER, S. D.; AURICCHIO, A.; BOHM, M.; DICKSTEIN, K.; FALK, V.; FILIPPATOS, G.; FONSECA, C.; GOMEZ-SANCHEZ, M. A.; JAARSMA, T.; KOBER, L.; LIP, G. Y. H.; MAGGIONI, A. P.; PARKHOMENKO, A.; PIESKE, B. M.; POPESCU, B. A.; RONNEVIK, P. K.; RUTTEN, F. H.; SCHWITTER, J.; SEFEROVIC, P.; STEPINSKA, J.; TRINDADE, P. T.; VOORS, A. A.; ZANNAD, F.; ZEIHER, A.; BAX, J. J.; BAUMGARTNER, H.; CECONI, C.; DEAN, V.; DEATON, C.; FAGARD, R.; FUNCK-BRENTANO, C.; HASDAI, D.; HOES, A.; KIRCHHOF, P.; KNUUTI, J.; KOLH, P.; MCDONAGH, T.; MOULIN, C.; POPESCU, B. A.; REINER, Z.; SECHTEM, U.; SIRNES, P. A.; TENDERA, M.; TORBICKI, A.; VAHANIAN, A.; WINDECKER, S.; MCDONAGH, T.; SECHTEM, U.; BONET, L. A.; AVRAAMIDES, P.; Ben Lamin, H. A.; BRIGNOLE, M.; COCA, A.; COWBURN, P.; DARGIE, H.; ELLIOTT, P.; FLACHSKAMPF, F. A.; GUIDA, G. F.; HARDMAN, S.; IUNG, B.; MERKELY, B.; MUELLER, C.; NANAS, J. N.; NIELSEN, O. W.; ORN, S.; PARISSIS, J. T.; PONIKOWSKI, P. ESC Guidelines for the diagnosis and treatment of acute and chronic heart failure 2012: The Task Force for the Diagnosis and Treatment of Acute and Chronic Heart Failure 2012 of the European Society of Cardiology. Developed in collaboration with the Heart. European Heart Journal, v. 33, n. 14, p. 1787-1847, jul 2012. ISSN 0195-668X. Available from Internet: $<$ https://academic.oup.com/eurheartj/article-lookup/doi/10.1093/eurheartj/ehs104>. Cited in page 18.

MONTEVECCHI, F.; INZOLI, F.; REDAELLI, A.; MAMMANA, M. Preliminary Design and Optimization of an ECC Blood Pump by Means of a Parametric Approach. Artificial Organs, v. 19, n. 7, p. 685-690, jul 1995. ISSN 0160564X. Available from Internet: <http://doi.wiley.com/10.1111/j.1525-1594.1995.tb02403.x>. Cited in page 23

MOSER, R. D.; KIM, J.; MANSOUR, N. N. Direct numerical simulation of turbulent channel flow up to $\operatorname{Re} \tau=590$. Physics of Fluids, v. 11, n. 4, p. 943-945, apr 1999. ISSN 1070-6631. Available from Internet: <http://aip.scitation.org/doi/10.1063/1.869966>. Cited in page 32

OLESEN, L. H.; OKKELS, F.; BRUUS, H. A high-level programming-language implementation of topology optimization applied to steady-state Navier-Stokes flow. International Journal for Numerical Methods in Engineering, v. 65, n. 7, p. 975-1001, feb 2006. ISSN 0029-5981. Available from Internet: <http://doi.wiley.com/10.1002/nme.1468>. Cited in page 43 .

OTHMER, C. A continuous adjoint formulation for the computation of topological and surface sensitivities of ducted flows. International Journal for Numerical Methods in Fluids, v. 58, n. 8, p. 861-877, nov 2008. ISSN 02712091. Available from Internet: <http://doi.wiley.com/10.1002/fld.1770>. Cited 2 times in pages 53 and 54 .

PAPOUTSIS-KIACHAGIAS, E. M.; GIANNAKOGLOU, K. C. Continuous Adjoint Methods for Turbulent Flows, Applied to Shape and Topology Optimization:

Industrial Applications. Archives of Computational Methods in Engineering, Springer Netherlands, v. 23, n. 2, p. 255-299, jun 2016. ISSN 1134-3060. Available from Internet: <http://dx.doi.org/10.1007/s11831-014-9141-9http: //link.springer.com/10.1007/s11831-014-9141-9>. Cited 2 times in pages 26 and 55. 
PINGEN, G.; MAUTE, K. Optimal design for non-Newtonian flows using a topology optimization approach. Computers \& Mathematics with Applications, Elsevier Ltd, v. 59, n. 7, p. 2340-2350, apr 2010. ISSN 08981221. Available from Internet: <http://dx.doi.org/10.1016/j.camwa.2009.08.044http://linkinghub.elsevier.com/retrieve/ pii/S0898122109006336>, Cited 3 times in pages 26, 43, and 46.

PROBST, M.; LÜLFESMANN, M.; NICOLAI, M.; BÜCKER, H.; BEHR, M.; BISCHOF, C. Sensitivity of optimal shapes of artificial grafts with respect to flow parameters.

Computer Methods in Applied Mechanics and Engineering, Elsevier B.V., v. 199, n. 17-20, p. 997-1005, mar 2010. ISSN 00457825. Available from Internet: <http://dx.doi.org/10.1016/j.cma.2009.11.013http://linkinghub.elsevier.com/retrieve/ pii/S0045782509003880>. Cited in page 50.

QUARTERONI, A.; ROZZA, G. Optimal Control and Shape Optimization of Aorto-Coronaric Bypass Anastomoses. Mathematical Models and Methods in Applied Sciences, v. 13, n. 12, p. 1801-1823, 2003. ISSN 0218-2025. Cited in page 51.

RAVICHANDRAN, A. K.; PARKER, J.; NOVAK, E.; JOSEPH, S. M.; SCHILLING, J. D.; EWALD, G. A.; SILVESTRY, S. Hemolysis in left ventricular assist device: A retrospective analysis of outcomes. The Journal of Heart and Lung Transplantation, Elsevier, v. 33, n. 1, p. 44-50, jan 2014. ISSN 10532498. Available from Internet: <http://dx.doi.org/10.1016/j.healun.2013.08.019https: //linkinghub.elsevier.com/retrieve/pii/S1053249813014150>. Cited in page 22.

ROBERTSON, A. M.; SEQUEIRA, A.; OWENS, R. G. Rheological models for blood. Milano: Springer Milan, 2009. 211-241 p. Available from Internet: $<$ http://link.springer.com/10.1007/978-88-470-1152-6\_6>. Cited 2 times in pages 30 and 31 .

RODI, W. Turbulent Models and Their Application in Hydraulics-A State of Art Review. Taylor \& Francis, 1993. ISBN 9789054101505. Available from Internet: <https://books.google.com.br/books?id=7fIADgU8gp4C>. Cited in page 33 .

ROMERO, J. S.; N. Silva, E. C. Non-newtonian laminar flow machine rotor design by using topology optimization. Structural and Multidisciplinary Optimization, Structural and Multidisciplinary Optimization, v. 55, n. 5, p. 1711-1732, may 2017. ISSN 1615-147X. Available from Internet: <http://link.springer.com/10.1007/s00158-016-1599-7>, Cited 5 times in pages 21, 22, 26, 44, and 51.

ROMERO, J. S.; SILVA, E. C. N. A topology optimization approach applied to laminar flow machine rotor design. Comput. Methods Appl. Mech. Engrg, v. 279, p. 268-300, 2014. Available from Internet: <www.elsevier.com/locate/cma $>$. Cited 4 times in pages $29,30,40$, and 44 .

SÁ, L. F. N.; NOVOTNY, A. A.; ROMERO, J. S.; N. Silva, E. C. Design optimization of laminar flow machine rotors based on the topological derivative concept. Structural and Multidisciplinary Optimization, v. 56, n. 5, p. 1013-1026, nov 2017. ISSN 1615-147X. Available from Internet: <http://link.springer.com/10.1007/s00158-017-1698-0>. Cited 2 times in pages 27 and 44 .

SÁ, L. F. N.; R. Amigo, R. C.; NOvOtny, A. A.; N. Silva, E. C. Topological derivatives applied to fluid flow channel design optimization problems. Structural and 
Multidisciplinary Optimization, v. 54, n. 2, p. 249-264, aug 2016. ISSN 1615-147X. Available from Internet: <http://link.springer.com/10.1007/s00158-016-1399-0>. Cited in page 43 .

SÁ, L. F. N.; ROMERO, J. S.; HORIKAWA, O.; SILVA, E. C. N. Topology optimization applied to the development of small scale pump. Structural and Multidisciplinary Optimization, v. 57, n. 5, p. 2045-2059, may 2018. ISSN 1615-147X. Available from Internet: <http://link.springer.com/10.1007/s00158-018-1966-7>. Cited 4 times in pages 27, 98,101 , and 102 .

SÁ, L. F. N. de. Topology optimization method applied to laminar flow machine rotor design. São Paulo: [s.n.], 2016. Available from Internet: <http://www.teses.usp.br/teses/disponiveis/3/3152/tde-16032017-103709/>. Cited in page 29 .

SELGRADE, B. P.; TRUSKEY, G. A. Computational Fluid Dynamics Analysis to Determine Shear Stresses and Rates in a Centrifugal Left Ventricular Assist Device. Artificial Organs, v. 36, n. 4, p. E89-E96, apr 2012. ISSN 0160564X. Available from Internet: <http://doi.wiley.com/10.1111/j.1525-1594.2011.01416.x>. Cited 2 times in pages 22 and 23 .

SILVA, A. da; KOBAYASHI, M.; COIMBRA, C. Optimal design of non-Newtonian, micro-scale viscous pumps for biomedical devices. Biotechnology and Bioengineering, v. 96, n. 1, p. 37-47, jan 2007. ISSN 00063592. Available from Internet: <http: //doi.wiley.com/10.1002/bit.21165>. Cited in page 21.

SILVA, A. da; KOBAYASHI, M.; COIMBRA, C. Optimal theoretical design of 2-D microscale viscous pumps for maximum mass flow rate and minimum power consumption. International Journal of Heat and Fluid Flow, v. 28, n. 3, p. 526-536, jun 2007. ISSN 0142727X. Available from Internet: <http: //linkinghub.elsevier.com/retrieve/pii/S0142727X06001457>. Cited in page 21.

SLAUGHTER, M. S.; PAGANI, F. D.; MCGEE, E. C.; BIRKS, E. J.; COTTS, W. G.; GREGORIC, I.; Howard Frazier, O.; ICENOGLE, T.; NAJJAR, S. S.; BOYCE, S. W.; ACKER, M. a.; JOHN, R.; HATHAWAY, D. R.; NAJARIAN, K. B.; AARONSON, K. D. HeartWare ventricular assist system for bridge to transplant: Combined results of the bridge to transplant and continued access protocol trial. The Journal of Heart and Lung Transplantation, Elsevier, v. 32, n. 7, p. 675-683, jul 2013. ISSN 10532498. Available from Internet: <http://dx.doi.org/10.1016/j.healun.2013.04.004http: //linkinghub.elsevier.com/retrieve/pii/S1053249813012084>. Cited in page 18.

SPALART, P. Strategies for turbulence modelling and simulations. International Journal of Heat and Fluid Flow, v. 21, n. 3, p. 252-263, jun 2000. ISSN 0142727X. Available from Internet: <http://linkinghub.elsevier.com/retrieve/pii/ S0142727X00000072>. Cited in page 33.

SPALART, P.; ALLMARAS, S. A one-equation turbulence model for aerodynamic flows. 30th Aerospace Sciences Meeting and Exhibit, American Institute of Aeronautics and Astronautics, Reston, Virigina, jan 1992. ISSN 00341223. Available from Internet: <http://arc.aiaa.org/doi/10.2514/6.1992-439>. Cited 2 times in pages 33 and 35. 
SPALART, P.; SHUR, M. On the sensitization of turbulence models to rotation and curvature. Aerospace Science and Technology, v. 1, n. 5, p. 297-302, jul 1997. ISSN 12709638. Available from Internet: <http://linkinghub.elsevier.com/retrieve/pii/ S1270963897900511>. Cited in page 37 .

STEIN, W.; JOYNER, D. SAGE: System for Algebra and Geometry Experimentation*. ACM SIGSAM Bulletin, Timely Communication, v. 39, n. 2, 2005. Available from Internet: <http://sigsam.org/bulletin/articles/152/sage.pdf $>$. Cited in page 58 .

STEPANOFF, A. Centrifugal and Axial Flow Pumps: Theory, Design, and Application. 2. ed. New York: Krieger Publishing Company, 1993. 540 p. Cited in page 91 .

STEWART, G. C.; GIVERTZ, M. M. Mechanical Circulatory Support for Advanced Heart Failure. Circulation, v. 125, n. 10, p. 1304-1315, mar 2012. ISSN 0009-7322. Available from Internet: <https://www.ahajournals.org/doi/10.1161/CIRCULATIONAHA.111.060830> Cited in page 18.

SVANBERG, K. The method of moving asymptotes - a new method for structural optimization. International Journal for Numerical Methods in Engineering, v. 24, n. 2, p. 359-373, feb 1987. ISSN 0029-5981. Available from Internet: <http://doi.wiley.com/10.1002/nme.1620240207>. Cited in page 59.

TANG, D.; YANG, C.; KOBAYASHI, S.; KU, D. N. Effect of a Lipid Pool on Stress/Strain Distributions in Stenotic Arteries: 3-D Fluid-Structure Interactions (FSI) Models.

Journal of Biomechanical Engineering, v. 126, n. 3, p. 363, 2004. ISSN 01480731.

Available from Internet: <http://biomechanical.asmedigitalcollection.asme.org/article. aspx?articleid $=1411833>$. Cited in page 103 .

TAO, R.; XIAO, R.; YANG, W.; WANG, F. A Comparative Assessment of Spalart-Shur Rotation/Curvature Correction in RANS Simulations in a Centrifugal Pump Impeller. Mathematical Problems in Engineering, v. 2014, p. 1-9, 2014. ISSN 1024-123X. Available from Internet: <http://www.hindawi.com/journals/mpe/2014/342905/>. Cited 2 times in pages 34 and 37.

TUCKER, P. G.; RUMSEY, C. L.; SPALART, P. R.; BARTELS, R. B.; BIEDRON, R. T. Computations of Wall Distances Based on Differential Equations. AIAA Journal, v. 43, n. 3, p. 539-549, mar 2005. ISSN 0001-1452. Available from Internet: <http://arc.aiaa.org/doi/10.2514/1.8626>, Cited in page 38.

URIEL, N.; HAN, J.; MORRISON, K. A.; NAHUMI, N.; YUZEFPOLSKAYA, M.; GARAN, A. R.; DUONG, J.; COLOMBO, P. C.; TAKAYAMA, H.; THOMAS, S.; NAKA, Y.; JORDE, U. P. Device thrombosis in HeartMate II continuous-flow left ventricular assist devices: A multifactorial phenomenon. The Journal of Heart and Lung Transplantation, Elsevier, v. 33, n. 1, p. 51-59, jan 2014. ISSN 10532498. Available from Internet: <http://dx.doi.org/10.1016/j.healun.2013.10.005https: //linkinghub.elsevier.com/retrieve/pii/S1053249813014629>. Cited in page 22.

WÄCHTER, A. An Interior Point Algorithm for Large-Scale Nonlinear Optimization with Applications in Process Engineering. Tese (Doutorado), 2002. Cited in page 59 . 
WÄCHTER, A. Short Tutorial: Getting Started With Ipopt in 90 Minutes. In: TOLEDO, U. N.; SCHENK, O.; SIMON, H. D.; SIVAN (Ed.). Combinatorial Scientific Computing. Dagstuhl, Germany: Schloss Dagstuhl - Leibniz-Zentrum fuer Informatik, Germany, 2009. ISBN 1862-4405. ISSN 1862-4405. Available from Internet: <http://drops.dagstuhl.de/opus/volltexte/2009/2089>. Cited in page 59.

WÄCHTER, A.; BIEGLER, L. T. On the implementation of an interior-point filter line-search algorithm for large-scale nonlinear programming. Mathematical Programming, v. 106, n. 1, p. 25-57, mar 2006. ISSN 0025-5610. Available from Internet: <http://link.springer.com/10.1007/s10107-004-0559-y>. Cited in page 59.

WALKER, A. M.; JOHNSTON, C. R.; RIVAL, D. E. On the Characterization of a Non-Newtonian Blood Analog and Its Response to Pulsatile Flow Downstream of a Simplified Stenosis. Annals of Biomedical Engineering, v. 42, n. 1, p. 97-109, jan 2014. ISSN 0090-6964. Available from Internet: <http://link.springer.com/10.1007/ s10439-013-0893-4>. Cited in page 103.

WEN-GUANG, L. Inverse Design of Impeller Blade of Centrifugal Pump with a Singularity Method. Jordan Journal of Mechanical and Industrial ..., v. 5, n. 2, p. 119-128, 2011. ISSN 19956665. Available from Internet: <http://jjmie.hu.edu.jo/files/v5n2/Binder1.pdf\#page=14>. Cited in page 20.

WHITE, F. White, F. (2010). Fluid Mechanics (7th ed.). McGraw-Hill Science/Engineering/Math.Fluid Mechanics. 7. ed. [S.l.]: McGraw-Hill Science/Engineering/Math, 2010. 862 p. ISBN 0077422414. Cited in page 51.

W.H.O., W. H. O. 2017. Available from Internet: <http://www.who.int/en/news-room/ fact-sheets/detail/cardiovascular-diseases-(cvds) $>$. Cited in page 18.

WIKER, N.; KLARBRING, A.; BORRVALL, T. Topology optimization of regions of Darcy and Stokes flow. International Journal for Numerical Methods in Engineering, v. 69, n. July 2006, p. 1374-1404, 2007. ISSN 00295981. Cited in page 43

WILCOX, D. C. Formulation of the k-w Turbulence Model Revisited. AIAA Journal, v. 46, n. 11, p. 2823-2838, nov 2008. ISSN 0001-1452. Available from Internet: $<$ http://arc.aiaa.org/doi/10.2514/1.36541>. Cited in page 33 .

YOON, G. H. Topology optimization for turbulent flow with Spalart-Allmaras model. Computer Methods in Applied Mechanics and Engineering, Elsevier B.V., v. 303, p. 288-311, may 2016. ISSN 00457825. Available from Internet: <http://dx.doi.org/10.1016/j.cma.2016.01.014http://linkinghub.elsevier.com/retrieve/ pii/S004578251630007X $>$, Cited 4 times in pages 26, 34, 44, and 46.

YU, S.; NG, B.; CHAN, W.; CHUA, L. The flow patterns within the impeller passages of a centrifugal blood pump model. Medical Engineering \& Physics, v. 22, n. 6, p. 381-393, jul 2000. ISSN 13504533. Available from Internet: <http://linkinghub.elsevier.com/retrieve/pii/S135045330000045X>. Cited in page 19 .

ZHANG, B.; LIU, X. Topology optimization study of arterial bypass configurations using the level set method. Structural and Multidisciplinary Optimization, v. 51, n. 3, p. 773-798, 2015. ISSN 16151488. Cited 2 times in pages 31 and 44. 
ZHANG, C. Optimization of Centrifugal Impellers for Uniform Discharge Flow and Wide Operating Range. Journal of Propulsion and Power, v. 28, n. 5, p. 888-899, sep 2012. ISSN 0748-4658. Available from Internet: <http://arc.aiaa.org/doi/10.2514/1.B34193> Cited in page 34 .

ZHANG, J.; ZHANG, P.; FRASER, K. H.; GRIFFITH, B. P.; WU, Z. J. Comparison and Experimental Validation of Fluid Dynamic Numerical Models for a Clinical Ventricular Assist Device. Artificial Organs, v. 37, n. 4, p. 380-389, apr 2013. ISSN 0160564X. Available from Internet: <http://doi.wiley.com/10.1111/j.1525-1594.2012.01576.x>. Cited 2 times in pages 34 and 38 .

ZYMARIS, A.; PAPADIMITRIOU, D.; GIANNAKOGLOU, K.; OTHMER, C. Continuous adjoint approach to the Spalart-Allmaras turbulence model for incompressible flows.

Computers \& Fluids, Elsevier Ltd, v. 38, n. 8, p. 1528-1538, sep 2009. ISSN 00457930. Available from Internet: <http://dx.doi.org/10.1016/j.compfluid.2008.12.006http: //linkinghub.elsevier.com/retrieve/pii/S0045793008002521>. Cited in page 34 . 


\section{APPENDIX A - CONTINUOUS ADJOINT DERIVATION}

In this chapter, the derivation of the continuous adjoint problem for each state equation is shown. The adjoint problem

\section{A.1 Navier-Stokes Adjoint Problem}

The adjoint equation for the Navier-Stokes problem is derived by considering the energy dissipation functional. Thus, we consider the following problem:

$$
\begin{aligned}
& \text { Minimize }: \quad \int_{\Omega} \frac{\mu}{2} \nabla \mathbf{u}: \nabla \mathbf{u} d \Omega+\int_{\Omega} \kappa(\alpha) \mathbf{u} \cdot \mathbf{u} d \Omega \\
& \mathbf{u}, p, \alpha
\end{aligned}
$$

subjected to :

$$
\begin{array}{ll}
\rho(\mathbf{u} \cdot \nabla) \mathbf{u}+\nabla p-\mu \nabla^{2} \mathbf{u}+\kappa(\alpha) \mathbf{u}=\mathbf{0} & \text { in } \Omega \\
\nabla \cdot \mathbf{u}=0 & \text { in } \Omega \\
\mathbf{u}=\mathbf{u}_{0} & \text { on } \partial \Omega_{\text {inlet }} \\
\mathbf{u}=\mathbf{0} & \text { on } \partial \Omega_{\text {wall }} \\
\nabla \mathbf{u} \cdot \mathbf{n}=p \mathbf{n} & \text { on } \partial \Omega_{\text {outlet }}
\end{array}
$$

Firstly the Lagrangian is defined:

$$
\begin{aligned}
L(\mathbf{u}, p, \alpha, \mathbf{a}, b, \mathbf{c}, \mathbf{d}, \mathbf{e})= & \int_{\Omega} \frac{\mu}{2} \nabla \mathbf{u}: \nabla \mathbf{u}+\kappa(\alpha) \mathbf{u} \cdot \mathbf{u} d \Omega \\
& -\int_{\Omega} \mathbf{a} \cdot\left[\rho(\mathbf{u} \cdot \nabla) \mathbf{u}+\nabla p-\mu \nabla^{2} \mathbf{u}+\kappa(\alpha) \mathbf{u}\right] d \Omega \\
& -\int_{\Omega} b[\nabla \cdot \mathbf{u}] d \Omega \\
& -\int_{\partial \Omega} \mathbf{c} \cdot\left[\mathbf{u}-\mathbf{u}_{0}\right] d \Gamma_{\text {inlet }} \\
& -\int_{\partial \Omega}^{\mathbf{d}} \cdot[\mathbf{u}-\mathbf{0}] d \Gamma_{\text {wall }} \\
& -\int_{\partial \Omega} \mathbf{e} \cdot[\nabla \mathbf{u} \cdot \mathbf{n}-p \mathbf{n}] d \Gamma_{\text {outlet }}
\end{aligned}
$$

where $\mathbf{a}, b, \mathbf{c}, \mathbf{d}$, e are the lagrange multipliers.

Then, the Fréchet derivative in the directions of $\mathbf{u}$ and $p$ to get the adjoint problem, notice that the derivative in the remaining directions $(\mathbf{a}, b, \mathbf{c}, \mathbf{d}, \mathbf{e})$ recover the constraints 
of the original problem (Eq. A.1). In the $\mathbf{u}$ direction it is given by:

$$
\begin{aligned}
L_{u}(\mathbf{u}, p, \alpha, \mathbf{a}, b, \mathbf{c}, \mathbf{d}, \mathbf{e} ; \mathbf{v})= & \int_{\Omega} \mu \nabla \mathbf{u}: \nabla \mathbf{v}+2 \kappa(\alpha) \mathbf{u} \cdot \mathbf{v} d \Omega \\
& -\int_{\Omega} \mathbf{a} \cdot\left[\rho(\nabla \mathbf{u} \cdot \mathbf{v}+\nabla \mathbf{v} \cdot \mathbf{u})-\mu \nabla^{2} \mathbf{v}+\kappa(\alpha) \mathbf{v}\right] d \Omega \\
& -\int_{\Omega} b[\nabla \cdot \mathbf{v}] d \Omega \\
& -\int_{\partial \Omega} \mathbf{c} \cdot \mathbf{v} d \Gamma_{\text {inlet }} \\
& -\int_{\partial \Omega}^{\mathbf{d} \cdot \mathbf{v} d \Gamma_{\text {wall }}} \\
& -\int_{\partial \Omega}^{\mathbf{e}} \cdot[\nabla \mathbf{v} \cdot \mathbf{n}] d \Gamma_{\text {outlet }}
\end{aligned}
$$

Integrating by parts the terms highlighted in red, in order,

$$
\begin{aligned}
L_{u 1} & =\int_{\Omega} \mu \nabla \mathbf{u}: \nabla \mathbf{v} d \Omega=\int_{\partial \Omega} \mu(\nabla \mathbf{u} \cdot \mathbf{n}) \cdot \mathbf{v} d \Gamma-\int_{\Omega} \mu \nabla^{2} \mathbf{u} \cdot \mathbf{v} d \Omega \\
L_{u 2} & =\int_{\Omega} \mathbf{a} \cdot \nabla \mathbf{v} \cdot \mathbf{u} d \Omega=\int_{\partial \Omega}(\mathbf{u} \cdot \mathbf{n}) \mathbf{a} \cdot \mathbf{v} d \Gamma-\int_{\Omega}(\nabla \mathbf{a} \cdot \mathbf{u}) \cdot \mathbf{v} d \Omega \\
L_{u 3} & =\int_{\Omega} \mu \mathbf{a} \cdot \nabla^{2} \mathbf{v} d \Omega=\mu \int_{\partial \Omega} \mathbf{a} \cdot(\nabla \mathbf{v} \cdot \mathbf{n}) d \Gamma-\mu \int_{\Omega} \nabla \mathbf{v}: \nabla \mathbf{a} d \Omega \\
& =\mu \int_{\partial \Omega} \mathbf{a} \cdot(\nabla \mathbf{v} \cdot \mathbf{n}) d \Gamma-\mu \int_{\partial \Omega}(\nabla \mathbf{a} \cdot \mathbf{n}) \cdot \mathbf{v} d \Gamma+\mu \int_{\Omega} \nabla^{2} \mathbf{a} \cdot \mathbf{v} d \Omega \\
L_{u 4} & =\int_{\Omega} b \nabla \cdot \mathbf{v} d \Omega=\int_{\partial \Omega} b \mathbf{v} \cdot \mathbf{n} d \Gamma-\int_{\Omega} \nabla b \cdot \mathbf{v} d \Omega
\end{aligned}
$$

The Fréchet derivative in the $p$ direction it is given by:

$$
\begin{aligned}
L_{p}(\mathbf{u}, p, \alpha, \mathbf{a}, b, \mathbf{c}, \mathbf{d}, \mathbf{e} ; q) & =-\int_{\Omega} \mathbf{a} \cdot \nabla q d \Omega-\int_{\partial \Omega} q \mathbf{e} \cdot \mathbf{n} d \Gamma_{\text {outlet }} \\
& =-\int_{\partial \Omega} q \mathbf{a} \cdot \mathbf{n} d \Gamma+\int_{\Omega} q \nabla \cdot \mathbf{a} d \Omega-\int_{\Omega} q \mathbf{e} \cdot \mathbf{n} d \Gamma_{\text {outlet }}
\end{aligned}
$$

Gathering the terms from $L_{u}$ with $\mathbf{v} d \Omega$ :

$$
\overbrace{-\mu \nabla^{2} \mathbf{u}}^{L_{u 1}}+2 \kappa(\alpha) \mathbf{u}-\rho \mathbf{a} \cdot \nabla \mathbf{u}+\overbrace{\rho \nabla \mathbf{a} \cdot \mathbf{u}}^{L_{u 2}}+\overbrace{\mu \nabla^{2} \mathbf{a}}^{L_{u 3}}-\kappa(\alpha) \mathbf{a}+\overbrace{\nabla b}^{L_{u 4}}=\mathbf{0}
$$

Gathering the terms from $L_{u}$ with $\mathbf{v} d \Gamma$ :

$$
\overbrace{\mu(\nabla \mathbf{u} \cdot \mathbf{n})}^{L_{u 1}}+\overbrace{(\mathbf{u} \cdot \mathbf{n}) \mathbf{a}}^{L_{u 2}}-\overbrace{\mu(\nabla \mathbf{a} \cdot \mathbf{n})}^{L_{u 3}}-\overbrace{b \mathbf{n}}^{L_{u 4}}=\mathbf{0}
$$

Gathering the terms from $L_{u}$ with $\nabla \mathbf{v} \cdot \mathbf{n} d \Gamma$, except at the outlet:

$$
\mathbf{a}=\mathbf{0}
$$


Reordering the terms and grouping the remaining terms:

$$
\begin{aligned}
-\mu \nabla^{2} \mathbf{a}+\rho\left(\nabla \mathbf{u}^{T} \cdot \mathbf{a}-\nabla \mathbf{a} \cdot \mathbf{u}\right)-\nabla b+\kappa(\alpha) \mathbf{a}=-\mu \nabla^{2} \mathbf{u}+2 \kappa(\alpha) \mathbf{u} & \text { in } \Omega \\
\nabla \cdot \mathbf{a}=0 & \text { in } \Omega \\
\mu(\nabla \mathbf{u} \cdot \mathbf{n})+(\mathbf{u} \cdot \mathbf{n}) \mathbf{a}-\mu(\nabla \mathbf{a} \cdot \mathbf{n})-b \mathbf{n}=\mathbf{0} & \text { on } \partial \Omega \\
\mathbf{a}=\mathbf{0} & \text { on } \partial \Omega_{\text {inlet } \cup \text { wall }} \\
\mathbf{a}=\mathbf{e} & \text { on } \partial \Omega_{\text {outlet }}
\end{aligned}
$$

the third equation should have more terms at the inlet and at the walls, however, when we consider the variational form to solve the complete system the third equation, which is a Robin condition, cancels with terms provident from the integration by parts. This would leave open valued, at the inlet contour, the variable $\mathbf{c}$ and, at the outlet contour, the variable $\mathbf{d}$. Thus, it is not necessary to put them in the strong formulation of the problem. 


\section{APPENDIX B - VERIFICATION OF THE IMPLEMENTED CODE}

In this appendix, the implementation for all cases (Newtonian, non-Newtonian, and turbulent) is verified by comparing the results of the FEniCS implementation with the ANSYS results. The resulting fields of velocity and dynamic viscosity are compared by interpolating them in a quadrilateral grid with Matlab and calculating the $L_{2}$ norm of the difference with respect to the mesh size, which represents the contribution of each element and can be written as,

$$
\|e\|_{L_{2}}=\left(\sum_{i=1}^{n_{e l}} h^{2}\left(\phi_{F E n i C S}-\phi_{A N S Y S}\right)^{2}\right)^{1 / 2}
$$

where $h$ is the mesh size (in one direction), $n_{e l}$ is the number of elements, and $\phi$ is the analysed variable.

In the following examples, a square mesh with sides equal to 1 and with a discretization of $200 \times 200$ elements is used. The fluid properties for the Newtonian cases are density equal to 1.0 and dynamic viscosity equal do 0.01, while for the non-Newtonian cases, the properties used are from the blood (Sec. 2.2. Two cases are analyzed for each model, one without rotation $(\omega=0[\mathrm{rpm}])$ and one with $\omega=100[\mathrm{rpm}]$. The boundary conditions are shown in Fig. B.1.

Figure B.1 - Boundary conditions for the verification of the implementation..

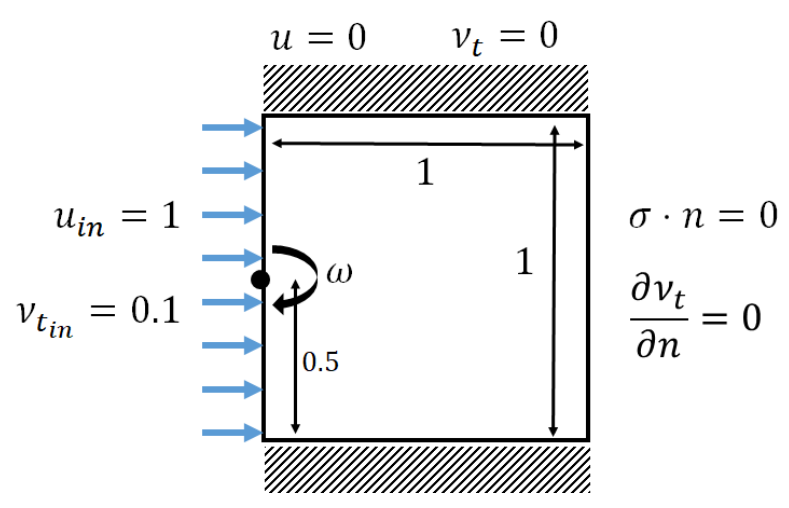




\section{B.1 Newtonian Flow}

The first case considers a laminar Newtonian flow in the channel proposed above. The variables plots are presented in Figs. B.2 and B.3, for $\omega=0$ and $\omega=100[\mathrm{rpm}]$, respectively. As we can see, for $\omega=0$ the fields present a very good agreement, with the highest differences appearing near the inlet. For $\omega=100$, the velocities difference is more noticeable; however, they are present in very few elements, and most of the difference field has a value lower than 0.5. From Tab. B.1 the difference for the velocity field is in the order of $10^{-2}$. The pressure field difference for $\omega=100[\mathrm{rpm}]$ is slightly higher, but it can be addressed to the pressure spikes that arise near the inlet, which is a common problem when we consider a constant inlet velocity boundary condition near a wall.

Table B.1 $-L_{2}$ norm of difference for Newtonian Flow.

\begin{tabular}{lcc}
\hline Variable & Rotation $[\mathrm{rpm}]$ & $\|e\|_{L_{2}}$ \\
\hline Velocity Field & 0 & 0.0036 \\
& 100 & 0.0635 \\
Pressure Field & 0 & 0.0201 \\
& 100 & 0.1521 \\
\hline
\end{tabular}

Figure B.2 - Comparison FEniCS minus ANSYS (Newtonian; $\omega=0[\mathrm{rpm}]$ ).

(a)

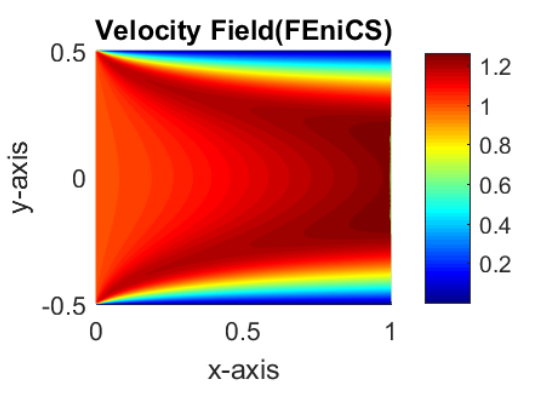

(d)

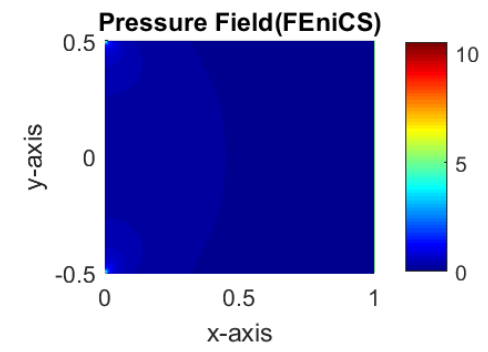

(b)

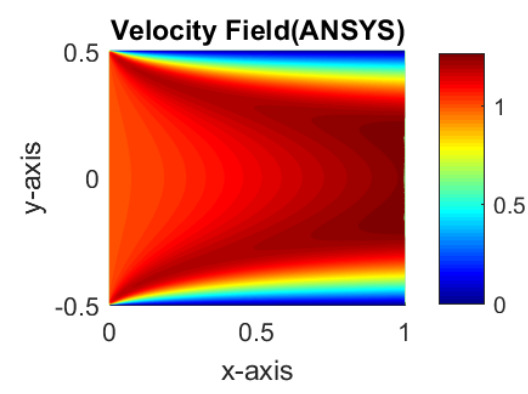

(e)

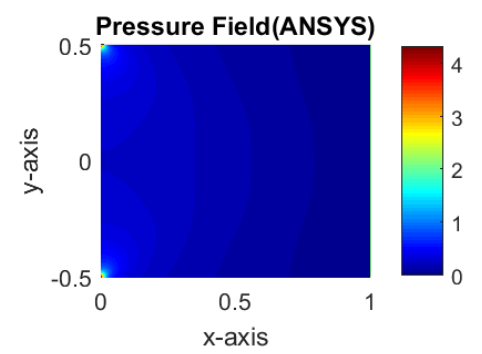

(c)

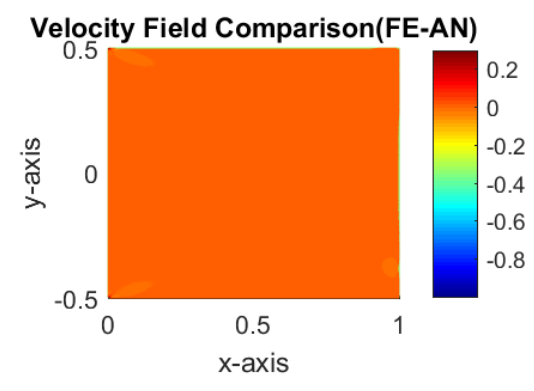

(f)

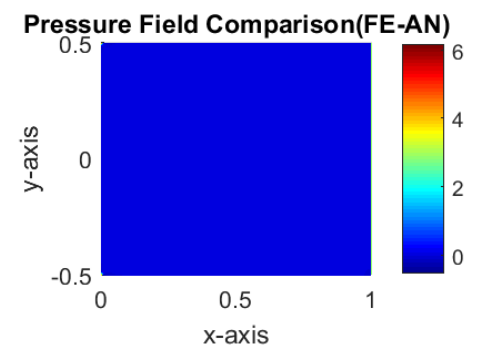


Figure B.3 - Comparison FEniCS minus ANSYS (Newtonian; $\omega=100[\mathrm{rpm}]$ ).

(a)

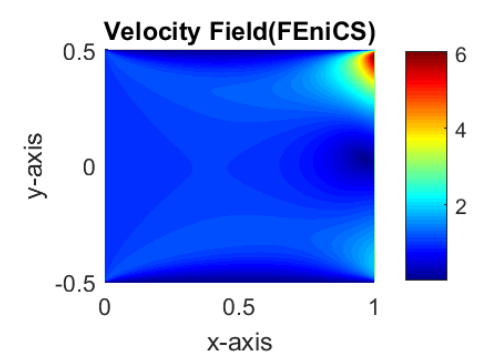

(d)

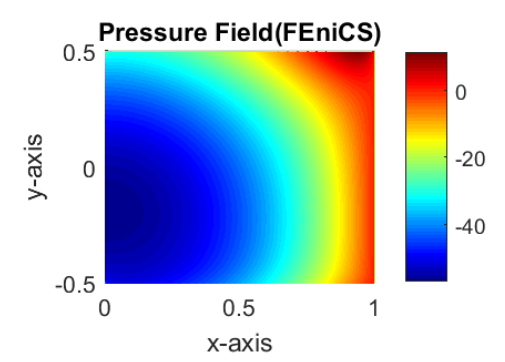

(b)

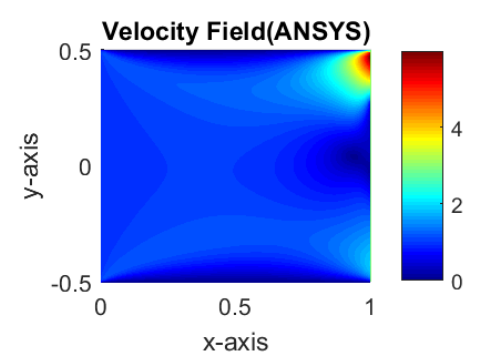

(e)

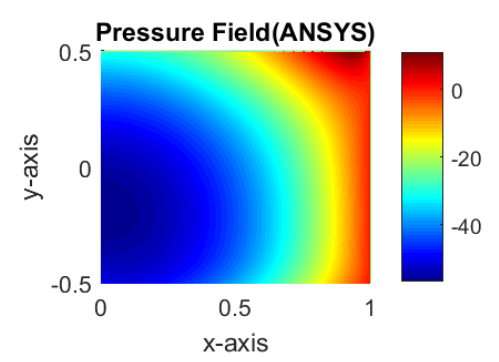

(c)

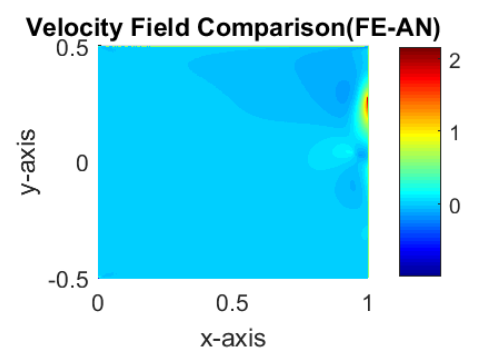

(f)

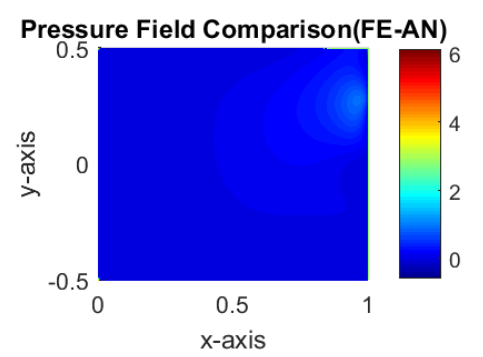

\section{B.2 Non-Newtonian Flow}

The second case considers a laminar non-Newtonian flow in the channel proposed above. Given that the fluid used is blood, all dimensions presented in Fig. B.1 are considered in the CGS system. The variables plots are presented in Figs. B.4 and B.5, for $\omega=0$ and $\omega=100[\mathrm{rpm}]$, respectively. Again, for $\omega=0$ the fields present a very good agreement. The highest differences appear near walls, where the velocity gradients are more intense (Fig. B.4c). The pressure fields are very similar (Figs. B.4d and B.4e). The non-Newtonian viscosity presents the same overall shape (Figs B.4g and B.4h presenting some regions where the FeniCS values are higher than the ones calculated by ANSYS. For $\omega=100$ the peak difference for velocity is more noticeable, but, again, it is present in very few elements. The non-Newtonian viscosity fields, for this rotation, also have very similar shapes, with a small region with high viscosity in the ANSYS result (Fig. B.5h), which may be influenced by the outlet condition implemented in the software.

Table B.2 shows that the difference for the velocity and viscosity fields are in the order of $10^{-2}$, which is very acceptable. The pressure field difference for $\omega=100[\mathrm{rpm}]$ is higher, but again, it can be addressed to the pressure spikes that arise near the inlet as commented in the previous section. 
Table B.2 $-L_{2}$ norm of difference for Newtonian Flow.

\begin{tabular}{lcc}
\hline Variable & Rotation $[\mathrm{rpm}]$ & $\|e\|_{L_{2}}$ \\
\hline Velocity Field & 0 & 0.0424 \\
& 100 & 0.0738 \\
Pressure Field & 0 & 0.1017 \\
& 100 & 2.0889 \\
Non-Newtonian Viscosity Field & 0 & 0.0745 \\
& 100 & 0.0413 \\
\hline
\end{tabular}

Figure B.4 - Comparison FEniCS minus ANSYS (Non-Newtonian; $\omega=0[\mathrm{rpm}]$ ).

(a)

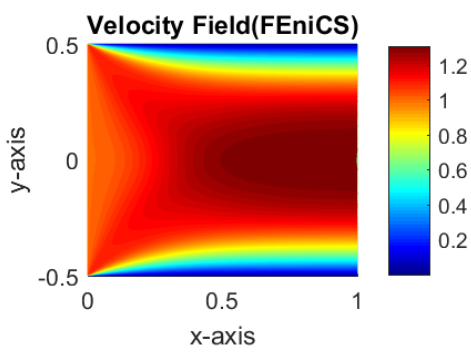

(d)

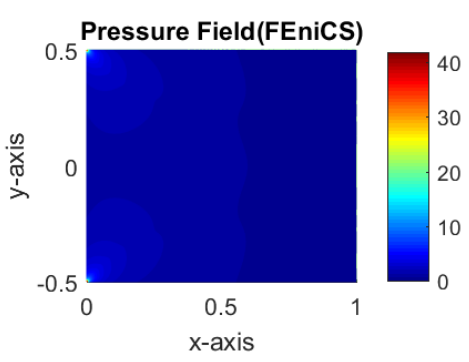

(g)

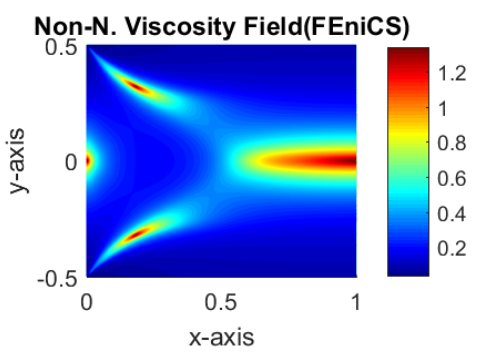

(b)

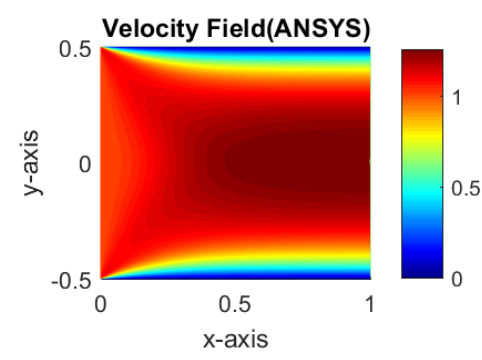

(e)

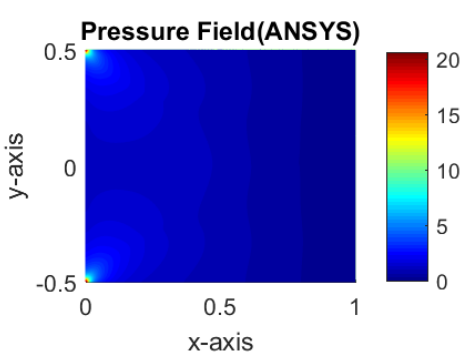

(h)

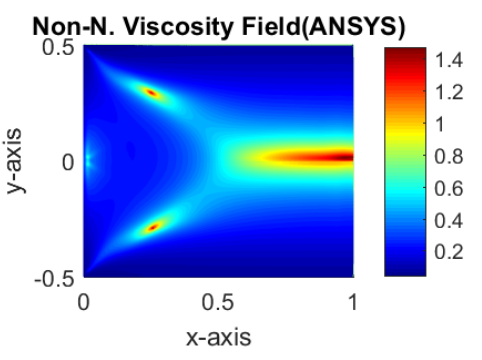

(c)

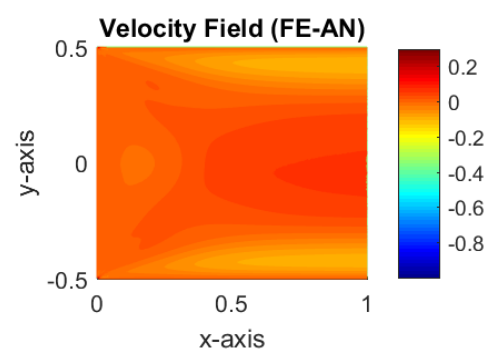

(f)

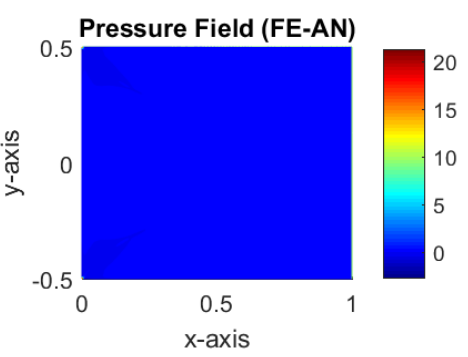

(i)

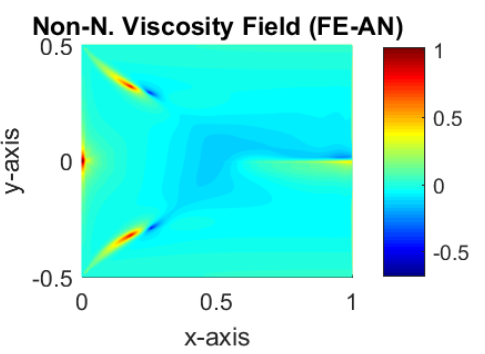


Figure B.5 - Comparison FEniCS minus ANSYS (Non-Newtonian; $\omega=100[\mathrm{rpm}]$ ).

(a)

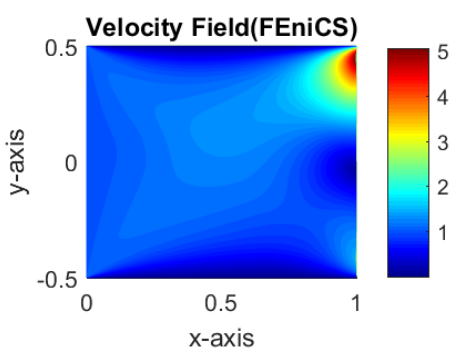

(d)

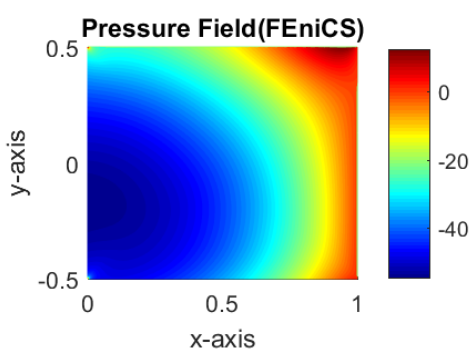

(g)

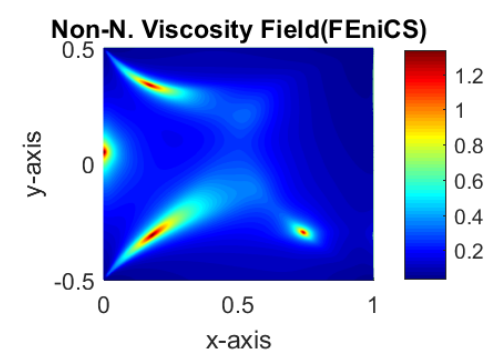

(b)

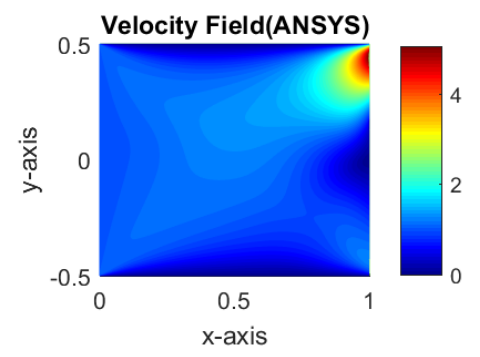

(e)

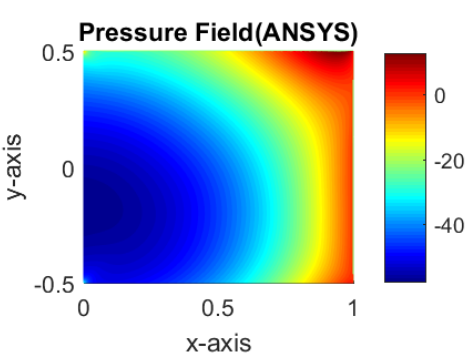

(h)

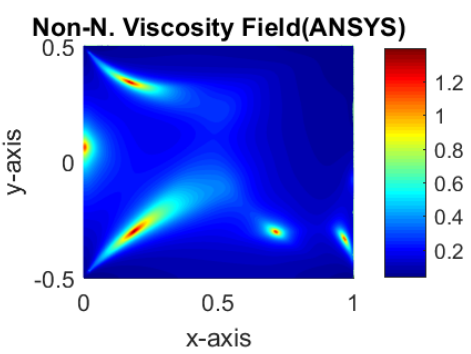

(c)

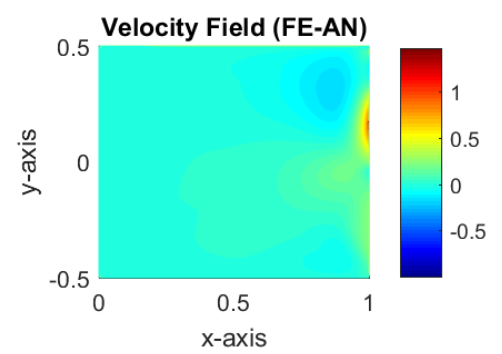

(f)

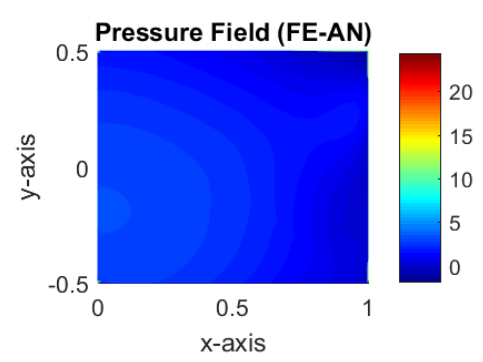

(i)

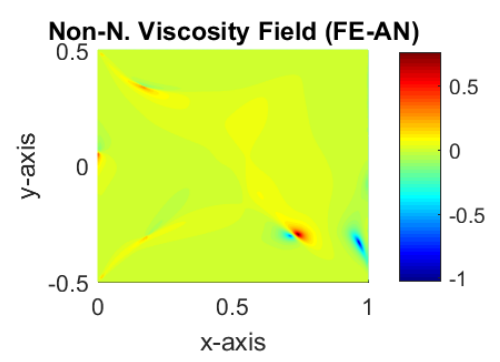

\section{B.3 Turbulent Flow (S-A)}

The third case considers a turbulent Newtonian flow in the channel proposed before. The variables plots are presented in Figs. B.6 and B.7, for $\omega=0$ and $\omega=100[\mathrm{rpm}]$, respectively. The ANSYS CFX implementation of the Spalart-Allmaras model is considered in "beta", i.e., not fully supported. Thus, some of the difference that arises in the results can be addressed to the limitation of the software interface, which does not let the complete removal of wall functions, and to differences in the Spalart-Allmaras equations implemented, given that there are several variations.

For $\omega=0$ the fields present a very good agreement. The highest differences appear near the inlet corner (Fig. B.6c), and it may be addressed to wall functions implemented by ANSYS. The pressure fields are very similar (Figs. B.6d and B.6e). The turbulent viscosity fields ( $\nu_{t}$ of Eq. 3.13) present the same overall shape (Figs B.6g and B.6h), with most of the differences occurring near the inlet, which influences the diffusion terms. The ANSYS implementation seems to adjust the boundary condition arbitrarily, given that 
constant inlet viscosity is changed abruptly in this region (Fig. B.6h), while the FEniCS distribution shows a more smooth diffusion (Fig. B.6g).

For $\omega=100$, the difference in velocities is more noticeable with a mean magnitude difference of 0.5 , which is not too high in this case. The turbulent viscosity fields for this rotation have very similar shapes, with the highest differences occurring near the inlet. Even though the fields present some divergences, Tab. B.2 shows that the difference for the velocity and viscosity fields are of the second order, which is very acceptable.

Figure B.6 - Comparison FEniCS minus ANSYS (Turbulent Newtonian; $\omega=0[\mathrm{rpm}]$ ).

(a)

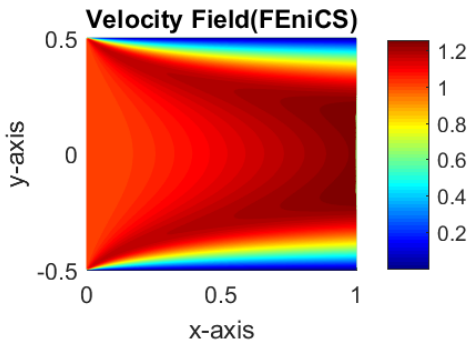

(d)

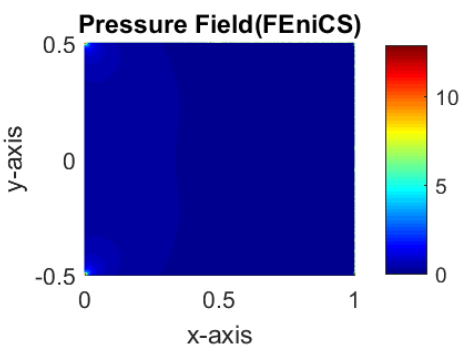

(g)

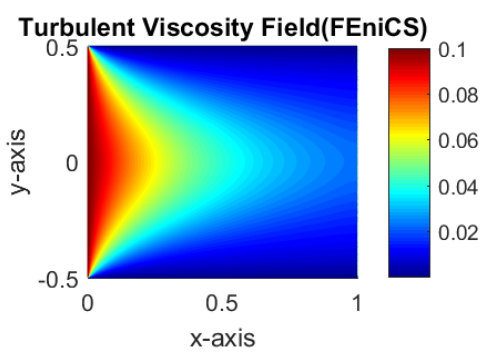

(b)

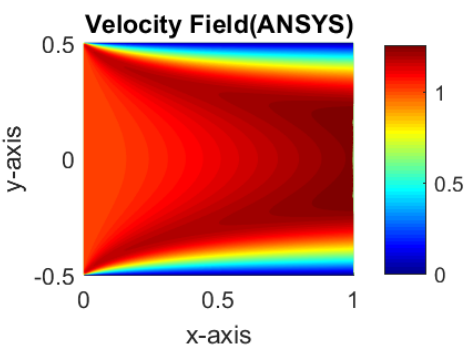

(e)

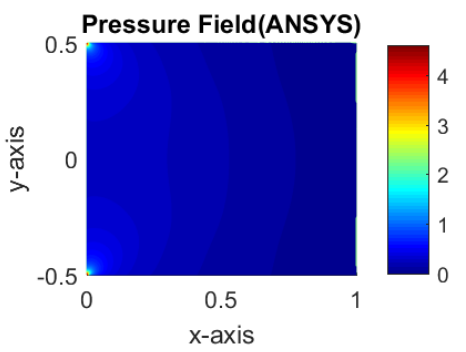

(h)

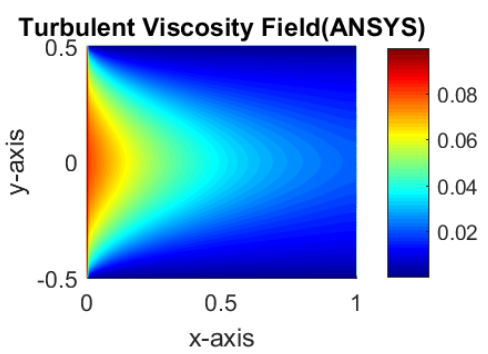

(c)

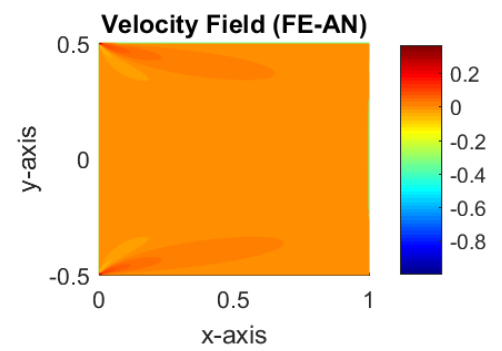

(f)

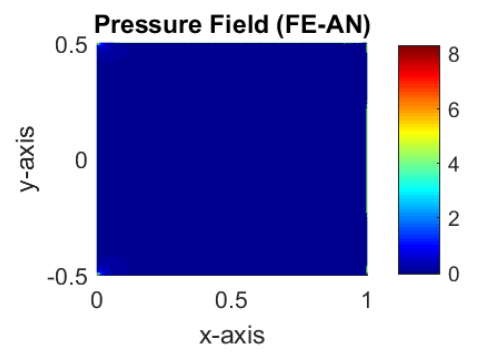

(i)

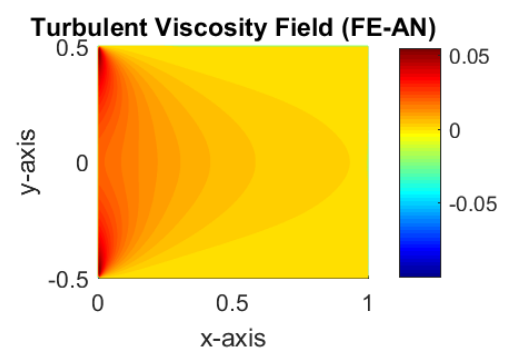


Table B.3 $-L_{2}$ norm of difference for Turbulent Flow (FEniCS - ANSYS).

\begin{tabular}{lcc}
\hline Variable & Rotation $[\mathrm{rpm}]$ & $\|e\|_{L_{2}}$ \\
\hline Velocity Field & 0 & 0.0090 \\
& 100 & 0.0579 \\
Pressure Field & 0 & 0.0545 \\
& 100 & 0.3002 \\
Turbulent Viscosity $\left(\nu_{t}\right)$ & 0 & 0.0085 \\
& 100 & 0.0103 \\
\hline
\end{tabular}

Figure B.7 - Comparison FEniCS minus ANSYS (Turbulent Newtonian; $\omega=100[\mathrm{rpm}]$ ).

(a)

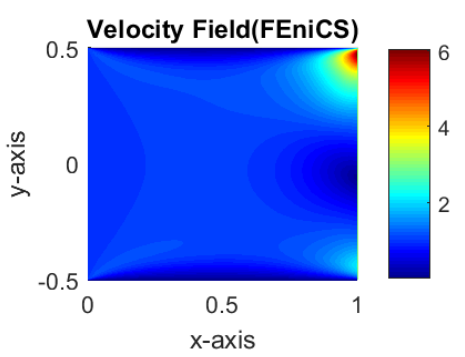

(d)

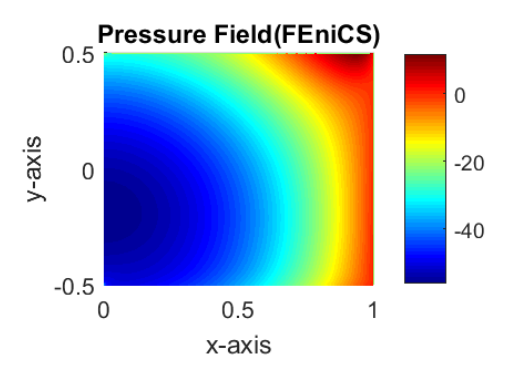

(g)

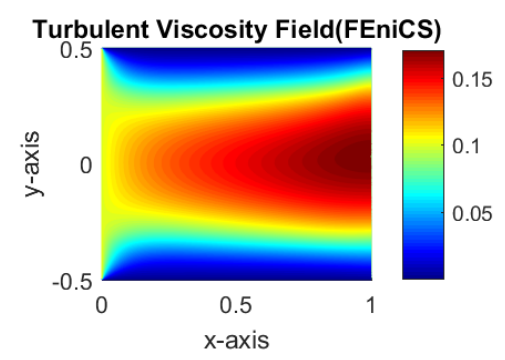

(b)

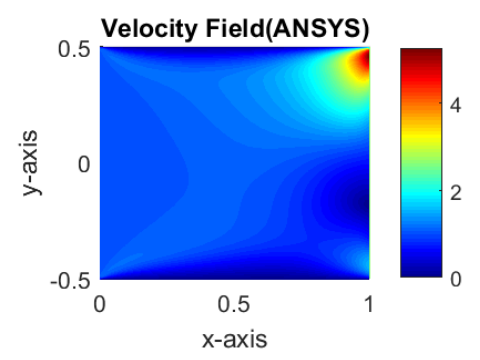

(e)

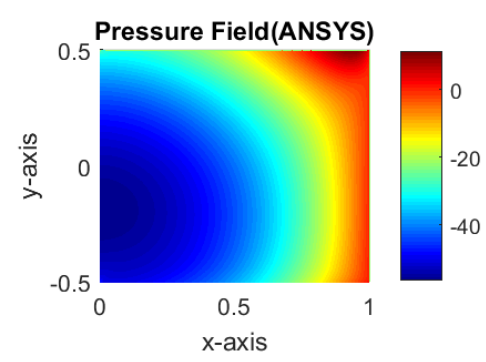

(h)

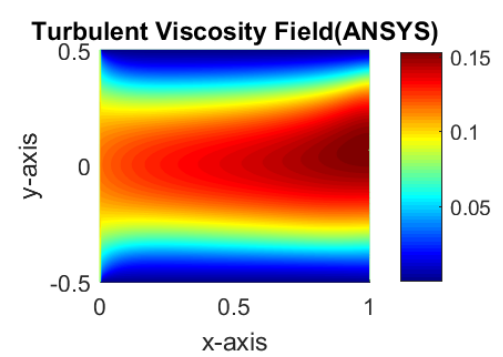

(c)

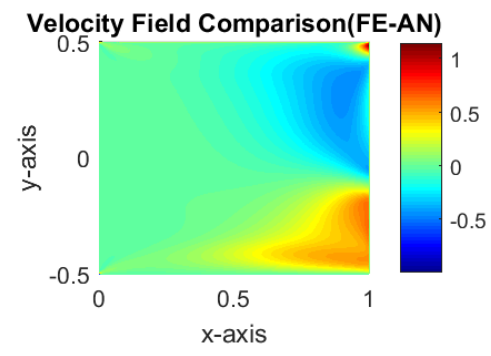

(f)

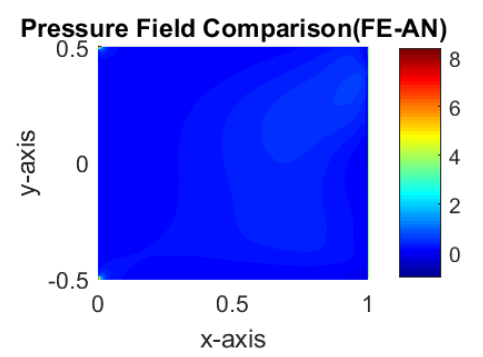

(i)

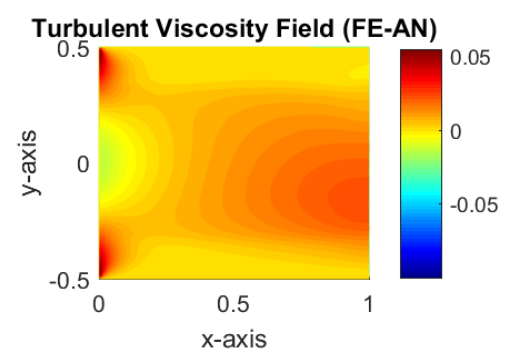

In order to perform further verification, the same model is implemented in OpenFOAM, and the results are shown in Fig. B.8. The turbulent viscosity fields are now much closer, with the difference in magnitude near zero. However, the other fields (velocity and pressure) present a higher divergence. From Tab. B.4 we can see that the difference for viscosity is in the order of $10^{-4}$, while the others are of first order. These divergences may appear due to some differences in the problem discretization, solver method, boundary conditions, 
and more, as shown in Tab. B.4

Figure B.8 - Comparison FEniCS minus OpenFOAM (Turbulent Newtonian; $\omega=100[$ rpm $]$ ).

(a)

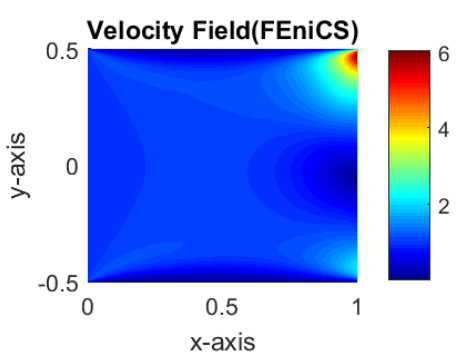

(d)

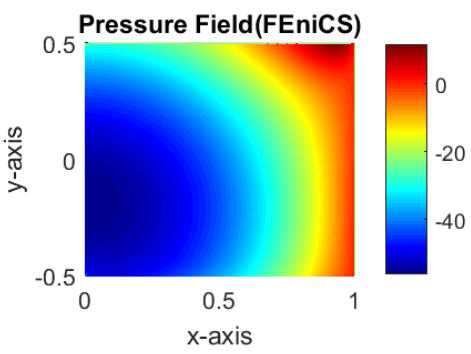

(g)

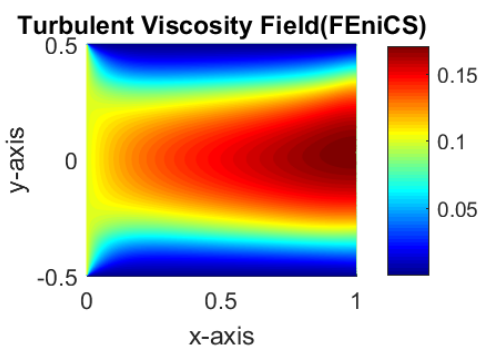

(b)

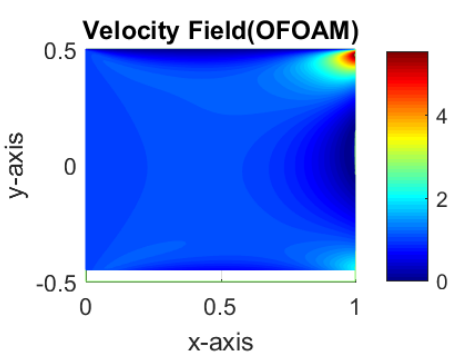

(e)

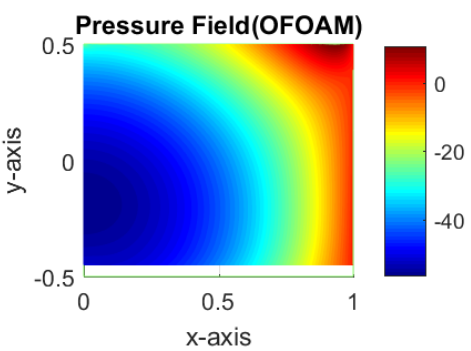

(h)

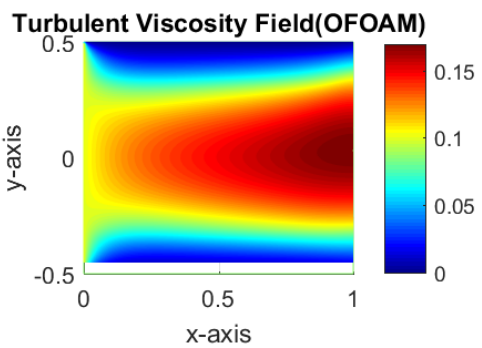

(c)

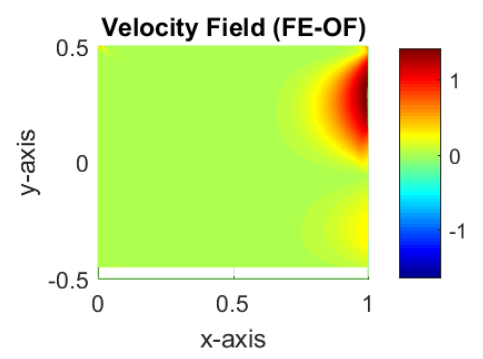

(f)

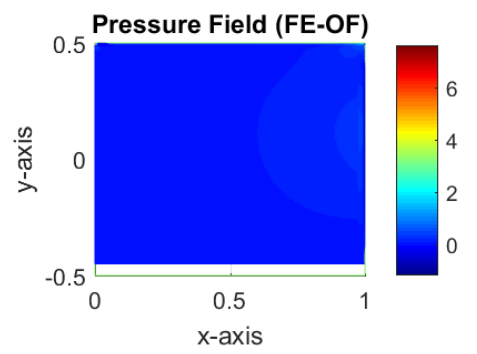

(i)

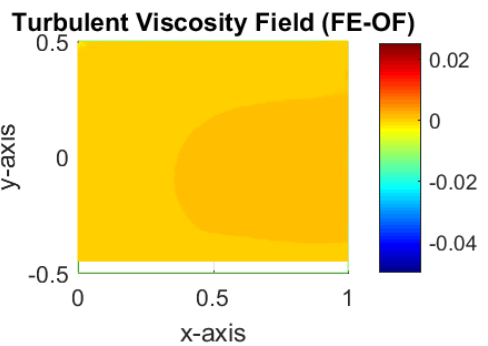

Table B.4 - $L_{2}$ norm of difference for Turbulent Flow (FEniCS - OpenFOAM).

\begin{tabular}{lcc}
\hline Variable & Rotation $[\mathrm{rpm}]$ & $\|e\|_{L_{2}}$ \\
\hline Velocity Field & 100 & 0.2073 \\
Pressure Field & 100 & 0.1382 \\
Turbulent Viscosity $\left(\nu_{t}\right)$ & 100 & 0.0006 \\
\hline
\end{tabular}


Table B.5 - Problem Implementation Comparison.

\begin{tabular}{lccc}
\hline & FEniCS & ANSYS CFX & OpenFOAM \\
\hline Discretization & Finite Element & Finite Volume & Finite Volume \\
Solver Method & Direct Newton Solver & SIMPLE & SIMPLE \\
Outlet Condition & Zero Stress Gradient & Zero Pressure & Zero Pressure \\
Wall Function & Not Used & Not Clear & Not Used \\
S-A model Tested & Baseline & Not Clear & Baseline \\
Element Interpolation & Q2Q1 & Q1Q1 & Q1Q1 \\
Domain Space & 2D & 3D with symmetry & 2D \\
\hline
\end{tabular}




\section{APPENDIX C - SENSITIVITY VERIFICATION BY FINITE DIFFERENCE METHOD}

In this appendix, we perform the sensitivity verification by comparing the sensitivity obtained via the Adjoint Method with the one obtained via the Finite Difference Method. Given that the later have a high computational cost, the sensitivity is verified only on a few trial points. Also, as we use the automatic derivation each flow case (Newtonian, non-Newtonian, and turbulent) need to be verified individually. This is done in the following sections. Figure C.1 shows the domain and trial points positions used in all cases. The mesh used has 57,600 elements; the boundary conditions are the same as in Sec. 8.3. the material distribution used is homogeneous with $\alpha=0.3$ (design variable), and the functional analysed is the energy dissipation.

Figure C.1 - Trial points for sensitivity verification.

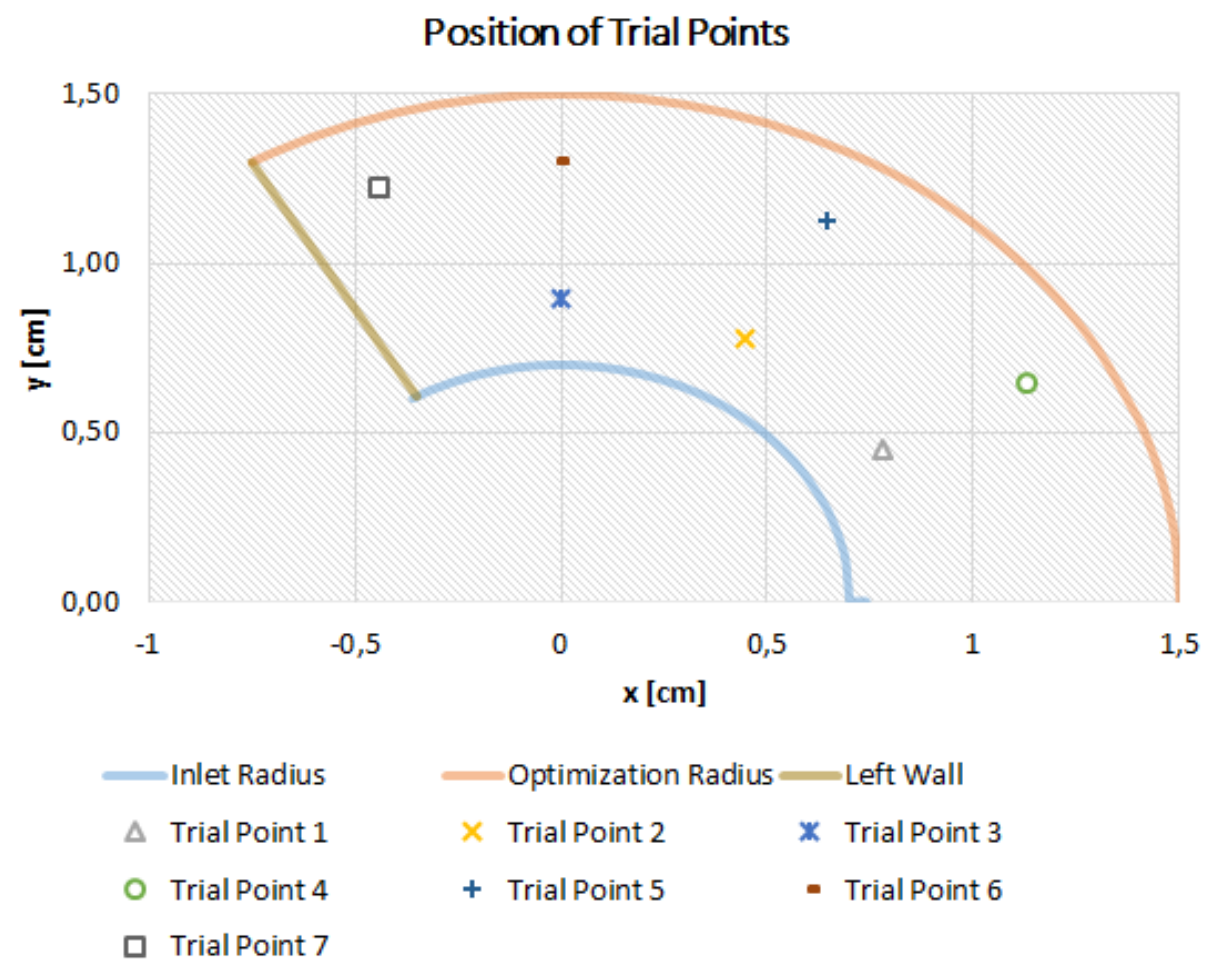




\section{C.1 Finite Differences}

The sensitivity calculation via finite differences can be achieved in different ways, in this case, we use the Central Differences, and it is given by:

$$
\frac{\partial J}{\partial \alpha}=\frac{J(\alpha+h)-J(\alpha-h)}{2 h}
$$

where $J$ is the desired functional, $\alpha$ is the design variable and $h$ is the perturbation size. In all cases the tested functional is the energy dissipation.

\section{C.2 Newtonian Flow}

In this section, the sensitivity is verified for the rotating Newtonian flow, which considers constant viscosity in the formulation. Table C.1 shows the computed sensitivities at the trial points and the difference between the finite difference and adjoint values. The perturbation value must be sufficiently low to get the correct approximation, but high enough to avoid the machine precision. Hence we use a perturbation of $h=1 \cdot 10^{-6}$. As we can see, the values are very close, and the highest differences $(-2.86 \%)$ occurs at points with low sensitivity values (which is expected). Thus, the automatic derivation performed with pyadjoint is acceptable.

Table C.1 - Sensitivity Verification for Newtonian Flow $\left(h=1 \cdot 10^{-6}\right)$.

\begin{tabular}{ccccccc}
\hline Trial Point & Radius & $\mathrm{X}$ & $\mathrm{Y}$ & Central Diff. & Adjoint & Difference $\%$ \\
\hline 1 & 0.9 & 0.78 & 0.45 & -0.0491 & -0.0493 & $0.40 \%$ \\
2 & 0.9 & 0.45 & 0.78 & -0.3685 & -0.3682 & $-0.07 \%$ \\
3 & 0.9 & 0.00 & 0.90 & -0.0178 & -0.0179 & $0.42 \%$ \\
4 & 1.3 & 1.13 & 0.65 & -0.0051 & -0.0050 & $-2.86 \%$ \\
5 & 1.3 & 0.65 & 1.13 & -0.0314 & -0.0313 & $-0.26 \%$ \\
6 & 1.3 & 0.00 & 1.30 & -0.2318 & -0.2317 & $-0.02 \%$ \\
7 & 1.3 & -0.44 & 1.22 & -0.1012 & -0.1014 & $0.23 \%$ \\
\hline
\end{tabular}

\section{C.3 Non-Newtonian Flow}

In this section, the sensitivity is verified for the rotating non-Newtonian flow, which considers the Carreu-Yasuda model to calculate the viscosity. Table C.2 shows the computed sensitivities at the trial points and the difference between the finite difference and adjoint values. Again, the values are very close, and the highest difference is very low (1.5\%). Also, when the values of Tab. C.1 and C.2 are compared we notice that the values are slightly different indicating that the non-Newtonian model does have an influence on the overall sensitivity. 
Table C.2 - Sensitivity Verification for Non-Newtonian Flow $\left(h=1 \cdot 10^{-6}\right)$.

\begin{tabular}{ccccccc}
\hline Trial Point & Radius & $\mathrm{X}$ & $\mathrm{Y}$ & Central Diff. & Adjoint & Difference $\%$ \\
\hline 1 & 0.9 & 0.78 & 0.45 & -0.0490 & -0.0491 & $0.22 \%$ \\
2 & 0.9 & 0.45 & 0.78 & -0.3673 & -0.3675 & $0.03 \%$ \\
3 & 0.9 & 0.00 & 0.90 & -0.0175 & -0.0177 & $1.50 \%$ \\
4 & 1.3 & 1.13 & 0.65 & -0.0051 & -0.0051 & $-1.24 \%$ \\
5 & 1.3 & 0.65 & 1.13 & -0.0306 & -0.0306 & $0.07 \%$ \\
6 & 1.3 & 0.00 & 1.30 & -0.2308 & -0.2306 & $-0.09 \%$ \\
7 & 1.3 & -0.44 & 1.22 & -0.1026 & -0.1025 & $-0.09 \%$ \\
\hline
\end{tabular}

\section{C.4 Turbulent Flow}

In this section, the sensitivity is verified for the rotating turbulent flow, which considers the Spalart-Allmaras model to calculate the turbulent viscosity. Table C.3 shows the computed sensitivities at the trial points and the difference between the finite difference and adjoint values. The difference is very small between the adjoint and the central difference sensitivity values, and the highest value occurs at a point with very small sensitivity. Hence, the adjoint is operating correctly.

Table C.3 - Sensitivity Verification for Turbulent Flow $\left(h=1 \cdot 10^{-6}\right)$.

\begin{tabular}{ccccccc}
\hline Trial Point & Radius & $\mathrm{X}$ & $\mathrm{Y}$ & Central Diff. & Adjoint & Difference \% \\
\hline 1 & 0.9 & 0.78 & 0.45 & -0.1432 & -0.1433 & $0.09 \%$ \\
2 & 0.9 & 0.45 & 0.78 & -1.3269 & -1.3270 & $0.00 \%$ \\
3 & 0.9 & 0.00 & 0.90 & -1.1017 & -1.1018 & $0.01 \%$ \\
4 & 1.3 & 1.13 & 0.65 & -0.0007 & -0.0007 & $2.63 \%$ \\
5 & 1.3 & 0.65 & 1.13 & -0.0602 & -0.0602 & $-0.05 \%$ \\
6 & 1.3 & 0.00 & 1.30 & -0.2537 & -0.2537 & $0.00 \%$ \\
7 & 1.3 & -0.44 & 1.22 & -0.3078 & -0.3078 & $0.01 \%$ \\
\hline
\end{tabular}




\section{APPENDIX D - MESH CONVERGENCE ANALYSIS}

In this appendix, the mesh convergence analysis is performed for the simulation of the complete pump model (impeller and volute) performed with ANSYS. Two mesh aspects need to be analyzed, first the $y+$ near the walls, due to the use of turbulence models, and the variation of the result with the change in the mesh discretization (element size). All the results presented here consider a mass flow of $3[L / \mathrm{min}]$ and a rotation of $3000[\mathrm{rpm}]$.

\section{D.1 $Y+$ Analysis}

The turbulence model used is the $k-\omega S S T$; thus, the literature indicates that near the wall, the condition is $y+\approx 1$. The control of the $y+$ plus value is done by refining the first layer of elements near walls and using the mesh inflation to adequate the increase in the element size until the region outside the boundary layer is reached. In this case, the first layer with $2 \cdot 10^{-3}[\mathrm{~mm}]$ element size is used coupled with inflation that has 15 layers and 1.2 growth rate. The $y+$ distribution for the finest mesh of the Straight Blade case is shown in Fig. D.1, the average value for this mesh is 0.39 . The distribution for the Optimized Blade case is shown in Fig. D.2 and the average value is 0.34 .

Figure D.1 $-Y+$ distribution for the Straight Blade Case.

(a) Impeller.

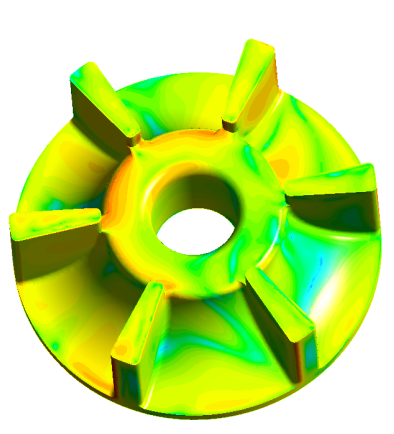

(b) Volute.

Yplus
$1.5 \mathrm{e}+00$
$1.1 \mathrm{e}+00$
$8.2 \mathrm{e}-01$
$6.1 \mathrm{e}-01$
$4.6 \mathrm{e}-01$
$3.4 \mathrm{e}-01$
$2.5 \mathrm{e}-01$
$1.9 \mathrm{e}-01$
$1.4 \mathrm{e}-01$
$1.1 \mathrm{e}-01$
$7.9 \mathrm{e}-02$
$5.9 \mathrm{e}-02$
$4.4 \mathrm{e}-02$
$3.3 \mathrm{e}-02$
$2.5 \mathrm{e}-02$

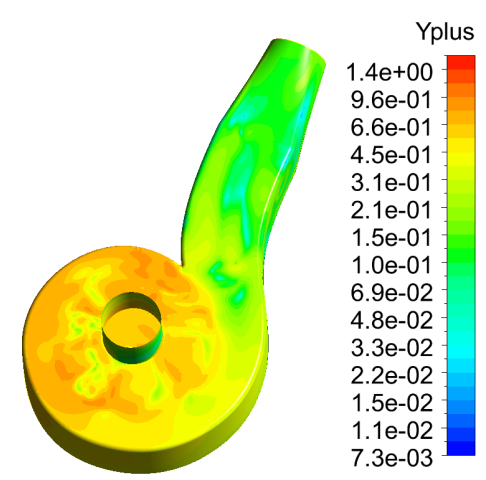


Figure D.2 $-Y+$ distribution for the Optimized Blade Case.

(a) Impeller.

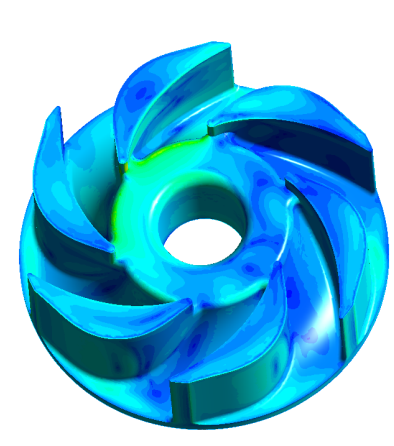

(b) Volute.

Yplus
$1.7 e+00$
$1.6 e+00$
$1.5 e+00$
$1.4 e+00$
$1.2 e+00$
$1.1 e+00$
$9.9 e-01$
$8.7 e-01$
$7.4 e-01$
$6.2 e-01$
$5.0 e-01$
$3.7 e-01$
$2.5 e-01$
$1.2 e-01$
$0.0 e+00$

\section{D.2 Mesh Refinement Analysis}

The other aspect analyzed is the change in the simulation results as the mesh is refined. It is important to notice that for all changes in the element size, the condition $y+\approx 1$ (at walls) still needs to be respected. The mesh convergence analysis needs to be verified for all quantities of interest. In this case, not only the pressure but also the energy dissipation and vorticity must be verified. The minimum element size that we are able to reach with the current computational power is $0.2[\mathrm{~mm}]$, which gives around 43 million elements.

Figure D.3 shows the mesh convergence comparison for the newtonian case (water). The pressure curve (Fig. D.3a) shows that there is a low variation of the pressure value with the mesh refinement. The energy dissipation curve (Fig. D.3b shows that the optimized case stabilizes with relatively a low number of elements, while the straight blade case tends to increase the value. This can be explained by the fact that with smaller elements other phenomena of small scales start to appear in the model, such as small scale vortex. The same behavior is observed for the vorticity functional (Fig. D.3c), given that it is partially proportional to the velocity gradient.

Table D.1 shows the mesh functional convergence for the Straight Blade case for the newtonian case (water). As we can see after the first refinements, the difference between steps increases a little as the number of elements increase. This shows that as the mesh refinement increases, phenomena of a smaller scale are captured by the simulation, such as small re-circulations. This increases the calculated velocity gradient and so the energy dissipation and the vorticty. Table D.2 shows the same analysis for the Optimized Blade case. All functional values have very small variations (lower than $0.5 \%$ ). Thus, this most refined mesh is used in all the simulations presented in this work. 
Figure D.3 - Mesh Convergence Curves for Water.

(a)

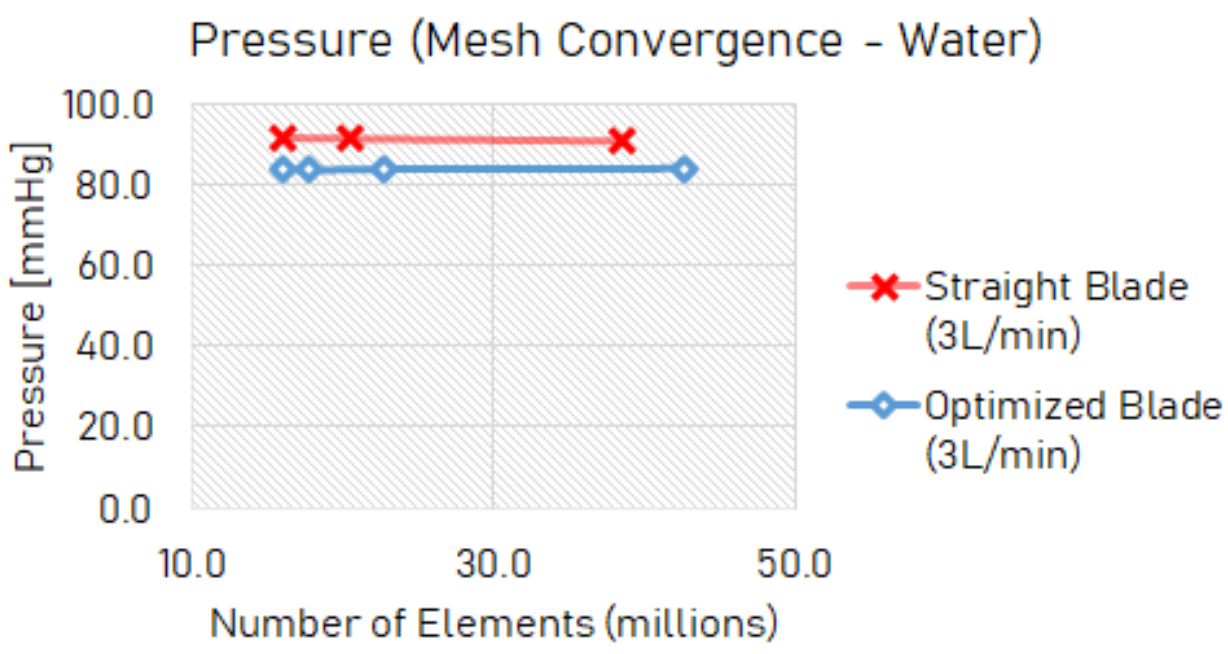

(b)

Energy Dissipation (Mesh Conv. - Water)

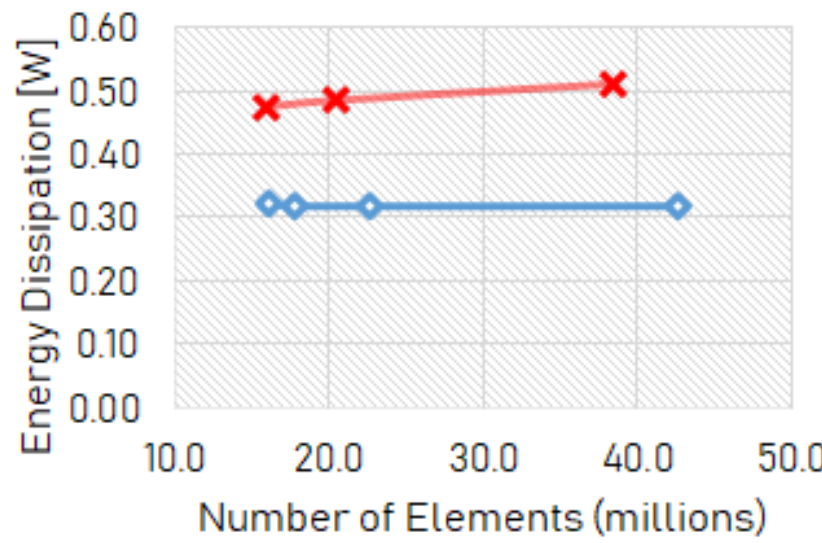

$\mathbf{X}$-Straight Blade (3L/min)

$\multimap 0$ Optimized Blade (3L/min)

(c)

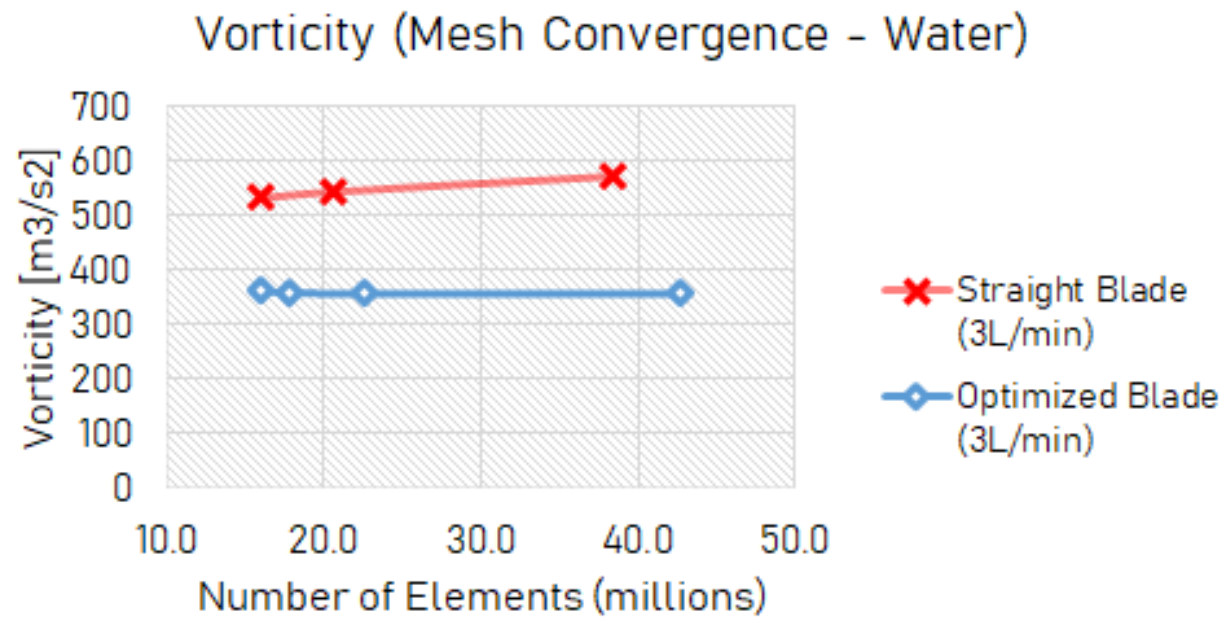


Table D.1 - Mesh Convergence Data for the Straight Blade Case with Water.

\begin{tabular}{ccccccccc}
\hline $\begin{array}{c}\text { Element } \\
\begin{array}{c}\text { Size } \\
{[\mathrm{mm}]}\end{array}\end{array}$ & $\begin{array}{c}\text { Num. } \\
\text { Elements } \\
{[\text { millions }]}\end{array}$ & $\begin{array}{c}\text { Pressure } \\
{[\mathrm{mmHg}]}\end{array}$ & $\%$ & $\begin{array}{c}\text { Energy } \\
\text { Dissipation } \\
{[W]}\end{array}$ & $\%$ & $\begin{array}{c}\text { Vorticity } \\
{\left[\mathrm{m}^{2} / \mathrm{s}^{2}\right]}\end{array}$ & $\%$ & $y+$ \\
\hline 0.4 & 16.0 & 91.5 & - & 0.48 & - & 533.2 & - & 0.82 \\
0.3 & 20.5 & 91.3 & $-0.3 \%$ & 0.49 & $2.1 \%$ & 544.5 & $2.1 \%$ & 0.82 \\
0.2 & 38.5 & 90.7 & $-0.6 \%$ & 0.51 & $5.3 \%$ & 573.3 & $5.3 \%$ & 0.85 \\
\hline
\end{tabular}

Table D.2 - Mesh Convergence Data for the Optimized Blade Case with Water.

\begin{tabular}{ccccccccc}
\hline $\begin{array}{c}\text { Element } \\
\text { Size } \\
{[\mathrm{mm}]}\end{array}$ & $\begin{array}{c}\text { Num. } \\
\text { Elements } \\
{[\text { millions }]}\end{array}$ & $\begin{array}{c}\text { Pressure } \\
{[\mathrm{mmHg}]}\end{array}$ & $\%$ & $\begin{array}{c}\text { Energy } \\
\text { Dissipation } \\
{[\mathrm{W}]}\end{array}$ & $\%$ & $\begin{array}{c}\text { Vorticity } \\
{\left[\mathrm{m}^{2} / \mathrm{s}^{2}\right]}\end{array}$ & $\%$ & $y+$ \\
\hline 0.5 & 16.0 & 83.9 & - & 0.32 & - & 362.1 & - & 0.51 \\
0.4 & 17.7 & 83.5 & $-0.5 \%$ & 0.32 & $-0.8 \%$ & 359.0 & $-0.9 \%$ & 0.51 \\
0.3 & 22.6 & 83.7 & $0.3 \%$ & 0.32 & $-0.4 \%$ & 357.1 & $-0.5 \%$ & 0.50 \\
0.2 & 42.7 & 83.9 & $0.2 \%$ & 0.32 & $0.2 \%$ & 357.7 & $0.2 \%$ & 0.50 \\
\hline
\end{tabular}

The same analysis is performed for both VADs with blood (non-Newtonian) at 3 $[L / \mathrm{min}]$ and $3000[\mathrm{rpm}]$. Figure D.4 shows the mesh convergence curves for both cases. The use of the non-Newtonian fluid causes a faster mesh convergence (with less elements). The curves stabilize around 30 million of elements for both VADs. Table D.3 shows the convergence values for the straight-bladed case and Tab. D.4 for the optimized case. The most refined meshes (around 40 million elements) are used in the simulations presented in the results chapter. These meshes are used for the simulations at $5[\mathrm{~L} / \mathrm{min}]$ as well. 
Figure D.4 - Mesh Convergence Curves for Blood.

(a)

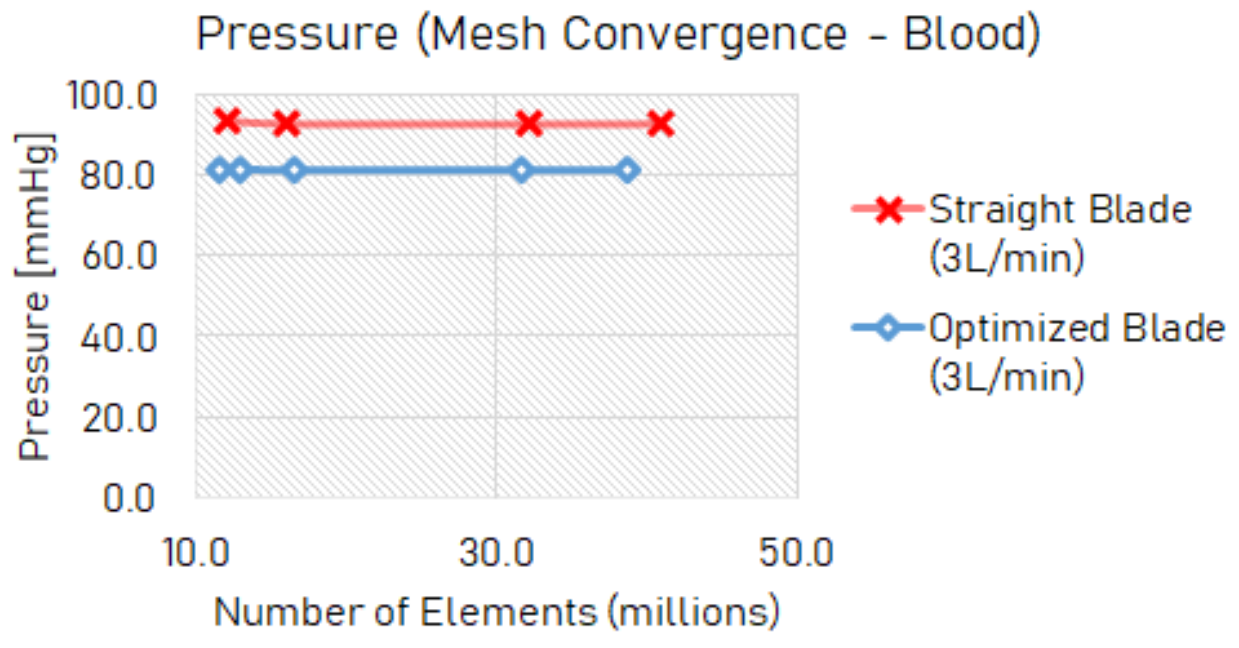

(b)

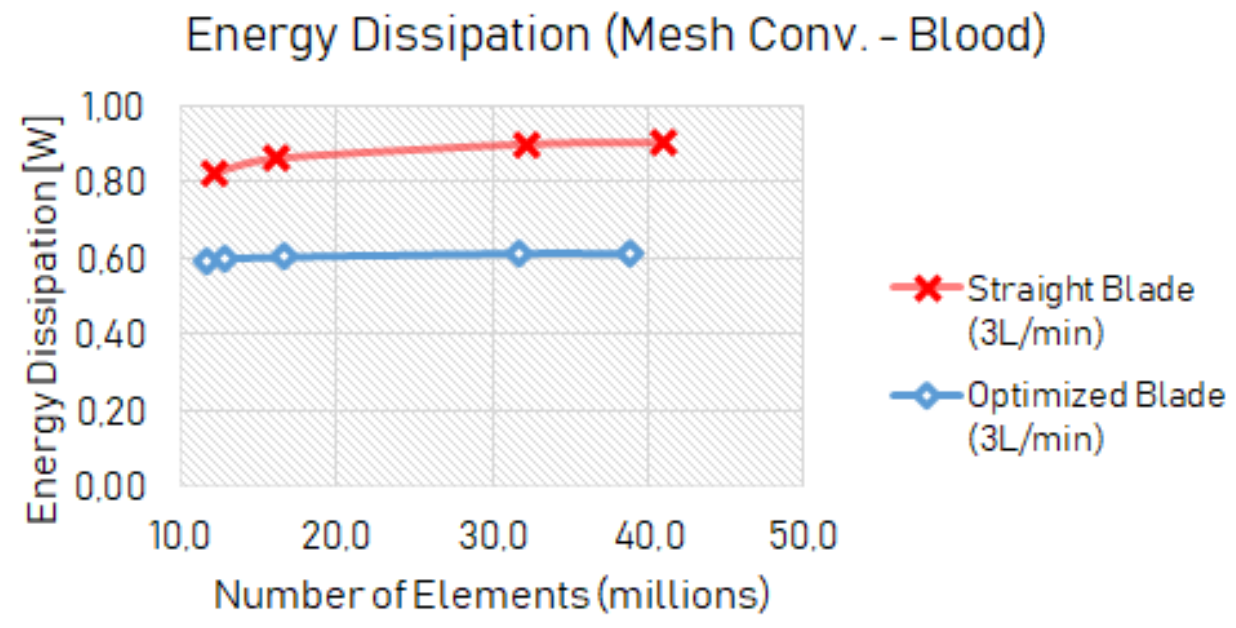

(c)

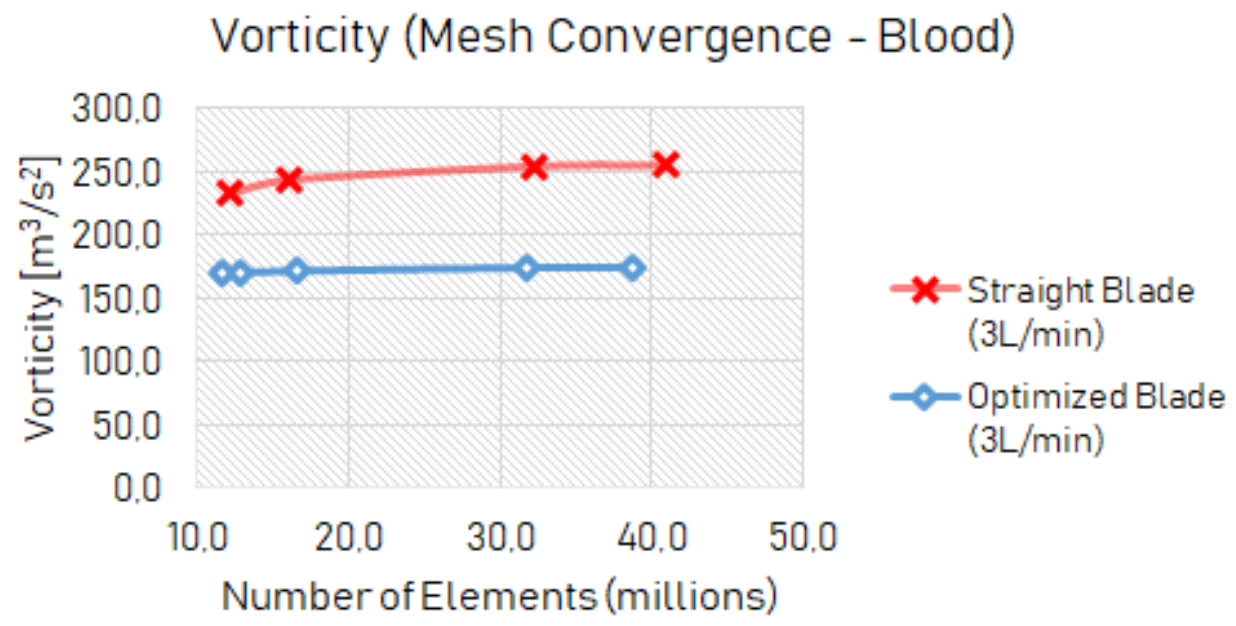


Table D.3 - Mesh Convergence Data for the Straight Blade Case with Blood.

\begin{tabular}{ccccccccc}
\hline $\begin{array}{c}\text { Element } \\
\text { Size } \\
{[\mathrm{mm}]}\end{array}$ & $\begin{array}{c}\text { Num. } \\
\text { Elements } \\
{[\text { millions }]}\end{array}$ & $\begin{array}{c}\text { Pressure } \\
{[\mathrm{mmHg}]}\end{array}$ & $\%$ & $\begin{array}{c}\text { Energy } \\
\text { Dissipation } \\
{[W]}\end{array}$ & $\%$ & $\begin{array}{c}\text { Vorticity } \\
{\left[\mathrm{m}^{2} / \mathrm{s}^{2}\right]}\end{array}$ & $\%$ & $y+$ \\
\hline 0.4 & 12.2 & 93.5 & & 0.82 & & 232.4 & & 0.79 \\
0.3 & 16.1 & 92.7 & $-0.8 \%$ & 0.86 & $4.6 \%$ & 243.2 & $4.7 \%$ & 0.81 \\
0.2 & 32.2 & 92.6 & $-0.1 \%$ & 0.90 & $4.3 \%$ & 253.7 & $4.3 \%$ & 0.83 \\
0.18 & 41.0 & 92.8 & $0.2 \%$ & 0.90 & $0.4 \%$ & 254.8 & $0.4 \%$ & 0.84 \\
\hline
\end{tabular}

Table D.4 - Mesh Convergence Data for the Optimized Blade Case with Blood.

\begin{tabular}{ccccccccc}
\hline $\begin{array}{c}\text { Element } \\
\text { Size } \\
{[\mathrm{mm}]}\end{array}$ & $\begin{array}{c}\text { Num. } \\
\text { Elements } \\
{[\text { millions }]}\end{array}$ & $\begin{array}{c}\text { Pressure } \\
{[\mathrm{mmHg}]}\end{array}$ & $\%$ & $\begin{array}{c}\text { Energy } \\
\text { Dissipation } \\
{[W]}\end{array}$ & $\%$ & $\begin{array}{c}\text { Vorticity } \\
{\left[\mathrm{m}^{2} / \mathrm{s}^{2}\right]}\end{array}$ & $\%$ & $y+$ \\
\hline 0.5 & 16.0 & 83.9 & & 0.32 & & 362.1 & & 0.51 \\
0.4 & 17.7 & 83.5 & $-0.5 \%$ & 0.32 & $-0.8 \%$ & 359.0 & $-0.9 \%$ & 0.51 \\
0.3 & 22.6 & 83.7 & $0.3 \%$ & 0.32 & $-0.4 \%$ & 357.1 & $-0.5 \%$ & 0.50 \\
0.2 & 42.7 & 83.9 & $0.2 \%$ & 0.32 & $0.2 \%$ & 357.7 & $0.2 \%$ & 0.50 \\
\hline
\end{tabular}

